\title{
Giant Dipole Resonance Parameters of Ground-State Photoabsorption: Experimental Values with UnCertainties
}

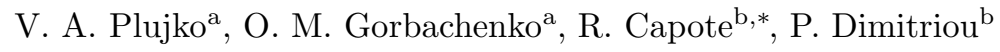 \\ ${ }^{a}$ Nuclear Physics Department, Taras Shevchenko National University, Kyiv, Ukraine \\ ${ }^{b}$ NAPC-Nuclear Data Section, International Atomic Energy Agency, Vienna, Austria
}

\begin{abstract}
Updated values and corresponding uncertainties of Isovector Giant Dipole Resonance (GDR) parameters which are obtained by the least-squares fitting of theoretical photoabsorption cross sections to experimental data are presented. The theoretical photoabsorption cross sections are taken as a sum of the components corresponding to the excitation of the GDR and quasideuteron photodisintegration. The current compilation is an extension and improvement of the earlier compilations of Lorentzian parameters for ground-state photoneutron and photoabsorption cross sections and covers experimental data made available up to June 2017.
\end{abstract}

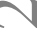

*Corresponding author.

Email address: R.CapoteNoy@iaea.org (R. Capote) 


\section{Contents}

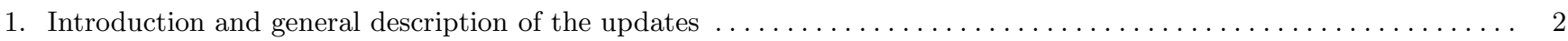

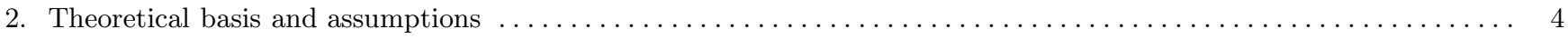

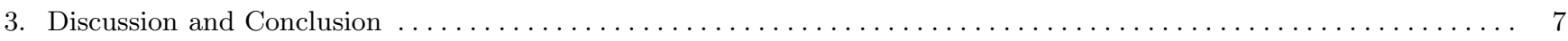

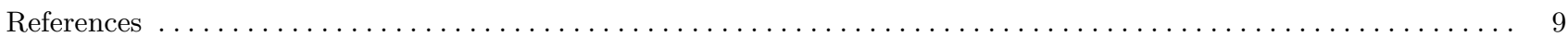

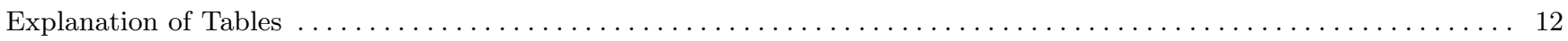

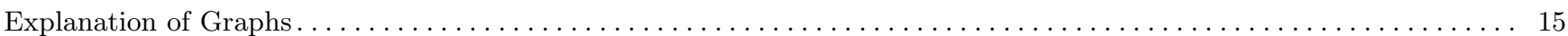

Tables

1. Experimental values and uncertainties of GDR parameters within Standard Lorentzian (SLO) approach. .... . 21

2. Experimental values and uncertainties of GDR parameters within Modified Lorentzian (SMLO) approach.... . 29

3. References to experimental and evaluated cross section data taken from EXFOR. . . . . . . . . . . . . 37

Graphs $1-162$

\section{Introduction and general description of the updates}

Isovector Giant Dipole Resonances (GDR) are fundamental modes of nuclear collective excitations beyond the binding energy per nucleon. Nuclear collective states correspond to vibrations of the nucleons and are strongly manifested in electric dipole (E1) gamma-transitions which dominate over transitions of other monopole order when they occur simultaneously (see, for example, [1-3] for references). This allows us to obtain information on the GDR characteristics (i.e., resonance energy, width and contribution of the GDR to the energy-weighted sum rule (EWSR)) from investigations of the electromagnetic processes of photoabsorption and gamma-decay. However, E1 transitions are dominant near the maximum of the photoabsorption cross sections, but not at lower photon energies closer to the neutron binding energies. There, other physical phenomena like pygmy resonances [4] and scissors mode [5, 6] need to be considered. Those lowenergy collective phenomena have little impact on the nuclear GDR characteristics and are neglected in this work. It can be also noted that collective states like GDR have been extensively studied, both experimentally and theoretically, in atomic physics [7, 8], metallic clusters [7, 9, 10], and quantum-dots [4, 11].

A comprehensive experimental database with reliable data for the GDR parameters and their uncertainties is very important for the reliable modeling of E1 gamma-ray cascades in highly excited nuclei, for the study of nuclear reaction mechanisms as well as for verifying different theoretical approaches used to describe the GDR and other nuclear structure properties (deformations, contribution of velocity-dependent forces, shape-transitions, etc), and forms an integral part of modern nuclear reaction computer codes, such as $[12,13]$. The experimental values of the GDR parameters in cold atomic nuclei are most reliably deduced from experimental photoabsorption cross sections. Compilations of the parameters of Lorentz curves fitted to the total photoneutron cross section data were presented in Refs. [14-16]. The data from Ref.[16] including GDR parameters for light nuclei ${ }^{12} \mathrm{C},{ }^{14} \mathrm{~N},{ }^{16} \mathrm{O},{ }^{27} \mathrm{Al}$ and ${ }^{28} \mathrm{Si}$ were listed in the RIPL-1 database [1] as well as in the RIPL-3 [3] /gamma/gdr-parameters-exp.dat file. Assuming that the contribution of photoproton cross sections to the total photoabsorption cross section is small, then the Lorentzian parameters of the total photoneutron cross sections 
in spherical and axially deformed nuclei can be identified with the GDR parameters. However, note that this assumption is poor for light nuclei where photo-charged particle reactions are important.

Updated tables of GDR parameters with estimates of their uncertainties were subsequently given in Ref. [17]. In this compilation, the GDR parameters were treated as variables in the fitting of calculated total photoabsorption cross sections to the experimental data using the least-squares method.

Comprehensive databases of photonuclear reaction parameters are also published in Refs. [18, 19]. The photoproton contribution was included in the database, but the parameters were obtained without performing any specific fit of an analytical function but were extracted directly by digitizing the characteristics of the experimental data peaks. Microscopic predictions of the GDR energies and widths for about 6000 nuclei between the proton and the neutron drip lines are given in the RIPL-3 database [3]. These GDR parameters resulted from a fit of microscopic calculations of the nuclear gamma-strength function to the existing experimental data [20,21]. The calculations were performed on the basis of the Hartree-Fock-BCS plus quasi-particle random-phase-approximation as well as the microscopic Hartree-FockBogoliubov plus quasi-particle random phase approximation model with a realistic Skyrme interaction.

For heated atomic nuclei, the GDR parameters are determined by gamma-decay data. Compilation and parameterization of the GDR built on excited states are given in Refs. [22, 23].

Tables of updated values of the GDR parameters with estimates of their uncertainties (one-sigma standard deviation) are given in this contribution. This work was performed within an IAEA Coordinated Research Project on Updating the Photonuclear Data Library and generating a Reference Database for Photon Strength Functions [24]. The presented database updates and extends the above-mentioned compilations for the GDR built on cold nuclear states. The parameters are calculated following the prescription of Ref. [17] by fitting Lorentz-like curves to photonuclear cross section data using the least-squares method. For experimental data, the total photoabsorption cross sections retrieved from the EXFOR database [25] or a combination of experimental partial cross sections best suited for approximating the total photoabsorption cross section are used. The evaluated data of [26, 27] are also considered as experimental values. Parameters are given for 144 isotopes from ${ }^{6} \mathrm{Li}$ to ${ }^{239} \mathrm{Pu}$ atomic nuclei and for 19 elements of natural isotopic composition (in total 475 entries).

The updated values of the GDR parameters with their associated uncertainties (one-sigma standard deviation) are given in the Tables 1 and 2. They were obtained by fitting the data with two different models of the GDR excitation: a Standard Lorentzian (SLO) model and a Simplified version of the Modified Lorentzian (SMLO) approach [2, 3, 17, 35] (see below Eqs. (2)-(5)). The updated tables include the following improvements with respect to the previous results reported in Ref.[17]:

- GDR parameters were determined for 164 datasets, including the GDR parameters for 14 new isotopes and 13 natural elements, i.e. for ${ }^{6} \mathrm{Li},{ }^{7} \mathrm{Li},{ }^{9} \mathrm{Be},{ }^{108} \mathrm{Pd},{ }^{112} \mathrm{Sn},{ }^{114} \mathrm{Sn},{ }^{122} \mathrm{Sn},{ }^{151} \mathrm{Eu},{ }^{152} \mathrm{Gd},{ }^{154} \mathrm{Gd},{ }^{158} \mathrm{Gd},{ }^{185} \mathrm{Re},{ }^{203} \mathrm{Tl},{ }^{205} \mathrm{Tl}$ and for ${ }^{\text {nat }} \mathrm{C}$, ${ }^{\text {nat }} \mathrm{Mg}$, ${ }^{\text {nat }} \mathrm{Cu}$, ${ }^{\text {nat }} \mathrm{Rb}$, ${ }^{\text {nat }} \mathrm{Sr},{ }^{\text {nat }} \mathrm{Pd}$, ${ }^{\text {nat }} \mathrm{Ag}$, ${ }^{\text {nat }} \mathrm{Cd}$, ${ }^{\text {nat }} \mathrm{Sb},{ }^{\text {nat }} \mathrm{Ba}$, ${ }^{\text {nat }} \mathrm{Re},{ }^{\text {nat }} \mathrm{Ir},{ }^{\text {nat }} \mathrm{U}$.

- GDR parameters were corrected for 23 datasets by revising the energy intervals of the fitting procedure.

- GDR parameters for 7 datasets, namely for isotopes ${ }^{12} \mathrm{C},{ }^{15} \mathrm{~N}$, ${ }^{\text {nat }} \mathrm{Ar}$, ${ }^{\text {nat }} \mathrm{Fe},{ }^{59} \mathrm{Co}$, ${ }^{\text {nat }} \mathrm{Zr}$ were excluded due to the poor quality of the fit of the experimental data.

Compared to the RIPL-3 Tables [3], the new tables include the following modifications: 
- GDR parameters were added for 271 datasets, including 24 new isotopes that were absent previously, i.e. ${ }^{6} \mathrm{Li},{ }^{7} \mathrm{Li}$, ${ }^{9} \mathrm{Be},{ }^{10} \mathrm{~B},{ }^{65} \mathrm{Zn},{ }^{108} \mathrm{Pd},{ }^{109} \mathrm{Ag},{ }^{112} \mathrm{Sn},{ }^{114} \mathrm{Sn},{ }^{122} \mathrm{Sn},{ }^{151} \mathrm{Eu},{ }^{152} \mathrm{Gd},{ }^{154} \mathrm{Gd},{ }^{158} \mathrm{Gd},{ }^{176} \mathrm{Hf},{ }^{185} \mathrm{Re},{ }^{191} \mathrm{Ir},{ }^{193} \mathrm{Ir},{ }^{194} \mathrm{Pt}$,

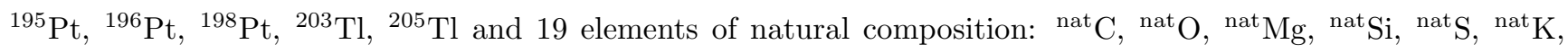

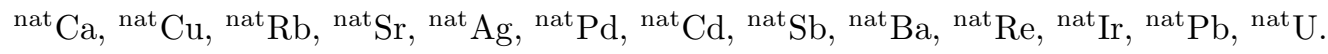

- The GDR values for 40 datasets were corrected by revising the energy interval of fitting and modifying the uncertainty estimates.

- The GDR parameters from 8 datasets, namely ${ }^{15} \mathrm{~N},{ }^{19} \mathrm{~F},{ }^{26} \mathrm{Mg},{ }^{51} \mathrm{~V},{ }^{59} \mathrm{Co},{ }^{127} \mathrm{I},{ }^{141} \mathrm{Pr},{ }^{197}$ Au were excluded due to the poor quality of the fit to experimental data.

Figures 1-162 show the comparison between the experimental photonuclear cross sections and theoretical calculations for a more extended range of gamma-ray energies $(\lesssim 30 \mathrm{MeV})$ compared to those used in the fitting procedure. The theoretical cross sections were calculated using the SLO and SMLO models for the GDR excitation, with parameters taken from Tables 1 and 2, respectively, and including the quasi-deuteron photodisintegration. An estimate of the quality of the description of the experimental data by the two fitted theoretical models is provided by the ratios of the leastsquare deviations (Eq.(11)) of model SMLO to SLO which are calculated for the different gamma-ray energy intervals and are also indicated in the Table $\mathrm{A}$ and in the graphs.

\section{Theoretical basis and assumptions}

A number of different simplified resonance shapes (consisting of one or a sum of the resonance components) is used for the theoretical description of photoabsorption cross sections. The photonuclear data around the peaks of the GDR can be fitted by Lorentz, Breit-Wigner and Gaussian functions equally well, but their low- and high- energy tails are a different matter $[28,29]$. Lorentz $\left(G_{L}\right)$ and Breit-Wigner $\left(G_{B W}\right)$ shapes of the photoabsorption cross sections can be derived from different theoretical approaches [30-35]. The Lorentzian shape can be transformed to Breit-Wigner,

$$
G_{L}(\varepsilon ; E, \Gamma) \equiv \frac{\varepsilon^{2} \Gamma^{2}}{\left(\varepsilon^{2}-E^{2}\right)^{2}+(\varepsilon \Gamma)^{2}}=\frac{\gamma^{2}(\varepsilon) / 4}{(\varepsilon-E)^{2}+\gamma^{2}(\varepsilon) / 4} \equiv G_{B W}(\varepsilon ; E, \gamma(\varepsilon)),
$$

but with shape width $\gamma(\varepsilon)=(2 \varepsilon /(\varepsilon+E)) \Gamma$ which depends on photon energy $\varepsilon$, resonance energy $E$ and width $\Gamma$ of the Lorentzian. In the neighborhood of the resonance energy, the width $\gamma(\varepsilon)$ coincides with $\Gamma$, i.e. $\gamma(\varepsilon \simeq E) \simeq \Gamma$, however on the wings of the resonance it differs. The Breit-Wigner shape $G_{B W}$ with arbitrary energy dependent width is sometimes referred to as the Energy Dependent Breit-Wigner [28].

A Lorentz shape is preferable for fitting the photonuclear data because the standard Breit-Wigner expression is obtained without account for time reversal invariance and is adequate for describing a strong resonance state when the width is small with respect to the resonance energy [31, 32, 34]. These line shapes correspond to a nuclear response function to an electromagnetic field which proceeds through the excitation of one strong collective state that exhausts the EWSR. However, the photoabsorption and gamma-decay processes on the wings of the GDR are governed by the excitation of states of a different nature and therefore, the fitting of experimental data by Lorentzians should be limited to small energy ranges around the GDR peak $[14,16,17]$ in order to obtain reliable values for the GDR parameters. 
In this contribution, the theoretical photoabsorption cross section $\sigma_{a b s}\left(\varepsilon_{\gamma}\right)$ for a photon with energy $\varepsilon_{\gamma}$ is taken as a sum of the terms corresponding to the GDR excitation given by $\sigma_{G D R}\left(\varepsilon_{\gamma}\right)$ and the quasi-deuteron photodisintegration:

$$
\sigma_{a b s}\left(\varepsilon_{\gamma}\right)=\sigma_{G D R}\left(\varepsilon_{\gamma}\right)+\sigma_{Q D}\left(\varepsilon_{\gamma}\right)
$$

where the component $\sigma_{Q D}\left(\varepsilon_{\gamma}\right)$ is a quasi-deuteron contribution, i.e., the photoabsorption cross section on a neutronproton pair $[18,36]$. For a nucleus with $N$ neutrons, $Z$ protons and mass number $A=N+Z$, the latter term is equal to

$$
\sigma_{Q D}\left(\varepsilon_{\gamma}\right)=397.8 \frac{N Z}{A} \frac{\left(\varepsilon_{\gamma}-2.224\right)^{3 / 2}}{\varepsilon_{\gamma}^{3}} \phi\left(\varepsilon_{\gamma}\right)
$$

with $\varepsilon_{\gamma}$ in $\mathrm{MeV}$ and $\sigma_{Q D}$ in units of mb. The function $\phi\left(\varepsilon_{\gamma}\right)$ accounts for the Pauli-blocking of the excited neutron-proton pair in the nuclear medium

$$
\begin{aligned}
\phi\left(\varepsilon_{\gamma}<20 \mathrm{MeV}\right)= & \exp \left(-73.3 / \varepsilon_{\gamma}\right), \\
\phi\left(20<\varepsilon_{\gamma}<140 \mathrm{MeV}\right)= & 8.3714 \times 10^{-2}-9.8343 \times 10^{-3} \varepsilon_{\gamma}+4.1222 \times 10^{-4} \varepsilon_{\gamma}^{2}- \\
& -3.4762 \times 10^{-6} \varepsilon_{\gamma}^{3}+9.3537 \times 10^{-9} \varepsilon_{\gamma}^{4}, \\
\phi\left(\varepsilon_{\gamma}>140 \mathrm{MeV}\right)= & \exp \left(-24.2 / \varepsilon_{\gamma}\right) .
\end{aligned}
$$

The GDR component of the photoabsorption cross section $\sigma_{a b s}\left(\varepsilon_{\gamma}\right)$ is considered to be equal to the photoabsorption cross section of electric dipole (E1) gamma-rays and is calculated within framework of two different Lorentzian models: a Standard Lorentzian (SLO) model and a Simplified version of the Modified Lorentzian (SMLO) approach [2, 3, 17, 35]. For deformed nuclei, an approximation applied to axially deformed nuclei is adopted [2, 3, 17]. However, following Ref. [16], some deformed nuclei are treated as spherical ones, in cases where the fit of the experimental data by the onecomponent Lorentz curve has a lower $\chi^{2}$ per degree of freedom than the fit by a two-component Lorentzian. Assuming axially symmetric shapes, the expression for $\sigma_{G D R}$ is a sum of two Lorentz-like curves corresponding to collective vibrations along and perpendicular to the axis of symmetry $\left(j_{m}=2\right.$ ), respectively (for spherical nuclei $j_{m}=1$ or is omitted)

$$
\begin{gathered}
\sigma_{G D R}\left(\varepsilon_{\gamma}\right)=\sigma_{G D R}^{\alpha}\left(\varepsilon_{\gamma}\right)=\sum_{j=1}^{j_{m}} \sigma_{G D R, j}^{\alpha}\left(\varepsilon_{\gamma}\right)=\sigma_{T R K} s_{j}^{\alpha} \cdot F_{j}^{\alpha}\left(\varepsilon_{\gamma}\right), \\
F_{j}^{\alpha}\left(\varepsilon_{\gamma}\right) \equiv \frac{2}{\pi} \frac{G_{L}\left(\varepsilon_{\gamma} ; E_{r, j}^{\alpha}, \Gamma_{j}^{\alpha}\right)}{\Gamma_{j}^{\alpha}}=\frac{2}{\pi} \frac{\varepsilon_{\gamma}^{2} \Gamma_{j}^{\alpha}}{\left[\varepsilon_{\gamma}^{2}-\left(E_{r, j}^{\alpha}\right)^{2}\right]^{2}+\left[\varepsilon_{\gamma} \Gamma_{j}^{\alpha}\right]^{2}} .
\end{gathered}
$$

Here, $E_{r, j}^{\alpha}, \Gamma_{j}^{\alpha}$ are the resonance energy and shape width, respectively, of the $j$ - mode of the giant dipole excitation for SLO and SMLO models ( $\alpha=S L O$ and SMLO). Factor $s_{j}^{\alpha}$ is the normalized contribution ("weight") of the Lorentzian component $F_{j}^{\alpha}$ of model $\alpha$ to the GDR component of the photoabsorption cross section in terms of the Thomas-Reiche-Kuhn (TRK) sum rule $\sigma_{T R K}$

$$
\sigma_{T R K}=60 \frac{N Z}{A}=15 A\left(1-I^{2}\right)[\mathrm{mb} \cdot \mathrm{MeV}]
$$

where $I=(N-Z) / A$ is the neutron-proton asymmetry factor.

The width $\Gamma_{j}^{S L O}$ does not depend on the gamma-ray energy and coincides with GDR width $\Gamma_{r, j}^{S L O}$, while $\Gamma_{j}^{S M L O}$ is given by a linear function of the gamma-ray energy

$$
\Gamma_{j}^{S L O}=\Gamma_{r, j}^{S L O}=\mathrm{const}, \quad \Gamma_{j}^{S M L O}\left(\varepsilon_{\gamma}\right)=a_{j} \varepsilon_{\gamma}
$$


In the SLO model, the quantities $E_{r, j}^{S L O}, \Gamma_{j}^{S L O}=\Gamma_{r, j}^{S L O}$ and $s_{j}^{S L O}$ are used as variables in the fitting procedure. In the SMLO model, the parameters $E_{r, j}^{S M L O}, a_{j}, s_{j}^{S M L O}$ are determined by fitting, and the widths $\Gamma_{r, j}^{S M L O}$ presented in Table 2 are recalculated from obtained $a_{j}$ by the relationship $\Gamma_{r, j}^{S M L O}=\Gamma_{j}^{S M L O}\left(\varepsilon_{\gamma}=E_{r, j}^{S M L O}\right)=a_{j} E_{r, j}^{S M L O}$.

A resonance value $\sigma_{r, j}^{\alpha}=\sigma_{G D R, j}^{\alpha}\left(\varepsilon_{\gamma}=E_{r, j}^{\alpha}\right)$ of the cross section component corresponding to the giant dipole vibration along the $j$-axis at resonance energy $\varepsilon_{\gamma}=E_{r, j}^{\alpha}$ is equal to:

$$
\sigma_{r, j}^{\alpha}=\frac{2}{\pi} \sigma_{T R K} s_{j}^{\alpha} / \Gamma_{r, j}^{\alpha},
$$

where $\Gamma_{r, j}^{\alpha}=\Gamma_{j}^{\alpha}\left(\varepsilon_{\gamma}=E_{r, j}^{\alpha}\right)$ is width of $j$-component at corresponding resonance energy.

If integral of function $F_{j}^{\alpha}$ over energy is equal to unity, $F_{j}^{\alpha, i n t}=\int_{0}^{\infty} F_{j}^{\alpha}\left(\varepsilon_{\gamma}\right) d \varepsilon_{\gamma}=1$, the sum $s^{\alpha}=s_{1}^{\alpha}+s_{2}^{\alpha}$ of the weights in Eq.(5) is, by definition, the energy weighted sum-rule in units of the TRK sum rule $\sigma_{T R K}$, and $s_{j}^{\alpha}$ is the strength of the corresponding mode of the giant dipole excitation. The $s^{\alpha}$ should be not exceed a value $1+\Delta$,

$$
s^{\alpha}=\sum_{j} s_{j}^{\alpha} \leq 1+\Delta, \quad \Delta \approx 0.2 \div 0.3
$$

where $\Delta$ is the contribution of the velocity-dependent and exchange forces [37]. Note that the GDR exhausts the EWSR by $84 \%$ only within the framework of the hydrodynamic approach to SLO model [33].

In the approximation of equally probable excitation of normal modes of the giant collective vibration, the weights $s_{j}=s_{j}^{\alpha}$ are equal to

$$
\left\{\begin{array}{l}
s_{1}=s / 3 ; \quad s_{2}=2 s / 3, \quad \beta>0, \\
s_{1}=2 s / 3 ; \quad s_{2}=s / 3, \quad \beta<0
\end{array}\right.
$$

because of twofold degeneration of the giant collective vibration, which is perpendicular to the axis of symmetry; $s=s^{\alpha}$.

The $\beta$ is a parameter of quadrupole deformation and the subindex $j=1(2)$ in $s_{j}$ corresponds to the low (high) value component of the GDR energy, respectively, i.e. $E_{r, j=1}^{\alpha}<E_{r, j=2}^{\alpha}$.

It should be mentioned that in SLO model $F_{j}^{S L O, i n t}=1$ and the weights $s_{j}$ are the strengths of the GDR excitation modes in units of the TRK sum rule. For the SMLO and other models with energy-increasing width, $F_{j}^{\alpha, i n t} \neq 1$ and the factors $s_{j}$ can be interpreted to be the GDR strengths only approximately.

In this work a least-squares fitting procedure was employed, in which the data points were weighted according to the inverse square of their uncertainties and a minimum value was sought for a least-square deviation $\chi_{\text {model }}^{2}$ :

$$
\chi_{\text {model }}^{2}=\frac{1}{N_{f}} \sum_{i=1}^{N} \frac{\left(\sigma_{\exp }\left(\varepsilon_{i}\right)-\sigma_{a b s}^{\text {model }}\left(\varepsilon_{i}\right)\right)^{2}}{\left(\Delta \sigma\left(\varepsilon_{i}\right)\right)^{2}},
$$

where $\sigma_{a b s}^{\text {model }}\left(\varepsilon_{i}\right)$ is the value of the theoretical cross section (2)-(5) with $\sigma_{G D R}\left(\varepsilon_{i}\right)$ for model SLO or SMLO at gammaray energy $\varepsilon_{i}, \sigma_{\exp }\left(\varepsilon_{i}\right)$ is the measured value for the total photoabsorption cross section with uncertainty $\Delta \sigma\left(\varepsilon_{i}\right)$ at that energy, and $N_{f}=N-N_{p a r}$ is the number of degrees of freedom for the data set fitted which is equal to the number $N$ of data points within the fitting interval minus the number $N_{\text {par }}$ of fitted parameters (3 parameters for each Lorentz-like curve).

If the EXFOR datafiles did not include experimental uncertainties, then they were taken into account according to Ref. [17]. Specifically, two different estimations of errors are adopted in absence of experimental uncertainties to check their impact on the determination of the GDR parameters: a 10\% constant error and an energy-dependent error. The former type of error is very often encountered in photoabsorption data files in the EXFOR database, while the latter energy dependence was chosen to simulate the statistical error that is inversely proportional to the counting rate which 
is maximum near the GDR. Hence, the energy-dependent relative uncertainties were assumed to take minimum values (10\%) near the GDR peaks and maximum values (50\%) on the GDR tails. For spherical nuclei, a triangular dependence on gamma-energy was assumed, while for deformed nuclei a trapezoidal dependence with the GDR peaks as the top corners of the trapezium.

For a given nuclide the data were fitted in an energy interval that was determined following the prescription of Refs. [14-19]. The mid-position was chosen near the peak of the photoabsorption cross section and the interval was placed within an energy range defined by the one-neutron and three-neutron separation energies, where the GDR strength is expected to be dominant. Adjustments to this interval were made in cases where there were too few experimental data points, in order to allow for a good fit to be obtained using a Lorentzian curve.

The minimization of the least-square deviation functional was performed by the CERN MINUIT package [38]. The standard deviation of the parameters was estimated using the MINOS procedure of this code. The calculated mode was defined by the following sequence of commands: SEEK 1000, MIGRAD 10000 0.000001, IMPROVE 100, HESSE 1, MINOS 1.

\section{Discussion and Conclusion}

It should be noted that the energies $E_{r, j}$, widths $\Gamma_{r, j}$ and factors $s_{j}$ presented in Tables 1 and 2 are, in fact, the parameters of Lorentz-like curves (Lorentz parameters) representing the best fit of the experimental photoabsorption cross sections in the intervals that hold peak energies for the SLO and SMLO models, respectively.

Different studies mentioned in the previous section demonstrate that the Lorentzian parameters can be considered as the GDR parameters or mean values of these parameters if the total photoabsorption cross-section is used in fitting and GDR excitation is dominant photoabsorption mechanism.

The accuracy of the description of GDR parameters by Lorentz parameters of the photoabsorption cross section data depends on many factors. Besides the well-known problems of the selection and verification of the experimental data and estimating the contributions of emission cross sections of different particles to the total photoabsorption cross section, there are also ambiguities in the theoretical description of the GDR component of the E1 photoabsorption cross section. Namely, an unsolved problem with description of nuclear dissipation as well as the approximation of $\sigma_{G D R}$ by one- or two-component Lorentz-like curves (5) near the beta-stability valley is, as a rule, appropriate for rather heavy $(A \gtrsim 40)$ spherical and axially deformed nuclei $(155 \lesssim \mathrm{A} \lesssim 190$ or $225 \lesssim \mathrm{A} \lesssim 250)$ for gamma-ray energies close to the GDR energy.

Furthermore, additional physical effects should also be taken into account for the correct description of the photoabsorption in nuclei. The nuclear responses of the low-lying states (two-phonon states, pygmy dipole resonance) to the E1 field have an impact on the photoabsorption at the low-energy wing of the GDR. The isospin splitting, the neutron excess and the triaxial deformation can affect the shape of the photoabsorption cross section near the GDR and lead to the splitting of the GDR. For medium-mass to heavy nuclei, these effects can be described effectively by an additional broadening of the shape-line. But in some situations, isospin splitting and triaxiality of the nuclear shape have to be considered explicitly in the formulas [28, 39-43].

From the tables 1,2 it can be seen that these uncertainties for GDR energy $E_{r}$ do not exceed $\sim 10 \%$. The values of width $\Gamma_{r}$ and the factors $s_{j}$ are much more sensitive to the shape of the theoretical curve and their systematic 
uncertainties can be rather significant. The difference in the magnitude of the parameters obtained from fitting within SLO and SMLO models can be considered as the systematic uncertainty due to ambiguity of the theoretical description of complex mechanism of the GDR relaxation which as yet studied. However, the overall consistency between different sets of parameters for the same nucleus in each table are, as a rule, rather good, while the variation of these parameters can be attributed to systematic uncertainties affecting both measurements and the theoretical approach.

The set of the Lorentzian parameters in the first line (first entry) for each nuclide was determined using, as a rule, the closest experimental data to the total photoabsorption cross sections near GDR and corresponds to minimal least-square deviation in comparison with other entries. Therefore, the quantities in the first entries are the recommended GDR parameters and are used in all our calculations presented below.

The quality of the description of the experimental photonuclear cross sections by the different models is considered by means of the ratio of the least-square deviations (Eq.(11)) for models SMLO and SLO, $R c_{i}=\chi_{\text {smlo }}^{2} / \chi_{\text {slo }}^{2}$, in different intervals $\left(\Delta \varepsilon_{i}\right)$. The lower index $i$ indicates the comparison interval: $i=f$ stands for fitting intervals $\Delta \varepsilon_{f}=\varepsilon_{m i n} \leq$ $\varepsilon_{\gamma} \leq \varepsilon_{\max }$ from the Tables 1,$2 ; i=l$ for intervals of the GDR left tail $\Delta \varepsilon_{l}=\varepsilon_{0} \leq \varepsilon_{\gamma} \leq E_{r, 1}$ and $i=m$ for whole intervals with the experimental data $\Delta \varepsilon_{m}=\varepsilon_{0} \leq \varepsilon_{\gamma} \leq 30 \mathrm{MeV}$ with the energy $\varepsilon_{0}$ of the first experimental data-point.

The factors $R c_{i}$ are indicated in the Graphs and are collected in the Table A. This table gives the ratios of the arithmetic means of least-square values of models SMLO to SLO $<\chi_{\text {smlo }}^{2}>/<\chi_{\text {slo }}^{2}>$ in different gamma-ray intervals and for different sets of nuclei. Here, the isotopes from the mass-ranges $150 \leq \mathrm{A} \leq 190$ and $220 \leq \mathrm{A} \leq 253$ are named as axially deformed nuclei. The parameters $\beta$ of effective quadrupole deformation of nuclear surface were taken from file "deflib.dat" packed in "gamma-strength-analytic.tgz", section GAMMA on http://www-nds.iaea.org/RIPL-2/. Nuclei with $\beta<0.1$ were assumed to be spherical.

Table A

The ratio of arithmetical means of least-square deviations for SMLO and SLO models $<\chi^{2}(S M L O)>/<\chi^{2}(S L O)>$

\begin{tabular}{|c|c|c|c|}
\hline \multirow{2}{*}{ Intervals } & \multicolumn{3}{|c|}{ Nuclei } \\
\cline { 2 - 4 } & Spherical & Spherical+Axially deformed & All \\
\hline$\Delta \varepsilon_{f}$ & 0.96 & 1.11 & 1.09 \\
\hline$\Delta \varepsilon_{l}$ & 0.71 & 0.73 & 0.76 \\
\hline$\Delta \varepsilon_{m}$ & 0.70 & 0.69 & 0.74 \\
\hline
\end{tabular}

The curves in the graphs demonstrate that the low-energy tails of the photoabsorption cross sections within SLO model are, as a rule, higher than the SMLO model and the experimental data. This means that excitations of low-energy states (like pygmy dipole resonance) can not be described as additional peaks to SLO model without re-adjusting the standard values of its GDR parameters that provided description of the GDR range of the photoabsorption cross sections.

As mentioned above, SMLO model is based on nuclear response with the GDR excitation and energy-dependent width $\Gamma\left(\varepsilon_{\gamma}\right)$ that is linearly increasing with gamma-ray energy. The expressions for energy-dependent widths whose energy dependence increases with energy are based on a low-energy approximation of nucleon-nucleon collision cross section in the nuclear medium $[44,45]$ and are not valid at high energies $[46,47]$. Therefore SMLO model can not 
be used without modifications of the width above high gamma-ray energies ( 25-30 MeV) where the QD component is dominant.

\section{Acknowledgments}

The authors are very thankful to V.Yu. Denisov, S. Goriely, P. Oblozinský, M.W. Herman , A.V. Ignatyuk, J. Kopecky, and V.V. Varlamov for valuable discussions and comments. We very much appreciate help provided by Katerina Solodovnik in preparing the datafiles. This work is partially supported by the International Atomic Energy Agency through a Coordinated Research Project on Updating the Photonuclear Data Library and generating a Reference Database for Photon Strength Functions (F41032).

\section{References}

[1] P. Oblozinský, M. B. Chadwick, T. Fukahori, A.V. Ignatyuk, S. Kailas, J. Kopecky, G. Molnar, G. Reffo, Z. Su, M. Uhl, P. G. Young, O. Bersillon, E. Běták, R. Capote and V. M. Maslov, Handbook for Calculations of Nuclear Reaction Data: Reference Input Parameter Library), Tech. Rep. IAEA-TECDOC-1034, International Atomic Energy Agency, Vienna, Austria, 1998 (see http://www-nds.iaea.org/ripl/).

[2] T. Belgya, O. Bersillon, R. Capote, T. Fukahori, Zhigang Ge, S. Goriely, M. Herman, A. V. Ignatyuk, S. Kailas, A. Koning, V. Plujko and P. Young, Handbook for calculations of nuclear reaction data, Reference Input Parameter Library- 2, Tech. Rep. IAEA-TECDOC-1506, International Atomic Energy Agency, Vienna, Austria, 2006(see http://www-nds.iaea.org/RIPL-2/).

[3] R. Capote, M. Herman, P. Oblozinský, P. G. Young, S. Goriely, T. Belgya, A. V. Ignatyuk, A. J. Koning, S. Hilaire, V. A. Plujko, M. Avrigeanu, O. Bersillon, M. B. Chadwick, T. Fukahori, Zhigang Ge, Yinlu Han, S. Kailas, J. Kopecky, V. M. Maslov, G. Reffo, M. Sin, E. Sh. Soukhovitskii and P. Talou, Nucl. Data Sheets 110 (2009) 3107; (see http://www-nds.iaea.org/RIPL-3/).

[4] R. Capote, A. Delgado, and A. Gonzalez, Mod. Phys. Lett. B15 (2001) 81.

[5] J. L. Ullmann, T. Kawano, T. A. Bredeweg, A. Couture, R. C. Haight, M. Jandel, J. M. ODonnell, R. S. Rundberg, D. J. Vieira, J. B. Wilhelmy, J. A. Becker, A. Chyzh, C. Y. Wu, B. Baramsai, G. E. Mitchell, and M. Krticka, Phys. Rev. C89 (2014) 034603.

[6] M. R. Mumpower, T. Kawano, J. L. Ullmann, M. Krticka, and T. M. Sprouse, Phys. Rev. C96 (2017) 024612.

[7] Review articles can be found in Correlations in Clusters and Related Systems, ed. J. P. Connerade (World Scientific, Singapore, 1996).

[8] E. Lipparini, in Many Body Theory of Correlated Electron Systems, eds. M. I. Gallardo and M. Lozano (World Scientific, Singapore, 1998).

[9] S. Pollach, C. R. C. Wang and M. M. Kappes, J. Chem. Phys. 94 (1991) 2496.

[10] G. F. Bertsch and R. A. Broglia, Oscillations in Finite Quantum Systems (Cambridge University Press, Cambridge, 1994). 
[11] A. Delgado, L. Lavin, R. Capote and A. Gonzalez, Physica E8 (2000) 345.

[12] M. Herman, R. Capote, B. V. Carlson, P. Oblozinský, M. Sin, A. Trkov, H. Wienke and V. Zerkin, Nucl. Data Sheets 108 (2007) 2655 .

[13] A. J. Koning, S. Hilaire and M. C. Duijvestijn, TALYS-1.0, Proceedings of the International Conference on Nuclear Data for Science and Technology, April 22-27, 2007, Nice, France, editors O.Bersillon, F.Gunsing, E.Bauge, R.Jacqmin, and S.Leray, EDP Sciences, 2008, p. 211-214 (see www.talys.eu).

[14] B. L. Berman and S. C. Fultz, Rev. Mod. Phys. 47 (1975) 713.

[15] B. L. Berman, R. G. Pywell, S. S. Dietrich, M. N. Thompson, K. O. McNeill and J. W. Jury, Phys. Rev. C36 (1987) 1286.

[16] S. S. Dietrich and B. L. Berman, At. Data Nucl. Data Tables 38 (1988) 199.

[17] V. A. Plujko, R. Capote, O. M. Gorbachenko. At. Data Nucl. Data Tables, 97 (2011) 567.

[18] M. B. Chadwick, P. Oblozinský, A. I. Blokhin, T. Fukahori, Y. Han, Y. -O. Lee, M. N. Martins, S. F. Mughabghab, V. V. Varlamov, B. Yu and J. Zhang, Handbook on photonuclear data for applications: Cross sections and spectra, Tech. Rep. IAEA-TECDOC-1178, (International Atomic Energy Agency, Vienna, Austria, 2000) (see http://wwwnds.iaea.org/reports-new/tecdocs/iaea-tecdoc-1178.pdf).

[19] A. V. Varlamov, V. V. Varlamov, D. S. Rudenko and M. E. Stepanov, Atlas of Giant Dipole Resonances. Parameters and graphs of photonuclear reaction cross sections, Tech. Rep. INDC(NDS)-394, (International Atomic Energy Agency, Vienna, Austria, 1999) (see http://www-nds.iaea.org/reports-new/indc-reports/indc-nds/indc-nds0394.pdf).

[20] S. Goriely and E. Khan, Nucl. Phys. A706 (2002) 217.

[21] S. Goriely, E. Khan and M. Samyn, Nucl. Phys. A739 (2004) 331.

[22] A. Schiller and M. Thoennessen, At. Data Nucl. Data Tables 93 (2007) 549.

[23] D. Kusnezov, Y. Alhassid, K. A. Snover and W. E. Ormand, Nucl. Phys. A687 (2001) 12.

[24] S.Goriely and P. Dimitriou (Prep.). Summary Report 1st RCM on CRP "Updating Photonuclear Data Library and Generating a Reference Database for Photon Strength Functions", INDC(NDS)-0712, Vienna, 2016.

[25] International Network of Nuclear Reaction Data Centres: EXFOR/CSISRS database (see http://wwwnds.iaea.org/exfor/).

[26] V. V. Varlamov, B. S. Ishkhanov, V. N. Orlin, , K. A. Stopani, Europian Phys. Jour. A, 51 (2015) 67.

[27] V. V. Varlamov, B. S. Ishkhanov, V. N. Orlin, N. N. Peskov, Yad. Fiz. 79 (4) (2016) 315.

[28] D. G. Gardner, In: Neutron Radiative Capture. (Neutrons physics and nuclear data in science and technology; Vol.3)), Gen. Eds.: A. Michaudon, S. Cierjacks, R. E. Chrien, Pergamon Press, Oxford, New York, $1984 ;$ pp.62-118.

[29] G. A. Bartholomew, E. D. Earle, A. J. Ferguson, J. W. Knowles,and M. A. Lone, Advances Nucl. Phys., 7 (1973) 229 .

[30] D. M. Brink, Ph. D. Thesis, Oxford University, 1955. 
[31] M. Danos, W. Greiner, Phys. Lett., 8 (1964) 113.

[32] M. Danos,W. Greiner, Phys. Rev., 138B (1965) 876.

[33] J. M. Eisenberg, W. M. Greiner, Nuclear Theory. Volume I: Nuclear Models, 3rd Ed. (North Holland, Amsterdam 1987).

[34] C. B. Dover, R. H. Lemmer, F. J. W. Hahne, Ann. Phys. 70 (1972) 458.

[35] V. A. Plujko, I. M. Kadenko, O. M. Gorbachenko and E. V. Kulich, Int. J. Mod. Phys. E17 (2008) 240.

[36] M. B. Chadwick, P. Oblozinský, P. E. Hodgson and G. Reffo, Phys. Rev. C44 (1991) 814.

[37] E. Lipparini and S. Stringari, Phys. Rep. 175 (1989) 103.

[38] CERN Program Library, MINUIT (D506), Function Minimization and Error Analysis; code available online at http://wwwasdoc.web.cern.ch/wwwasdoc/cernlib.html; user manual available online at http://wwwasdoc.web.cern.ch/wwwasdoc/minuit/minmain.html.

[39] T. J. Bowles, R. J. Holt, H. E. Jackson, R. M. Laszewski, R. D. McKeon, A. M. Nathan, J. R. Specht, Phys. Rev. C24 (1981) 1940.

[40] Y. Alhassid, B. Busch, S. Levit, Phys. Rev. Lett., 61 (1988) 1926.

[41] Y. Alhassid, B. Busch, Nucl. Phys. A509 (1990) 461.

[42] A. R. Junghans, G. Rusev, R. Schwengner, A. Wagner and E. Grosse, Phys. Lett. B670 (2008) 200.

[43] E. Grosse, A. R. Junghans, R. Massarczyk, Physics Letters B 739 (2014) 425.

[44] V. M. Kolomietz, V. A. Plujko and S. Shlomo, Phys. Rev. C54 (1996) 3014.

[45] V. A. Plujko, O. M. Gorbachenko and M. O. Kavatsyuk, Acta Phys. Slov. 51 (2001) 231.

[46] C. Kalbach, Phys. Rev., C33 (1986) 818.

[47] A. J. Koning, M. C. Duijvestijn; Nucl. Phys. A 744 (2004) 15. 


\section{Explanation of Tables}

Table 1. Experimental values and uncertainties of GDR parameters within the standard Lorentzian (SLO) approach

For experimental data in the fits are used the following cross sections: the total photoabsorption reaction cross section $\sigma(\gamma, a b s)$, total photoneutron cross section $\sigma(\gamma, s n)$ and inclusive photoneutron yield cross section $\sigma(\gamma, x n)$ which includes the multiplicity of neutrons emitted in each reaction event:

$$
\begin{gathered}
\sigma(\gamma, a b s)=\sigma(\gamma, s n)+\sigma(\gamma, c p) \\
\sigma(\gamma, s n)=\sigma(\gamma, n)+\sigma(\gamma, n p)+\sigma(\gamma, 2 n)+\sigma(\gamma, 2 n p)+\sigma(\gamma, 3 n)+\ldots+\sigma(\gamma, F), \\
\sigma(\gamma, c p)=\sigma(\gamma, p)+\sigma(\gamma, 2 p)+\ldots+\sigma(\gamma, d)+\sigma(\gamma, d p)+\ldots+\sigma(\gamma, \alpha)+\ldots . \\
\sigma(\gamma, x n)=\sigma(\gamma, 1 n x)+2 \sigma(\gamma, 2 n x)+3 \sigma(\gamma, 3 n x)+\ldots+\bar{\nu} \sigma(\gamma, F),
\end{gathered}
$$

where $\sigma(\gamma, c p)$ is the photo-charged-particle reaction cross section, $\bar{\nu}$ is the average multiplicity of photofission neutrons.

When measured and evaluated data on the total photoabsorption cross section for a given nuclide were absent in the database, the total photoneutron cross section $\sigma(\gamma, s n)$ was taken instead of the photoabsorption cross section $\sigma(\gamma, a b s)$. Such an approximation is valid if the contribution of the photo-charged-particle reaction cross sections is small. In the absence of experimental EXFOR data for the $\sigma(\gamma, s n)$, the total photoneutron cross section was evaluated as a combination of the available experimental cross sections for the inclusive photoneutron yield cross section $\sigma(\gamma, x n)$ and photoneutron cross sections with ejection of more than one neutron. It is denoted as $\sigma_{\text {Id }}(\gamma, s n)$ with "Id" for type of experimental data used in fitting (see below). For experimental data without uncertainties in the EXFOR base, the relative uncertainties were used either with constant value of $10 \%$ or as the energy-dependent quantities with minimal values (10\%) near the GDR peaks and maximal values (50\%) on the GDR tails as described in Ref.[17] and Sect.2. Throughout the table the italicized numbers refer to the uncertainties in the last digits of the quoted values.

The following designations are used in the table. 

Type of experimental data used in fitting:

0 - experimental $\sigma(\gamma, a b s)$ with experimental uncertainties;

1a - experimental $\sigma(\gamma, a b s)$ with constant uncertainties (10\%);

$1 \mathrm{~b}$ - experimental $\sigma(\gamma, a b s)$ with energy-dependent uncertainties;

2 - evaluated $\sigma(\gamma, a b s)$ with experimental uncertainties;

3a - evaluated $\sigma(\gamma, a b s)$ with constant uncertainties (10\%);

$3 \mathrm{~b}$ - evaluated $\sigma(\gamma, a b s)$ with energy-dependent uncertainties;

4 - experimental $\sigma(\gamma, s n)$ with experimental uncertainties;

4a - experimental $\sigma(\gamma, s n)$ with constant uncertainties (10\%);

$4 \mathrm{~b}$ - experimental $\sigma(\gamma, s n)$ with energy-dependent uncertainties;

5 - evaluated $\sigma(\gamma, s n)$ using experimental data with experimental uncertainties;

6 - composed $\sigma(\gamma, s n)$ as a combination of selected experimental cross sections:

$\sigma_{6}(\gamma, s n)=(\sigma(\gamma, x n)+\sigma(\gamma, 1 n)) / 2$ with absolute uncertainties:

$\Delta \sigma_{6}(\gamma, s n)=\sqrt{\Delta \sigma^{2}(\gamma, x n)+\Delta \sigma^{2}(\gamma, 1 n)} / 2 ;$

7 - composed $\sigma(\gamma, s n)$ as a combination of the experimental cross sections:

$\sigma_{7}(\gamma, s n)=\sigma(\gamma, x n)-\sigma(\gamma, 2 n)$ with absolute uncertainties:

$\Delta \sigma_{7}(\gamma, s n)=\sqrt{\Delta \sigma^{2}(\gamma, x n)+\Delta \sigma^{2}(\gamma, 2 n)} ;$

8 - composed $\sigma(\gamma, s n)$ as a combination of selected experimental cross sections:

$\sigma_{8}(\gamma, s n)=\sigma(\gamma, 1 n)+\sigma(\gamma, 2 n)$ with absolute uncertainties:

$\Delta \sigma_{8}(\gamma, s n)=\sqrt{\Delta \sigma^{2}(\gamma, 1 n)+\Delta \sigma^{2}(\gamma, 2 n)}$

$8 \mathrm{a}$ - experimental $\sigma_{8}(\gamma, s n)$ with constant uncertainties (10\%);

$8 \mathrm{~b}$ - experimental $\sigma_{8}(\gamma, s n)$ with energy-dependent uncertainties;

9 - composed $\sigma(\gamma, s n)$ as a combination of selected experimental cross sections:

$\sigma_{9}(\gamma, s n)=\sigma(\gamma, 1 n)+\sigma(\gamma, 2 n)+\sigma(\gamma, 3 n)$ with absolute uncertainties :

$\Delta \sigma_{9}(\gamma, s n)=\sqrt{\Delta \sigma^{2}(\gamma, 1 n)+\Delta \sigma^{2}(\gamma, 2 n)+\Delta \sigma^{2}(\gamma, 3 n)} ;$

10 - composed $\sigma(\gamma, s n)$ as a combination of selected experimental cross sections:

$\sigma_{10}(\gamma, s n)=\sigma(\gamma, 1 n)+\sigma(\gamma, 2 n)+\sigma(\gamma, f)$ with absolute uncertainties:

$\Delta \sigma_{10}(\gamma, s n)=\sqrt{\Delta \sigma^{2}(\gamma, 1 n)+\Delta \sigma^{2}(\gamma, 2 n)+\Delta \sigma^{2}(\gamma, f)} ;$

11 - composed $\sigma(\gamma, s n)$ as a combination of selected experimental cross sections:

$\sigma_{11}(\gamma, s n)=(\sigma(\gamma, x n)+\sigma(\gamma, 1 n)+\sigma(\gamma, f)) / 2$ with absolute uncertainties:

$\Delta \sigma_{11}(\gamma, s n)=\sqrt{\Delta \sigma^{2}(\gamma, x n)+\Delta \sigma^{2}(\gamma, 1 n)+\Delta \sigma^{2}(\gamma, f)} / 2 ;$

12 - experimental $\sigma(\gamma, 1 n)$ with experimental uncertainties.

13 - experimental $\sigma(\gamma, x n)$ with experimental uncertainties; 
Table 1 (continued)
$E_{r, j}, \Gamma_{r, j}, s_{j} \quad$ parameters of energy, width and weight of Lorentz curves fitted to the corresponding photoabsorption cross sections within the indicated fitting interval. Notation 'spherical' $(j=1)$ implies that a one component Lorentz curve gives a better fit to the data that a two-component one, and 'axially deformed' $(j=1,2)$ implies the opposite; the values of TRK sum rule for ${ }^{208} \mathrm{~Pb}$ are used for ${ }^{\text {nat }} \mathrm{Pb}$.
$E_{r, 1}$ energy of the first component of the GDR with uncertainty (one-sigma standard deviation), MeV.
$\Gamma_{r, 1}$ width of the first component of the GDR with uncertainty (one-sigma standard deviation), MeV.
$s_{1}$ weight of the first component of the GDR with uncertainty (one-sigma standard deviation).
$E_{r, 2}$ energy of the second component of the GDR with uncertainty (one-sigma standard deviation), MeV.
$\Gamma_{r, 2}$ width of the second component of the GDR with uncertainty (one-sigma standard deviation), MeV.
$s_{2}$
$s$ weight of the second component of the GDR with uncertainty (one-sigma standard deviation). sum of weights of the first and second component of the GDR $\left(s=s_{1}+s_{2}\right)$ with uncertainty (one-sigma standard deviation).
$\varepsilon_{\min }\left(\varepsilon_{\max }\right) \quad$ lower (upper) energy limit of fitting interval, $\mathrm{MeV}$.
Ref Short references on the experimental data used in the fit.

Table 2. Experimental values and uncertainties of GDR parameters within the modified Lorentzian (SMLO) approach

Same as for Table 1.

Table 3. References to experimental and evaluated cross section data taken from EXFOR

Nucl

Id

The target studied (symbol); nat supra-index indicates a natural isotopic composition.

Reaction

Ref

EXFOR
Type of experimental data used in fitting (without a letter indicating the method of uncertainty estimation employed; with the letters a, b : Ida - calculations with constant uncertainties (10\%) and Idb - calculations with energy-dependent uncertainties). See the explanation for Table 1. Type of reaction. Short references on the experimental data. EXFOR 8-digit entry \& subentry number. 


\section{Explanation of Graphs}

In the graphs presented comparison of experimental photonuclear cross sections near the GDR region from EXFOR database and theoretical calculations for the nuclei from ${ }^{6} \mathrm{Li}$ to ${ }^{239} \mathrm{Pu}$.

For experimental data, the photoabsorption cross section $\sigma(\gamma, a b s)$ and total photoneutron cross section $\sigma(\gamma, s n)$ were used as it is described in the section "Explanation of Tables". Short reference for experimental data is indicated in the graphs in accordance with the section "References used in the Tables". The type of experimental data (ID index) for given nuclide corresponds to that in the first line of the Tables 1,2 . These markers allow to locate entry and subentry numbers of EXFOR datafile by the use of information in the Table 3.

The theoretical calculations were performed using Eqs. (2)-(5) with allowance for components of the GDR excitation within SLO and SMLO models and contribution of quasi-deuteron photodisintegration. The theoretical curves are shown for more extended range of gamma-ray energies from 5 to $30 \mathrm{MeV}$ when compared to fitting intervals.

The following designations are used in the graphs.

Title descriptor above the figure.

Notations of the photonuclear reaction type and irradiated target nucleus.

Coordinate axes.

Incident gamma-ray energy in units of $\mathrm{MeV}$ is indicated as an abscissa; the cross section in units of $m b$ is indicated as an ordinate.

\section{Vertical short lines.}

Two lines normally to the $x$ - coordinate show the energy interval $\varepsilon_{\gamma}$ from $\varepsilon_{\min }$ to $\varepsilon_{\max }$ were fitting procedure was performed.

$R c_{f}, R c_{l}, R c_{m}$.

The $R c_{i}=\chi_{\text {smlo }}^{2} / \chi_{\text {slo }}^{2}$ is ratio of the least-square deviations (Eq.(11)) in interval $\left(\Delta \varepsilon_{i}\right)$ indexed by $i: i=f$ stands for fitting intervals $\Delta \varepsilon_{f}=\varepsilon_{\min } \leq \varepsilon_{\gamma} \leq \varepsilon_{\max }$ from the Tables 1,$2 ; i=l$ for intervals of the GDR left tail $\Delta \varepsilon_{l}=$ $\varepsilon_{0} \leq \varepsilon_{\gamma} \leq E_{r, 1}$ and $i=m$ for whole intervals with the experimental data $\Delta \varepsilon_{m}=\varepsilon_{0} \leq \varepsilon_{\gamma} \leq 30 \mathrm{MeV}$ with the energy $\varepsilon_{0}$ of the first experimental data-point. 


\section{References used in the Tables}

[1958Ful] E. G. Fuller, M. S. Weiss, Phys. Rev. 112 (1958) 560.

[1962Bog] O. V. Bogdankevich, B. I. Goryachev, V. A. Zapevalov, Zhur. Eksper. Teoret. Fiz. 42 (1962) 150 (Sov. Phys. JETP 15 (1962) 1044).

[1962Fu1] S. C. Fultz, R. L. Bramblett, J. T. Caldwell, N. E. Hansen, C. P. Jupiter, Phys. Rev. 128 (1962) 2345.

[1962Fu2] S. C. Fultz, R. L. Bramblett, J. T. Caldwell, N. A. Kerr, Phys. Rev. 127 (1962) 1273.

[1963Br1] R. L. Bramblett, J. T. Caldwell, G. F. Auchampaugh, S. C. Fultz, Phys. Rev. 129 (1963) 2723.

[1963Bur] N. A. Burgov, G. V. Danilyan, B. S. Dolbilkin, L. E. Lazareva, F. A. Nikolaev, Zhur. Eksper. Teoret. Fiz. 45,(6) (1963) 1694 (Sov. Phys. JETP 43(1) (1963) 50).

[1964Baz] E. B. Bazhanov, A. P. Komar, A. V. Kulikov, Zhur. Eksper. Teoret. Fiz. 46 (1964) 1497 (Sov. Phys. JETP 19 (1964) 1014).

[1964Br1] R. L. Bramblett, J. T. Caldwell, R. R. Harvey, S. C. Fultz, Phys. Rev. 133 (1964) B869.

[1964Fu1] S. C. Fultz, R. L. Bramblett, J. T. Caldwell, R. R. Harvey, Phys. Rev. 133 (1964) B1149.

[1964Ha2] R. R. Harvey, J. T. Caldwell, R. L. Bramblett, S. C. Fultz, Phys. Rev. 136 (1964) B126.

[1964Ric] L. B. Rice, L. N. Bolen, W. D. Whitehead, Phys. Rev. 134 (1964) B557.

[1965Be1] B. L. Berman, R. L. Bramblett, J. T. Caldwell, R. R. Harvey, S. C. Fultz, Phys. Rev. Lett. 15 (1965) 727.

[1965Wyc] J. M. Wyckoff, B. Ziegler, H. W. Koch, R. Uhlig, Phys. Rev. 137 (1965) B576.

[1966Axe] P. Axel, J. Miller, C. Schuhl, G. Tamas, C. Tzara, J. Phys. (Paris) 27 (1966) 262.

[1966Br1] R. L. Bramblett, J. T. Caldwell, B. L. Berman, R. R. Harvey, S. C. Fultz, Phys. Rev. 148 (1966) 1198.

[1966Dol] B. S. Dolbilkin, V. A. Zapevalov, V. I. Korin, L. E. Lazareva, F. A. Nikolaev, Izv. Akad. Nauk SSSR, Ser. Fiz 30(2) (1966) 349 (Bull. Acad. Sci. USSR, Phys. Ser. 30(2) (1966) 354).

[1966Fu1] S. C. Fultz, J. T. Caldwell, B. L. Berman, R. L. Bramblett, R. R. Harvey, Phys. Rev. 143 (1966) 790.

[1967Ant] G. P. Antropov, I. E. Mitrofanov, B. S. Russkikh Izv. Akad. Nauk SSSR, Ser. Fiz 31 (1967) 336 (Bull. Acad. Sci. USSR, Phys. Ser. 31 (1968) 320).

[1967Be2] B. L. Berman, J. T. Caldwell, R. R. Harvey, M. A. Kelly, R. L. Bramblett, S. C. Fultz, Phys. Rev. 162 (1967) 1098.

[1968Be5] R. Bergere, R. Beil, A. Veyssiere, Nucl. Phys. A121 (1968) 463.

[1968Bez] N. Bezic, D. Jamnik, G. Kernel, J. Krajnik, J. Snajde, Nucl. Phys. A117 (1968) 124.

[1968Dol] B. S. Dolbilkin, A. I. Isakov, V. I. Korin, L. E. Lazareva, F. A. Nikolaev, Yad. Fiz. 8 (1968) 1080. ( Soviet J. Nucl. Phys. 8 (1969) 626.)

[1968Su1] R. E. Sund, M. P. Baker, L. A. Kull, R. B. Walton, Phys. Rev. 176 (1968) 1366.

[1968Tom] T. Tomimasu, J. Phys. Soc. Japan 25 (1968) 655. 
[1969Be1] B. L. Berman, R. L. Bramblett, J. T. Caldwell, H. S. Davis, M. A. Kelly, S. C. Fultz, Phys. Rev. 177 (1969) 1745 .

[1969Be6] R. Bergere, H. Beil, P. Carlos, A. Veyssiere, Nucl. Phys. A133 (1969) 417.

[1969Be8] B. L. Berman, M. A. Kelly, R. L. Bramblett, J. T. Caldwell, H. S. Davis, S. C. Fultz, Phys. Rev. 185 (1969) 1576.

[1969Bez] N. Bezic, D. Brajnik, D. Jamnik, G. Kernel, Nucl. Phys. A128 (1969) 426.

[1969Dea] T. K. Deague, E. G. Muirhead, B. M. Spicer, Nucl. Phys. A139 (1969) 501.

[1969Fu1] S. C. Fultz, B. L. Berman, J. T. Caldwell, R. L. Bramblett, M. A. Kelly, Phys. Rev. 186 (1969) 1255.

[1969Gor] B. I. Goryachev, B. S. Ishkhanov, I. M. Kapitonov, I. M. Piskarev, V. G. Shevchenko, O. P. Shevchenko, Izv. Akad. Nauk SSSR, Ser. Fiz. 33 (1969) 1736 (Bull. Acad. Sci. USSR, Phys. Ser. 33 (1970) 1588).

[1969Ish] B. S. Ishkhanov, I. M. Kapitonov, E. V. Lazutin, I. M. Piskarev, O. P. Shevchenko, ZhETF Pisma v Redaktsiyu 10 (1969) 30 (JETP Letters (USSR) 10 (1969) 51).

[1969Vas] O. V. Vasilev, G. N. Zalesnyi, S. F. Semenko, V. A. Semenov, Yad. Fiz. 10 (1969) 460 (Soviet J. Nucl. Phys. 10 (1969) 263).

[1970Ant] G. P. Antropov, I. E. Mitrofanov, A. I. Prokofev, V. S. Russkikh Izv. Akad. Nauk SSSR, Ser. Fiz 34 (1970) 116 (Bull. Acad. Sci. USSR, Phys. Ser. 34 (1970) 108).

[1970Be8] B. L. Berman, S. C. Fultz, J. T. Caldwell, M. A. Kelly, S. S. Dietrich, Phys. Rev. C2 (1970) 2318.

[1970Gor] B. I. Goryachev, B. S. Ishkhanov, I. M. Kapitonov, I. M. Piskarev, V. G. Shevchenko, O. P. Shevchenko; Yad. Fiz. 11 (1970) 252 (Sov. J. Nucl. Phys. 11 (1970) 141).

[1970Is1] B. S. Ishkhanov, I. M. Kapitonov, E. V. Lazutin, I. M. Piskarev, O. P. Shevchenko, Izv. Akad. Nauk SSSR, Ser. Fiz. 34 (1970) 2228 (Bull. Acad. Sci. USSR, Phys. Ser. 34 (1971) 1988).

[1970Is2] B. S. Ishkhanov, I. M. Kapitonov, E. V. Lazutin, I. M. Piskarev, O. P. Shevchenko, Vestnik Moskovskogo Univ., Ser. Fiz. Astron. 6 (1970) 606.

[1970Is3] B. S. Ishkhanov, I. M. Kapitonov, E. V. Lazutin, I. M. Piskarev, O. P. Shevchenko, Yad. Fiz. 11 (1970) 606 (Sov. J. Nucl. Phys. 11 (1970) 394).

[1970Su1] R. E. Sund, V. V. Verbinski, H. Weber, L. A. Kull, Phys. Rev. C2 (1970) 1129.

[1970Ve1] A. Veyssiere, H. Beil, R. Bergere, P. Carlos, A. Lepretre, Nucl. Phys. A159 (1970) 561.

[1971Alv] R. A. Alvarez, B. L. Berman, D. R. Lasher, T. W. Phillips, S. C. Fultz, Phys. Rev. C4 (1971) 1673.

[1971Be4] H. Beil, R. Bergere, P. Carlos, A. Lepretre, A. Veyssiere, A. Parlag, Nucl. Phys. A172 (1971) 426.

[1971Ca1] P. Carlos, H. Beil, R. Bergere, A. Lepretre, A. Veyssiere, Nucl. Phys. A172 (1971) 437.

[1971Fu2] S. C. Fultz, R. A. Alvarez, B. L. Berman, M. A. Kelly, D. R. Lasher, T. W. Phillips, J. McElhinney, Phys. Rev. C4 (1971) 149 .

[1971Le1] A. Lepretre, H. Beil, R. Bergere, P. Carlos, A. Veyssiere, M. Sugawara, Nucl. Phys. A175 (1971) 609. 
[1971Vas] O. V. Vasilev, V. A. Semenov, S. F. Semenko, Yad. Fiz. 13 (1971) 463 (Sov. J. Nucl. Phys. 13 (1971) 259).

[1972Ahr] J. Ahrens, H. Borchert, H. B. Eppler, H. Gimm, H. Gundrum, Conf.Nucl.Structure Studies, Sendai, Japan, (1972) 213 .

[1972Ask] H. J. Askin, R. S. Hicks, K. J. F. Allen, R. J. Petty, M. N. Thompson Nucl. Phys. A204 (1973) 209.

[1972De1] T. K. Deague, R. J. Stewart, Nucl. Phys. A91 (1972) 305.

[1972You] L. M. Young, Ph.D. Thesis, University of Illinois(1972), unpublished.

[1973Bra] R. L. Bramblett, B. L. Berman, M. A. Kelly, J. T. Caldwell, S. C. Fultz, Int. Conf. on Photonucl. Reactions, Pacific Grove 1 (1973) 175.

[1973Gor] A. M. Goryachev, G. N. Zalesnyi, S. F. Semenko, B. A. Tulupov, Yad. Fiz. 17 (1973) 463 (Soviet J. Nucl. Phys. 17 (1974) 236.).

[1973Sor] Y. I. Sorokin, V. A. Khrushchev, B. A. Yurev Izv. Akad. Nauk SSSR, Ser. Fiz. 37(9) (1973) 1890 (Bull. Acad. Sci. USSR, Phys. Ser. 37(9) (1974) 80).

[1973Ve1] A. Veyssiere, H. Reil, R. Bergere, P. Carlos, A. Lepretre, K. Kernbach, Nucl. Phys. A199 (1973) 45.

[1974Be3] H. Beil, R. Bergere, P. Carlos, A. Lepretre, A. De Miniac, A. Veyssiere, Nucl. Phys. A227 (1974) 427.

[1974Ca5] P. Carlos, H. Beil, R. Bergere, A. Lepretre, A. De Miniac, A. Veyssiere, Nucl. Phys. A225 (1974) 171.

[1974Car] R. Carchon, J. Devos, R. Van de Vyver, C. Van Deynse, H. Ferdinande, Nucl. Phys. A223 (1974) 416.

[1974Fu3] S. C. Fultz, R. A. Alvarez, B. L. Berman, P. Meyer, Phys. Rev. C10 (1974) 608.

[1974Le1] A. Lepretre, H. Beil, R. Bergere, P. Carlos, A. De Miniac, A. Veyssiere, K. Kernbach, Nucl. Phys. A219 (1974) 39 .

[1974Sor] Y. I. Sorokin, B. A. Yurev, Yad. Fiz. 20 (1974) 233 (Soviet J. Nucl. Phys. 20 (1975) 123).

[1974Ve1] A. Veyssiere, H. Beil, R. Bergere, P. Carlos, A. Lepretre, A. De Miniac, Nucl. Phys. A227 (1974) 513.

[1975Ahr] J. Ahrens, H. Borchert, K. H. Czock, H. B. Eppler, H. Gimm, H. Gundrum, M. Kroning, P. Riehn, G. Sita Ram, A. Zieger, B. Ziegler, Nucl. Phys. A251 (1975) 479.

[1975Mcc] J. J. McCarthy, R. C. Morrison, H. J. Vander-Molen, Phys. Rev. C11 (1975) 772.

[1975Sor] Y. I. Sorokin, B. A. Yurev Izv. Akad. Nauk SSSR, Ser. Fiz. 39 (1975) 114 (Bull. Acad. Sci. USSR, Phys. Ser. 39(1) (1975) 98).

[1975Vey] A. Veyssiere, H. Beil, R. Bergere, P. Carlos, A. Lepretre, A. De Miniac, Jour. de Phys. Lett. 36 (1975) 267.

[1976Ca1] P. Carlos, H. Beil, R. Bergere, J. Fagot, A. Lepretre, A. Veyssiere, G. V. Solodukhov, Nucl. Phys. A258 (1976) 365 .

[1976Gor] B. I. Goryachev, Y. V. Kuznetsov, V. N. Orlin, N. A. Pozhidaeva, V. G. Shevchenko, Yad. Fiz. 23 (1976) 1145.

[1976Gu1] G. M. Gurevich, L. E. Lazareva, V. M. Mazur, G. V. Solodukhov, B. A. Tulupov, Nucl. Phys. A273 (1976) 326 . 
[1976Gu2] G. M. Gurevich, L. E. Lazareva, V. M. Mazur, G. V. Solodukhov, JETP Lett.(USSR) 23 (1976) 370.

[1976Le2] A. Lepretre, H. Beil, R. Bergere, P. Carlos, J. Fagot, A. De Miniac, A. Veyssiere, H. Miyase, Nucl. Phys. A258 (1976) 350.

[1977Gor] A. M. Goryachev, G. N. Zalesnyi, Yad. Fiz. 26 (1977) 465 (Sov. J. Nucl. Phys. 26 (1977) 246).

[1978Go1] A. M. Goryachev, G. N. Zalesnyi, JETP Letters. 26 (1978) 99 (Zhur. Eksp. Teor. Fiz., Pisma v Redakt. 26 (1978) 107).

[1978Go2] A. M. Goryachev, G. N. Zalesnyi, Yad. Fiz. 27 (1978) 1479 (Sov. J. Nucl. Phys. 27 (1978) 779).

[1978Go3] A. M. Goryachev, G. N. Zalesnyi, Izvestiya Akademii Nauk KazSSSR, Ser. Fiz. -Mat. 6 (1978) 8.

[1978Gur] G. M. Gurevich, L. E. Lazareva, V. M. Mazur, G. V. Solodukhov, Prob. Yad. Fiz. Kosm. Luch. 8 (1978) 106.

[1978Nor] J. W. Norbury, M. N. Thompson, K. Shoda, H. Tsubota, Australian Jour. of Phys. 31 (1978) 471.

[1979A12] R. A. Alvarez, B. L. Berman, D. D. Faul, F. H. Lewis, Jr., P. Meyer, Phys. Rev. C20 (1979) 128.

[1979Be4] B. L. Berman, D. D. Faul, R. A. Alvarez, P. Meyer, D. L. Olson, Phys. Rev. C19 (1979) 1205.

[1980Ca1] J. T. Caldwell, E. J. Dowdy, B. L. Berman, R. A. Avarez, P. Meyer, Phys. Rev. C21 (1980) 1215.

[1981Gur] G. M. Gurevich, L. E. Lazareva, V. M. Mazur, S. Yu. Merkulov, G. V. Solodukhov, V. A. Tyutin, Nucl. Phys. A351 (1981) 257.

[1981Ish] B. S. Ishkhanov, I. M. Kapitonov, V. I. Shvedunov, A. I. Gutii, A. M. Parlag, Yad. Fiz. 33 (1981) 581 (Sov. J. Nucl. Phys. 33 (1981) 303).

[1985Ahr] J. Ahrens, Nucl. Phys. A446 (1985) 229.

[1986Ass] Y. I. Assafiri, M. N. Thompson, Nucl. Phys. A460 (1986) 455.

[1986Be2] B. L. Berman, J. T. Caldwell, E. J. Dowdy, S. S. Dietrich, P. Meyer, R. A. Alvarez, Phys. Rev. C34 (1986) 2201.

[1986Var] V. V. Varlamov, V. V. Surgutanov, A. P. Chernyaev, N. G. Efimkin, Book: Fotojad. Dannye - Photodisint. of Li, Suppl., Moscow (1986).

[1987Ahs] M. H. Ahsan, S. A. Siddiqui, H. H. Thies, Nucl. Phys. A469 (1987) 381.

[1987Ber] B. L. Berman, R. G. Pywell, S. S. Dietrich, M. N. Thompson, K. O. McNeill, J. W. Jury, Phys. Rev. C36 (1987) 1286.

[1987OKe] G. J. O'Keefe, M. N. Thompson, Y. I. Assafiri, R. E. Pywel, Nucl. Phys. A469 (1987) 239.

[1989Ras] R. P. Rassool, M. N. Thompson, Phys. Rev. C39 (1989) 1631; Phys. Rev. C40 (1989) 506.

[1995Bel] S. N. Belyaev, O. V. Vasiliev, V. V. Voronov, A. A. Nechkin, V. Yu. Ponomarev, V. A. Semenov, Yad. Fiz. 58 (1995) 1940 (Phys. Atomic Nuclei 58 (1995) 1883).

[1999Bel] S. N. Belyaev, V. A. Semenov, V. P. Sinichkin, Workshop on Beam Dynamics and Optimiz, Saratov (1997) 97.

[2001Bel] S. N. Belyaev, V. P. Sinichkin, Conf.: Workshop on Beam Dynamics and Optimiz, Saratov (2001) 81. 
[2002Ish] B. S. Ishkhanov, I. M. Kapitonov, E. I. Lileeva, E. V. Shirokov, V. A. Erokhova, M. A. Elkin, A. V. Izotova, Moscow State Univ. Inst. of Nucl. Phys. Report No.2002 27/711 (2002).

[2003Ero] V. A. Erokhova, M. A. Elkin, A. V. Izotova, B. S. Ishkhanov, I. M. Kapitonov, E. I. Lilieva, E. V. Shirokov, Izv. Ros. Akad. Nauk, Ser. Fiz. 67 (2003) 1479.

[2003Rod] T. E. Rodrigues, J. D. T. Arruda-Neto, Z. Carvaheiro, J. Mesa, Phys. Rev. C68 (2003) 68.

[2003Var] V. V. Varlamov, M. E. Stepanov, V. V. Chesnokov, Izv. Ros. Akad. Nauk, Ser. Fiz. 67 (2003) 656 (Bull. Rus. Acad. Sci. Phys. 67 (2003) 724).

[2006Var] V. V. Varlamov, B. S. Ishkhanov, I. V. Makarenko, V. N. Orlin, N. N. Peskov, Moscow State Univ. Inst. of Nucl. Phys. Reports, 9 (2006) 808.

[2007Var] V. V. Varlamov, N. N. Peskov; Moscow State Univ. Inst. of Nucl. Phys. Reports, 8 (2007) 829.

[2009Var] V. V. Varlamov,B. S. Ishkhanov, V. N. Orlin, V. A. Tchetvertkova, Moscow State Univ. Inst. of Nucl. Phys. Reports, 3 (2009) 847 In Russian., Izv. Rossiiskoi Akademii Nauk, Ser. Fiz 74 (2010) 875 (Bull. Rus. Acad. Sci. Phys. 74 (2010) 833).

[2010Var] V. V. Varlamov, B. S. Ishkhanov, V. N. Orlin, V. A. Tchetvertkova, Izv. Ros. Akad. Nauk, Ser. Fiz. 74 (2010) 884 (Bull. Rus. Acad. Sci. Phys. 74 (2010) 842).

[2011Var] V. V. Varlamov, B. S. Ishkhanov, V. N. Orlin, T. S. Polevich, M. E. Stepanov, Moscow State Univ. Inst. of Nucl. Phys. Reports, 5 (2011) 869 In Russian. ; Yad. Fiz. 75(11) (2012) 1414 (Phys. At. Nucl. 75(11) (2012) 1339), Eur. Phys. J. A 50 (2014) 114.

[2012Var] V. V. Varlamov, V. N. Orlin, N. N. Peskov, T. S. Polevich; Moscow State Univ. Inst. of Nucl. Phys. Reports, 1 (2012) 879 In Russian., Yad. Fiz. 76(11) (2013) 1484 (Phys. At. Nucl. 76(11) (2013) 1403), Yad. Fiz. 77(12) (2014) 1563 (Phys. At. Nucl. 77(12) (2014) 1369).

[2013Ish] B. S. Ishkhanov, V. N. Orlin, N. N. Peskov, M. E. Stepanov, V. V. Varlamov, Moscow State Univ. Inst. of Nucl. Phys. Reports, 1 (2013) 884 In Russian., Izv. Akad. Nauk RAS, Ser. Fiz 77 (2013) 433 (Bull. Rus. Acad. Sci. Phys. 77 (2013) 388).

[2013Var] V. V. Varlamov, V. N. Orlin, N. N. Peskov, M. E. Stepanov; Izv. Ros. Akad. Nauk, Ser. Fiz. 77 (2013) 433 (Bull. Rus. Acad. Sci. Phys. 77 (2013) 388).

[2014Var] V. V. Varlamov, M. A. Makarov, N. N. Peskov, M. E. Stepanov; Izv. Ros. Akad. Nauk, Ser. Fiz. 78 (2014) 599 (Bull. Russ. Acad. Sci. Phys. 78(5) (2014) 412).

[2015Var] V. V. Varlamov, M. A. Makarov, N. N. Peskov, M. E. Stepanov, Yad. Fiz. 78 (2015) 678.

[2016Va1] V. V. Varlamov, A. I. Davydov,M. A. Makarov,V. N. Orlin, N. N. Peskov, Izv. Ros. Akad. Nauk, Ser. Fiz. 80 (2016) 351 (Bull. Rus. Acad. Sci. Phys. 80 (2016) 317).

[2016Va2] V. V. Varlamov, B. S. Ishkhanov, V. N. Orlin, N. N. Peskov, Yad. Fiz. 79 (2016) 315 (Journ. Phys. Atom. Nuclei $79(4)$ (2016) 501). 
Table 1

Experimental values and uncertainties of GDR parameters within Standard Lorentzian (SLO) approach.

\begin{tabular}{|c|c|c|c|c|c|c|c|c|c|c|c|c|c|c|c|c|c|}
\hline \multirow{2}{*}{$\begin{array}{l}\text { Nucl } \\
{ }^{6} \mathrm{Li}\end{array}$} & \multirow{2}{*}{$\begin{array}{l}\text { Id } \\
2\end{array}$} & \multicolumn{2}{|c|}{$\begin{array}{c}E_{r, 1} \\
(\mathrm{MeV})\end{array}$} & \multicolumn{2}{|c|}{$\begin{array}{c}\Gamma_{r, 1} \\
(\mathrm{MeV})\end{array}$} & \multicolumn{2}{|l|}{$s_{1}$} & \multicolumn{2}{|c|}{$\begin{array}{c}E_{r, 2} \\
(\mathrm{MeV}) \\
\end{array}$} & \multicolumn{2}{|c|}{$\begin{array}{c}\Gamma_{r, 2} \\
(\mathrm{MeV}) \\
\end{array}$} & $s_{2}$ & & $s$ & & $\begin{array}{c}\varepsilon_{\min }-\varepsilon_{\max } \\
(\mathrm{MeV})\end{array}$ & Ref \\
\hline & & 23.69 & 16 & 5.26 & 79 & 0.341 & 31 & & & & & & & 0.341 & 31 & $21.5-27.0$ & 1986Var \\
\hline & 4 & 12.18 & 5 & 11.58 & 1 & 0.334 & 150 & & & & & & & 0.334 & 4 & $5.6-24.7$ & 1965Be1 \\
\hline${ }^{7} \mathrm{Li}$ & 0 & 18.59 & 21 & 16.28 & 116 & 0.907 & 51 & & & & & & & 0.907 & 51 & $13.2-25.6$ & 1985Ahr \\
\hline & $2 \mathrm{a}$ & 16.39 & 11 & 16.03 & 42 & 0.858 & 22 & & & & & & & 0.858 & 22 & $13.5-25.0$ & 1986Var \\
\hline & $2 \mathrm{~b}$ & 16.40 & 11 & 16.03 & 42 & 0.858 & 22 & & & & & & & 0.858 & 22 & $13.5-25.0$ & 1986Var \\
\hline & $4 a$ & 16.34 & 7 & 10.42 & 24 & 0.236 & 4 & & & & & & & 0.236 & 4 & $10.1-24.7$ & 1973Bra \\
\hline & $4 \mathrm{~b}$ & 16.26 & 10 & 11.17 & 41 & 0.248 & 7 & & & & & & & 0.248 & 7 & $10.1-24.7$ & 1973Bra \\
\hline${ }^{9} \mathrm{Be}$ & 0 & 23.75 & 10 & 9.47 & 33 & 0.577 & 17 & & & & & & & 0.577 & 17 & $17.5-26.0$ & 1975Ahr \\
\hline${ }^{10} \mathrm{~B}$ & $4 a$ & 21.72 & 10 & 9.08 & 16 & 0.441 & 8 & & & & & & & 0.441 & 8 & $8.5-24.9$ & 1987Ahs \\
\hline & $4 \mathrm{~b}$ & 22.54 & 32 & 10.81 & 63 & 0.518 & 26 & & & & & & & 0.518 & 26 & $8.5-24.9$ & 1987Ahs \\
\hline${ }^{12} \mathrm{C}$ & 0 & 22.86 & 2 & 3.61 & 7 & 0.671 & 10 & & & & & & & 0.671 & 10 & $20.1-25.0$ & 1969Bez \\
\hline & 1 & 22.88 & 2 & 3.06 & & 0.573 & 70 & & & & & & & 0.573 & 10 & $13.2-24.0$ & 1975Ahr \\
\hline & $3 a$ & 23.14 & 5 & 2.89 & 11 & 0.546 & 13 & & & & & & & 0.546 & 13 & $20.2-25.0$ & 2002Ish \\
\hline & $3 \mathrm{~b}$ & 23.09 & 7 & 2.95 & 15 & 0.549 & 21 & & & & & & & 0.549 & 21 & $20.2-25.0$ & 2002Ish \\
\hline & 4 & 22.79 & 7 & 3.62 & 28 & 0.494 & 27 & & & & & & & 0.494 & 27 & $14.0-24.9$ & 1963Bur \\
\hline${ }^{13} \mathrm{C}$ & $3 a$ & 24.60 & 13 & 8.43 & 41 & 0.868 & 22 & & & & & & & 0.868 & 22 & $14.5-29.0$ & 2002Ish \\
\hline & $3 \mathrm{~b}$ & 24.40 & 17 & 7.70 & 63 & 0.828 & 36 & & & & & & & 0.828 & 36 & $14.5-29.0$ & 2002Ish \\
\hline${ }^{14} \mathrm{C}$ & $3 a$ & 15.41 & 26 & 5.82 & 90 & 0.333 & 47 & 26.13 & 15 & 7.78 & 92 & 0.483 & 46 & 0.816 & 66 & $14.5-30.0$ & 2002Ish \\
\hline & $3 b$ & 16.68 & 57 & 3.10 & 67 & 0.177 & 23 & 25.87 & 17 & 6.84 & 84 & 0.490 & 46 & 0.667 & 51 & $9.0-30.0$ & 2002Ish \\
\hline nat $\mathrm{C}$ & 0 & 23.12 & 2 & 4.19 & 6 & 0.705 & 7 & & & & & & & 0.705 & 7 & $19.5-25.6$ & 1985Ahr \\
\hline & 0 & 23.38 & 1 & 4.17 & 4 & 0.728 & 5 & & & & & & & 0.728 & 5 & $19.0-26.0$ & 1975Ahr \\
\hline & 0 & 23.14 & 2 & 4.03 & 7 & 0.678 & 8 & & & & & & & 0.678 & 8 & $19.1-25.8$ & 1972Ahr \\
\hline & $1 \mathrm{a}$ & 23.61 & 11 & 2.56 & 17 & 0.442 & 13 & & & & & & & 0.442 & 13 & $19.1-26.0$ & $1965 \mathrm{Wyc}$ \\
\hline & $1 b$ & 24.04 & 20 & 3.40 & 36 & 0.480 & 27 & & & & & & & 0.480 & 27 & $19.1-26.0$ & $1965 \mathrm{Wyc}$ \\
\hline${ }^{14} \mathrm{~N}$ & 0 & 23.05 & 3 & 6.95 & 13 & 1.193 & 16 & & & & & & & 1.193 & 16 & $18.2-28.0$ & 1969Bez \\
\hline & $3 a$ & 23.39 & 8 & 4.83 & 17 & 0.729 & 15 & & & & & & & 0.729 & 15 & $15.0-28.0$ & 2002Ish \\
\hline & $3 \mathrm{~b}$ & 23.13 & 18 & 4.83 & 35 & 0.725 & 47 & & & & & & & 0.725 & 47 & $15.0-28.0$ & 2002Ish \\
\hline${ }^{15} \mathrm{~N}$ & $3 a$ & 24.78 & 26 & 12.82 & 63 & 1.242 & 56 & & & & & & & 1.242 & 56 & $14.5-28.0$ & 2002Ish \\
\hline & $3 \mathrm{~b}$ & 24.96 & 39 & 12.47 & 134 & 1.259 & 111 & & & & & & & 1.259 & 111 & $14.5-28.0$ & 2002Ish \\
\hline${ }^{16} \mathrm{O}$ & 0 & 23.70 & 4 & 5.36 & 12 & 0.981 & 16 & & & & & & & 0.981 & 16 & $18.1-26.0$ & $1975 \mathrm{Ahr}$ \\
\hline & 0 & 23.66 & 4 & 5.44 & 12 & 0.985 & 16 & & & & & & & 0.985 & 16 & $18.2-25.9$ & 1972Ahr \\
\hline & 0 & 23.37 & 4 & 5.54 & 14 & 0.957 & 19 & & & & & & & 0.957 & 19 & $18.5-26.0$ & 1969Bez \\
\hline & 0 & 22.92 & 5 & 4.73 & 17 & 1.021 & 28 & & & & & & & 1.021 & 28 & $18.9-25.9$ & 1963Bur \\
\hline & $1 \mathrm{a}$ & 23.92 & 10 & 2.58 & 11 & 0.444 & 15 & & & & & & & 0.444 & 15 & $18.2-25.6$ & $1965 \mathrm{Wyc}$ \\
\hline & $1 b$ & 24.04 & 13 & 2.90 & 18 & 0.469 & 22 & & & & & & & 0.469 & 22 & $18.2-25.6$ & $1965 \mathrm{Wyc}$ \\
\hline & $3 a$ & 23.71 & 7 & 3.98 & 12 & 0.762 & 17 & & & & & & & 0.762 & 17 & $18.2-26.0$ & 2002Ish \\
\hline & $3 \mathrm{~b}$ & 23.89 & 11 & 4.59 & 23 & 0.820 & 29 & & & & & & & 0.820 & 29 & $18.2-26.0$ & 2002Ish \\
\hline${ }^{17} \mathrm{O}$ & $3 a$ & 23.40 & 10 & 5.48 & 31 & 0.739 & 24 & & & & & & & 0.739 & 24 & $18.5-26.5$ & 2002Ish \\
\hline & $3 \mathrm{~b}$ & 23.38 & 13 & 5.57 & 49 & 0.740 & 36 & & & & & & & 0.740 & 36 & $18.5-26.5$ & 2002Ish \\
\hline${ }^{18} \mathrm{O}$ & $3 a$ & 19.08 & 14 & 2.12 & 57 & 0.064 & 18 & 24.10 & 16 & 5.25 & 83 & 0.417 & 49 & 0.481 & 52 & $18.5-26.0$ & 2002Ish \\
\hline nat $\mathrm{O}$ & 0 & 23.60 & 4 & 5.82 & 12 & 1.031 & 15 & & & & & & & 1.031 & 15 & $18.9-27.9$ & 1985Ahr \\
\hline & 0 & 23.84 & 3 & 6.08 & 9 & 1.101 & 12 & & & & & & & 1.101 & 12 & $18.6-27.8$ & 1975Ahr \\
\hline${ }^{19} \mathrm{~F}$ & $3 a$ & 21.61 & 36 & 12.57 & 7 & 1.152 & 580 & & & & & & & 1.152 & 67 & $10.0-24.0$ & 2002Ish \\
\hline${ }^{23} \mathrm{Na}$ & 0 & 17.43 & 13 & 3.10 & 42 & 0.175 & 35 & 21.13 & 10 & 4.51 & 52 & 0.555 & 63 & 0.730 & 72 & $14.2-23.0$ & 1981Ish \\
\hline & $1 \mathrm{a}$ & 19.56 & 27 & 6.99 & 65 & 0.516 & 50 & 24.77 & 14 & 2.81 & 85 & 0.135 & 41 & 0.650 & 65 & $14.5-25.9$ & $1965 \mathrm{Wyc}$ \\
\hline & $1 \mathrm{~b}$ & 19.76 & 30 & 7.55 & 90 & 0.549 & 63 & 24.80 & 13 & 2.45 & 77 & 0.112 & 37 & 0.661 & 73 & $14.5-25.9$ & $1965 \mathrm{Wyc}$ \\
\hline & $3 a$ & 17.45 & 15 & 3.08 & 33 & 0.178 & 34 & 20.98 & 12 & 4.34 & 49 & 0.492 & 55 & 0.670 & 65 & $14.3-23.0$ & 2002Ish \\
\hline & $3 b$ & 17.39 & 22 & 2.90 & 69 & 0.161 & 48 & 20.94 & 14 & 4.55 & 59 & 0.519 & 71 & 0.680 & 86 & $14.3-23.0$ & 2002 Ish \\
\hline & 4 & 17.88 & 9 & 4.59 & 21 & 0.151 & 11 & 24.34 & 20 & 9.22 & 55 & 0.368 & 25 & 0.518 & 27 & $12.6-25.7$ & $1971 \mathrm{Alv}$ \\
\hline${ }^{24} \mathrm{Mg}$ & 0 & 19.51 & 12 & 2.71 & 40 & 0.253 & 58 & 23.88 & 36 & 8.86 & 144 & 0.974 & 163 & 1.227 & 173 & $16.5-27.0$ & 1966Dol \\
\hline & $3 a$ & 19.81 & 5 & 2.72 & 12 & 0.359 & 17 & 24.42 & 9 & 4.76 & 34 & 0.416 & 27 & 0.775 & 32 & $16.3-27.0$ & 2003Var \\
\hline & $3 \mathrm{~b}$ & 19.79 & 6 & 2.98 & 18 & 0.383 & 24 & 24.54 & 9 & 4.85 & 44 & 0.415 & 36 & 0.798 & 43 & $16.3-27.0$ & 2003Var \\
\hline & $3 a$ & 19.88 & 7 & 2.81 & 18 & 0.371 & 26 & 24.69 & 11 & 4.74 & 58 & 0.451 & 46 & 0.822 & 53 & $15.2-27.0$ & 2002Ish \\
\hline & $3 b$ & 19.85 & 7 & 2.79 & 19 & 0.373 & 25 & 24.71 & 10 & 4.69 & 53 & 0.451 & 44 & 0.824 & 50 & $15.2-27.0$ & 2002Ish \\
\hline${ }^{25} \mathrm{Mg}$ & $3 a$ & 22.06 & 10 & 6.09 & 15 & 0.894 & 20 & & & & & & & 0.894 & 20 & $9.0-24.2$ & 2002Ish \\
\hline & $3 \mathrm{~b}$ & 22.09 & 16 & 6.30 & 30 & 0.902 & 34 & & & & & & & 0.902 & 34 & $9.0-24.2$ & 2002Ish \\
\hline & 4 & 23.26 & 22 & 9.56 & 44 & 0.947 & 52 & & & & & & & 0.947 & 52 & $14.1-23.9$ & 1971Alv \\
\hline${ }^{26} \mathrm{Mg}$ & $3 \mathrm{a}$ & 17.38 & 5 & 2.15 & 17 & 0.144 & 11 & 23.64 & 8 & 7.25 & 31 & 1.149 & 38 & 1.294 & 39 & $16.1-26.5$ & 2003Var \\
\hline & $3 \mathrm{~b}$ & 17.37 & 12 & 1.68 & 29 & 0.113 & 17 & 23.58 & 8 & 7.75 & 37 & 1.222 & 45 & 1.335 & 48 & $16.1-26.5$ & 2003Var \\
\hline & $3 a$ & 17.48 & 11 & 1.89 & 33 & 0.117 & 19 & 23.65 & 18 & 6.93 & 64 & 1.023 & 74 & 1.140 & 77 & $16.4-26.0$ & 2002Ish \\
\hline & $3 \mathrm{~b}$ & 17.50 & 24 & 1.41 & 50 & 0.088 & 27 & 23.61 & 19 & 7.47 & 76 & 1.095 & 89 & 1.183 & 93 & $16.4-26.0$ & 2002Ish \\
\hline & 4 & 17.42 & 3 & 2.53 & 11 & 0.164 & 7 & 22.86 & 12 & 5.05 & 29 & 0.428 & 27 & 0.592 & 27 & $16.0-25.7$ & 1971Fu2 \\
\hline${ }^{\text {nat }} \mathrm{Mg}$ & $1 \mathrm{a}$ & 22.55 & 21 & 7.97 & 37 & 0.726 & 26 & & & & & & & 0.726 & 26 & $15.1-26.6$ & $1965 \mathrm{Wyc}$ \\
\hline & $1 b$ & 20.08 & 16 & 4.04 & 36 & 0.499 & 31 & & & & & & & 0.499 & 31 & $15.1-26.6$ & $1965 \mathrm{Wyc}$ \\
\hline $27 \mathrm{Al}$ & 0 & 20.73 & 8 & 7.45 & 26 & 1.128 & 30 & & & & & & & 1.128 & 30 & $14.0-24.1$ & 1985Ahr \\
\hline
\end{tabular}


Table 1 (continued)

\begin{tabular}{|c|c|c|c|c|c|c|c|c|c|c|c|c|c|c|c|c|c|}
\hline \multirow{2}{*}{$\begin{array}{l}\text { Nucl } \\
\end{array}$} & \multirow{2}{*}{$\frac{\text { Id }}{0}$} & \multicolumn{2}{|c|}{$\begin{array}{c}E_{r, 1} \\
(\mathrm{MeV})\end{array}$} & \multicolumn{2}{|c|}{$\begin{array}{c}\Gamma_{r, 1} \\
(\mathrm{MeV})\end{array}$} & \multicolumn{2}{|l|}{$s_{1}$} & \multicolumn{2}{|c|}{$\begin{array}{c}E_{r, 2} \\
(\mathrm{MeV})\end{array}$} & $\begin{array}{r}\Gamma_{r, 2} \\
(\mathrm{MeV} \\
\end{array}$ & & $s_{2}$ & & $s$ & & $\begin{array}{c}\varepsilon_{\min }-\varepsilon_{\max } \\
(\mathrm{MeV})\end{array}$ & Ref \\
\hline & & 20.71 & 6 & 7.57 & 20 & 1.138 & 23 & & & & & & & 1.138 & 23 & $14.2-24.4$ & 1975Ahr \\
\hline & 0 & 20.85 & 6 & 7.19 & 22 & 1.078 & 24 & & & & & & & 1.078 & 24 & $14.2-24.4$ & 1972Ahr \\
\hline & 0 & 20.84 & 6 & 7.50 & 19 & 1.122 & 23 & & & & & & & 1.122 & 23 & $14.2-24.4$ & 1975Ahr \\
\hline & $3 a$ & 20.58 & 7 & 4.46 & 11 & 0.772 & 16 & & & & & & & 0.772 & 16 & $14.2-23.0$ & 2002Ish \\
\hline & 4 & 20.77 & 2 & 5.96 & 4 & 0.328 & 2 & & & & & & & 0.328 & 2 & $14.0-24.4$ & 1966Fu1 \\
\hline${ }^{28} \mathrm{Si}$ & $3 a$ & 19.81 & 12 & 2.56 & 20 & 0.371 & 73 & 21.81 & 16 & 3.15 & 36 & 0.474 & 89 & 0.845 & 115 & $16.7-23.0$ & 2003Var \\
\hline & $3 \mathrm{~b}$ & 19.73 & 21 & 2.24 & 86 & 0.238 & 183 & 21.56 & 36 & 4.03 & 68 & 0.676 & 236 & 0.914 & 299 & $16.7-23.0$ & 2003Var \\
\hline & $3 a$ & 18.84 & 13 & 1.57 & 19 & 0.152 & 32 & 20.95 & 10 & 3.01 & 19 & 0.515 & 41 & 0.667 & 52 & $16.8-23.0$ & 2002Ish \\
\hline & $3 \mathrm{~b}$ & 19.05 & 32 & 1.82 & 57 & 0.158 & 86 & 20.93 & 22 & 3.48 & 30 & 0.548 & 100 & 0.706 & 131 & $16.8-23.0$ & 2002Ish \\
\hline${ }^{29} \mathrm{Si}$ & $3 a$ & 20.70 & 8 & 5.60 & 17 & 0.810 & 19 & & & & & & & 0.810 & 19 & $14.2-23.0$ & 2002Ish \\
\hline & $3 \mathrm{~b}$ & 20.73 & 12 & 5.76 & 34 & 0.821 & 32 & & & & & & & 0.821 & 32 & $14.2-23.0$ & 2002Ish \\
\hline${ }^{30} \mathrm{Si}$ & $3 a$ & 20.86 & 13 & 7.40 & 31 & 0.767 & 27 & & & & & & & 0.767 & 27 & $14.2-23.0$ & 2002Ish \\
\hline & $3 \mathrm{~b}$ & 20.91 & 19 & 7.51 & 58 & 0.778 & 48 & & & & & & & 0.778 & 48 & $14.2-23.0$ & 2002Ish \\
\hline${ }^{\text {nat }} \mathrm{Si}$ & 0 & 20.35 & 3 & 4.53 & 9 & 0.871 & 18 & 25.16 & 19 & 2.86 & 67 & 0.112 & 25 & 0.983 & 31 & $16.4-25.8$ & $1975 \mathrm{Ahr}$ \\
\hline${ }^{32} \mathrm{~S}$ & 0 & 19.51 & 8 & 4.83 & 31 & 0.560 & 25 & & & & & & & 0.560 & 25 & $14.7-23.0$ & 1968Dol \\
\hline & $3 a$ & 21.17 & 9 & 5.08 & 12 & 0.967 & 25 & & & & & & & 0.967 & 25 & $14.4-23.0$ & 2002Ish \\
\hline & $3 b$ & 21.51 & 14 & 5.67 & 30 & 1.082 & 50 & & & & & & & 1.082 & 50 & $14.4-23.0$ & 2002Ish \\
\hline${ }^{34} \mathrm{~S}$ & 0 & 20.89 & 47 & 9.61 & 77 & 1.501 & 149 & & & & & & & 1.501 & 149 & $12.0-25.0$ & 1986 Ass \\
\hline & $3 a$ & 21.13 & 10 & 12.58 & 36 & 1.755 & 41 & & & & & & & 1.755 & 41 & $14.1-25.1$ & 2003Var \\
\hline & $3 \mathrm{~b}$ & 21.57 & 19 & 11.07 & 63 & 1.652 & 82 & & & & & & & 1.652 & 82 & $12.3-25.1$ & 2003Var \\
\hline & $3 a$ & 21.29 & 11 & 6.43 & 3 & 1.094 & 190 & & & & & & & 1.094 & 27 & $11.0-25.0$ & 2002Ish \\
\hline & $3 \mathrm{~b}$ & 21.57 & 22 & 7.29 & 8 & 1.191 & 620 & & & & & & & 1.191 & 79 & $11.0-25.0$ & 2002Ish \\
\hline${ }^{\text {nat }} \mathrm{S}$ & 0 & 20.31 & 10 & 5.48 & 37 & 0.858 & 44 & & & & & & & 0.858 & 44 & $17.2-23.6$ & $1965 \mathrm{Wyc}$ \\
\hline${ }^{40} \mathrm{Ar}$ & $3 a$ & 19.86 & 15 & 9.12 & 31 & 1.372 & 36 & & & & & & & 1.372 & 36 & $10.5-25.0$ & 2002Ish \\
\hline & $3 b$ & 20.12 & 37 & 10.65 & 87 & 1.471 & 103 & & & & & & & 1.471 & 103 & $10.5-25.0$ & 2002Ish \\
\hline${ }^{\text {nat }} \mathrm{K}$ & 13 & 21.12 & 2 & 6.89 & 8 & 0.418 & 4 & & & & & & & 0.418 & 4 & $16.0-25.9$ & $1974 \mathrm{Ve} 1$ \\
\hline${ }^{40} \mathrm{Ca}$ & 0 & 20.58 & 11 & 6.23 & 46 & 1.709 & 92 & & & & & & & 1.709 & 92 & $17.2-23.7$ & 1966Dol \\
\hline & $3 a$ & 20.12 & 5 & 5.08 & 20 & 1.198 & 27 & & & & & & & 1.198 & 27 & $17.2-24.0$ & 2003Ero \\
\hline & $3 b$ & 20.06 & 10 & 5.10 & 33 & 1.197 & 54 & & & & & & & 1.197 & 54 & $17.2-24.0$ & 2003Ero \\
\hline${ }^{42} \mathrm{Ca}$ & $3 a$ & 20.11 & 10 & 8.07 & 40 & 1.455 & 50 & & & & & & & 1.455 & 50 & $15.2-23.0$ & 2003Ero \\
\hline & $3 b$ & 20.23 & 16 & 7.63 & 68 & 1.426 & 101 & & & & & & & 1.426 & 101 & $15.2-23.0$ & 2003Ero \\
\hline${ }^{44} \mathrm{Ca}$ & $3 a$ & 19.60 & 18 & 11.33 & 76 & 1.732 & 78 & & & & & & & 1.732 & 78 & $15.5-26.0$ & 2003Ero \\
\hline & $3 b$ & 19.95 & 33 & 10.39 & 97 & 1.603 & 123 & & & & & & & 1.603 & 123 & $12.5-26.0$ & 2003Ero \\
\hline${ }^{48} \mathrm{Ca}$ & 0 & 19.70 & 14 & 6.23 & 90 & 1.474 & 180 & & & & & & & 1.474 & 180 & $17.9-21.6$ & $1987 \mathrm{OKe}$ \\
\hline & $3 a$ & 19.75 & 13 & 7.11 & 55 & 1.353 & 66 & & & & & & & 1.353 & 66 & $15.5-23.0$ & 2003Ero \\
\hline & $3 \mathrm{~b}$ & 19.62 & 22 & 6.60 & 85 & 1.293 & 128 & & & & & & & 1.293 & 128 & $15.5-23.0$ & 2003Ero \\
\hline${ }^{\text {nat }} \mathrm{Ca}$ & 0 & 20.06 & 2 & 4.89 & 7 & 1.205 & 12 & & & & & & & 1.205 & 12 & $15.1-24.0$ & 1975Ahr \\
\hline & 0 & 19.93 & 3 & 4.89 & 8 & 1.163 & 14 & & & & & & & 1.163 & 14 & $15.2-24.0$ & 1972Ahr \\
\hline & 0 & 19.59 & 3 & 4.61 & 9 & 1.032 & 13 & & & & & & & 1.032 & 13 & $15.0-24.0$ & 1968Bez \\
\hline & $1 \mathrm{a}$ & 20.52 & 8 & 3.32 & 15 & 0.674 & 17 & & & & & & & 0.674 & 17 & $15.4-24.0$ & $1965 \mathrm{Wyc}$ \\
\hline & $1 \mathrm{~b}$ & 20.29 & 12 & 3.22 & 24 & 0.668 & 31 & & & & & & & 0.668 & 31 & $15.4-24.0$ & $1965 \mathrm{Wyc}$ \\
\hline & 2 & 19.98 & 4 & 4.82 & 2 & 1.128 & 110 & & & & & & & 1.128 & 19 & $9.4-24.0$ & 1985Ahr \\
\hline${ }^{46} \mathrm{Ti}$ & $3 a$ & 19.96 & 8 & 6.92 & 19 & 1.246 & 22 & & & & & & & 1.246 & 22 & $13.2-25.0$ & 2002Ish \\
\hline & $3 \mathrm{~b}$ & 19.79 & 20 & 7.54 & 52 & 1.282 & 73 & & & & & & & 1.282 & 73 & $13.2-25.0$ & 2002Ish \\
\hline${ }^{48} \mathrm{Ti}$ & $3 a$ & 19.78 & 17 & 8.42 & 53 & 1.179 & 56 & & & & & & & 1.179 & 56 & $14.5-23.0$ & 2002Ish \\
\hline & $3 \mathrm{~b}$ & 19.90 & 38 & 9.21 & 1.24 & 1.248 & 150 & & & & & & & 1.248 & 150 & $14.5-23.0$ & 2002Ish \\
\hline${ }^{51} \mathrm{~V}$ & 4 & 17.90 & 6 & 4.55 & 14 & 0.569 & 36 & 21.26 & 13 & 4.37 & 76 & 0.239 & 52 & 0.808 & 63 & $14.1-22.9$ & 1962Fu1 \\
\hline & $3 a$ & 17.71 & 13 & 3.46 & 1.14 & 0.279 & 179 & 22.03 & 59 & 11.41 & 185 & 1.739 & 392 & 2.018 & 431 & $15.2-25.0$ & 2003Var \\
\hline & $3 b$ & 17.76 & 23 & 4.08 & 1.52 & 0.447 & 349 & 22.37 & 60 & 8.83 & 473 & 1.292 & 827 & 1.739 & 898 & $15.2-25.0$ & 2003Var \\
\hline & $4 a$ & 17.36 & 4 & 2.84 & 6 & 0.332 & 7 & 19.93 & 2 & 1.10 & 5 & 0.143 & 6 & 0.475 & 9 & $11.0-24.5$ & 1969Gor \\
\hline & $4 \mathrm{~b}$ & 18.38 & 2 & 3.93 & 5 & 0.597 & 6 & 110.63 & 19790 & 47.90 & 7379 & 0.000 & 141 & 0.597 & 141 & $11.0-24.5$ & 1969Gor \\
\hline${ }^{52} \mathrm{Cr}$ & $3 a$ & 19.16 & 7 & 6.19 & 20 & 1.020 & 22 & & & & & & & 1.020 & 22 & $14.3-23.0$ & 2002Ish \\
\hline & $3 \mathrm{~b}$ & 19.24 & 18 & 6.88 & 51 & 1.068 & 66 & & & & & & & 1.068 & 66 & $14.3-23.0$ & 2002Ish \\
\hline & $4 \mathrm{a}$ & 18.24 & 4 & 5.04 & 9 & 0.791 & 11 & & & & & & & 0.791 & 11 & $14.1-23.0$ & 1969Gor \\
\hline & $4 \mathrm{~b}$ & 18.55 & 5 & 5.91 & 14 & 0.929 & 20 & & & & & & & 0.929 & 20 & $14.1-23.0$ & 1969Gor \\
\hline${ }^{55} \mathrm{Mn}$ & 4 & 16.43 & 6 & 2.95 & 32 & 0.153 & 31 & 19.77 & 17 & 8.61 & 43 & 0.860 & 59 & 1.013 & 67 & $14.0-23.0$ & 1979Al2 \\
\hline & 4 & 16.87 & 14 & 3.26 & 44 & 0.305 & 94 & 19.80 & 41 & 6.49 & 71 & 0.677 & 129 & 0.982 & 159 & $14.1-22.8$ & 1974Car \\
\hline & $4 a$ & 15.96 & 8 & 3.76 & 18 & 0.323 & 31 & 18.91 & 11 & 5.00 & 21 & 0.446 & 36 & 0.769 & 47 & $10.1-23.0$ & 1970Is1 \\
\hline & $4 \mathrm{~b}$ & 15.97 & 8 & 3.78 & 19 & 0.329 & 35 & 18.93 & 12 & 4.94 & 31 & 0.438 & 45 & 0.767 & 57 & $10.1-23.0$ & 1970Is1 \\
\hline${ }^{54} \mathrm{Fe}$ & 0 & 19.35 & 8 & 5.50 & 28 & 1.570 & 49 & & & & & & & 1.570 & 49 & $16.0-23.0$ & 1978 Nor \\
\hline${ }^{59} \mathrm{Co}$ & 4 & 16.43 & 7 & 2.73 & 37 & 0.138 & 39 & 18.64 & 20 & 7.31 & 31 & 0.747 & 52 & 0.885 & 65 & $14.0-20.9$ & 1979Al2 \\
\hline & 4 & 15.35 & 11 & 3.00 & 50 & 0.260 & 86 & 18.21 & 30 & 5.93 & 73 & 0.674 & 126 & 0.934 & 152 & $14.1-22.8$ & 1964Baz \\
\hline & $4 a$ & 16.21 & 4 & 2.79 & 18 & 0.169 & 21 & 18.83 & 8 & 6.48 & 11 & 0.724 & 26 & 0.893 & 34 & $12.1-24.0$ & 1969Gor \\
\hline & $4 \mathrm{~b}$ & 16.21 & 4 & 2.79 & 18 & 0.169 & 20 & 18.83 & 8 & 6.49 & 11 & 0.725 & 26 & 0.893 & 33 & $12.1-24.0$ & 1969Gor \\
\hline${ }^{58} \mathrm{Ni}$ & $3 a$ & 18.78 & 5 & 5.57 & 15 & 0.885 & 14 & & & & & & & 0.885 & 14 & $14.1-22.0$ & 2003Var \\
\hline & $3 b$ & 18.66 & 10 & 5.50 & 30 & 0.873 & 38 & & & & & & & 0.873 & 38 & $14.1-22.0$ & 2003Var \\
\hline & $3 a$ & 18.98 & 10 & 6.50 & 32 & 0.987 & 32 & & & & & & & 0.987 & 32 & $14.4-22.0$ & 2002Ish \\
\hline
\end{tabular}


Table 1 (continued)

\begin{tabular}{|c|c|c|c|c|c|c|c|c|c|c|c|c|c|c|c|c|c|}
\hline \multirow{2}{*}{$\begin{array}{l}\text { Nucl } \\
\end{array}$} & \multirow{2}{*}{$\frac{\mathrm{Id}}{3 \mathrm{~b}}$} & \multicolumn{2}{|c|}{$\begin{array}{c}E_{r, 1} \\
(\mathrm{MeV})\end{array}$} & \multicolumn{2}{|c|}{$\begin{array}{c}\Gamma_{r, 1} \\
(\mathrm{MeV})\end{array}$} & \multicolumn{2}{|l|}{$s_{1}$} & \multicolumn{2}{|c|}{$\begin{array}{c}E_{r, 2} \\
(\mathrm{MeV})\end{array}$} & $\begin{array}{c}\Gamma_{r} \\
(\mathrm{Me}\end{array}$ & & $s_{2}$ & & $s$ & & $\begin{array}{c}\varepsilon_{\min }-\varepsilon_{\max } \\
(\mathrm{MeV})\end{array}$ & Ref \\
\hline & & 18.74 & 20 & 6.18 & 63 & 0.945 & 78 & & & & & & & 0.945 & 78 & $14.4-22.0$ & 2002Ish \\
\hline & 4 & 18.26 & 6 & 6.95 & 17 & 0.294 & 6 & & & & & & & 0.294 & 6 & $12.2-21.8$ & 1974Fu3 \\
\hline & 4 & 18.79 & 2 & 7.92 & 6 & 0.401 & 3 & & & & & & & 0.401 & 3 & $14.1-22.0$ & 1970Gor \\
\hline${ }^{60} \mathrm{Ni}$ & $3 a$ & 16.69 & 18 & 3.47 & 41 & 0.382 & 104 & 19.57 & 28 & 5.26 & 62 & 0.656 & 137 & 1.038 & 172 & $12.1-21.0$ & 2003Var \\
\hline & $3 \mathrm{~b}$ & 16.46 & 19 & 2.69 & 96 & 0.211 & 143 & 19.05 & 43 & 5.93 & 80 & 0.870 & 204 & 1.080 & 249 & $12.1-21.0$ & 2003Var \\
\hline & 4 & 16.30 & 9 & 2.45 & 70 & 0.147 & 85 & 18.49 & 39 & 6.26 & 48 & 0.592 & 120 & 0.739 & 147 & $14.0-20.9$ & 1974Fu3 \\
\hline & 4 & 16.56 & 6 & 2.54 & 14 & 0.168 & 17 & 19.13 & 8 & 5.15 & 8 & 0.508 & 18 & 0.675 & 24 & $11.4-22.0$ & 1970Gor \\
\hline${ }^{63} \mathrm{Cu}$ & $3 a$ & 16.43 & 28 & 4.84 & 72 & 0.646 & 213 & 20.15 & 34 & 5.52 & 289 & 0.456 & 333 & 1.102 & 395 & $14.0-21.0$ & 2003Var \\
\hline & $3 \mathrm{~b}$ & 16.35 & 64 & 4.59 & 2.57 & 0.566 & 742 & 20.12 & 101 & 6.75 & 1042 & 0.602 & 1362 & 1.168 & 1551 & $14.0-21.0$ & 2003Var \\
\hline & $3 a$ & 16.28 & 50 & 3.70 & 238 & 0.400 & 592 & 20.23 & 90 & 7.23 & 958 & 0.790 & 1349 & 1.190 & 1474 & $14.5-21.0$ & 2002Ish \\
\hline & $3 \mathrm{~b}$ & 16.29 & 58 & 3.80 & 279 & 0.424 & 666 & 20.26 & 102 & 6.84 & 1084 & 0.737 & 1511 & 1.161 & 1651 & $14.5-21.0$ & 2002Ish \\
\hline & 4 & 16.25 & 10 & 4.64 & 31 & 0.469 & 53 & 19.62 & 19 & 4.47 & 120 & 0.191 & 69 & 0.660 & 87 & $14.2-20.7$ & 1964Fu1 \\
\hline & 5 & 16.19 & 34 & 2.39 & 224 & 0.107 & 171 & 17.93 & 171 & 11.36 & 505 & 0.891 & 253 & 0.997 & 306 & $14.2-20.7$ & 2016Va1 \\
\hline & 6 & 16.72 & 10 & 4.17 & 16 & 0.460 & 42 & 19.08 & 15 & 3.43 & 40 & 0.166 & 41 & 0.626 & 59 & $14.0-21.0$ & 1968Su1 \\
\hline${ }^{65} \mathrm{Cu}$ & $3 a$ & 16.92 & 7 & 8.09 & 38 & 1.139 & 37 & & & & & & & 1.139 & 37 & $14.2-21.0$ & 2003Var \\
\hline & $3 \mathrm{~b}$ & 16.85 & 26 & 7.46 & 119 & 1.079 & 154 & & & & & & & 1.079 & 154 & $14.2-21.0$ & 2003Var \\
\hline & $3 a$ & 17.14 & 22 & 10.27 & 150 & 1.537 & 176 & & & & & & & 1.537 & 176 & $14.5-21.0$ & 2002Ish \\
\hline & $3 \mathrm{~b}$ & 16.96 & 37 & 8.31 & 206 & 1.317 & 277 & & & & & & & 1.317 & 277 & $14.5-21.0$ & 2002Ish \\
\hline & 4 & 16.68 & 6 & 6.78 & 27 & 0.822 & 27 & & & & & & & 0.822 & 27 & $14.2-19.9$ & 1964Fu1 \\
\hline & 5 & 17.02 & 18 & 8.71 & 80 & 1.030 & 71 & & & & & & & 1.030 & 71 & $14.2-20.9$ & 2016Va1 \\
\hline${ }^{\text {nat }} \mathrm{Cu}$ & $1 \mathrm{a}$ & 18.12 & 10 & 5.61 & 19 & 0.919 & 22 & & & & & & & 0.919 & 22 & $14.4-24.9$ & $1965 \mathrm{Wyc}$ \\
\hline & $1 \mathrm{~b}$ & 17.90 & 25 & 6.14 & 68 & 0.965 & 91 & & & & & & & 0.965 & 91 & $14.4-24.9$ & $1965 \mathrm{Wyc}$ \\
\hline & 4 & 17.24 & 4 & 8.12 & 16 & 0.899 & 12 & & & & & & & 0.899 & 12 & $14.2-24.7$ & $1964 \mathrm{Ful}$ \\
\hline & $4 a$ & 17.17 & 8 & 9.97 & 31 & 1.460 & 35 & & & & & & & 1.460 & 35 & $14.1-25.0$ & 1970Is2 \\
\hline & $4 \mathrm{~b}$ & 17.03 & 7 & 8.24 & 39 & 1.248 & 48 & & & & & & & 1.248 & 48 & $14.1-25.0$ & 1970Is2 \\
\hline & $4 a$ & 15.16 & 103 & 12.81 & 151 & 1.613 & 223 & & & & & & & 1.613 & 223 & $14.6-23.2$ & 1968Tom \\
\hline & $4 \mathrm{~b}$ & 14.98 & 149 & 13.44 & 306 & 1.696 & 421 & & & & & & & 1.696 & 421 & $14.6-23.2$ & 1968Tom \\
\hline${ }^{64} \mathrm{Zn}$ & 8 & 16.23 & 13 & 3.25 & 48 & 0.220 & 74 & 19.16 & 25 & 5.91 & 89 & 0.533 & 119 & 0.753 & 140 & $14.0-20.8$ & 1976Ca1 \\
\hline${ }^{65} \mathrm{Zn}$ & $1 \mathrm{a}$ & 16.17 & 11 & 3.06 & 44 & 0.173 & 47 & 19.04 & 21 & 6.50 & 34 & 0.585 & 62 & 0.758 & 78 & $12.0-21.0$ & 2003Rod \\
\hline & $1 \mathrm{~b}$ & 16.21 & 22 & 3.32 & 97 & 0.208 & 127 & 19.16 & 45 & 6.12 & 126 & 0.529 & 188 & 0.737 & 227 & $12.0-21.0$ & 2003Rod \\
\hline${ }^{70} \mathrm{Ge}$ & 0 & 15.16 & 18 & 5.92 & 45 & 1.432 & 81 & & & & & & & 1.432 & 81 & $10.0-20.0$ & $1975 \mathrm{Mcc}$ \\
\hline & 8 & 16.76 & 8 & 7.55 & 34 & 1.006 & 35 & & & & & & & 1.006 & 35 & $13.1-20.8$ & 1976Ca1 \\
\hline${ }^{72} \mathrm{Ge}$ & 0 & 17.88 & 16 & 5.71 & 39 & 1.409 & 70 & & & & & & & 1.409 & 70 & $10.0-24.0$ & $1975 \mathrm{Mcc}$ \\
\hline & 8 & 16.63 & 6 & 7.48 & 25 & 1.173 & 30 & & & & & & & 1.173 & 30 & $13.1-20.8$ & 1976Ca1 \\
\hline${ }^{74} \mathrm{Ge}$ & 8 & 14.51 & 11 & 2.01 & 81 & 0.074 & 55 & 17.03 & 27 & 7.97 & 49 & 1.158 & 108 & 1.232 & 121 & $13.1-20.8$ & $1976 \mathrm{Ca} 1$ \\
\hline & 0 & 14.49 & 149 & 3.47 & 323 & 0.151 & 320 & 16.99 & 77 & 7.72 & 209 & 1.383 & 425 & 1.533 & 532 & $12.0-20.0$ & $1975 \mathrm{Mcc}$ \\
\hline${ }^{76} \mathrm{Ge}$ & 8 & 15.48 & 38 & 4.37 & 211 & 0.381 & 462 & 18.87 & 228 & 10.99 & 242 & 1.104 & 595 & 1.485 & 753 & $13.1-20.8$ & 1976Ca1 \\
\hline & 0 & 16.40 & 16 & 7.04 & 43 & 1.140 & 108 & 24.63 & 107 & 10.86 & 346 & 0.748 & 296 & 1.888 & 315 & $10.0-24.0$ & $1975 \mathrm{Mcc}$ \\
\hline${ }^{75} \mathrm{As}$ & 4 & 14.98 & 13 & 3.66 & 53 & 0.217 & 82 & 17.59 & 28 & 7.12 & 39 & 0.760 & 109 & 0.977 & 136 & $13.1-20.9$ & 1969Be1 \\
\hline & 8 & 15.19 & 29 & 4.43 & 102 & 0.419 & 273 & 18.12 & 80 & 7.66 & 126 & 0.819 & 353 & 1.238 & 446 & $13.1-20.8$ & $1976 \mathrm{Ca} 1$ \\
\hline${ }^{76} \mathrm{Se}$ & 0 & 15.67 & 8 & 6.33 & 32 & 1.337 & 50 & & & & & & & 1.337 & 50 & $13.1-19.7$ & 1978Gur \\
\hline & 8 & 16.69 & 8 & 9.38 & 40 & 1.398 & 48 & & & & & & & 1.398 & 48 & $13.1-20.8$ & $1976 \mathrm{Ca} 1$ \\
\hline${ }^{78} \mathrm{Se}$ & 8 & 14.97 & 16 & 3.91 & 61 & 0.376 & 113 & 18.42 & 28 & 6.19 & 100 & 0.671 & 161 & 1.047 & 197 & $13.1-20.8$ & 1976Ca1 \\
\hline${ }^{80} \mathrm{Se}$ & 5 & 16.60 & 6 & 6.80 & 25 & 1.255 & 35 & & & & & & & 1.255 & 35 & $13.1-20.0$ & 2016Va1 \\
\hline & 8 & 16.16 & 11 & 5.51 & 36 & 1.004 & 63 & & & & & & & 1.004 & 63 & $13.1-17.0$ & 1976Ca1 \\
\hline${ }^{82} \mathrm{Se}$ & 0 & 16.00 & 5 & 5.68 & 21 & 1.308 & 35 & & & & & & & 1.308 & 35 & $13.1-19.9$ & 1978Gur \\
\hline & 8 & 16.63 & 5 & 5.80 & 17 & 1.125 & 24 & & & & & & & 1.125 & 24 & $13.1-20.8$ & 1976Ca1 \\
\hline${ }^{\text {nat }} \mathrm{Rb}$ & 4 & 16.73 & 2 & 4.25 & 4 & 1.012 & 9 & & & & & & & 1.012 & 9 & $10.6-17.9$ & 1971Lep \\
\hline${ }^{\text {nat }} \mathrm{Sr}$ & 4 & 16.79 & 1 & 4.32 & 4 & 1.079 & 8 & & & & & & & 1.079 & 8 & $10.9-17.9$ & 1971Lep \\
\hline${ }^{89} \mathrm{Y}$ & 4 & 16.74 & 1 & 4.23 & 3 & 1.135 & 7 & & & & & & & 1.135 & 7 & $14.0-19.0$ & 1971Le1 \\
\hline & $3 a$ & 16.80 & 6 & 4.49 & 28 & 1.253 & 55 & & & & & & & 1.253 & 55 & $15.3-19.0$ & 2003Var \\
\hline & $3 \mathrm{~b}$ & 16.80 & 22 & 4.50 & 101 & 1.255 & 255 & & & & & & & 1.255 & 255 & $15.3-19.0$ & 2003Var \\
\hline & 4 & 17.52 & 1 & 4.61 & 4 & 0.376 & 3 & & & & & & & 0.376 & 3 & $15.3-19.0$ & 1999Bel \\
\hline & 4 & 16.78 & 1 & 3.92 & 2 & 0.861 & 3 & & & & & & & 0.861 & 3 & $14.0-18.9$ & 1967Be2 \\
\hline & 12 & 16.83 & 3 & 3.68 & 8 & 0.893 & 16 & & & & & & & 0.893 & 16 & $14.0-18.1$ & 1972You \\
\hline${ }^{90} \mathrm{Zr}$ & $3 a$ & 16.82 & 5 & 3.99 & 23 & 1.192 & 47 & & & & & & & 1.192 & 47 & $14.9-18.5$ & 2003Var \\
\hline & $3 \mathrm{~b}$ & 16.79 & 15 & 3.85 & 62 & 1.164 & 164 & & & & & & & 1.164 & 164 & $14.9-18.5$ & 2003Var \\
\hline & 4 & 16.84 & 2 & 4.10 & 8 & 0.958 & 15 & & & & & & & 0.958 & 15 & $15.0-18.5$ & 1972Ask \\
\hline & 4 & 16.84 & 1 & 3.99 & 3 & 0.861 & 5 & & & & & & & 0.861 & 5 & $14.0-18.9$ & 1967Be2 \\
\hline & 8 & 16.73 & 1 & 4.14 & 3 & 1.025 & 6 & & & & & & & 1.025 & 6 & $14.0-19.0$ & 1971Le1 \\
\hline${ }^{91} \mathrm{Zr}$ & 4 & 16.58 & 2 & 4.17 & 7 & 0.892 & 10 & & & & & & & 0.892 & 10 & $14.0-18.9$ & 1967Be2 \\
\hline & 5 & 16.58 & 8 & 4.12 & 30 & 0.884 & 42 & & & & & & & 0.884 & 42 & $14.0-18.9$ & 2015Var \\
\hline${ }^{92} \mathrm{Zr}$ & 4 & 16.26 & 2 & 4.64 & 9 & 0.885 & 12 & & & & & & & 0.885 & 12 & $14.0-18.9$ & 1967Be2 \\
\hline${ }^{94} \mathrm{Zr}$ & 4 & 16.21 & 2 & 5.25 & 11 & 0.956 & 15 & & & & & & & 0.956 & 15 & $14.0-18.9$ & $1967 \mathrm{Be} 2$ \\
\hline & 5 & 16.35 & 10 & 5.18 & 48 & 0.993 & 64 & & & & & & & 0.993 & 64 & $14.0-19.0$ & 2015Var \\
\hline${ }^{93} \mathrm{Nb}$ & 8 & 16.58 & 1 & 4.95 & 6 & 1.132 & 10 & & & & & & & 1.132 & 10 & $14.0-19.0$ & 1971Le1 \\
\hline${ }^{92} \mathrm{Mo}$ & $3 a$ & 17.16 & 6 & 4.68 & 22 & 1.287 & 42 & & & & & & & 1.287 & 42 & $14.4-19.0$ & 2003Var \\
\hline
\end{tabular}


Table 1 (continued)

\begin{tabular}{|c|c|c|c|c|c|c|c|c|c|c|c|c|c|c|c|}
\hline \multirow[t]{5}{*}{ Nucl } & Id & \multicolumn{2}{|c|}{$\begin{array}{c}E_{r, 1} \\
(\mathrm{MeV})\end{array}$} & \multicolumn{2}{|c|}{$\begin{array}{c}\Gamma_{r, 1} \\
(\mathrm{MeV}) \\
\end{array}$} & \multicolumn{2}{|l|}{$s_{1}$} & $\begin{array}{c}E_{r, 2} \\
(\mathrm{MeV}) \\
\end{array}$ & $\begin{array}{c}\Gamma_{r, 2} \\
(\mathrm{MeV}) \\
\end{array}$ & \multicolumn{2}{|l|}{$s_{2}$} & $s$ & & $\begin{array}{c}\varepsilon_{\min }-\varepsilon_{\max } \\
(\mathrm{MeV})\end{array}$ & Ref \\
\hline & $3 \mathrm{~b}$ & 17.10 & 14 & 4.34 & 55 & 1.227 & 135 & & & & & 1.227 & 135 & $14.4-19.0$ & 2003Var \\
\hline & 4 & 16.82 & 1 & 4.11 & 4 & 0.756 & 5 & & & & & 0.756 & 5 & $14.0-18.9$ & 1974Be3 \\
\hline & $4 a$ & 16.55 & 4 & 5.03 & 21 & 0.944 & 27 & & & & & 0.944 & 27 & $14.5-19.0$ & 1970Is3 \\
\hline & $4 \mathrm{~b}$ & 16.51 & 4 & 4.99 & 26 & 0.950 & 36 & & & & & 0.950 & 36 & $14.5-19.0$ & $1970 \mathrm{Is} 3$ \\
\hline${ }^{94} \mathrm{Mo}$ & 4 & 16.53 & 2 & 5.12 & 5 & 1.113 & 8 & & & & & 1.113 & 8 & $9.6-18.9$ & 1974Be3 \\
\hline${ }^{96} \mathrm{Mo}$ & 4 & 16.11 & 4 & 5.64 & 15 & 1.155 & 29 & & & & & 1.155 & 29 & $13.2-17.0$ & 1974Be3 \\
\hline${ }^{98} \mathrm{Mo}$ & 4 & 15.79 & 3 & 5.90 & 16 & 1.211 & 24 & & & & & 1.211 & 24 & $13.2-18.9$ & 1974Be3 \\
\hline & $4 a$ & 15.54 & 5 & 5.32 & 21 & 1.300 & 33 & & & & & 1.300 & 33 & $13.1-19.0$ & 1970Is3 \\
\hline & $4 \mathrm{~b}$ & 15.53 & 9 & 5.44 & 37 & 1.346 & 72 & & & & & 1.346 & 72 & $13.1-19.0$ & 1970Is3 \\
\hline${ }^{100} \mathrm{Mo}$ & 9 & 15.72 & 3 & 7.68 & 14 & 1.404 & 19 & & & & & 1.404 & 19 & $12.1-20.0$ & $1974 \mathrm{Be} 3$ \\
\hline${ }^{103} \mathrm{Rh}$ & $3 a$ & 16.24 & 7 & 7.49 & 39 & 1.486 & 57 & & & & & 1.486 & 57 & $13.1-19.0$ & 2003Var \\
\hline & $3 \mathrm{~b}$ & 16.23 & 17 & 7.60 & 81 & 1.500 & 138 & & & & & 1.500 & 138 & $13.1-19.0$ & 2003Var \\
\hline & 4 & 16.14 & 3 & 7.22 & 17 & 1.414 & 26 & & & & & 1.414 & 26 & $13.2-18.9$ & 1974Le1 \\
\hline & 4 & 16.55 & 18 & 5.92 & 9 & 1.576 & 360 & & & & & 1.576 & 86 & $10.0-19.0$ & 1962Bog \\
\hline${ }^{108} \mathrm{Pd}$ & 0 & 14.97 & 43 & 5.42 & 46 & 0.771 & 263 & $18.13 \quad 36$ & $4.03 \quad 285$ & 0.413 & 382 & 1.184 & 464 & $10.0-19.0$ & 1969Dea \\
\hline${ }^{\text {nat }} \mathrm{Pd}$ & 4 & 15.89 & 3 & 6.30 & 9 & 1.279 & 16 & & & & & 1.279 & 16 & $10.2-17.8$ & 1971Lep \\
\hline${ }^{107} \mathrm{Ag}$ & 4 & 15.83 & 4 & 6.49 & 12 & 1.193 & 17 & & & & & 1.193 & 17 & $9.5-19.0$ & 1969Ish \\
\hline & 4 & 15.89 & 4 & 6.65 & 18 & 0.986 & 23 & & & & & 0.986 & 23 & $13.1-18.7$ & 1969Be1 \\
\hline & $4 a$ & 15.68 & 6 & 5.56 & 3 & 1.384 & 180 & & & & & 1.384 & 32 & $10.0-19.0$ & 1962Bog \\
\hline & $4 \mathrm{~b}$ & 15.60 & 9 & 5.43 & 5 & 1.343 & 220 & & & & & 1.343 & 48 & $10.0-19.0$ & 1962Bog \\
\hline${ }^{109} \mathrm{Ag}$ & 4 & 13.54 & 19 & 3.49 & 167 & 0.275 & 165 & $16.62 \quad 18$ & $4.41 \quad 46$ & 0.490 & 124 & 0.765 & 206 & $13.1-19.0$ & 1969Ish \\
\hline${ }^{\text {nat }} \mathrm{Ag}$ & 4 & 16.06 & 2 & 7.33 & 8 & 1.441 & 12 & & & & & 1.441 & 12 & $13.2-18.9$ & 1971Lep \\
\hline${ }^{n a t} \mathrm{Cd}$ & 4 & 15.77 & 1 & 5.70 & 4 & 1.227 & 7 & & & & & 1.227 & 7 & $10.2-17.8$ & 1971Lep \\
\hline${ }^{115} \mathrm{In}$ & 4 & 15.72 & 1 & 5.57 & 6 & 1.273 & 10 & & & & & 1.273 & 10 & $13.2-17.8$ & 1974Le1 \\
\hline & 4 & 15.63 & 1 & 5.22 & 5 & 1.285 & 10 & & & & & 1.285 & 10 & $13.1-17.8$ & 1969Fu1 \\
\hline & 4 & 15.39 & 14 & 4.67 & 7 & 1.374 & 270 & & & & & 1.374 & 69 & $10.0-18.0$ & 1962Bog \\
\hline & 5 & 15.53 & 6 & 4.79 & 28 & 1.180 & 47 & & & & & 1.180 & 47 & $13.1-17.8$ & 2013Var \\
\hline${ }^{112} \mathrm{Sn}$ & 4 & 15.62 & 5 & 5.01 & 3 & 1.246 & 130 & & & & & 1.246 & 25 & $10.9-18.0$ & 1974Sor \\
\hline & 5 & 15.63 & 5 & 5.30 & 23 & 1.300 & 43 & & & & & 1.300 & 43 & $13.1-18.0$ & 2009Var \\
\hline${ }^{114} \mathrm{Sn}$ & 4 & 15.82 & 4 & 6.08 & 19 & 1.378 & 36 & & & & & 1.378 & 36 & $13.1-18.0$ & 1975Sor \\
\hline & 5 & 15.77 & 4 & 5.91 & 18 & 1.337 & 34 & & & & & 1.337 & 34 & $13.1-18.0$ & 2009Var \\
\hline${ }^{116} \mathrm{Sn}$ & 4 & 15.55 & 1 & 5.06 & 6 & 1.254 & 11 & & & & & 1.254 & 11 & $13.1-17.9$ & 1974Le1 \\
\hline & 4 & 15.67 & 2 & 4.17 & 7 & 1.017 & 11 & & & & & 1.017 & 11 & $13.0-18.0$ & 1969Fu1 \\
\hline & $4 a$ & 15.73 & 3 & 5.59 & 13 & 1.299 & 25 & & & & & 1.299 & 25 & $13.1-18.0$ & 1975Sor \\
\hline & $4 \mathrm{~b}$ & 15.73 & 3 & 5.61 & 14 & 1.304 & 26 & & & & & 1.304 & 26 & $13.1-18.0$ & 1975Sor \\
\hline & 5 & 15.58 & 1 & 4.73 & 3 & 1.162 & 6 & & & & & 1.162 & 6 & $13.1-18.0$ & 2009Var \\
\hline${ }^{117} \mathrm{Sn}$ & 4 & 15.64 & 2 & 5.02 & 9 & 1.181 & 17 & & & & & 1.181 & 17 & $13.2-17.8$ & 1974Le1 \\
\hline & 4 & 15.65 & 1 & 5.00 & 6 & 1.153 & 9 & & & & & 1.153 & 9 & $13.1-17.9$ & 1969Fu1 \\
\hline & $4 a$ & 15.60 & 3 & 5.16 & 13 & 1.216 & 25 & & & & & 1.216 & 25 & $13.1-18.0$ & 1975Sor \\
\hline & $4 \mathrm{~b}$ & 15.63 & 4 & 5.27 & 16 & 1.242 & 33 & & & & & 1.242 & 33 & $13.1-18.0$ & 1975Sor \\
\hline & 5 & 15.64 & 1 & 4.98 & 3 & 1.166 & 5 & & & & & 1.166 & 5 & $13.1-18.0$ & 2009Var \\
\hline${ }^{118} \mathrm{Sn}$ & 4 & 15.43 & 1 & 4.84 & 6 & 1.221 & 11 & & & & & 1.221 & 11 & $13.1-17.9$ & 1974Le1 \\
\hline & 4 & 15.59 & 1 & 4.75 & 4 & 1.097 & 7 & & & & & 1.097 & 7 & $13.1-17.9$ & 1969Fu1 \\
\hline & $4 a$ & 15.37 & 4 & 6.02 & 19 & 1.478 & 40 & & & & & 1.478 & 40 & $13.1-18.0$ & 1974Sor \\
\hline & $4 \mathrm{~b}$ & 15.38 & 4 & 6.08 & 23 & 1.491 & 50 & & & & & 1.491 & 50 & $13.1-18.0$ & 1974Sor \\
\hline & 5 & 15.48 & 1 & 4.56 & 2 & 1.086 & 3 & & & & & 1.086 & 3 & $13.1-18.0$ & 2009Var \\
\hline${ }^{119} \mathrm{Sn}$ & 4 & 15.53 & 2 & 4.78 & 6 & 1.085 & 11 & & & & & 1.085 & 11 & $13.0-17.9$ & 1969Fu1 \\
\hline & $4 a$ & 14.74 & 3 & 4.75 & 13 & 0.971 & 21 & & & & & 0.971 & 21 & $13.1-17.9$ & 1975Sor \\
\hline & $4 \mathrm{~b}$ & 14.72 & 4 & 4.60 & 16 & 0.945 & 29 & & & & & 0.945 & 29 & $13.1-17.9$ & 1975Sor \\
\hline & 5 & 15.58 & 1 & 5.23 & 5 & 1.190 & 8 & & & & & 1.190 & 8 & $13.1-18.0$ & 2009Var \\
\hline${ }^{120} \mathrm{Sn}$ & 4 & 15.37 & 1 & 5.08 & 6 & 1.295 & 12 & & & & & 1.295 & 12 & $13.1-17.9$ & 1974Le1 \\
\hline & 4 & 15.40 & 1 & 4.86 & 4 & 1.219 & 8 & & & & & 1.219 & 8 & $13.1-17.9$ & 1969Fu1 \\
\hline & $4 a$ & 15.30 & 4 & 5.84 & 21 & 1.465 & 43 & & & & & 1.465 & 43 & $13.1-18.0$ & 1974Sor \\
\hline & $4 \mathrm{~b}$ & 15.27 & 6 & 5.72 & 29 & 1.435 & 66 & & & & & 1.435 & 66 & $13.1-18.0$ & 1974Sor \\
\hline & 5 & 15.34 & 1 & 4.79 & 2 & 1.206 & 4 & & & & & 1.206 & 4 & $13.1-18.0$ & 2009Var \\
\hline${ }^{122} \mathrm{Sn}$ & $4 a$ & 15.34 & 3 & 4.73 & 11 & 1.120 & 22 & & & & & 1.120 & 22 & $13.1-18.0$ & 1975Sor \\
\hline & $4 \mathrm{~b}$ & 15.35 & 5 & 4.74 & 15 & 1.125 & 36 & & & & & 1.125 & 36 & $13.1-18.0$ & 1975Sor \\
\hline & 5 & 15.25 & 2 & 4.72 & 7 & 1.082 & 10 & & & & & 1.082 & 10 & $13.1-18.0$ & 2009Var \\
\hline${ }^{124} \mathrm{Sn}$ & $3 a$ & 15.31 & 5 & 4.94 & 22 & 1.173 & 34 & & & & & 1.173 & 34 & $13.1-18.0$ & 2003Var \\
\hline & $3 b$ & 15.29 & 8 & 4.86 & 36 & 1.162 & 66 & & & & & 1.162 & 66 & $13.1-18.0$ & 2003Var \\
\hline & 4 & 15.27 & 2 & 4.77 & 8 & 1.150 & 14 & & & & & 1.150 & 14 & $13.2-17.8$ & 1974Le1 \\
\hline & 4 & 15.18 & 2 & 4.79 & 7 & 1.183 & 14 & & & & & 1.183 & 14 & $13.1-17.8$ & 1969Fu1 \\
\hline & $4 a$ & 15.19 & 4 & 5.45 & 18 & 1.254 & 30 & & & & & 1.254 & 30 & $13.1-18.0$ & 1975Sor \\
\hline & $4 \mathrm{~b}$ & 15.19 & 6 & 5.43 & 23 & 1.253 & 49 & & & & & 1.253 & 49 & $13.1-18.0$ & 1975Sor \\
\hline & 5 & 15.17 & 1 & 4.87 & 3 & 1.163 & 4 & & & & & 1.163 & 4 & $13.1-18.0$ & 2009Var \\
\hline${ }^{\text {nat }} \mathrm{Sb}$ & 4 & 15.48 & 1 & 5.01 & 3 & 1.242 & 6 & & & & & 1.242 & 6 & $10.2-17.8$ & 1971Lep \\
\hline${ }^{124} \mathrm{Te}$ & 6 & 15.23 & 2 & 5.50 & 8 & 1.337 & 15 & & & & & 1.337 & 15 & $12.0-18.9$ & 1976Le2 \\
\hline
\end{tabular}


Table 1 (continued)

\begin{tabular}{|c|c|c|c|c|c|c|c|c|c|c|c|c|c|c|c|c|c|}
\hline \multirow{2}{*}{$\begin{array}{c}\mathrm{Nucl} \\
{ }^{126} \mathrm{Te}\end{array}$} & \multirow{2}{*}{$\frac{\text { Id }}{6}$} & \multicolumn{2}{|c|}{$\begin{array}{c}E_{r, 1} \\
(\mathrm{MeV})\end{array}$} & \multicolumn{2}{|c|}{$\begin{array}{c}\Gamma_{r, 1} \\
(\mathrm{MeV})\end{array}$} & \multicolumn{2}{|l|}{$s_{1}$} & \multicolumn{2}{|c|}{$\begin{array}{c}E_{r, 2} \\
(\mathrm{MeV})\end{array}$} & $\begin{array}{r}\Gamma_{r}, \\
(\mathrm{Me}\end{array}$ & & $s_{2}$ & & $s$ & & $\begin{array}{c}\varepsilon_{\min }-\varepsilon_{\max } \\
(\mathrm{MeV})\end{array}$ & Ref \\
\hline & & 15.15 & 2 & 5.36 & 7 & 1.358 & 13 & & & & & & & 1.358 & 13 & $12.0-18.9$ & 1976Le2 \\
\hline${ }^{128} \mathrm{Te}$ & 6 & 15.12 & 2 & 5.30 & 8 & 1.367 & 14 & & & & & & & 1.367 & 14 & $12.0-18.9$ & 1976Le2 \\
\hline${ }^{130} \mathrm{Te}$ & 6 & 15.11 & 2 & 4.98 & 7 & 1.334 & 13 & & & & & & & 1.334 & 13 & $12.0-18.9$ & 1976Le2 \\
\hline${ }^{127} \mathrm{I}$ & 4 & 14.77 & 3 & 4.09 & 5 & 0.795 & 23 & 17.30 & 10 & 3.69 & 30 & 0.173 & 24 & 0.968 & 33 & $12.0-20.0$ & 1999Bel \\
\hline & 4 & 13.91 & 9 & 1.10 & 63 & 0.052 & 38 & 15.20 & 10 & 4.73 & 10 & 1.088 & 49 & 1.140 & 62 & $12.2-20.0$ & 1989Ras \\
\hline & 4 & 14.45 & 20 & 3.72 & 37 & 0.713 & 215 & 16.58 & 46 & 4.72 & 61 & 0.565 & 234 & 1.278 & 318 & $8.8-19.8$ & 1969Be6 \\
\hline & 4 & 14.25 & 15 & 3.28 & 39 & 0.340 & 122 & 16.30 & 30 & 5.15 & 34 & 0.632 & 142 & 0.972 & 187 & $12.1-19.9$ & $1966 \mathrm{Br} 1$ \\
\hline & 5 & 14.20 & 28 & 3.17 & 79 & 0.367 & 259 & 16.21 & 53 & 5.22 & 48 & 0.810 & 298 & 1.177 & 395 & $12.1-19.9$ & 2006Var \\
\hline & 8 & 14.61 & 20 & 2.62 & 104 & 0.163 & 196 & 15.72 & 50 & 6.19 & 115 & 1.012 & 142 & 1.175 & 243 & $12.1-16.9$ & 1987Ber \\
\hline${ }^{133} \mathrm{Cs}$ & 4 & 15.33 & 1 & 5.28 & 2 & 1.351 & 4 & & & & & & & 1.351 & 4 & $12.0-19.0$ & 1974Le1 \\
\hline & 4 & 15.24 & 2 & 4.97 & 8 & 1.155 & 12 & & & & & & & 1.155 & 12 & $12.1-18.7$ & 1969Be1 \\
\hline & 5 & 15.16 & 13 & 4.83 & 49 & 1.241 & 74 & & & & & & & 1.241 & 74 & $12.1-19.0$ & 2016Va2 \\
\hline${ }^{138} \mathrm{Ba}$ & 5 & 15.13 & 9 & 4.51 & 32 & 1.126 & 46 & & & & & & & 1.126 & 46 & $12.1-18.7$ & 2016Va2 \\
\hline & 4 & 15.25 & 1 & 4.58 & 5 & 1.176 & 9 & & & & & & & 1.176 & 9 & $12.1-18.7$ & 1970Be8 \\
\hline${ }^{\text {nat }} \mathrm{Ba}$ & 4 & 15.29 & 1 & 4.93 & 3 & 1.370 & 6 & & & & & & & 1.370 & 6 & $10.1-17.8$ & 1971Be4 \\
\hline${ }^{139} \mathrm{La}$ & 4 & 15.24 & 2 & 4.82 & 9 & 1.379 & 15 & & & & & & & 1.379 & 15 & $12.0-18.9$ & 1972De1 \\
\hline & 4 & 15.23 & 1 & 4.45 & 4 & 1.159 & 8 & & & & & & & 1.159 & 8 & $12.0-18.9$ & 1971Be4 \\
\hline & 4 & 15.13 & 2 & 4.45 & 8 & 1.253 & 17 & & & & & & & 1.253 & 17 & $12.1-19.0$ & 1968Be5 \\
\hline & $4 a$ & 14.43 & 7 & 3.60 & 20 & 0.783 & 23 & & & & & & & 0.783 & 23 & $12.5-19.0$ & 1964Ric \\
\hline & $4 \mathrm{~b}$ & 14.45 & 12 & 3.38 & 35 & 0.767 & 52 & & & & & & & 0.767 & 52 & $12.5-19.0$ & 1964Ric \\
\hline${ }^{140} \mathrm{Ce}$ & 6 & 15.03 & 1 & 4.39 & 4 & 1.292 & 9 & & & & & & & 1.292 & 9 & $12.0-18.9$ & 1976Le2 \\
\hline${ }^{142} \mathrm{Ce}$ & 6 & 14.85 & 2 & 5.08 & 8 & 1.284 & 15 & & & & & & & 1.284 & 15 & $12.0-18.9$ & 1976Le2 \\
\hline${ }^{141} \mathrm{Pr}$ & 4 & 15.19 & 1 & 4.23 & 8 & 1.106 & 18 & & & & & & & 1.106 & 18 & $12.1-16.9$ & 1987Ber \\
\hline & 4 & 15.14 & 2 & 4.40 & 6 & 1.083 & 10 & & & & & & & 1.083 & 10 & $12.1-18.7$ & 1966Br1 \\
\hline & $4 a$ & 14.66 & 7 & 3.82 & 21 & 0.844 & 25 & & & & & & & 0.844 & 25 & $12.5-19.0$ & 1964Ric \\
\hline & $4 \mathrm{~b}$ & 14.70 & 14 & 3.75 & 40 & 0.840 & 62 & & & & & & & 0.840 & 62 & $12.5-19.0$ & 1964Ric \\
\hline & 12 & 15.35 & 2 & 4.05 & 4 & 1.021 & 8 & & & & & & & 1.021 & 8 & $12.0-18.1$ & 1972You \\
\hline & 12 & 15.04 & 1 & 4.47 & 3 & 1.179 & 5 & & & & & & & 1.179 & 5 & $12.0-16.9$ & 1971Be4 \\
\hline & 12 & 15.23 & 1 & 3.98 & 4 & 1.031 & 7 & & & & & & & 1.031 & 7 & $12.1-19.0$ & 1970Su1 \\
\hline & 13 & 15.39 & 2 & 3.80 & 6 & 1.039 & 12 & & & & & & & 1.039 & 12 & $12.0-18.9$ & 1972De1 \\
\hline${ }^{142} \mathrm{Nd}$ & $3 a$ & 14.95 & 5 & 4.46 & 17 & 1.215 & 30 & & & & & & & 1.215 & 30 & $13.1-18.0$ & 2003Var \\
\hline & $3 \mathrm{~b}$ & 14.94 & 12 & 4.48 & 47 & 1.218 & 106 & & & & & & & 1.218 & 106 & $13.1-18.0$ & 2003Var \\
\hline & 4 & 14.94 & 1 & 4.41 & 3 & 1.195 & 7 & & & & & & & 1.195 & 7 & $12.0-18.9$ & $1971 \mathrm{Ca} 1$ \\
\hline${ }^{143} \mathrm{Nd}$ & 4 & 15.00 & 2 & 4.73 & 8 & 1.236 & 14 & & & & & & & 1.236 & 14 & $12.0-19.0$ & $1971 \mathrm{Ca} 1$ \\
\hline${ }^{144} \mathrm{Nd}$ & 4 & 15.04 & 2 & 5.25 & 7 & 1.239 & 12 & & & & & & & 1.239 & 12 & $12.0-18.9$ & $1971 \mathrm{Ca} 1$ \\
\hline${ }^{145} \mathrm{Nd}$ & 4 & 14.94 & 4 & 6.27 & 18 & 1.378 & 28 & & & & & & & 1.378 & 28 & $12.0-18.9$ & 1971Ca1 \\
\hline${ }^{146} \mathrm{Nd}$ & 4 & 14.73 & 2 & 5.74 & 10 & 1.314 & 17 & & & & & & & 1.314 & 17 & $12.0-18.9$ & $1971 \mathrm{Ca} 1$ \\
\hline & $4 \mathrm{a}$ & 13.94 & 3 & 3.89 & 9 & 1.002 & 14 & & & & & & & 1.002 & 14 & $12.2-19.0$ & 1971Vas \\
\hline & $4 \mathrm{~b}$ & 13.92 & 4 & 3.86 & 13 & 0.994 & 28 & & & & & & & 0.994 & 28 & $12.2-19.0$ & 1971Vas \\
\hline${ }^{148} \mathrm{Nd}$ & 4 & 12.78 & 26 & 4.03 & 69 & 0.326 & 126 & 15.49 & 19 & 5.22 & 39 & 0.827 & 143 & 1.153 & 191 & $10.8-18.6$ & $1971 \mathrm{Ca} 1$ \\
\hline & 4 & 13.67 & 3 & 2.68 & 19 & 0.855 & 53 & 15.96 & 4 & 1.22 & 18 & 0.137 & 26 & 0.992 & 59 & $13.0-17.0$ & 1969Vas \\
\hline${ }^{150} \mathrm{Nd}$ & 4 & 12.30 & 6 & 3.38 & 26 & 0.432 & 46 & 16.03 & 9 & 5.12 & 31 & 0.821 & 62 & 1.253 & 77 & $10.8-18.6$ & 1971Ca1 \\
\hline${ }^{144} \mathrm{Sm}$ & 4 & 15.31 & 2 & 4.42 & 7 & 1.251 & 13 & & & & & & & 1.251 & 13 & $12.1-18.9$ & $1974 \mathrm{Ca} 5$ \\
\hline${ }^{148} \mathrm{Sm}$ & 4 & 14.82 & 1 & 5.06 & 5 & 1.242 & 9 & & & & & & & 1.242 & 9 & $12.1-18.9$ & 1974Ca5 \\
\hline & $4 a$ & 14.01 & 2 & 3.80 & 1 & 0.919 & 50 & & & & & & & 0.919 & 11 & $7.7-19.0$ & 1971Vas \\
\hline & $4 \mathrm{~b}$ & 13.99 & 3 & 3.76 & 1 & 0.907 & 60 & & & & & & & 0.907 & 15 & $7.7-19.0$ & 1971Vas \\
\hline${ }^{150} \mathrm{Sm}$ & 4 & 14.59 & 3 & 5.92 & 11 & 1.324 & 18 & & & & & & & 1.324 & 18 & $12.1-18.9$ & $1974 \mathrm{Ca} 5$ \\
\hline & 4 & 13.92 & 3 & 4.49 & 10 & 1.160 & 19 & & & & & & & 1.160 & 19 & $12.2-19.0$ & 1969Vas \\
\hline${ }^{152} \mathrm{Sm}$ & 4 & 12.39 & 3 & 2.99 & 12 & 0.377 & 21 & 15.73 & 4 & 5.15 & 13 & 0.853 & 28 & 1.230 & 35 & $10.9-18.8$ & 1974Ca5 \\
\hline & 4 & 11.69 & 2 & 2.15 & 7 & 0.703 & 22 & 14.99 & 3 & 2.50 & 10 & 0.747 & 25 & 1.449 & 33 & $11.0-18.8$ & 1969Vas \\
\hline${ }^{154} \mathrm{Sm}$ & 0 & 12.17 & 20 & 2.80 & 95 & 0.360 & 187 & 15.63 & 49 & 5.89 & 151 & 0.873 & 286 & 1.233 & 342 & $10.9-18.6$ & $1981 \mathrm{Gur}$ \\
\hline & 4 & 12.27 & 3 & 2.97 & 15 & 0.381 & 26 & 15.94 & 6 & 5.62 & 20 & 0.845 & 37 & 1.226 & 45 & $11.0-18.6$ & $1974 \mathrm{Ca} 5$ \\
\hline & 4 & 11.28 & 4 & 1.92 & 14 & 0.270 & 20 & 14.90 & 3 & 4.06 & 9 & 0.995 & 21 & 1.265 & 29 & $11.0-18.8$ & 1969Vas \\
\hline${ }^{151} \mathrm{Eu}$ & $4 a$ & 13.88 & 5 & 4.69 & 14 & 0.850 & 23 & 14.45 & 7 & 0.75 & 34 & 0.035 & 19 & 0.885 & 30 & $10.2-18.0$ & 1971Vas \\
\hline & $4 \mathrm{~b}$ & 13.93 & 6 & 4.71 & 13 & 0.873 & 28 & 14.44 & 6 & 0.62 & 32 & 0.026 & 15 & 0.899 & 32 & $10.2-18.0$ & 1971Vas \\
\hline${ }^{153} \mathrm{Eu}$ & 4 & 12.33 & 4 & 2.77 & 17 & 0.305 & 27 & 15.78 & 7 & 5.76 & 19 & 0.892 & 40 & 1.197 & 48 & $10.9-18.7$ & 1969Be8 \\
\hline & $4 \mathrm{a}$ & 11.94 & 2 & 2.02 & 10 & 0.246 & 12 & 15.23 & 4 & 3.31 & 13 & 0.588 & 17 & 0.834 & 21 & $11.0-18.8$ & 1971Vas \\
\hline & $4 \mathrm{~b}$ & 11.96 & 3 & 2.09 & 10 & 0.262 & 14 & 15.16 & 6 & 2.96 & 17 & 0.531 & 29 & 0.792 & 32 & $11.0-18.8$ & 1971Vas \\
\hline${ }^{152} \mathrm{Gd}$ & $4 \mathrm{a}$ & 11.79 & 4 & 3.03 & 15 & 0.312 & 21 & 14.72 & 4 & 3.16 & 13 & 0.566 & 24 & 0.878 & 32 & $10.2-18.0$ & 1971Vas \\
\hline & $4 \mathrm{~b}$ & 11.80 & 4 & 3.05 & 16 & 0.319 & 22 & 14.72 & 5 & 3.08 & 16 & 0.552 & 31 & 0.870 & 38 & $10.2-18.0$ & 1971Vas \\
\hline${ }^{154} \mathrm{Gd}$ & $4 a$ & 11.97 & 4 & 2.65 & 15 & 0.304 & 19 & 15.05 & 4 & 3.35 & 14 & 0.575 & 25 & 0.879 & 32 & $10.2-18.0$ & 1971Vas \\
\hline & $4 \mathrm{~b}$ & 11.99 & 4 & 2.71 & 15 & 0.318 & 21 & 15.02 & 5 & 3.14 & 18 & 0.538 & 33 & 0.856 & 39 & $10.2-18.0$ & 1971Vas \\
\hline${ }^{156} \mathrm{Gd}$ & 0 & 12.46 & 19 & 3.14 & 74 & 0.501 & 162 & 15.79 & 32 & 4.56 & 100 & 0.683 & 201 & 1.184 & 258 & $10.9-18.7$ & $1981 \mathrm{Gur}$ \\
\hline & $4 a$ & 11.83 & 4 & 3.57 & 23 & 0.485 & 31 & 15.06 & 4 & 2.31 & 12 & 0.402 & 22 & 0.887 & 38 & $11.0-18.8$ & 1971Vas \\
\hline & $4 \mathrm{~b}$ & 11.81 & 5 & 3.90 & 35 & 0.526 & 50 & 15.04 & 6 & 2.26 & 21 & 0.379 & 42 & 0.904 & 65 & $11.0-18.8$ & 1971Vas \\
\hline${ }^{158} \mathrm{Gd}$ & $4 a$ & 11.86 & 4 & 2.94 & 15 & 0.368 & 21 & 15.16 & 5 & 3.31 & 20 & 0.559 & 26 & 0.927 & 34 & $10.2-18.0$ & 1971Vas \\
\hline & $4 \mathrm{~b}$ & 11.92 & 4 & 3.09 & 16 & 0.415 & 25 & 15.03 & 8 & 2.44 & 27 & 0.438 & 47 & 0.853 & 53 & $10.2-18.0$ & 1971Vas \\
\hline
\end{tabular}


Table 1 (continued)

\begin{tabular}{|c|c|c|c|c|c|c|c|c|c|c|c|c|c|c|c|c|c|}
\hline \multirow{2}{*}{$\begin{array}{l}\text { Nucl } \\
{ }^{160} \mathrm{Gd}\end{array}$} & \multirow{2}{*}{$\frac{\text { Id }}{3 a}$} & \multicolumn{2}{|c|}{$\begin{array}{c}E_{r, 1} \\
(\mathrm{MeV})\end{array}$} & \multicolumn{2}{|c|}{$\begin{array}{c}\Gamma_{r, 1} \\
(\mathrm{MeV})\end{array}$} & \multicolumn{2}{|l|}{$s_{1}$} & \multicolumn{2}{|c|}{$\begin{array}{c}E_{r, 2} \\
(\mathrm{MeV})\end{array}$} & $\begin{array}{l}\Gamma_{r} \\
(\mathrm{Me}\end{array}$ & & $s_{2}$ & & $s$ & & $\begin{array}{c}\varepsilon_{\min }-\varepsilon_{\max } \\
(\mathrm{MeV})\end{array}$ & Ref \\
\hline & & 12.28 & 10 & 3.33 & 42 & 0.532 & 83 & 16.06 & 15 & 5.12 & 42 & 0.863 & 97 & 1.395 & 128 & $10.9-18.8$ & 2003Var \\
\hline & $3 \mathrm{~b}$ & 12.26 & 12 & 3.27 & 60 & 0.513 & 123 & 16.03 & 20 & 5.32 & 79 & 0.897 & 167 & 1.410 & 207 & $10.9-18.8$ & 2003Var \\
\hline & 4 & 12.23 & 5 & 2.78 & 23 & 0.409 & 38 & 15.95 & 8 & 5.23 & 26 & 0.822 & 50 & 1.231 & 63 & $10.9-18.7$ & 1969Be8 \\
\hline${ }^{159} \mathrm{~Tb}$ & 4 & 12.42 & 4 & 2.71 & 14 & 0.317 & 28 & 15.86 & 6 & 5.98 & 20 & 1.205 & 46 & 1.522 & 54 & $11.1-19.0$ & 1976Gor \\
\hline & 4 & 12.08 & 5 & 2.97 & 25 & 0.397 & 40 & 15.87 & 7 & 5.06 & 27 & 0.848 & 53 & 1.245 & 66 & $11.0-18.7$ & 1968Be5 \\
\hline & 4 & 12.22 & 4 & 2.65 & 17 & 0.327 & 28 & 15.66 & 7 & 4.91 & 28 & 0.731 & 45 & 1.058 & 53 & $10.8-18.7$ & $1964 \mathrm{Br} 1$ \\
\hline & $4 a$ & 12.57 & 8 & 3.75 & 26 & 0.775 & 51 & 16.27 & 10 & 1.80 & 19 & 0.385 & 36 & 1.159 & 62 & $10.8-18.8$ & 1962Bog \\
\hline & $4 \mathrm{~b}$ & 12.36 & 8 & 3.30 & 33 & 0.622 & 63 & 16.08 & 12 & 3.05 & 30 & 0.610 & 59 & 1.232 & 86 & $10.8-18.8$ & 1962Bog \\
\hline & $4 a$ & 12.35 & 6 & 2.79 & 21 & 0.447 & 43 & 16.26 & 10 & 4.71 & 45 & 0.916 & 85 & 1.363 & 95 & $11.0-18.0$ & $1958 \mathrm{Ful}$ \\
\hline & $4 \mathrm{~b}$ & 12.34 & 6 & 2.79 & 21 & 0.446 & 44 & 16.26 & 11 & 4.72 & 46 & 0.919 & 87 & 1.365 & 97 & $11.0-18.0$ & 1958Ful \\
\hline & 5 & 12.00 & 4 & 3.01 & 25 & 0.326 & 40 & 15.40 & 10 & 6.09 & 26 & 0.913 & 55 & 1.239 & 68 & $11.1-19.0$ & 2011Var \\
\hline${ }^{165} \mathrm{Ho}$ & 0 & 12.38 & 11 & 2.59 & 56 & 0.376 & 94 & 15.48 & 21 & 4.05 & 70 & 0.604 & 122 & 0.980 & 154 & $11.1-18.7$ & $1981 \mathrm{Gur}$ \\
\hline & 0 & 12.51 & 11 & 2.72 & 53 & 0.416 & 97 & 15.37 & 18 & 3.75 & 60 & 0.593 & 118 & 1.009 & 153 & $11.1-18.7$ & 1976Gu2 \\
\hline & 4 & 12.28 & 2 & 2.58 & 9 & 0.365 & 16 & 15.78 & 3 & 4.94 & 13 & 0.793 & 23 & 1.158 & 28 & $10.9-18.7$ & 1969Be8 \\
\hline & 4 & 12.01 & 3 & 2.50 & 17 & 0.394 & 34 & 15.58 & 7 & 5.08 & 22 & 0.968 & 47 & 1.362 & 58 & $11.0-18.7$ & 1968Be5 \\
\hline & 4 & 11.84 & 3 & 2.37 & 14 & 0.359 & 25 & 16.15 & 4 & 5.70 & 22 & 1.381 & 51 & 1.740 & 57 & $11.0-18.3$ & 1966Axe \\
\hline & $4 a$ & 12.31 & 3 & 2.52 & 14 & 0.307 & 26 & 16.19 & 6 & 6.28 & 27 & 1.255 & 56 & 1.563 & 62 & $10.8-18.8$ & 1976Gor \\
\hline & $4 \mathrm{~b}$ & 12.31 & 3 & 2.52 & 14 & 0.307 & 26 & 16.19 & 6 & 6.28 & 27 & 1.255 & 56 & 1.563 & 62 & $10.8-18.8$ & 1976Gor \\
\hline${ }^{168} \mathrm{Er}$ & 0 & 12.09 & 25 & 3.66 & 129 & 0.562 & 242 & 15.54 & 27 & 3.99 & 85 & 0.652 & 221 & 1.214 & 328 & $10.9-18.8$ & 1981Gur \\
\hline${ }^{174} \mathrm{Yb}$ & 0 & 12.50 & 19 & 3.41 & 65 & 0.724 & 189 & 15.68 & 25 & 3.74 & 72 & 0.683 & 196 & 1.407 & 272 & $10.9-18.7$ & $1981 \mathrm{Gur}$ \\
\hline${ }^{175} \mathrm{Lu}$ & 4 & 12.32 & 6 & 2.59 & 28 & 0.351 & 50 & 15.47 & 10 & 4.64 & 31 & 0.820 & 68 & 1.171 & 84 & $11.0-18.7$ & 1969Be6 \\
\hline${ }^{176} \mathrm{Hf}$ & 4 & 12.34 & 3 & 2.77 & 13 & 0.476 & 28 & 15.67 & 6 & 4.72 & 17 & 0.799 & 38 & 1.275 & 47 & $10.9-17.9$ & 1977Gor \\
\hline${ }^{178} \mathrm{Hf}$ & 0 & 12.42 & 21 & 4.89 & 76 & 1.086 & 208 & 15.70 & 19 & 3.13 & 61 & 0.449 & 150 & 1.535 & 256 & $10.8-18.6$ & 1981Gur \\
\hline & 0 & 12.20 & 14 & 3.29 & 33 & 0.681 & 105 & 15.42 & 19 & 3.88 & 48 & 0.739 & 124 & 1.419 & 162 & $8.1-18.6$ & $1976 \mathrm{Gu} 2$ \\
\hline & 4 & 12.44 & 4 & 2.89 & 15 & 0.534 & 34 & 15.78 & 6 & 4.05 & 18 & 0.683 & 40 & 1.217 & 52 & $11.0-17.9$ & 1977Gor \\
\hline & 4 & 13.35 & 24 & 4.24 & 50 & 0.763 & 206 & 16.35 & 49 & 4.74 & 121 & 0.441 & 229 & 1.203 & 308 & $11.0-18.8$ & 1976Gor \\
\hline${ }^{180} \mathrm{Hf}$ & 0 & 12.55 & 30 & 4.71 & 100 & 1.004 & 281 & 15.61 & 23 & 3.27 & 80 & 0.482 & 217 & 1.486 & 355 & $10.8-18.7$ & 1981Gur \\
\hline & 0 & 12.29 & 16 & 3.08 & 37 & 0.601 & 117 & 15.33 & 21 & 3.97 & 55 & 0.785 & 146 & 1.386 & 187 & $8.6-18.7$ & $1976 \mathrm{Gu} 2$ \\
\hline & 4 & 12.46 & 3 & 2.68 & 11 & 0.498 & 23 & 15.75 & 4 & 3.78 & 13 & 0.654 & 26 & 1.152 & 35 & $11.0-17.9$ & 1977Gor \\
\hline${ }^{181} \mathrm{Ta}$ & 0 & 12.19 & 29 & 2.93 & 113 & 0.462 & 297 & 14.99 & 53 & 5.13 & 88 & 0.979 & 347 & 1.441 & 457 & $10.8-18.6$ & 1981Gur \\
\hline & 0 & 12.19 & 29 & 2.93 & 113 & 0.462 & 297 & 14.99 & 53 & 5.13 & 88 & 0.979 & 346 & 1.441 & 456 & $10.8-18.6$ & $1976 \mathrm{Gu} 2$ \\
\hline & $3 a$ & 12.30 & 8 & 2.44 & 22 & 0.372 & 52 & 15.20 & 12 & 4.51 & 23 & 0.918 & 66 & 1.290 & 84 & $10.0-18.8$ & 2003Var \\
\hline & $3 \mathrm{~b}$ & 12.32 & 13 & 2.56 & 48 & 0.388 & 110 & 15.23 & 20 & 4.54 & 66 & 0.907 & 165 & 1.295 & 198 & $10.0-18.8$ & 2003Var \\
\hline & 4 & 12.54 & 1 & 2.55 & 4 & 0.447 & 11 & 15.48 & 2 & 3.85 & 7 & 0.769 & 15 & 1.217 & 18 & $10.8-18.8$ & 2001Bel \\
\hline & 4 & 12.31 & 5 & 2.50 & 20 & 0.392 & 42 & 15.24 & 7 & 4.41 & 20 & 0.892 & 53 & 1.284 & 68 & $11.0-18.7$ & 1968Be5 \\
\hline & 4 & 12.54 & 5 & 1.75 & 23 & 0.156 & 36 & 14.89 & 14 & 5.03 & 34 & 0.839 & 67 & 0.995 & 76 & $10.8-18.7$ & 1963Br1 \\
\hline & 4 & 12.67 & 10 & 3.33 & 46 & 0.800 & 104 & 15.70 & 5 & 2.55 & 20 & 0.577 & 75 & 1.376 & 128 & $11.0-18.5$ & 1962Bog \\
\hline & $4 a$ & 11.85 & 6 & 2.35 & 26 & 0.358 & 41 & 14.99 & 8 & 4.27 & 20 & 0.768 & 42 & 1.126 & 59 & $10.8-18.8$ & 1969Ish \\
\hline & $4 \mathrm{~b}$ & 11.94 & 8 & 2.47 & 33 & 0.419 & 56 & 15.11 & 8 & 3.42 & 28 & 0.648 & 57 & 1.066 & 80 & $10.8-18.8$ & 1969Ish \\
\hline & $4 a$ & 12.29 & 23 & 3.10 & 84 & 0.512 & 152 & 15.57 & 28 & 3.59 & 67 & 0.638 & 158 & 1.149 & 219 & $11.0-18.0$ & 1967Ant \\
\hline & $4 \mathrm{~b}$ & 12.23 & 29 & 2.95 & 127 & 0.465 & 241 & 15.49 & 42 & 3.96 & 142 & 0.703 & 291 & 1.168 & 377 & $11.0-18.0$ & 1967Ant \\
\hline & $4 a$ & 12.33 & 2 & 2.34 & 12 & 0.412 & 28 & 15.46 & 6 & 5.07 & 18 & 1.091 & 46 & 1.503 & 53 & $11.0-18.7$ & $1958 \mathrm{Ful}$ \\
\hline & $4 \mathrm{~b}$ & 12.33 & 2 & 2.40 & 13 & 0.426 & 30 & 15.47 & 6 & 5.02 & 20 & 1.074 & 49 & 1.500 & 57 & -18.7 & $1958 \mathrm{Ful}$ \\
\hline & 5 & 12.38 & 17 & 2.86 & 82 & 0.441 & 168 & 15.19 & 29 & 4.83 & 37 & 0.824 & 168 & 1.265 & 237 & $11.5-19.0$ & 2012 Var \\
\hline${ }^{182} \mathrm{~W}$ & 0 & 11.98 & 37 & 3.91 & 199 & 0.662 & 515 & 14.94 & 61 & 5.16 & 136 & 0.798 & 497 & 1.460 & 716 & $11.0-18.8$ & 1981Gur \\
\hline & 0 & 11.90 & 18 & 2.58 & 51 & 0.403 & 138 & 14.71 & 32 & 5.17 & 66 & 0.986 & 191 & 1.389 & 235 & $8.5-18.8$ & 1976Gu2 \\
\hline & 4 & 12.64 & 4 & 2.60 & 11 & 0.446 & 31 & 15.45 & 7 & 4.66 & 13 & 0.916 & 41 & 1.361 & 51 & $10.8-18.6$ & 1978Go3 \\
\hline${ }^{184} \mathrm{~W}$ & 0 & 11.92 & 27 & 4.52 & 167 & 0.930 & 432 & 15.05 & 29 & 3.87 & 110 & 0.534 & 319 & 1.464 & 537 & $11.0-17.6$ & 1981Gur \\
\hline & 4 & 12.44 & 5 & 2.24 & 19 & 0.304 & 42 & 15.02 & 8 & 5.11 & 12 & 1.121 & 54 & 1.425 & 68 & $8.0-18.6$ & 1978Go3 \\
\hline & $4 a$ & 12.39 & 6 & 2.53 & 31 & 0.338 & 87 & 15.52 & 22 & 6.14 & 72 & 1.245 & 160 & 1.583 & 182 & $11.0-18.8$ & 1973Gor \\
\hline & $4 \mathrm{~b}$ & 12.43 & 8 & 2.76 & 39 & 0.417 & 138 & 15.68 & 21 & 5.40 & 157 & 1.098 & 324 & 1.515 & 352 & $11.0-18.8$ & 1973Gor \\
\hline${ }^{186} \mathrm{~W}$ & 0 & 13.04 & 30 & 6.60 & 56 & 1.591 & 202 & 14.89 & 41 & 2.12 & 202 & 0.086 & 134 & 1.677 & 242 & $10.9-18.7$ & 1981Gur \\
\hline & 4 & 12.58 & 5 & 2.53 & 16 & 0.379 & 42 & 15.07 & 8 & 4.72 & 15 & 0.988 & 55 & 1.367 & 69 & $11.0-17.8$ & 1978Go3 \\
\hline & 4 & 12.59 & 3 & 2.32 & 14 & 0.292 & 34 & 14.89 & 8 & 5.10 & 14 & 0.989 & 48 & 1.281 & 59 & $10.9-18.7$ & 1969Be8 \\
\hline & $4 a$ & 12.64 & 9 & 3.11 & 32 & 0.496 & 114 & 15.55 & 30 & 5.61 & 60 & 0.930 & 157 & 1.427 & 193 & $11.0-18.8$ & 1973Gor \\
\hline & $4 \mathrm{~b}$ & 12.65 & 11 & 3.17 & 38 & 0.528 & 158 & 15.60 & 35 & 5.23 & 142 & 0.869 & 279 & 1.397 & 321 & $11.0-18.8$ & 1973Gor \\
\hline${ }^{185} \mathrm{Re}$ & $4 a$ & 12.59 & 4 & 2.22 & 24 & 0.289 & 59 & 15.17 & 16 & 6.02 & 37 & 1.162 & 99 & 1.451 & 115 & $10.2-18.0$ & 1973Gor \\
\hline & $4 \mathrm{~b}$ & 12.60 & 3 & 2.19 & 21 & 0.284 & 50 & 15.27 & 22 & 6.22 & 37 & 1.222 & 102 & 1.506 & 113 & $10.2-18.0$ & 1973Gor \\
\hline${ }^{\text {nat }} \operatorname{Re}$ & 4 & 14.11 & 1 & 6.52 & 4 & 1.787 & 9 & & & & & & & 1.787 & 9 & $10.2-18.0$ & 1975Vey \\
\hline${ }^{186} \mathrm{Os}$ & 5 & 12.73 & 36 & 2.34 & 93 & 0.292 & 218 & 14.73 & 38 & 4.08 & 31 & 0.845 & 237 & 1.137 & 322 & $11.1-18.9$ & 2015Var \\
\hline & 7 & 13.04 & 11 & 3.14 & 28 & 0.570 & 91 & 15.27 & 12 & 3.33 & 27 & 0.575 & 93 & 1.145 & 130 & $11.1-18.7$ & 1979Be4 \\
\hline${ }^{188} \mathrm{Os}$ & 5 & 12.81 & 39 & 2.83 & 85 & 0.460 & 277 & 14.93 & 38 & 3.83 & 45 & 0.861 & 291 & 1.321 & 402 & $10.8-18.9$ & 2014Var \\
\hline & 9 & 12.81 & 6 & 2.77 & 15 & 0.419 & 55 & 14.88 & 8 & 4.15 & 15 & 0.929 & 67 & 1.348 & 87 & $10.8-18.7$ & 1979Be4 \\
\hline${ }^{189} \mathrm{Os}$ & 5 & 12.92 & 31 & 3.07 & 64 & 0.582 & 253 & 15.02 & 38 & 3.94 & 38 & 0.680 & 254 & 1.262 & 359 & $10.8-18.9$ & 2014Var \\
\hline & 9 & 12.64 & 6 & 2.60 & 14 & 0.373 & 48 & 14.63 & 7 & 3.78 & 14 & 0.884 & 60 & 1.257 & 77 & $10.8-18.7$ & 1979Be4 \\
\hline${ }^{190} \mathrm{Os}$ & 5 & 13.10 & 50 & 3.34 & 89 & 0.715 & 442 & 15.12 & 65 & 3.80 & 67 & 0.541 & 434 & 1.255 & 620 & $10.8-18.9$ & 2015Var \\
\hline & 9 & 12.64 & 9 & 2.53 & 25 & 0.283 & 76 & 14.36 & 11 & 4.17 & 13 & 0.981 & 90 & 1.264 & 118 & $10.8-18.7$ & 1979Be4 \\
\hline
\end{tabular}


Table 1 (continued)

\begin{tabular}{|c|c|c|c|c|c|c|c|c|c|c|c|c|c|c|c|c|c|}
\hline \multirow{2}{*}{$\begin{array}{c}\text { Nucl } \\
{ }^{192} \mathrm{Os}\end{array}$} & \multirow{2}{*}{$\frac{\text { Id }}{5}$} & \multicolumn{2}{|c|}{$\begin{array}{c}E_{r, 1} \\
(\mathrm{MeV})\end{array}$} & \multicolumn{2}{|c|}{$\begin{array}{c}\Gamma_{r, 1} \\
(\mathrm{MeV}) \\
\end{array}$} & \multicolumn{2}{|l|}{$s_{1}$} & \multicolumn{2}{|c|}{$\begin{array}{c}E_{r, 2} \\
(\mathrm{MeV}) \\
\end{array}$} & $\begin{array}{r}\Gamma_{r} \\
(\mathrm{Me}\end{array}$ & & $s_{2}$ & & $s$ & & $\begin{array}{c}\varepsilon_{\min }-\varepsilon_{\max } \\
(\mathrm{MeV})\end{array}$ & Ref \\
\hline & & 12.59 & 41 & 2.13 & 134 & 0.211 & 276 & 14.32 & 46 & 4.60 & 29 & 1.118 & 313 & 1.329 & 417 & $10.8-18.9$ & 2015Var \\
\hline & 9 & 12.64 & 9 & 2.53 & 25 & 0.281 & 76 & 14.36 & 11 & 4.17 & 13 & 0.974 & 89 & 1.255 & 117 & $10.8-18.7$ & 1979Be4 \\
\hline${ }^{191} \mathrm{Ir}$ & 4 & 12.72 & 10 & 2.08 & 73 & 0.217 & 167 & 14.21 & 32 & 5.27 & 27 & 1.148 & 208 & 1.365 & 267 & $11.0-16.8$ & $1978 \mathrm{Go} 1$ \\
\hline & 4 & 12.98 & 10 & 2.91 & 41 & 0.591 & 196 & 15.98 & 15 & 4.91 & 171 & 1.009 & 421 & 1.600 & 465 & $11.1-16.9$ & 1978Go2 \\
\hline${ }^{193} \mathrm{Ir}$ & 4 & 12.86 & 6 & 1.90 & 37 & 0.247 & 92 & 14.30 & 22 & 5.62 & 29 & 1.132 & 110 & 1.379 & 143 & $11.0-16.8$ & $1978 \mathrm{Go} 1$ \\
\hline $\mathrm{nat}^{\mathrm{Ir}}$ & 4 & 13.77 & 1 & 4.86 & 4 & 1.362 & 7 & & & & & & & 1.362 & 7 & $10.2-18.0$ & 1975Vey \\
\hline${ }^{194} \mathrm{Pt}$ & 4 & 13.42 & 7 & 3.61 & 20 & 0.918 & 128 & 15.97 & 63 & 6.16 & 95 & 0.386 & 159 & 1.304 & 204 & $11.0-17.8$ & $1978 \mathrm{Go} 1$ \\
\hline & 13 & 13.22 & 5 & 3.23 & 16 & 0.750 & 76 & 16.30 & 13 & 5.15 & 51 & 0.821 & 114 & 1.571 & 136 & $11.2-18.0$ & 1978Go2 \\
\hline${ }^{195} \mathrm{Pt}$ & 4 & 12.99 & 15 & 2.92 & 49 & 0.584 & 286 & 14.90 & 66 & 4.85 & 91 & 0.689 & 355 & 1.273 & 456 & $11.0-17.8$ & $1978 \mathrm{Go} 1$ \\
\hline & 13 & 13.06 & 9 & 2.79 & 40 & 0.573 & 193 & 15.85 & 35 & 5.63 & 136 & 1.057 & 345 & 1.630 & 395 & $11.2-18.0$ & 1978Go2 \\
\hline${ }^{196} \mathrm{Pt}$ & 4 & 13.28 & 4 & 3.10 & 27 & 0.597 & 126 & 14.81 & 40 & 7.51 & 42 & 0.808 & 143 & 1.405 & 191 & $11.0-17.8$ & 1978Go1 \\
\hline${ }^{198} \mathrm{Pt}$ & 4 & 13.56 & $\begin{array}{l}4 \\
2\end{array}$ & 4.88 & 6 & 1.429 & 11 & & & & & & & 1.429 & 11 & $11.0-17.8$ & $1978 \mathrm{Go} 1$ \\
\hline${ }^{197} \mathrm{Au}$ & 0 & 13.58 & 7 & 5.32 & 28 & 1.539 & 61 & & & & & & & 1.539 & 61 & $11.1-17.0$ & 1981Gur \\
\hline & 0 & 13.57 & 6 & 4.89 & 4 & 1.448 & 180 & & & & & & & 1.448 & 41 & $8.2-17.0$ & $1976 \mathrm{Gu} 2$ \\
\hline & 4 & 13.71 & 2 & 4.51 & 7 & 1.354 & 16 & & & & & & & 1.354 & 16 & $11.0-16.8$ & 1970Ve1 \\
\hline & 4 & 13.83 & 3 & 3.84 & 8 & 1.170 & 19 & & & & & & & 1.170 & 19 & $11.1-16.8$ & 1962Fu2 \\
\hline & $4 a$ & 13.19 & 3 & 4.45 & 14 & 1.341 & 28 & & & & & & & 1.341 & 28 & $11.1-17.0$ & 1973Sor \\
\hline & $4 \mathrm{~b}$ & 13.22 & 4 & 4.70 & 21 & 1.396 & 44 & & & & & & & 1.396 & 44 & $11.1-17.0$ & 1973Sor \\
\hline & $4 a$ & 13.67 & 3 & 4.76 & 11 & 1.484 & 25 & & & & & & & 1.484 & 25 & $11.4-17.0$ & $1958 \mathrm{Ful}$ \\
\hline & $4 \mathrm{~b}$ & 13.67 & 3 & 4.82 & 12 & 1.499 & 27 & & & & & & & 1.499 & 27 & $11.4-17.0$ & $1958 \mathrm{Ful}$ \\
\hline & 8 & 13.71 & 3 & 4.88 & 14 & 1.345 & 32 & & & & & & & 1.345 & 32 & $12.1-16.9$ & 1987Ber \\
\hline & $8 \mathrm{a}$ & 13.65 & 6 & 4.13 & 20 & 1.254 & 35 & & & & & & & 1.254 & 35 & $11.0-16.8$ & 2010Var \\
\hline & $8 \mathrm{~b}$ & 13.64 & 13 & 4.25 & 42 & 1.268 & 94 & & & & & & & 1.268 & 94 & $11.0-16.8$ & 2010Var \\
\hline${ }^{203} \mathrm{Tl}$ & 4 & 14.04 & 7 & 3.77 & 3 & 0.886 & 190 & & & & & & & 0.886 & 34 & $9.0-17.9$ & 1970Ant \\
\hline${ }^{205} \mathrm{Tl}$ & 4 & 14.46 & 5 & 2.95 & 15 & 0.755 & 25 & & & & & & & 0.755 & 25 & $10.5-17.9$ & 1970Ant \\
\hline${ }^{206} \mathrm{~Pb}$ & 4 & 13.58 & 1 & 3.83 & 5 & 1.041 & 8 & & & & & & & 1.041 & 8 & $10.0-17.0$ & $1964 \mathrm{Ha} 2$ \\
\hline & $4 a$ & 13.44 & 2 & 4.87 & 5 & 1.171 & 10 & & & & & & & 1.171 & 10 & $10.1-16.9$ & 1973Sor \\
\hline & $4 \mathrm{~b}$ & 13.54 & 2 & 5.16 & 7 & 1.240 & 15 & & & & & & & 1.240 & 15 & $10.1-16.9$ & 1973Sor \\
\hline${ }^{207} \mathrm{~Pb}$ & 4 & 13.55 & 2 & 3.95 & 5 & 1.002 & 8 & & & & & & & 1.002 & 8 & $10.0-17.0$ & $1964 \mathrm{Ha} 2$ \\
\hline${ }^{208} \mathrm{~Pb}$ & $3 a$ & 13.37 & 3 & 3.93 & 8 & 1.337 & 16 & & & & & & & 1.337 & 16 & $10.9-18.8$ & 2003Var \\
\hline & $3 \mathrm{~b}$ & 13.41 & 7 & 3.97 & 19 & 1.342 & 45 & & & & & & & 1.342 & 45 & $10.9-18.8$ & 2003Var \\
\hline & 4 & 13.46 & 1 & 3.58 & 2 & 1.121 & 6 & & & & & & & 1.121 & 6 & $10.8-18.8$ & 1995Bel \\
\hline & 4 & 13.42 & 2 & 4.14 & 4 & 1.368 & 11 & & & & & & & 1.368 & 11 & $10.2-16.8$ & 1970Ve1 \\
\hline & 4 & 13.45 & 1 & 3.89 & 4 & 1.004 & 7 & & & & & & & 1.004 & 7 & $10.0-17.0$ & $1964 \mathrm{Ha} 2$ \\
\hline & $4 a$ & 13.18 & 1 & 4.34 & 4 & 1.139 & 9 & & & & & & & 1.139 & 9 & $10.9-18.6$ & 1973Sor \\
\hline & $4 \mathrm{~b}$ & 13.36 & 2 & 5.01 & 8 & 1.306 & 19 & & & & & & & 1.306 & 19 & $10.9-18.6$ & 1973Sor \\
\hline & 5 & 13.38 & 3 & 4.08 & 9 & 1.347 & 20 & & & & & & & 1.347 & 20 & $10.5-17.1$ & 2013Ish \\
\hline & 12 & 13.63 & 2 & 3.93 & 5 & 1.334 & 13 & & & & & & & 1.334 & 13 & $10.0-14.9$ & $1972 \mathrm{You}$ \\
\hline${ }^{\text {nat }} \mathrm{Pb}$ & 2 & 13.48 & 2 & 3.99 & 7 & 1.341 & 18 & & & & & & & 1.341 & 18 & $12.1-16.9$ & 1985Ahr \\
\hline & 8 & 13.57 & 2 & 3.78 & 9 & 1.227 & 24 & & & & & & & 1.227 & 24 & $12.1-16.9$ & 1987Ber \\
\hline${ }^{209} \mathrm{Bi}$ & 0 & 13.79 & 8 & 5.02 & 29 & 1.546 & 64 & & & & & & & 1.546 & 64 & $10.9-18.3$ & 1976Gu2 \\
\hline & 4 & 13.32 & 1 & 3.76 & 2 & 1.030 & 4 & & & & & & & 1.030 & 4 & $10.8-18.8$ & 1995Bel \\
\hline & 4 & 13.44 & 1 & 3.96 & 4 & 1.077 & 6 & & & & & & & 1.077 & 6 & $10.0-17.0$ & $1964 \mathrm{Ha} 2$ \\
\hline & $4 a$ & 13.03 & 4 & 4.12 & 10 & 1.266 & 20 & & & & & & & 1.266 & 20 & $10.9-18.6$ & 1973Sor \\
\hline & $4 \mathrm{~b}$ & 13.10 & 4 & 4.53 & 17 & 1.372 & 39 & & & & & & & 1.372 & 39 & $10.9-18.6$ & 1973Sor \\
\hline & $4 a$ & 12.93 & 9 & 3.59 & 16 & 1.306 & 37 & & & & & & & 1.306 & 37 & $11.0-18.5$ & 1967Ant \\
\hline & $4 \mathrm{~b}$ & 13.05 & 19 & 3.82 & 33 & 1.327 & 93 & & & & & & & 1.327 & 93 & $11.0-18.5$ & 1967Ant \\
\hline & 5 & 13.56 & 6 & 4.22 & 18 & 1.131 & 28 & & & & & & & 1.131 & 28 & $10.0-18.9$ & 2016Va2 \\
\hline & 12 & 13.56 & 1 & 3.72 & 4 & 1.259 & 10 & & & & & & & 1.259 & 10 & $10.0-14.8$ & $1972 \mathrm{You}$ \\
\hline${ }^{232} \mathrm{Th}$ & 0 & 10.86 & 19 & 2.86 & 122 & 0.313 & 182 & 13.74 & 27 & 4.74 & 54 & 0.861 & 183 & 1.174 & 258 & $10.2-18.3$ & 1976Gu1 \\
\hline & 4 & 11.09 & 7 & 3.22 & 46 & 0.518 & 99 & 14.02 & 10 & 4.63 & 21 & 0.958 & 90 & 1.476 & 134 & $10.4-18.3$ & $1980 \mathrm{Ca} 1$ \\
\hline & 4 & 11.27 & 36 & 4.34 & 106 & 0.588 & 252 & 14.18 & 28 & 4.43 & 98 & 0.636 & 264 & 1.224 & 365 & $9.2-16.3$ & 1973Ve1 \\
\hline & 5 & 11.03 & 13 & 3.13 & 58 & 0.369 & 107 & 13.92 & 19 & 4.73 & 42 & 0.777 & 116 & 1.146 & 157 & $10.0-18.1$ & 2007Var \\
\hline & 10 & 11.04 & 2 & 2.71 & 7 & 0.394 & 23 & 13.87 & 4 & 4.73 & 15 & 1.005 & 37 & 1.399 & 44 & $9.4-17.8$ & $1980 \mathrm{Ca} 1$ \\
\hline${ }^{233} \mathrm{U}$ & 4 & 10.98 & 4 & 1.53 & 17 & 0.101 & 20 & 13.30 & 7 & 5.71 & 12 & 1.179 & 33 & 1.279 & 39 & $9.4-17.8$ & 1986Be2 \\
\hline & 11 & 11.10 & 2 & 1.78 & 7 & 0.263 & 17 & 13.97 & 3 & 5.26 & 6 & 2.370 & 29 & 2.633 & 34 & $9.4-17.8$ & 1986Be2 \\
\hline${ }^{234} \mathrm{U}$ & 4 & 11.18 & 7 & 2.41 & 27 & 0.429 & 68 & 14.03 & 11 & 4.46 & 33 & 0.834 & 81 & 1.263 & 105 & $9.4-17.8$ & 1986Be2 \\
\hline & 11 & 11.08 & 5 & 2.20 & 19 & 0.530 & 59 & 14.23 & 5 & 4.43 & 19 & 1.775 & 79 & 2.305 & 99 & $9.4-17.8$ & 1986Be2 \\
\hline${ }^{235} \mathrm{U}$ & 0 & 10.82 & 22 & 3.88 & 80 & 0.548 & 161 & 13.80 & 23 & 4.54 & 58 & 0.678 & 159 & 1.226 & 226 & $9.5-18.4$ & 1976Gu1 \\
\hline & 4 & 10.95 & 4 & 2.30 & 14 & 0.356 & 30 & 14.04 & 6 & 4.79 & 21 & 1.024 & 49 & 1.380 & 58 & $9.4-18.3$ & $1980 \mathrm{Ca} 1$ \\
\hline${ }^{236} \mathrm{U}$ & 4 & 11.04 & 5 & 2.65 & 18 & 0.346 & 40 & 13.92 & 8 & 4.77 & 20 & 0.906 & 52 & 1.252 & 66 & $9.5-17.8$ & $1980 \mathrm{Ca} 1$ \\
\hline & 10 & 10.93 & 3 & 2.58 & 11 & 0.335 & 24 & 13.80 & 6 & 4.78 & 14 & 0.915 & 35 & 1.250 & 42 & $9.4-17.8$ & $1980 \mathrm{Ca} 1$ \\
\hline${ }^{238} \mathrm{U}$ & 0 & 11.06 & 13 & 2.95 & 48 & 0.388 & 84 & 14.26 & 19 & 4.80 & 51 & 0.773 & 106 & 1.161 & 136 & $9.2-18.8$ & 1976Gu1 \\
\hline & 4 & 10.83 & 3 & 2.95 & 12 & 0.466 & 29 & 13.96 & 5 & 4.90 & 16 & 0.978 & 40 & 1.445 & 50 & $9.0-18.3$ & $1980 \mathrm{Ca} 1$ \\
\hline & 4 & 10.94 & 4 & 2.64 & 14 & 0.364 & 28 & 13.99 & 6 & 4.56 & 18 & 0.803 & 40 & 1.167 & 49 & $9.1-17.8$ & 1973Ve1 \\
\hline & 5 & 10.88 & 7 & 2.54 & 23 & 0.299 & 45 & 13.90 & 13 & 4.96 & 31 & 0.825 & 64 & 1.124 & 70 & $9.2-18.2$ & 2007Var \\
\hline${ }^{n a t} U$ & 2 & 10.73 & 2 & 2.47 & 13 & 0.345 & 24 & 13.72 & 4 & 5.04 & 9 & 0.949 & 27 & 1.294 & 36 & $10.2-17.9$ & $1985 \mathrm{Ahr}$ \\
\hline
\end{tabular}


Table 1 (continued)

\begin{tabular}{|c|c|c|c|c|c|c|c|c|c|c|c|c|c|c|}
\hline Nucl & Id & $\begin{array}{c}E_{r, 1} \\
(\mathrm{MeV})\end{array}$ & $\begin{array}{c}\Gamma_{r, 1} \\
(\mathrm{MeV})\end{array}$ & $s_{1}$ & & $\begin{array}{r}E_{r, 2} \\
(\mathrm{MeV}\end{array}$ & & $\begin{array}{c}\Gamma_{r, 2} \\
(\mathrm{MeV})\end{array}$ & $s_{2}$ & & $s$ & & $\begin{array}{c}\varepsilon_{\min }-\varepsilon_{\max } \\
(\mathrm{MeV})\end{array}$ & Ref \\
\hline \multirow[t]{5}{*}{${ }^{237} \mathrm{~Np}$} & 4 & 10.994 & $2.20 \quad 12$ & 0.316 & 27 & 14.08 & 6 & $4.66 \quad 18$ & 1.157 & 43 & 1.473 & 50 & $9.4-17.8$ & $1986 \mathrm{Be} 2$ \\
\hline & 4 & $11.01 \quad 15$ & $2.91 \quad 63$ & 0.347 & 108 & 14.05 & 18 & $4.52 \quad 58$ & 0.820 & 137 & 1.167 & 174 & $9.5-16.5$ & 1973Ve1 \\
\hline & 5 & $10.89 \quad 10$ & $2.28 \quad 31$ & 0.250 & 55 & 13.98 & 16 & $4.71 \quad 43$ & 0.905 & 86 & 1.156 & 102 & $9.3-17.7$ & 2007Var \\
\hline & 10 & $10.98 \quad 5$ & $2.17 \quad 16$ & 0.312 & 38 & 14.06 & 9 & $4.64 \quad 27$ & 1.160 & 65 & 1.472 & 75 & $9.4-17.8$ & 1986Be2 \\
\hline & 10 & $11.01 \quad 25$ & $2.92 \quad 89$ & 0.343 & 170 & 14.10 & 32 & $4.76 \quad 112$ & 0.863 & 252 & 1.206 & 304 & $9.2-16.6$ & $1973 \mathrm{Ve} 1$ \\
\hline \multirow[t]{3}{*}{${ }^{239} \mathrm{Pu}$} & 0 & $11.07 \quad 28$ & $3.29 \quad 100$ & 0.344 & 185 & 14.00 & 38 & $5.51 \quad 74$ & 0.906 & 227 & 1.250 & 293 & $9.3-18.7$ & 1976Gu1 \\
\hline & 5 & $11.27 \quad 10$ & $2.75 \quad 27$ & 0.380 & 80 & 13.68 & 19 & $4.24 \quad 46$ & 0.658 & 107 & 1.038 & 134 & $9.2-17.7$ & 2007Var \\
\hline & 10 & $11.31 \quad 10$ & $2.48 \quad 21$ & 0.385 & 76 & 13.90 & 22 & $4.36 \quad 43$ & 0.766 & 105 & 1.151 & 130 & $9.1-17.8$ & 1986Be2 \\
\hline
\end{tabular}


Table 2

Experimental values and uncertainties of GDR parameters within Modified Lorentzian (SMLO) approach.

\begin{tabular}{|c|c|c|c|c|c|c|c|c|c|c|c|c|c|c|c|c|c|}
\hline \multirow{2}{*}{$\begin{array}{l}\text { Nucl } \\
{ }^{6} \mathrm{Li}\end{array}$} & \multirow{2}{*}{$\begin{array}{l}\text { Id } \\
2 \\
\end{array}$} & \multicolumn{2}{|c|}{$\begin{array}{c}E_{r, 1} \\
(\mathrm{MeV})\end{array}$} & \multicolumn{2}{|c|}{$\begin{array}{c}\Gamma_{r, 1} \\
(\mathrm{MeV})\end{array}$} & \multicolumn{2}{|l|}{$s_{1}$} & \multicolumn{2}{|c|}{$\begin{array}{c}E_{r, 2} \\
(\mathrm{MeV}) \\
\end{array}$} & \multicolumn{2}{|c|}{$\begin{array}{c}\Gamma_{r, 2} \\
(\mathrm{MeV}) \\
\end{array}$} & $s_{2}$ & & $s$ & & $\begin{array}{c}\varepsilon_{\min }-\varepsilon_{\max } \\
(\mathrm{MeV})\end{array}$ & Ref \\
\hline & & 23.75 & 17 & 5.33 & 81 & 0.344 & 33 & & & & & & & 0.344 & 33 & $21.5-27.0$ & 1986Var \\
\hline & 4 & 13.96 & 13 & 22.25 & 61 & 0.549 & 16 & & & & & & & 0.549 & 16 & $5.6-24.7$ & 1965Be1 \\
\hline${ }^{7} \mathrm{Li}$ & 0 & 20.21 & 49 & 21.81 & 251 & 1.155 & 122 & & & & & & & 1.155 & 122 & $13.2-25.6$ & $1985 \mathrm{Ahr}$ \\
\hline & $2 \mathrm{a}$ & 18.39 & 18 & 22.52 & 104 & 1.124 & 53 & & & & & & & 1.124 & 53 & $13.5-25.0$ & 1986Var \\
\hline & $2 \mathrm{~b}$ & 18.39 & 18 & 22.52 & 104 & 1.124 & 53 & & & & & & & 1.124 & 53 & $13.5-25.0$ & 1986Var \\
\hline & $4 a$ & 16.67 & 11 & 12.46 & 38 & 0.272 & 7 & & & & & & & 0.272 & 7 & $10.1-24.7$ & 1973Bra \\
\hline & $4 \mathrm{~b}$ & 16.88 & 14 & 13.02 & 63 & 0.281 & 11 & & & & & & & 0.281 & 11 & $10.1-24.7$ & 1973Bra \\
\hline${ }^{9} \mathrm{Be}$ & 0 & 24.18 & 14 & 10.86 & 45 & 0.651 & 25 & & & & & & & 0.651 & 25 & $17.5-26.0$ & 1975Ahr \\
\hline${ }^{10} \mathrm{~B}$ & $4 \mathrm{a}$ & 23.31 & 22 & 17.51 & 44 & 0.755 & 25 & & & & & & & 0.755 & 25 & $8.5-24.9$ & 1987Ahs \\
\hline & $4 \mathrm{~b}$ & 24.96 & 92 & 22.20 & 233 & 0.967 & 122 & & & & & & & 0.967 & 122 & $8.5-24.9$ & 1987Ahs \\
\hline${ }^{12} \mathrm{C}$ & 0 & 22.90 & 2 & 3.69 & 7 & 0.683 & 11 & & & & & & & 0.683 & 11 & $20.1-25.0$ & 1969Bez \\
\hline & 1 & 22.93 & 3 & 3.26 & 7 & 0.603 & 11 & & & & & & & 0.603 & 11 & $13.2-24.0$ & 1975Ahr \\
\hline & $3 a$ & 23.13 & 5 & 3.00 & 11 & 0.563 & 14 & & & & & & & 0.563 & 14 & $20.2-25.0$ & 2002Ish \\
\hline & $3 \mathrm{~b}$ & 23.10 & 7 & 3.07 & 16 & 0.566 & 23 & & & & & & & 0.566 & 23 & $20.2-25.0$ & 2002Ish \\
\hline & 4 & 22.82 & 7 & 3.71 & 31 & 0.504 & 29 & & & & & & & 0.504 & 29 & $14.0-24.9$ & 1963Bur \\
\hline${ }^{13} \mathrm{C}$ & $3 a$ & 25.02 & 21 & 10.72 & 64 & 1.012 & 40 & & & & & & & 1.012 & 40 & $14.5-29.0$ & 2002Ish \\
\hline & $3 b$ & 24.54 & 21 & 8.63 & 82 & 0.901 & 49 & & & & & & & 0.901 & 49 & $14.5-29.0$ & 2002Ish \\
\hline${ }^{14} \mathrm{C}$ & $3 a$ & 15.69 & 22 & 6.51 & 114 & 0.381 & 55 & 26.27 & 16 & 7.11 & 104 & 0.420 & 53 & 0.801 & 76 & $14.5-30.0$ & 2002Ish \\
\hline & $3 \mathrm{~b}$ & 16.17 & 40 & 3.80 & 62 & 0.251 & 27 & 26.07 & 19 & 7.05 & 117 & 0.475 & 61 & 0.726 & 67 & $9.0-30.0$ & 2002Ish \\
\hline${ }^{n a t} \mathrm{C}$ & 0 & 23.14 & 2 & 4.24 & 6 & 0.717 & 8 & & & & & & & 0.717 & 8 & $19.5-25.6$ & $1985 \mathrm{Ahr}$ \\
\hline & 0 & 23.40 & 1 & 4.24 & 4 & 0.740 & 5 & & & & & & & 0.740 & 5 & $19.0-26.0$ & 1975Ahr \\
\hline & 0 & 23.16 & 2 & 4.10 & 7 & 0.689 & 8 & & & & & & & 0.689 & 8 & $19.1-25.8$ & 1972Ahr \\
\hline & $1 \mathrm{a}$ & 23.53 & 10 & 2.63 & 16 & 0.467 & 14 & & & & & & & 0.467 & 14 & $19.1-26.0$ & $1965 \mathrm{Wyc}$ \\
\hline & $1 b$ & 23.92 & 21 & 3.45 & 37 & 0.507 & 28 & & & & & & & 0.507 & 28 & $19.1-26.0$ & $1965 \mathrm{Wyc}$ \\
\hline${ }^{14} \mathrm{~N}$ & 0 & 23.19 & 3 & 7.09 & 14 & 1.217 & 18 & & & & & & & 1.217 & 18 & $18.2-28.0$ & 1969Bez \\
\hline & $3 a$ & 23.36 & 9 & 5.68 & 20 & 0.808 & 18 & & & & & & & 0.808 & 18 & $15.0-28.0$ & 2002Ish \\
\hline & $3 \mathrm{~b}$ & 23.29 & 21 & 5.88 & 45 & 0.809 & 58 & & & & & & & 0.809 & 58 & $15.0-28.0$ & 2002Ish \\
\hline${ }^{15} \mathrm{~N}$ & $3 a$ & 26.29 & 57 & 19.45 & 144 & 1.752 & 149 & & & & & & & 1.752 & 149 & $14.5-28.0$ & 2002Ish \\
\hline & $3 b$ & 26.35 & 90 & 18.54 & 287 & 1.756 & 277 & & & & & & & 1.756 & 277 & $14.5-28.0$ & 2002Ish \\
\hline${ }^{16} \mathrm{O}$ & 0 & 23.78 & 4 & 5.67 & 13 & 1.028 & 19 & & & & & & & 1.028 & 19 & $18.1-26.0$ & 1975Ahr \\
\hline & 0 & 23.74 & 4 & 5.71 & 13 & 1.026 & 19 & & & & & & & 1.026 & 19 & $18.2-25.9$ & $1972 \mathrm{Ahr}$ \\
\hline & 0 & 23.45 & 5 & 5.72 & 15 & 0.983 & 22 & & & & & & & 0.983 & 22 & $18.5-26.0$ & 1969Bez \\
\hline & 0 & 22.96 & 5 & 4.70 & 17 & 1.021 & 28 & & & & & & & 1.021 & 28 & $18.9-25.9$ & 1963Bur \\
\hline & $1 \mathrm{a}$ & 23.88 & 10 & 2.79 & 12 & 0.477 & 17 & & & & & & & 0.477 & 17 & $18.2-25.6$ & $1965 \mathrm{Wyc}$ \\
\hline & $1 b$ & 24.00 & 14 & 3.13 & 20 & 0.503 & 24 & & & & & & & 0.503 & 24 & $18.2-25.6$ & 1965 Wyc \\
\hline & $3 a$ & 23.72 & 8 & 4.36 & 14 & 0.822 & 20 & & & & & & & 0.822 & 20 & $18.2-26.0$ & 2002Ish \\
\hline & $3 \mathrm{~b}$ & 23.91 & 13 & 4.98 & 26 & 0.882 & 35 & & & & & & & 0.882 & 35 & $18.2-26.0$ & 2002Ish \\
\hline${ }^{17} \mathrm{O}$ & $3 a$ & 23.46 & 11 & 5.95 & 37 & 0.783 & 29 & & & & & & & 0.783 & 29 & $18.5-26.5$ & 2002Ish \\
\hline & $3 \mathrm{~b}$ & 23.42 & 15 & 5.87 & 55 & 0.774 & 42 & & & & & & & 0.774 & 42 & $18.5-26.5$ & 2002Ish \\
\hline${ }^{18} \mathrm{O}$ & $3 a$ & 19.12 & 13 & 2.31 & 61 & 0.075 & 20 & 24.19 & 18 & 5.24 & 87 & 0.413 & 54 & 0.488 & 58 & $18.5-26.0$ & 2002Ish \\
\hline nat $\mathrm{O}$ & 0 & 23.64 & 4 & 5.88 & 13 & 1.044 & 16 & & & & & & & 1.044 & 16 & $18.9-27.9$ & 1985Ahr \\
\hline & 0 & 23.90 & 3 & 6.23 & 10 & 1.126 & 13 & & & & & & & 1.126 & 13 & $18.6-27.8$ & 1975Ahr \\
\hline${ }^{19} \mathrm{~F}$ & $3 a$ & 24.09 & 90 & 24.69 & 215 & 2.038 & 255 & & & & & & & 2.038 & 255 & $10.0-24.0$ & 2002Ish \\
\hline${ }^{23} \mathrm{Na}$ & 0 & 17.57 & 17 & 3.75 & 45 & 0.233 & 41 & 21.26 & 10 & 4.35 & 54 & 0.505 & 66 & 0.738 & 78 & $14.2-23.0$ & 1981Ish \\
\hline & $1 \mathrm{a}$ & 19.99 & 33 & 8.40 & 82 & 0.618 & 64 & 24.78 & 13 & 2.20 & 76 & 0.090 & 31 & 0.708 & 71 & $14.5-25.9$ & $1965 \mathrm{Wyc}$ \\
\hline & $1 b$ & 20.17 & 40 & 8.92 & 120 & 0.646 & 87 & 24.79 & 13 & 1.99 & 75 & 0.079 & 31 & 0.726 & 92 & $14.5-25.9$ & $1965 \mathrm{Wyc}$ \\
\hline & $3 a$ & 17.61 & 20 & 3.83 & 35 & 0.241 & 42 & 21.11 & 11 & 4.18 & 57 & 0.440 & 62 & 0.681 & 75 & $14.3-23.0$ & 2002Ish \\
\hline & $3 \mathrm{~b}$ & 17.51 & 29 & 3.58 & 77 & 0.219 & 59 & 21.08 & 13 & 4.33 & 63 & 0.466 & 77 & 0.685 & 97 & $14.3-23.0$ & 2002Ish \\
\hline & 4 & 17.91 & 12 & 5.22 & 26 & 0.170 & 16 & 25.55 & 38 & 12.86 & 122 & 0.476 & 56 & 0.646 & 58 & $12.6-25.7$ & 1971Alv \\
\hline${ }^{24} \mathrm{Mg}$ & 0 & 19.46 & 14 & 2.80 & 46 & 0.275 & 72 & 24.30 & 43 & 9.80 & 214 & 1.034 & 240 & 1.309 & 251 & $16.5-27.0$ & 1966Dol \\
\hline & $3 a$ & 19.87 & 5 & 3.09 & 12 & 0.415 & 16 & 24.59 & 8 & 4.31 & 34 & 0.353 & 24 & 0.768 & 29 & $16.3-27.0$ & 2003Var \\
\hline & $3 \mathrm{~b}$ & 19.83 & 6 & 3.19 & 19 & 0.421 & 25 & 24.67 & 8 & 4.62 & 47 & 0.373 & 37 & 0.794 & 44 & $16.3-27.0$ & 2003Var \\
\hline & $3 a$ & 20.02 & 7 & 3.48 & 18 & 0.456 & 20 & 24.89 & 8 & 3.91 & 43 & 0.351 & 31 & 0.806 & 37 & $15.2-27.0$ & 2002Ish \\
\hline & $3 \mathrm{~b}$ & 19.90 & 8 & 3.12 & 20 & 0.424 & 25 & 24.85 & 9 & 4.33 & 54 & 0.395 & 42 & 0.819 & 49 & $15.2-27.0$ & 2002Ish \\
\hline${ }^{25} \mathrm{Mg}$ & $3 a$ & 22.73 & 17 & 8.44 & 26 & 1.170 & 40 & & & & & & & 1.170 & 40 & $9.0-24.2$ & 2002Ish \\
\hline & $3 \mathrm{~b}$ & 22.41 & 23 & 7.90 & 45 & 1.092 & 59 & & & & & & & 1.092 & 59 & $9.0-24.2$ & 2002Ish \\
\hline & 4 & 24.56 & 44 & 13.58 & 87 & 1.327 & 122 & & & & & & & 1.327 & 122 & $14.1-23.9$ & 1971Alv \\
\hline${ }^{26} \mathrm{Mg}$ & $3 a$ & 17.39 & 5 & 2.36 & 18 & 0.171 & 12 & 23.83 & 9 & 7.57 & 35 & 1.177 & 46 & 1.348 & 47 & $16.1-26.5$ & 2003Var \\
\hline & $3 \mathrm{~b}$ & 17.36 & 11 & 1.88 & 30 & 0.137 & 18 & 23.81 & 10 & 8.15 & 43 & 1.263 & 56 & 1.401 & 59 & $16.1-26.5$ & 2003Var \\
\hline & $3 a$ & 17.47 & 10 & 2.08 & 35 & 0.137 & 21 & 23.83 & 21 & 7.30 & 72 & 1.059 & 90 & 1.196 & 92 & $16.4-26.0$ & 2002Ish \\
\hline & $3 \mathrm{~b}$ & 17.47 & 22 & 1.57 & 51 & 0.105 & 29 & 23.83 & 24 & 7.97 & 89 & 1.149 & 111 & 1.254 & 115 & $16.4-26.0$ & 2002Ish \\
\hline & 4 & 17.45 & 3 & 2.67 & 11 & 0.179 & 7 & 22.91 & 13 & 5.02 & 30 & 0.412 & 29 & 0.591 & 29 & $16.0-25.7$ & 1971Fu2 \\
\hline${ }^{\text {nat }} \mathrm{Mg}$ & $1 \mathrm{a}$ & 22.32 & 25 & 8.72 & 47 & 0.802 & 36 & & & & & & & 0.802 & 36 & $15.1-26.6$ & 1965 Wyс \\
\hline & $1 b$ & 20.18 & 18 & 4.56 & 43 & 0.548 & 39 & & & & & & & 0.548 & 39 & $15.1-26.6$ & $1965 \mathrm{Wyc}$ \\
\hline${ }^{27} \mathrm{Al}$ & 0 & 21.00 & 10 & 8.62 & 36 & 1.260 & 44 & & & & & & & 1.260 & 44 & $14.0-24.1$ & $1985 \mathrm{Ahr}$ \\
\hline
\end{tabular}


Table 2 (continued)

\begin{tabular}{|c|c|c|c|c|c|c|c|c|c|c|c|c|c|c|c|c|c|}
\hline \multirow{2}{*}{$\begin{array}{l}\text { Nucl } \\
\end{array}$} & \multirow{2}{*}{$\frac{\text { Id }}{0}$} & \multicolumn{2}{|c|}{$\begin{array}{c}E_{r, 1} \\
(\mathrm{MeV})\end{array}$} & \multicolumn{2}{|c|}{$\begin{array}{c}\Gamma_{r, 1} \\
(\mathrm{MeV})\end{array}$} & \multicolumn{2}{|l|}{$s_{1}$} & \multicolumn{2}{|c|}{$\begin{array}{c}E_{r, 2} \\
(\mathrm{MeV})\end{array}$} & $\begin{array}{c}\Gamma_{r, 2} \\
(\mathrm{MeV}\end{array}$ & & $s_{2}$ & & $s$ & & $\begin{array}{c}\varepsilon_{\min }-\varepsilon_{\max } \\
(\mathrm{MeV})\end{array}$ & Ref \\
\hline & & 20.98 & 8 & 8.77 & 27 & 1.272 & 33 & & & & & & & 1.272 & 33 & $14.2-24.4$ & 1975Ahr \\
\hline & 0 & 21.08 & 8 & 8.19 & 29 & 1.189 & 34 & & & & & & & 1.189 & 34 & $14.2-24.4$ & $1972 \mathrm{Ahr}$ \\
\hline & 0 & 21.11 & 7 & 8.60 & 26 & 1.247 & 32 & & & & & & & 1.247 & 32 & $14.2-24.4$ & $1975 \mathrm{Ahr}$ \\
\hline & $3 a$ & 20.62 & 8 & 5.32 & 14 & 0.876 & 21 & & & & & & & 0.876 & 21 & $14.2-23.0$ & 2002Ish \\
\hline & 4 & 21.01 & 3 & 7.15 & 5 & 0.377 & 3 & & & & & & & 0.377 & 3 & $14.0-24.4$ & 1966Fu1 \\
\hline${ }^{28} \mathrm{Si}$ & $3 a$ & 19.88 & 16 & 2.91 & 22 & 0.418 & 104 & 21.84 & 20 & 3.39 & 54 & 0.464 & 128 & 0.882 & 165 & $16.7-23.0$ & 2003Var \\
\hline & $3 \mathrm{~b}$ & 19.78 & 36 & 2.68 & 97 & 0.305 & 280 & 21.68 & 45 & 4.12 & 113 & 0.630 & 358 & 0.935 & 454 & $16.7-23.0$ & 2003Var \\
\hline & $3 a$ & 18.13 & 3 & 0.55 & 9 & 0.059 & 8 & 20.61 & 6 & 3.38 & 13 & 0.655 & 20 & 0.714 & 22 & $16.8-23.0$ & 2002Ish \\
\hline & $3 \mathrm{~b}$ & 18.85 & 30 & 1.73 & 49 & 0.134 & 73 & 20.85 & 20 & 3.65 & 33 & 0.595 & 91 & 0.729 & 117 & $16.8-23.0$ & 2002Ish \\
\hline${ }^{29} \mathrm{Si}$ & $3 a$ & 20.97 & 11 & 6.88 & 25 & 0.941 & 29 & & & & & & & 0.941 & 29 & $14.2-23.0$ & 2002Ish \\
\hline & $3 \mathrm{~b}$ & 20.91 & 16 & 6.69 & 46 & 0.923 & 47 & & & & & & & 0.923 & 47 & $14.2-23.0$ & 2002Ish \\
\hline${ }^{30} \mathrm{Si}$ & $3 a$ & 21.32 & 21 & 9.16 & 48 & 0.916 & 48 & & & & & & & 0.916 & 48 & $14.2-23.0$ & 2002Ish \\
\hline & $3 \mathrm{~b}$ & 21.25 & 29 & 8.88 & 82 & 0.899 & 75 & & & & & & & 0.899 & 75 & $14.2-23.0$ & 2002Ish \\
\hline${ }^{\text {nat }} \mathrm{Si}$ & 0 & 20.45 & 3 & 4.85 & 10 & 0.929 & 17 & 25.24 & 19 & 2.10 & 59 & 0.063 & 17 & 0.992 & 24 & $16.4-25.8$ & $1975 \mathrm{Ahr}$ \\
\hline${ }^{32} \mathrm{~S}$ & 0 & 19.57 & 9 & 5.10 & 35 & 0.583 & 28 & & & & & & & 0.583 & 28 & $14.7-23.0$ & 1968Dol \\
\hline & $3 a$ & 21.21 & 12 & 6.08 & 16 & 1.098 & 36 & & & & & & & 1.098 & 36 & $14.4-23.0$ & 2002Ish \\
\hline & $3 \mathrm{~b}$ & 21.77 & 20 & 7.04 & 41 & 1.288 & 78 & & & & & & & 1.288 & 78 & $14.4-23.0$ & 2002Ish \\
\hline${ }^{34} \mathrm{~S}$ & 0 & 21.66 & 76 & 13.43 & 148 & 1.963 & 299 & & & & & & & 1.963 & 299 & $12.0-25.0$ & 1986Ass \\
\hline & $3 a$ & 22.32 & 22 & 17.94 & 75 & 2.333 & 98 & & & & & & & 2.333 & 98 & $14.1-25.1$ & 2003Var \\
\hline & $3 \mathrm{~b}$ & 22.78 & 41 & 16.62 & 136 & 2.318 & 198 & & & & & & & 2.318 & 198 & $12.3-25.1$ & 2003Var \\
\hline & $3 a$ & 21.23 & 16 & 8.94 & 29 & 1.337 & 43 & & & & & & & 1.337 & 43 & $11.0-25.0$ & 2002Ish \\
\hline & $3 \mathrm{~b}$ & 21.95 & 36 & 10.44 & 107 & 1.551 & 144 & & & & & & & 1.551 & 144 & $11.0-25.0$ & 2002Ish \\
\hline${ }^{\text {nat }} \mathrm{S}$ & 0 & 20.42 & 11 & 5.74 & 40 & 0.887 & 50 & & & & & & & 0.887 & 50 & $17.2-23.6$ & $1965 \mathrm{Wyc}$ \\
\hline${ }^{40} \mathrm{Ar}$ & $3 a$ & 19.91 & 21 & 11.50 & 51 & 1.648 & 62 & & & & & & & 1.648 & 62 & $10.5-25.0$ & 2002Ish \\
\hline & $3 \mathrm{~b}$ & 20.54 & 59 & 13.98 & 160 & 1.857 & 204 & & & & & & & 1.857 & 204 & $10.5-25.0$ & 2002Ish \\
\hline${ }^{\text {nat }} \mathrm{K}$ & 13 & 21.28 & 3 & 7.40 & 10 & 0.443 & 5 & & & & & & & 0.443 & 5 & $16.0-25.9$ & 1974Ve1 \\
\hline${ }^{40} \mathrm{Ca}$ & 0 & 20.72 & 11 & 6.44 & 51 & 1.755 & 102 & & & & & & & 1.755 & 102 & $17.2-23.7$ & 1966Dol \\
\hline & $3 a$ & 20.14 & 5 & 5.05 & 20 & 1.201 & 28 & & & & & & & 1.201 & 28 & $17.2-24.0$ & 2003Ero \\
\hline & $3 \mathrm{~b}$ & 20.11 & 10 & 5.09 & 34 & 1.202 & 56 & & & & & & & 1.202 & 56 & $17.2-24.0$ & 2003Ero \\
\hline${ }^{42} \mathrm{Ca}$ & $3 a$ & 20.53 & 16 & 9.75 & 58 & 1.678 & 83 & & & & & & & 1.678 & 83 & $15.2-23.0$ & 2003Ero \\
\hline & $3 \mathrm{~b}$ & 20.59 & 24 & 8.95 & 95 & 1.622 & 151 & & & & & & & 1.622 & 151 & $15.2-23.0$ & 2003Ero \\
\hline${ }^{44} \mathrm{Ca}$ & $3 a$ & 20.08 & 19 & 12.45 & 102 & 1.858 & 110 & & & & & & & 1.858 & 110 & $15.5-26.0$ & 2003Ero \\
\hline & $3 \mathrm{~b}$ & 20.38 & 47 & 12.76 & 154 & 1.892 & 207 & & & & & & & 1.892 & 207 & $12.5-26.0$ & 2003Ero \\
\hline${ }^{48} \mathrm{Ca}$ & 0 & 19.90 & 18 & 6.42 & 96 & 1.512 & 199 & & & & & & & 1.512 & 199 & $17.9-21.6$ & 1987OKe \\
\hline & $3 a$ & 19.95 & 18 & 7.82 & 67 & 1.461 & 90 & & & & & & & 1.461 & 90 & $15.5-23.0$ & 2003Ero \\
\hline & $3 \mathrm{~b}$ & 19.80 & 28 & 7.11 & 100 & 1.375 & 160 & & & & & & & 1.375 & 160 & $15.5-23.0$ & 2003Ero \\
\hline${ }^{\text {nat }} \mathrm{Ca}$ & 0 & 20.09 & 2 & 5.07 & 8 & 1.241 & 13 & & & & & & & 1.241 & 13 & $15.1-24.0$ & $1975 \mathrm{Ahr}$ \\
\hline & 0 & 19.98 & 3 & 5.07 & 9 & 1.198 & 15 & & & & & & & 1.198 & 15 & $15.2-24.0$ & 1972Ahr \\
\hline & 0 & 19.62 & 3 & 4.65 & 9 & 1.045 & 14 & & & & & & & 1.045 & 14 & $15.0-24.0$ & $1968 \mathrm{Bez}$ \\
\hline & $1 \mathrm{a}$ & 20.42 & 7 & 3.44 & 16 & 0.713 & 18 & & & & & & & 0.713 & 18 & $15.4-24.0$ & $1965 \mathrm{Wyc}$ \\
\hline & $1 \mathrm{~b}$ & 20.25 & 12 & 3.36 & 24 & 0.701 & 34 & & & & & & & 0.701 & 34 & $15.4-24.0$ & $1965 \mathrm{Wyc}$ \\
\hline & 2 & 20.02 & 4 & 5.04 & 13 & 1.171 & 22 & & & & & & & 1.171 & 22 & $9.4-24.0$ & 1985Ahr \\
\hline${ }^{46} \mathrm{Ti}$ & $3 a$ & 19.95 & 9 & 7.99 & 24 & 1.393 & 30 & & & & & & & 1.393 & 30 & $13.2-25.0$ & 2002Ish \\
\hline & $3 \mathrm{~b}$ & 19.98 & 25 & 8.77 & 70 & 1.451 & 103 & & & & & & & 1.451 & 103 & $13.2-25.0$ & 2002Ish \\
\hline${ }^{48} \mathrm{Ti}$ & $3 a$ & 20.13 & 25 & 9.98 & 75 & 1.352 & 88 & & & & & & & 1.352 & 88 & $14.5-23.0$ & 2002Ish \\
\hline & $3 \mathrm{~b}$ & 20.43 & 59 & 11.23 & 187 & 1.472 & 246 & & & & & & & 1.472 & 246 & $14.5-23.0$ & 2002Ish \\
\hline${ }^{51} \mathrm{~V}$ & 4 & 18.18 & 6 & 5.29 & 14 & 0.701 & 28 & 21.37 & 15 & 3.36 & 63 & 0.127 & 30 & 0.828 & 41 & $14.1-22.9$ & 1962Fu1 \\
\hline & $3 a$ & 17.67 & 16 & 3.08 & 135 & 0.191 & 150 & 22.71 & 57 & 16.27 & 236 & 2.443 & 452 & 2.634 & 476 & $15.2-25.0$ & 2003Var \\
\hline & $3 \mathrm{~b}$ & 17.96 & 36 & 4.91 & 123 & 0.646 & 288 & 22.87 & 65 & 8.20 & 396 & 1.053 & 638 & 1.699 & 700 & $15.2-25.0$ & 2003Var \\
\hline & $4 a$ & 17.29 & 4 & 2.59 & 6 & 0.304 & 6 & 19.90 & 2 & 1.04 & 4 & 0.138 & 5 & 0.442 & 8 & $11.0-24.5$ & 1969Gor \\
\hline & $4 \mathrm{~b}$ & 15.58 & 122 & 4.69 & 963 & 0.048 & 19 & 18.40 & 23 & 3.67 & 43 & 0.549 & 44 & 0.597 & 48 & $11.0-24.5$ & 1969 Gor \\
\hline${ }^{52} \mathrm{Cr}$ & $3 a$ & 19.16 & 8 & 6.70 & 24 & 1.075 & 27 & & & & & & & 1.075 & 27 & $14.3-23.0$ & 2002Ish \\
\hline & $3 \mathrm{~b}$ & 19.36 & 22 & 7.52 & 63 & 1.146 & 84 & & & & & & & 1.146 & 84 & $14.3-23.0$ & 2002Ish \\
\hline & $4 \mathrm{a}$ & 18.21 & 4 & 5.09 & 9 & 0.782 & 12 & & & & & & & 0.782 & 12 & $14.1-23.0$ & 1969Gor \\
\hline & $4 \mathrm{~b}$ & 18.65 & 6 & 6.36 & 16 & 0.977 & 23 & & & & & & & 0.977 & 23 & $14.1-23.0$ & 1969Gor \\
\hline${ }^{55} \mathrm{Mn}$ & 4 & 16.43 & 8 & 2.91 & 47 & 0.122 & 37 & 20.13 & 23 & 11.28 & 60 & 1.107 & 79 & 1.229 & 87 & $14.0-23.0$ & 1979Al2 \\
\hline & 4 & 17.02 & 25 & 3.88 & 63 & 0.368 & 172 & 20.05 & 63 & 7.60 & 140 & 0.693 & 245 & 1.061 & 299 & $14.1-22.8$ & 1974Car \\
\hline & $4 \mathrm{a}$ & 16.47 & 10 & 5.16 & 18 & 0.555 & 37 & 19.26 & 8 & 3.75 & 31 & 0.223 & 33 & 0.778 & 49 & $10.1-23.0$ & 1970Is1 \\
\hline & $4 \mathrm{~b}$ & 16.45 & 11 & 5.14 & 18 & 0.547 & 39 & 19.26 & 9 & 3.90 & 40 & 0.235 & 39 & 0.782 & 56 & $10.1-23.0$ & 1970Is1 \\
\hline${ }^{54} \mathrm{Fe}$ & 0 & 19.39 & 8 & 5.69 & 30 & 1.606 & 54 & & & & & & & 1.606 & 54 & $16.0-23.0$ & 1978 Nor \\
\hline${ }^{59} \mathrm{Co}$ & 4 & 16.44 & 9 & 2.42 & 37 & 0.087 & 28 & 18.68 & 22 & 8.63 & 46 & 0.922 & 35 & 1.009 & 45 & $14.0-20.9$ & 1979Al2 \\
\hline & 4 & 15.50 & 16 & 3.53 & 49 & 0.377 & 93 & 18.56 & 21 & 5.32 & 80 & 0.525 & 120 & 0.901 & 151 & $14.1-22.8$ & 1964Baz \\
\hline & $4 \mathrm{a}$ & 16.64 & 9 & 4.68 & 14 & 0.416 & 38 & 19.43 & 10 & 5.96 & 29 & 0.466 & 45 & 0.882 & 59 & $12.1-24.0$ & 1969Gor \\
\hline & $4 \mathrm{~b}$ & 16.63 & 9 & 4.67 & 14 & 0.414 & 38 & 19.43 & 10 & 5.99 & 29 & 0.469 & 44 & 0.883 & 58 & $12.1-24.0$ & 1969Gor \\
\hline${ }^{58} \mathrm{Ni}$ & $3 a$ & 18.87 & 6 & 6.16 & 17 & 0.958 & 19 & & & & & & & 0.958 & 19 & $14.1-22.0$ & 2003Var \\
\hline & $3 \mathrm{~b}$ & 18.78 & 12 & 6.03 & 36 & 0.942 & 47 & & & & & & & 0.942 & 47 & $14.1-22.0$ & 2003Var \\
\hline & $3 a$ & 19.14 & 13 & 7.22 & 38 & 1.078 & 44 & & & & & & & 1.078 & 44 & $14.4-22.0$ & 2002Ish \\
\hline
\end{tabular}


Table 2 (continued)

\begin{tabular}{|c|c|c|c|c|c|c|c|c|c|c|c|c|c|c|c|c|c|}
\hline \multirow{2}{*}{ Nucl } & \multirow{2}{*}{$\frac{\text { Id }}{3 \mathrm{~b}}$} & \multicolumn{2}{|c|}{$\begin{array}{c}E_{r, 1} \\
(\mathrm{MeV})\end{array}$} & \multicolumn{2}{|c|}{$\begin{array}{c}\Gamma_{r, 1} \\
(\mathrm{MeV})\end{array}$} & \multicolumn{2}{|l|}{$s_{1}$} & \multicolumn{2}{|c|}{$\begin{array}{c}E_{r, 2} \\
(\mathrm{MeV})\end{array}$} & $\begin{aligned} & \Gamma_{r, 2} \\
&(\mathrm{MeI}\end{aligned}$ & & $s_{2}$ & & $s$ & & $\begin{array}{c}\varepsilon_{\min }-\varepsilon_{\max } \\
(\mathrm{MeV})\end{array}$ & Ref \\
\hline & & 18.92 & 25 & 6.77 & 75 & 1.022 & 100 & & & & & & & 1.022 & 100 & $14.4-22.0$ & 2002Ish \\
\hline & 4 & 18.53 & 7 & 7.97 & 22 & 0.330 & 9 & & & & & & & 0.330 & 9 & $12.2-21.8$ & 1974Fu3 \\
\hline & 4 & 19.22 & 3 & 9.47 & 8 & 0.460 & 5 & & & & & & & 0.460 & 5 & $14.1-22.0$ & 1970Gor \\
\hline${ }^{60} \mathrm{Ni}$ & $3 a$ & 16.59 & 29 & 3.17 & 94 & 0.218 & 135 & 19.39 & 44 & 7.82 & 49 & 1.069 & 152 & 1.287 & 203 & $12.1-21.0$ & 2003Var \\
\hline & $3 \mathrm{~b}$ & 16.40 & 18 & 2.02 & 105 & 0.103 & 86 & 19.04 & 46 & 7.78 & 68 & 1.202 & 126 & 1.305 & 152 & $12.1-21.0$ & 2003Var \\
\hline & 4 & 16.23 & 7 & 1.83 & 56 & 0.072 & 35 & 18.25 & 18 & 7.09 & 24 & 0.746 & 48 & 0.818 & 59 & $14.0-20.9$ & $1974 \mathrm{Fu} 3$ \\
\hline & 4 & 16.51 & 10 & 2.81 & 29 & 0.143 & 29 & 19.03 & 11 & 6.21 & 13 & 0.606 & 34 & 0.749 & 45 & $11.4-22.0$ & 1970Gor \\
\hline${ }^{63} \mathrm{Cu}$ & $3 a$ & 16.79 & 31 & 5.76 & 68 & 0.844 & 175 & 20.37 & 32 & 4.56 & 243 & 0.274 & 211 & 1.118 & 274 & $14.0-21.0$ & 2003Var \\
\hline & $3 \mathrm{~b}$ & 16.67 & 74 & 5.44 & 197 & 0.766 & 510 & 20.43 & 108 & 5.62 & 776 & 0.377 & 774 & 1.143 & 927 & $14.0-21.0$ & 2003Var \\
\hline & $3 a$ & 16.23 & 32 & 2.62 & 229 & 0.173 & 273 & 20.87 & 244 & 15.61 & 761 & 1.849 & 1007 & 2.023 & 1001 & $14.5-21.0$ & 2002Ish \\
\hline & $3 \mathrm{~b}$ & 16.48 & 76 & 4.38 & 227 & 0.554 & 532 & 20.59 & 141 & 6.26 & 937 & 0.581 & 1147 & 1.135 & 1264 & $14.5-21.0$ & 2002Ish \\
\hline & 4 & 16.51 & 12 & 5.26 & 33 & 0.557 & 49 & 19.72 & 21 & 3.81 & 112 & 0.117 & 49 & 0.674 & 69 & $14.2-20.7$ & 1964Fu1 \\
\hline & 5 & 16.23 & 39 & 2.18 & 237 & 0.083 & 155 & 18.43 & 238 & 13.14 & 859 & 1.052 & 417 & 1.135 & 445 & $14.2-20.7$ & 2016Va1 \\
\hline & 6 & 17.01 & 10 & 4.80 & 15 & 0.575 & 37 & 19.16 & 11 & 2.55 & 49 & 0.078 & 28 & 0.653 & 46 & $14.0-21.0$ & 1968Su1 \\
\hline${ }^{65} \mathrm{Cu}$ & $3 a$ & 17.23 & 8 & 8.38 & 43 & 1.176 & 45 & & & & & & & 1.176 & 45 & $14.2-21.0$ & 2003Var \\
\hline & $3 \mathrm{~b}$ & 17.16 & 29 & 7.87 & 138 & 1.126 & 183 & & & & & & & 1.126 & 183 & $14.2-21.0$ & 2003Var \\
\hline & $3 a$ & 17.72 & 32 & 10.96 & 184 & 1.628 & 229 & & & & & & & 1.628 & 229 & $14.5-21.0$ & 2002Ish \\
\hline & $3 \mathrm{~b}$ & 17.35 & 46 & 8.80 & $239^{\circ}$ & 1.379 & 335 & & & & & & & 1.379 & 335 & $14.5-21.0$ & 2002Ish \\
\hline & 4 & 16.92 & 6 & 7.04 & 31 & 0.847 & 31 & & & & & & & 0.847 & 31 & $14.2-19.9$ & 1964Fu1 \\
\hline & 5 & 17.40 & 17 & 9.12 & 95 & 1.072 & 85 & & & & & & & 1.072 & 85 & $14.2-20.9$ & 2016Va1 \\
\hline${ }^{\text {nat }} \mathrm{Cu}$ & $1 \mathrm{a}$ & 17.91 & 9 & 5.24 & 19 & 0.873 & 21 & & & & & & & 0.873 & 21 & $14.4-24.9$ & $1965 \mathrm{Wyc}$ \\
\hline & $1 b$ & 17.87 & 25 & 6.09 & 68 & 0.944 & 94 & & & & & & & 0.944 & 94 & $14.4-24.9$ & $1965 \mathrm{Wyc}$ \\
\hline & 4 & 17.43 & 4 & 8.06 & 17 & 0.901 & 13 & & & & & & & 0.901 & 13 & $14.2-24.7$ & $1964 \mathrm{Ful}$ \\
\hline & $4 a$ & 17.59 & 7 & 9.96 & 34 & 1.462 & 40 & & & & & & & 1.462 & 40 & $14.1-25.0$ & 1970Is2 \\
\hline & $4 \mathrm{~b}$ & 17.40 & 10 & 8.84 & 46 & 1.326 & 60 & & & & & & & 1.326 & 60 & $14.1-25.0$ & 1970Is2 \\
\hline & $4 \mathrm{a}$ & 16.46 & 53 & 12.98 & 212 & 1.587 & 214 & & & & & & & 1.587 & 214 & $14.6-23.2$ & 1968Tom \\
\hline & $4 \mathrm{~b}$ & 16.39 & 79 & 14.61 & 485 & 1.776 & 533 & & & & & & & 1.776 & 533 & $14.6-23.2$ & 1968Tom \\
\hline${ }^{64} \mathrm{Zn}$ & 8 & 16.37 & 19 & 3.84 & 46 & 0.303 & 86 & 19.49 & 19 & 5.82 & 120 & 0.457 & 139 & 0.760 & 163 & $14.0-20.8$ & 1976Ca1 \\
\hline${ }^{65} \mathrm{Zn}$ & $1 \mathrm{a}$ & 16.06 & 12 & 2.26 & 42 & 0.077 & 23 & 18.87 & 19 & 8.62 & 31 & 0.842 & 26 & 0.919 & 35 & $12.0-21.0$ & 2003Rod \\
\hline & $1 \mathrm{~b}$ & 16.66 & 28 & 4.86 & 53 & 0.416 & 96 & 19.70 & 19 & 5.03 & 115 & 0.306 & 117 & 0.722 & 151 & $12.0-21.0$ & 2003Rod \\
\hline${ }^{70} \mathrm{Ge}$ & 0 & 15.31 & 23 & 7.19 & 60 & 1.642 & 117 & & & & & & & 1.642 & 117 & $10.0-20.0$ & $1975 \mathrm{Mcc}$ \\
\hline & 8 & 17.03 & 10 & 8.19 & 41 & 1.076 & 46 & & & & & & & 1.076 & 46 & $13.1-20.8$ & 1976Ca1 \\
\hline${ }^{72} \mathrm{Ge}$ & 0 & 17.85 & 16 & 6.22 & 54 & 1.461 & 84 & & & & & & & 1.461 & 84 & $10.0-24.0$ & $1975 \mathrm{Mcc}$ \\
\hline & 8 & 16.90 & 7 & 8.03 & 30 & 1.244 & 38 & & & & & & & 1.244 & 38 & $13.1-20.8$ & 1976Ca1 \\
\hline${ }^{74} \mathrm{Ge}$ & 8 & 14.42 & 16 & 2.46 & 83 & 0.115 & 84 & 17.47 & 29 & 8.37 & 85 & 1.159 & 166 & 1.274 & 186 & $13.1-20.8$ & 1976Ca1 \\
\hline & 0 & 13.97 & 25 & 0.051 & 16278 & 0.001 & 28 & 16.93 & 36 & 9.31 & 100 & 1.792 & 179 & 1.793 & 181 & $12.0-20.0$ & $1975 \mathrm{Mcc}$ \\
\hline${ }^{76} \mathrm{Ge}$ & 8 & 15.42 & 72 & 3.62 & 383 & 0.197 & 504 & 18.69 & 297 & 13.81 & 541 & 1.604 & 354 & 1.801 & 616 & $13.1-20.8$ & $1976 \mathrm{Ca} 1$ \\
\hline & 0 & 16.91 & 25 & 9.10 & 64 & 1.499 & 155 & 29.20 & 899 & 14.88 & 1212 & 1.033 & 1960 & 2.532 & 1966 & $10.0-24.0$ & $1975 \mathrm{Mcc}$ \\
\hline${ }^{75} \mathrm{As}$ & 4 & 15.25 & 24 & 4.73 & 52 & 0.401 & 118 & 18.16 & 25 & 6.73 & 71 & 0.563 & 142 & 0.964 & 185 & $13.1-20.9$ & 1969Be1 \\
\hline & 8 & 15.30 & 77 & 4.91 & 173 & 0.494 & 543 & 18.40 & 115 & 8.18 & 281 & 0.791 & 706 & 1.285 & 891 & $13.1-20.8$ & 1976Ca1 \\
\hline${ }^{76} \mathrm{Se}$ & 0 & 15.86 & 7 & 6.50 & 35 & 1.365 & 56 & & & & & & & 1.365 & 56 & $13.1-19.7$ & 1978Gur \\
\hline & 8 & 17.21 & 12 & 10.66 & 55 & 1.555 & 72 & & & & & & & 1.555 & 72 & $13.1-20.8$ & $1976 \mathrm{Ca} 1$ \\
\hline${ }^{78} \mathrm{Se}$ & 8 & 15.23 & 23 & 4.67 & 64 & 0.527 & 123 & 18.76 & 19 & 5.68 & 107 & 0.512 & 150 & 1.039 & 194 & $13.1-20.8$ & 1976Ca1 \\
\hline${ }^{80} \mathrm{Se}$ & 5 & 16.84 & 8 & 7.45 & 30 & 1.349 & 46 & & & & & & & 1.349 & 46 & $13.1-20.0$ & 2016Va1 \\
\hline & 8 & 16.48 & 17 & 6.43 & 48 & 1.152 & 94 & & & & & & & 1.152 & 94 & $13.1-17.0$ & 1976Ca1 \\
\hline${ }^{82} \mathrm{Se}$ & 0 & 16.13 & 5 & 5.81 & 23 & 1.339 & 39 & & & & & & & 1.339 & 39 & $13.1-19.9$ & 1978Gur \\
\hline & 8 & 16.75 & 5 & 6.08 & 20 & 1.168 & 29 & & & & & & & 1.168 & 29 & $13.1-20.8$ & 1976Ca1 \\
\hline${ }^{n a t} R b$ & 4 & 16.92 & 2 & 4.93 & 6 & 1.147 & 12 & & & & & & & 1.147 & 12 & $10.6-17.9$ & 1971Lep \\
\hline${ }^{\text {nat }} \mathrm{Sr}$ & 4 & 16.99 & 2 & 5.03 & 5 & 1.227 & 12 & & & & & & & 1.227 & 12 & $10.9-17.9$ & 1971Lep \\
\hline${ }^{89} \mathrm{Y}$ & 4 & 16.82 & 1 & 4.42 & 4 & 1.175 & 8 & & & & & & & 1.175 & 8 & $14.0-19.0$ & 1971Le1 \\
\hline & $3 a$ & 16.89 & 5 & 4.50 & 29 & 1.255 & 57 & & & & & & & 1.255 & 57 & $15.3-19.0$ & 2003Var \\
\hline & $3 \mathrm{~b}$ & 16.91 & 22 & 4.57 & 106 & 1.268 & 268 & & & & & & & 1.268 & 268 & $15.3-19.0$ & 2003Var \\
\hline & 4 & 17.65 & 1 & 4.89 & 5 & 0.396 & 3 & & & & & & & 0.396 & 3 & $15.3-19.0$ & 1999Bel \\
\hline & 4 & 16.84 & 1 & 4.14 & 3 & 0.896 & 4 & & & & & & & 0.896 & 4 & $14.0-18.9$ & 1967Be2 \\
\hline & 12 & 16.93 & 3 & 4.01 & 9 & 0.956 & 20 & & & & & & & 0.956 & 20 & $14.0-18.1$ & $1972 \mathrm{You}$ \\
\hline${ }^{90} \mathrm{Zr}$ & $3 a$ & 16.90 & 5 & 4.13 & 25 & 1.226 & 53 & & & & & & & 1.226 & 53 & $14.9-18.5$ & 2003Var \\
\hline & $3 \mathrm{~b}$ & 16.87 & 16 & 3.97 & 67 & 1.193 & 179 & & & & & & & 1.193 & 179 & $14.9-18.5$ & 2003Var \\
\hline & 4 & 16.93 & 2 & 4.30 & 9 & 0.993 & 17 & & & & & & & 0.993 & 17 & $15.0-18.5$ & 1972Ask \\
\hline & 4 & 16.91 & 1 & 4.18 & 3 & 0.893 & 6 & & & & & & & 0.893 & 6 & $14.0-18.9$ & 1967Be2 \\
\hline & 8 & 16.81 & 1 & 4.31 & 4 & 1.057 & 7 & & & & & & & 1.057 & 7 & $14.0-19.0$ & 1971Le1 \\
\hline${ }^{91} \mathrm{Zr}$ & 4 & 16.64 & 2 & 4.32 & 7 & 0.917 & 11 & & & & & & & 0.917 & 11 & $14.0-18.9$ & 1967Be2 \\
\hline & 5 & 16.63 & 9 & 4.29 & 33 & 0.911 & 47 & & & & & & & 0.911 & 47 & $14.0-18.9$ & 2015Var \\
\hline${ }^{92} \mathrm{Zr}$ & 4 & 16.34 & 2 & 4.70 & 10 & 0.898 & 13 & & & & & & & 0.898 & 13 & $14.0-18.9$ & 1967Be2 \\
\hline${ }^{94} \mathrm{Zr}$ & 4 & 16.35 & 3 & 5.52 & 12 & 0.991 & 17 & & & & & & & 0.991 & 17 & $14.0-18.9$ & 1967Be2 \\
\hline & 5 & 16.47 & 11 & 5.38 & 53 & 1.020 & 73 & & & & & & & 1.020 & 73 & $14.0-19.0$ & 2015Var \\
\hline${ }^{93} \mathrm{Nb}$ & 8 & 16.70 & 2 & 5.18 & 6 & 1.175 & 12 & & & & & & & 1.175 & 12 & $14.0-19.0$ & 1971Le1 \\
\hline${ }^{92} \mathrm{Mo}$ & $3 a$ & 17.28 & 7 & 5.05 & 25 & 1.368 & 52 & & & & & & & 1.368 & 52 & $14.4-19.0$ & 2003Var \\
\hline
\end{tabular}


Table 2 (continued)

\begin{tabular}{|c|c|c|c|c|c|c|c|c|c|c|c|c|c|c|c|c|c|}
\hline \multirow[t]{5}{*}{ Nucl } & Id & \multicolumn{2}{|c|}{$\begin{array}{c}E_{r, 1} \\
(\mathrm{MeV})\end{array}$} & \multicolumn{2}{|c|}{$\begin{array}{c}\Gamma_{r, 1} \\
(\mathrm{MeV})\end{array}$} & \multicolumn{2}{|l|}{$s_{1}$} & \multicolumn{2}{|c|}{$\begin{array}{c}E_{r, 2} \\
(\mathrm{MeV})\end{array}$} & \multicolumn{2}{|c|}{$\begin{array}{c}\Gamma_{r, 2} \\
(\mathrm{MeV})\end{array}$} & $s_{2}$ & & $s$ & & $\begin{array}{c}\varepsilon_{\min }-\varepsilon_{\max } \\
(\mathrm{MeV})\end{array}$ & Ref \\
\hline & $3 \mathrm{~b}$ & 17.21 & 17 & 4.62 & 62 & 1.293 & 160 & & & & & & & 1.293 & 160 & $14.4-19.0$ & 2003Var \\
\hline & 4 & 16.89 & 1 & 4.28 & 4 & 0.781 & 6 & & & & & & & 0.781 & 6 & $14.0-18.9$ & 1974Be3 \\
\hline & $4 a$ & 16.67 & 4 & 5.13 & 22 & 0.961 & 30 & & & & & & & 0.961 & 30 & $14.5-19.0$ & 1970Is3 \\
\hline & $4 \mathrm{~b}$ & 16.64 & 5 & 5.06 & 28 & 0.964 & 39 & & & & & & & 0.964 & 39 & $14.5-19.0$ & 1970Is3 \\
\hline${ }^{94} \mathrm{Mo}$ & 4 & 16.73 & 2 & 6.04 & 6 & 1.268 & 12 & & & & & & & 1.268 & 12 & $9.6-18.9$ & 1974Be3 \\
\hline${ }^{96} \mathrm{Mo}$ & 4 & 16.42 & 7 & 6.52 & 20 & 1.315 & 43 & & & & & & & 1.315 & 43 & $13.2-17.0$ & 1974Be3 \\
\hline${ }^{98} \mathrm{Mo}$ & 4 & 15.96 & 4 & 6.17 & 18 & 1.257 & 28 & & & & & & & 1.257 & 28 & $13.2-18.9$ & 1974Be3 \\
\hline & $4 \mathrm{a}$ & 15.65 & 5 & 5.45 & 23 & 1.317 & 37 & & & & & & & 1.317 & 37 & $13.1-19.0$ & 1970Is3 \\
\hline & $4 \mathrm{~b}$ & 15.67 & 9 & 5.65 & 42 & 1.385 & 82 & & & & & & & 1.385 & 82 & $13.1-19.0$ & 1970Is3 \\
\hline${ }^{100} \mathrm{Mo}$ & 9 & 16.02 & 4 & 8.44 & 18 & 1.515 & 26 & & & & & & & 1.515 & 26 & $12.1-20.0$ & $1974 \mathrm{Be} 3$ \\
\hline${ }^{103} \mathrm{Rh}$ & $3 a$ & 16.59 & 11 & 8.44 & 51 & 1.638 & 82 & & & & & & & 1.638 & 82 & $13.1-19.0$ & 2003Var \\
\hline & $3 \mathrm{~b}$ & 16.62 & 25 & 8.56 & 107 & 1.658 & 193 & & & & & & & 1.658 & 193 & $13.1-19.0$ & 2003Var \\
\hline & 4 & 16.47 & 5 & 8.02 & 21 & 1.543 & 35 & & & & & & & 1.543 & 35 & $13.2-18.9$ & 1974Le1 \\
\hline & 4 & 16.83 & 27 & 7.60 & 56 & 1.913 & 148 & & & & & & & 1.913 & 148 & $10.0-19.0$ & 1962Bog \\
\hline${ }^{108} \mathrm{Pd}$ & 0 & 16.15 & 35 & 8.26 & 48 & 1.390 & 160 & 18.09 & 31 & 1.65 & 137 & 0.075 & 73 & 1.465 & 176 & $10.0-19.0$ & 1969Dea \\
\hline${ }^{\text {nat }} \mathrm{Pd}$ & 4 & 16.24 & 5 & 7.63 & 14 & 1.502 & 26 & & & & & & & 1.502 & 26 & $10.2-17.8$ & 1971Lep \\
\hline${ }^{107} \mathrm{Ag}$ & 4 & 16.05 & 5 & 7.51 & 17 & 1.338 & 26 & & & & & & & 1.338 & 26 & $9.5-19.0$ & 1969Ish \\
\hline & 4 & 16.14 & 5 & 7.18 & 22 & 1.053 & 29 & & & & & & & 1.053 & 29 & $13.1-18.7$ & 1969Be1 \\
\hline & $4 \mathrm{a}$ & 15.78 & 8 & 6.40 & 24 & 1.528 & 44 & & & & & & & 1.528 & 44 & $10.0-19.0$ & 1962Bog \\
\hline & $4 \mathrm{~b}$ & 15.87 & 13 & 6.64 & 31 & 1.581 & 74 & & & & & & & 1.581 & 74 & $10.0-19.0$ & 1962Bog \\
\hline${ }^{109} \mathrm{Ag}$ & 4 & 13.74 & 22 & 3.81 & 154 & 0.337 & 167 & 16.76 & 12 & 4.17 & 53 & 0.423 & 125 & 0.760 & 209 & $13.1-19.0$ & 1969Ish \\
\hline${ }^{\text {nat }} \mathrm{Ag}$ & 4 & 16.39 & 2 & 8.03 & 10 & 1.555 & 16 & & & & & & & 1.555 & 16 & $13.2-18.9$ & 1971Lep \\
\hline${ }^{n a t} \mathrm{Cd}$ & 4 & 16.03 & 2 & 6.69 & 6 & 1.400 & 10 & & & & & & & 1.400 & 10 & $10.2-17.8$ & 1971Lep \\
\hline${ }^{115} \mathrm{In}$ & 4 & 15.91 & 2 & 6.00 & 7 & 1.353 & 13 & & & & & & & 1.353 & 13 & $13.2-17.8$ & 1974Le1 \\
\hline & 4 & 15.79 & 1 & 5.58 & 6 & 1.357 & 12 & & & & & & & 1.357 & 12 & $13.1-17.8$ & 1969Fu1 \\
\hline & 4 & 15.54 & 17 & 5.66 & 38 & 1.584 & 100 & & & & & & & 1.584 & 100 & $10.0-18.0$ & 1962Bog \\
\hline & 5 & 15.63 & 7 & 5.00 & 31 & 1.221 & 53 & & & & & & & 1.221 & 53 & $13.1-17.8$ & 2013Var \\
\hline${ }^{112} \mathrm{Sn}$ & 4 & 15.82 & 6 & 5.87 & 18 & 1.411 & 37 & & & & & & & 1.411 & 37 & $10.9-18.0$ & 1974Sor \\
\hline & 5 & 15.81 & 7 & 5.74 & 27 & 1.388 & 54 & & & & & & & 1.388 & 54 & $13.1-18.0$ & 2009Var \\
\hline${ }^{114} \mathrm{Sn}$ & 4 & 16.08 & 6 & 6.74 & 24 & 1.500 & 48 & & & & & & & 1.500 & 48 & $13.1-18.0$ & 1975Sor \\
\hline & 5 & 16.01 & 6 & 6.50 & 22 & 1.448 & 44 & & & & & & & 1.448 & 44 & $13.1-18.0$ & 2009Var \\
\hline${ }^{116} \mathrm{Sn}$ & 4 & 15.69 & 1 & 5.29 & 6 & 1.302 & 12 & & & & & & & 1.302 & 12 & $13.1-17.9$ & 1974Le1 \\
\hline & 4 & 15.74 & 2 & 4.34 & 8 & 1.049 & 13 & & & & & & & 1.049 & 13 & $13.0-18.0$ & 1969Fu1 \\
\hline & $4 a$ & 15.94 & 4 & 6.14 & 16 & 1.403 & 32 & & & & & & & 1.403 & 32 & $13.1-18.0$ & 1975Sor \\
\hline & $4 \mathrm{~b}$ & 15.96 & 4 & 6.20 & 17 & 1.417 & 35 & & & & & & & 1.417 & 35 & $13.1-18.0$ & 1975Sor \\
\hline & 5 & 15.69 & 1 & 4.94 & 4 & 1.203 & 7 & & & & & & & 1.203 & 7 & $13.1-18.0$ & 2009Var \\
\hline $117 \mathrm{Sn}$ & 4 & 15.77 & 2 & 5.29 & 11 & 1.232 & 20 & & & & & & & 1.232 & 20 & $13.2-17.8$ & 1974Le1 \\
\hline & 4 & 15.77 & 2 & 5.31 & 7 & 1.208 & 11 & & & & & & & 1.208 & 11 & $13.1-17.9$ & 1969Fu1 \\
\hline & $4 a$ & 15.76 & 4 & 5.61 & 15 & 1.298 & 31 & & & & & & & 1.298 & 31 & $13.1-18.0$ & 1975Sor \\
\hline & $4 \mathrm{~b}$ & 15.85 & 6 & 5.88 & 20 & 1.360 & 44 & & & & & & & 1.360 & 44 & $13.1-18.0$ & 1975Sor \\
\hline & 5 & 15.75 & 1 & 5.22 & 3 & 1.209 & 5 & & & & & & & 1.209 & 5 & $13.1-18.0$ & 2009Var \\
\hline${ }^{118} \mathrm{Sn}$ & 4 & 15.55 & 1 & 5.02 & 6 & 1.259 & 12 & & & & & & & 1.259 & 12 & $13.1-17.9$ & 1974Le1 \\
\hline & 4 & 15.70 & 1 & 5.02 & 5 & 1.148 & 8 & & & & & & & 1.148 & 8 & $13.1-17.9$ & 1969Fu1 \\
\hline & $4 \mathrm{a}$ & 15.60 & 5 & 6.42 & 22 & 1.560 & 48 & & & & & & & 1.560 & 48 & $13.1-18.0$ & 1974Sor \\
\hline & $4 \mathrm{~b}$ & 15.65 & 6 & 6.62 & 28 & 1.604 & 63 & & & & & & & 1.604 & 63 & $13.1-18.0$ & 1974Sor \\
\hline & 5 & 15.56 & 1 & 4.69 & 2 & 1.109 & 4 & & & & & & & 1.109 & 4 & $13.1-18.0$ & 2009Var \\
\hline${ }^{119} \mathrm{Sn}$ & 4 & 15.65 & 2 & 5.09 & 7 & 1.144 & 14 & & & & & & & 1.144 & 14 & $13.0-17.9$ & 1969Fu1 \\
\hline & $4 \mathrm{a}$ & 14.85 & 3 & 4.76 & 14 & 0.968 & 22 & & & & & & & 0.968 & 22 & $13.1-17.9$ & 1975Sor \\
\hline & $4 \mathrm{~b}$ & 14.84 & 4 & 4.70 & 17 & 0.956 & 32 & & & & & & & 0.956 & 32 & $13.1-17.9$ & 1975Sor \\
\hline & 5 & 15.72 & 1 & 5.48 & 6 & 1.236 & 9 & & & & & & & 1.236 & 9 & $13.1-18.0$ & 2009Var \\
\hline${ }^{120} \mathrm{Sn}$ & 4 & 15.50 & 1 & 5.26 & 7 & 1.334 & 13 & & & & & & & 1.334 & 13 & $13.1-17.9$ & 1974Le1 \\
\hline & 4 & 15.53 & 1 & 5.03 & 4 & 1.256 & 9 & & & & & & & 1.256 & 9 & $13.1-17.9$ & 1969Fu1 \\
\hline & $4 a$ & 15.49 & 5 & 6.12 & 24 & 1.522 & 51 & & & & & & & 1.522 & 51 & $13.1-18.0$ & 1974Sor \\
\hline & $4 \mathrm{~b}$ & 15.50 & 9 & 6.15 & 34 & 1.528 & 82 & & & & & & & 1.528 & 82 & $13.1-18.0$ & 1974Sor \\
\hline & 5 & 15.45 & 1 & 4.85 & 2 & 1.221 & 4 & & & & & & & 1.221 & 4 & $13.1-18.0$ & 2009Var \\
\hline${ }^{122} \mathrm{Sn}$ & $4 \mathrm{a}$ & 15.45 & 4 & 4.98 & 13 & 1.158 & 26 & & & & & & & 1.158 & 26 & $13.1-18.0$ & 1975Sor \\
\hline & $4 \mathrm{~b}$ & 15.50 & 6 & 5.12 & 18 & 1.194 & 44 & & & & & & & 1.194 & 44 & $13.1-18.0$ & 1975Sor \\
\hline & 5 & 15.35 & 2 & 4.72 & 7 & 1.087 & 11 & & & & & & & 1.087 & 11 & $13.1-18.0$ & 2009Var \\
\hline${ }^{124} \mathrm{Sn}$ & $3 a$ & 15.41 & 5 & 5.06 & 24 & 1.198 & 37 & & & & & & & 1.198 & 37 & $13.1-18.0$ & 2003Var \\
\hline & $3 \mathrm{~b}$ & 15.40 & 9 & 5.00 & 39 & 1.189 & 73 & & & & & & & 1.189 & 73 & $13.1-18.0$ & 2003Var \\
\hline & 4 & 15.39 & 2 & 4.90 & 9 & 1.174 & 16 & & & & & & & 1.174 & 16 & $13.2-17.8$ & 1974Le1 \\
\hline & 4 & 15.30 & 2 & 4.98 & 8 & 1.222 & 16 & & & & & & & 1.222 & 16 & $13.1-17.8$ & 1969Fu1 \\
\hline & $4 \mathrm{a}$ & 15.35 & 4 & 5.81 & 20 & 1.308 & 36 & & & & & & & 1.308 & 36 & $13.1-18.0$ & 1975Sor \\
\hline & $4 \mathrm{~b}$ & 15.39 & 8 & 5.95 & 28 & 1.342 & 62 & & & & & & & 1.342 & 62 & $13.1-18.0$ & 1975Sor \\
\hline & 5 & 15.28 & 1 & 4.90 & 3 & 1.173 & 5 & & & & & & & 1.173 & 5 & $13.1-18.0$ & 2009Var \\
\hline${ }^{\text {nat }} \mathrm{Sb}$ & 4 & 15.62 & 1 & 5.59 & 4 & 1.356 & 8 & & & & & & & 1.356 & 8 & $10.2-17.8$ & 1971Lep \\
\hline${ }^{124} \mathrm{Te}$ & 6 & 15.36 & 2 & 5.81 & 9 & 1.394 & 18 & & & & & & & 1.394 & 18 & $12.0-18.9$ & 1976Le2 \\
\hline
\end{tabular}


Table 2 (continued)

\begin{tabular}{|c|c|c|c|c|c|c|c|c|c|c|c|c|c|c|c|c|c|}
\hline \multirow{2}{*}{$\begin{array}{c}\mathrm{Nucl} \\
{ }^{126} \mathrm{Te}\end{array}$} & \multirow{2}{*}{$\frac{\text { Id }}{6}$} & \multicolumn{2}{|c|}{$\begin{array}{c}E_{r, 1} \\
(\mathrm{MeV})\end{array}$} & \multicolumn{2}{|c|}{$\begin{array}{c}\Gamma_{r, 1} \\
(\mathrm{MeV})\end{array}$} & \multicolumn{2}{|l|}{$s_{1}$} & \multicolumn{2}{|c|}{$\begin{array}{c}E_{r, 2} \\
(\mathrm{MeV})\end{array}$} & $\begin{array}{r}\Gamma_{r} \\
(\mathrm{Me}\end{array}$ & & $s_{2}$ & & $s$ & & $\begin{array}{c}\varepsilon_{\min }-\varepsilon_{\max } \\
(\mathrm{MeV})\end{array}$ & Ref \\
\hline & & 15.27 & 2 & 5.62 & 8 & 1.407 & 15 & & & & & & & 1.407 & 15 & $12.0-18.9$ & 1976Le2 \\
\hline${ }^{128} \mathrm{Te}$ & 6 & 15.23 & 2 & 5.55 & 8 & 1.414 & 16 & & & & & & & 1.414 & 16 & $12.0-18.9$ & 1976Le2 \\
\hline${ }^{130} \mathrm{Te}$ & 6 & 15.21 & 2 & 5.19 & 8 & 1.377 & 15 & & & & & & & 1.377 & 15 & $12.0-18.9$ & 1976Le2 \\
\hline${ }^{127} \mathrm{I}$ & 4 & 15.05 & 2 & 4.76 & 4 & 0.979 & 12 & 17.33 & 8 & 1.83 & 29 & 0.035 & 8 & 1.014 & 14 & $12.0-20.0$ & 1999Bel \\
\hline & 4 & 14.64 & 22 & 4.00 & 27 & 0.860 & 179 & 16.51 & 39 & 3.21 & 91 & 0.215 & 166 & 1.075 & 244 & $12.2-20.0$ & 1989Ras \\
\hline & 4 & 14.98 & 20 & 4.93 & 27 & 1.228 & 158 & 17.03 & 35 & 2.65 & 169 & 0.106 & 125 & 1.334 & 201 & $8.8-19.8$ & 1969Be6 \\
\hline & 4 & 14.65 & 18 & 4.34 & 26 & 0.650 & 117 & 16.80 & 22 & 4.24 & 64 & 0.311 & 119 & 0.961 & 167 & $12.1-19.9$ & $1966 \mathrm{Br} 1$ \\
\hline & 5 & 14.62 & 37 & 4.31 & 53 & 0.760 & 279 & 16.76 & 43 & 4.30 & 107 & 0.397 & 279 & 1.157 & 395 & $12.1-19.9$ & 2006Var \\
\hline & 8 & 14.72 & 26 & 2.33 & 157 & 0.098 & 205 & 15.81 & 65 & 6.91 & 195 & 1.206 & 80 & 1.304 & 220 & $12.1-16.9$ & 1987Ber \\
\hline${ }^{133} \mathrm{Cs}$ & 4 & 15.44 & 1 & 5.50 & 2 & 1.397 & 5 & & & & & & & 1.397 & 5 & $12.0-19.0$ & 1974Le1 \\
\hline & 4 & 15.32 & 2 & 5.11 & 8 & 1.186 & 14 & & & & & & & 1.186 & 14 & $12.1-18.7$ & 1969Be1 \\
\hline & 5 & 15.19 & 14 & 4.95 & 53 & 1.267 & 83 & & & & & & & 1.267 & 83 & $12.1-19.0$ & 2016Va2 \\
\hline${ }^{138} \mathrm{Ba}$ & 5 & 15.16 & 9 & 4.68 & 35 & 1.150 & 52 & & & & & & & 1.150 & 52 & $12.1-18.7$ & 2016Va2 \\
\hline & 4 & 15.31 & 2 & 4.76 & 6 & 1.208 & 10 & & & & & & & 1.208 & 10 & $12.1-18.7$ & 1970Be8 \\
\hline${ }^{\text {nat }} \mathrm{Ba}$ & 4 & 15.46 & 1 & 5.57 & 4 & 1.511 & 8 & & & & & & & 1.511 & 8 & $10.1-17.8$ & 1971Be4 \\
\hline${ }^{139} \mathrm{La}$ & 4 & 15.30 & 3 & 5.11 & 10 & 1.436 & 18 & & & & & & & 1.436 & 18 & $12.0-18.9$ & 1972De1 \\
\hline & 4 & 15.15 & 1 & 4.09 & 4 & 1.068 & 8 & & & & & & & 1.068 & 8 & $12.0-18.9$ & 1971Be4 \\
\hline & 4 & 15.19 & 3 & 4.60 & 9 & 1.283 & 19 & & & & & & & 1.283 & 19 & $12.1-19.0$ & 1968Be5 \\
\hline & $4 a$ & 14.44 & 6 & 3.34 & 19 & 0.751 & 21 & & & & & & & 0.751 & 21 & $12.5-19.0$ & 1964Ric \\
\hline & $4 \mathrm{~b}$ & 14.48 & 12 & 3.18 & 33 & 0.741 & 48 & & & & & & & 0.741 & 48 & $12.5-19.0$ & 1964Ric \\
\hline${ }^{140} \mathrm{Ce}$ & 6 & 15.09 & 1 & 4.51 & 5 & 1.317 & 10 & & & & & & & 1.317 & 10 & $12.0-18.9$ & 1976Le2 \\
\hline${ }^{142} \mathrm{Ce}$ & 6 & 14.95 & 2 & 5.24 & 9 & 1.313 & 16 & & & & & & & 1.313 & 16 & $12.0-18.9$ & 1976Le2 \\
\hline${ }^{141} \mathrm{Pr}$ & 4 & 15.33 & 2 & 4.49 & 9 & 1.165 & 21 & & & & & & & 1.165 & 21 & $12.1-16.9$ & 1987Ber \\
\hline & 4 & 15.20 & 2 & 4.63 & 6 & 1.127 & 11 & & & & & & & 1.127 & 11 & $12.1-18.7$ & 1966Br1 \\
\hline & $4 a$ & 14.65 & 7 & 3.62 & 20 & 0.812 & 24 & & & & & & & 0.812 & 24 & $12.5-19.0$ & 1964Ric \\
\hline & $4 \mathrm{~b}$ & 14.74 & 13 & 3.59 & 39 & 0.816 & 60 & & & & & & & 0.816 & 60 & $12.5-19.0$ & 1964Ric \\
\hline & 12 & 15.42 & 2 & 4.39 & 5 & 1.081 & 10 & & & & & & & 1.081 & 10 & $12.0-18.1$ & 1972You \\
\hline & 12 & 15.17 & 1 & 4.88 & 3 & 1.262 & 6 & & & & & & & 1.262 & 6 & $12.0-16.9$ & 1971Be4 \\
\hline & 12 & 15.26 & 1 & 4.09 & 4 & 1.049 & 8 & & & & & & & 1.049 & 8 & $12.1-19.0$ & 1970Su1 \\
\hline & 13 & 15.43 & 2 & 4.02 & 7 & 1.081 & 13 & & & & & & & 1.081 & 13 & $12.0-18.9$ & 1972De1 \\
\hline${ }^{142} \mathrm{Nd}$ & $3 a$ & 15.02 & 4 & 4.41 & 18 & 1.205 & 30 & & & & & & & 1.205 & 30 & $13.1-18.0$ & 2003Var \\
\hline & $3 \mathrm{~b}$ & 15.04 & 12 & 4.50 & 49 & 1.221 & 111 & & & & & & & 1.221 & 111 & $13.1-18.0$ & 2003Var \\
\hline & 4 & 15.01 & 1 & 4.56 & 4 & 1.226 & 7 & & & & & & & 1.226 & 7 & $12.0-18.9$ & $1971 \mathrm{Ca} 1$ \\
\hline${ }^{143} \mathrm{Nd}$ & 4 & 15.08 & 2 & 4.99 & 9 & 1.281 & 16 & & & & & & & 1.281 & 16 & $12.0-19.0$ & $1971 \mathrm{Ca} 1$ \\
\hline${ }^{144} \mathrm{Nd}$ & 4 & 15.17 & 2 & 5.56 & 7 & 1.295 & 14 & & & & & & & 1.295 & 14 & $12.0-18.9$ & $1971 \mathrm{Ca} 1$ \\
\hline${ }^{145} \mathrm{Nd}$ & 4 & 15.14 & 5 & 6.75 & 22 & 1.456 & 36 & & & & & & & 1.456 & 36 & $12.0-18.9$ & $1971 \mathrm{Ca} 1$ \\
\hline${ }^{146} \mathrm{Nd}$ & 4 & 14.88 & 3 & 6.04 & 11 & 1.361 & 20 & & & & & & & 1.361 & 20 & $12.0-18.9$ & $1971 \mathrm{Ca} 1$ \\
\hline & $4 \mathrm{a}$ & 13.89 & 3 & 3.78 & 9 & 0.956 & 15 & & & & & & & 0.956 & 15 & $12.2-19.0$ & 1971Vas \\
\hline & $4 \mathrm{~b}$ & 13.94 & 5 & 3.98 & 13 & 0.996 & 30 & & & & & & & 0.996 & 30 & $12.2-19.0$ & 1971Vas \\
\hline${ }^{148} \mathrm{Nd}$ & 4 & 13.34 & 38 & 5.41 & 70 & 0.623 & 184 & 15.79 & 11 & 4.57 & 56 & 0.552 & 165 & 1.175 & 247 & $10.8-18.6$ & $1971 \mathrm{Ca} 1$ \\
\hline & 4 & 13.73 & 3 & 2.71 & 18 & 0.870 & 50 & 15.96 & 4 & 1.09 & 18 & 0.113 & 23 & 0.982 & 55 & $13.0-17.0$ & 1969Vas \\
\hline${ }^{150} \mathrm{Nd}$ & 4 & 12.49 & 9 & 3.93 & 29 & 0.556 & 55 & 16.23 & 7 & 4.85 & 34 & 0.696 & 65 & 1.252 & 85 & $10.8-18.6$ & 1971Ca1 \\
\hline${ }^{144} \mathrm{Sm}$ & 4 & 15.37 & 2 & 4.53 & 7 & 1.274 & 15 & & & & & & & 1.274 & 15 & $12.1-18.9$ & $1974 \mathrm{Ca} 5$ \\
\hline${ }^{148} \mathrm{Sm}$ & 4 & 14.91 & 1 & 5.15 & 6 & 1.260 & 10 & & & & & & & 1.260 & 10 & $12.1-18.9$ & 1974Ca5 \\
\hline & $4 \mathrm{a}$ & 14.02 & 2 & 4.04 & 5 & 0.947 & 11 & & & & & & & 0.947 & 11 & $7.7-19.0$ & 1971Vas \\
\hline & $4 \mathrm{~b}$ & 14.17 & 4 & 4.38 & 8 & 1.029 & 19 & & & & & & & 1.029 & 19 & $7.7-19.0$ & 1971Vas \\
\hline${ }^{150} \mathrm{Sm}$ & 4 & 14.76 & 3 & 6.01 & 12 & 1.340 & 20 & & & & & & & 1.340 & 20 & $12.1-18.9$ & $1974 \mathrm{Ca} 5$ \\
\hline & 4 & 13.99 & 3 & 4.40 & 11 & 1.137 & 19 & & & & & & & 1.137 & 19 & $12.2-19.0$ & 1969Vas \\
\hline${ }^{152} \mathrm{Sm}$ & 4 & 12.56 & 4 & 3.53 & 13 & 0.511 & 25 & 15.97 & 3 & 4.77 & 14 & 0.704 & 30 & 1.215 & 39 & $10.9-18.8$ & 1974Ca5 \\
\hline & 4 & 11.74 & 2 & 2.24 & 8 & 0.751 & 23 & 15.00 & 3 & 2.31 & 10 & 0.672 & 24 & 1.423 & 33 & $11.0-18.8$ & 1969Vas \\
\hline${ }^{154} \mathrm{Sm}$ & 0 & 12.31 & 29 & 3.27 & 1.04 & 0.483 & 217 & 15.95 & 36 & 5.53 & 176 & 0.729 & 305 & 1.212 & 374 & $10.9-18.6$ & $1981 \mathrm{Gur}$ \\
\hline & 4 & 12.42 & 4 & 3.43 & 16 & 0.495 & 30 & 16.20 & 4 & 5.29 & 22 & 0.716 & 39 & 1.211 & 49 & $11.0-18.6$ & $1974 \mathrm{Ca} 5$ \\
\hline & 4 & 11.34 & 4 & 2.09 & 15 & 0.322 & 23 & 14.98 & 3 & 3.86 & 9 & 0.926 & 23 & 1.248 & 32 & $11.0-18.8$ & 1969Vas \\
\hline${ }^{151} \mathrm{Eu}$ & $4 a$ & 13.43 & 34 & 5.18 & 31 & 0.623 & 163 & 14.52 & 7 & 2.28 & 60 & 0.258 & 133 & 0.880 & 210 & $10.2-18.0$ & 1971Vas \\
\hline & $4 \mathrm{~b}$ & 13.27 & 68 & 5.04 & 67 & 0.545 & 285 & 14.54 & 7 & 2.59 & 80 & 0.327 & 226 & 0.872 & 364 & $10.2-18.0$ & 1971Vas \\
\hline${ }^{153} \mathrm{Eu}$ & 4 & 12.47 & 5 & 3.26 & 18 & 0.416 & 31 & 16.07 & 5 & 5.48 & 21 & 0.769 & 42 & 1.185 & 52 & $10.9-18.7$ & 1969Be8 \\
\hline & $4 a$ & 11.99 & 3 & 2.19 & 11 & 0.282 & 13 & 15.26 & 4 & 3.12 & 13 & 0.536 & 18 & 0.819 & 22 & $11.0-18.8$ & 1971Vas \\
\hline & $4 \mathrm{~b}$ & 12.00 & 3 & 2.23 & 11 & 0.292 & 14 & 15.19 & 6 & 2.88 & 17 & 0.496 & 29 & 0.788 & 33 & $11.0-18.8$ & 1971Vas \\
\hline${ }^{152} \mathrm{Gd}$ & $4 \mathrm{a}$ & 11.96 & 6 & 3.49 & 18 & 0.397 & 26 & 14.75 & 4 & 2.87 & 14 & 0.475 & 26 & 0.872 & 37 & $10.2-18.0$ & 1971Vas \\
\hline & $4 \mathrm{~b}$ & 11.96 & 6 & 3.49 & 18 & 0.396 & 27 & 14.75 & 5 & 2.88 & 17 & 0.477 & 32 & 0.873 & 42 & $10.2-18.0$ & 1971Vas \\
\hline${ }^{154} \mathrm{Gd}$ & $4 \mathrm{a}$ & 12.09 & 5 & 3.08 & 17 & 0.380 & 23 & 15.10 & 4 & 3.09 & 15 & 0.493 & 27 & 0.873 & 35 & $10.2-18.0$ & 1971Vas \\
\hline & $4 \mathrm{~b}$ & 12.11 & 5 & 3.11 & 17 & 0.389 & 24 & 15.07 & 5 & 2.93 & 18 & 0.467 & 33 & 0.856 & 41 & $10.2-18.0$ & 1971Vas \\
\hline${ }^{156} \mathrm{Gd}$ & 0 & 12.63 & 24 & 3.61 & 80 & 0.632 & 177 & 15.97 & 23 & 4.10 & 105 & 0.537 & 191 & 1.169 & 260 & $10.9-18.7$ & $1981 \mathrm{Gur}$ \\
\hline & $4 \mathrm{a}$ & 12.00 & 4 & 3.50 & 17 & 0.501 & 25 & 15.00 & 4 & 2.06 & 11 & 0.343 & 19 & 0.844 & 31 & $11.0-18.8$ & 1971Vas \\
\hline & $4 \mathrm{~b}$ & 12.01 & 6 & 4.19 & 37 & 0.583 & 52 & 15.02 & 6 & 2.09 & 20 & 0.322 & 39 & 0.905 & 65 & $11.0-18.8$ & 1971Vas \\
\hline${ }^{158} \mathrm{Gd}$ & $4 \mathrm{a}$ & 12.00 & 5 & 3.37 & 18 & 0.449 & 26 & 15.19 & 5 & 2.97 & 19 & 0.473 & 27 & 0.921 & 38 & $10.2-18.0$ & 1971Vas \\
\hline & $4 \mathrm{~b}$ & 12.06 & 6 & 3.50 & 18 & 0.487 & 29 & 15.02 & 9 & 2.21 & 26 & 0.368 & 45 & 0.855 & 54 & $10.2-18.0$ & 1971Vas \\
\hline
\end{tabular}


Table 2 (continued)

\begin{tabular}{|c|c|c|c|c|c|c|c|c|c|c|c|c|c|c|c|c|c|}
\hline \multirow{2}{*}{$\begin{array}{c}\text { Nucl } \\
{ }^{160} \mathrm{Gd}\end{array}$} & \multirow{2}{*}{$\frac{\text { Id }}{3 \mathrm{a}}$} & \multicolumn{2}{|c|}{$\begin{array}{c}E_{r, 1} \\
(\mathrm{MeV})\end{array}$} & \multicolumn{2}{|c|}{$\begin{array}{c}\Gamma_{r, 1} \\
(\mathrm{MeV})\end{array}$} & \multicolumn{2}{|l|}{$s_{1}$} & \multicolumn{2}{|c|}{$\begin{array}{c}E_{r, 2} \\
(\mathrm{MeV})\end{array}$} & $\begin{array}{r}\Gamma_{r} \\
(\mathrm{Me}\end{array}$ & & $s_{2}$ & & $s$ & & $\begin{array}{c}\varepsilon_{\min }-\varepsilon_{\max } \\
(\mathrm{MeV})\end{array}$ & Ref \\
\hline & & 12.47 & 14 & 3.85 & 49 & 0.678 & 101 & 16.26 & 11 & 4.69 & 48 & 0.700 & 104 & 1.378 & 145 & $10.9-18.8$ & 2003Var \\
\hline & $3 b$ & 12.42 & 16 & 3.71 & 67 & 0.638 & 142 & 16.25 & 16 & 5.07 & 88 & 0.764 & 177 & 1.402 & 227 & $10.9-18.8$ & 2003Var \\
\hline & 4 & 12.34 & 6 & 3.13 & 24 & 0.502 & 43 & 16.16 & 6 & 4.99 & 29 & 0.717 & 54 & 1.219 & 69 & $10.9-18.7$ & 1969Be8 \\
\hline${ }^{159} \mathrm{~Tb}$ & 4 & 12.55 & 5 & 3.23 & 15 & 0.452 & 33 & 16.16 & 5 & 5.74 & 22 & 1.059 & 50 & 1.511 & 60 & $11.1-19.0$ & 1976Gor \\
\hline & 4 & 12.22 & 6 & 3.37 & 28 & 0.497 & 48 & 16.05 & 6 & 4.79 & 29 & 0.737 & 57 & 1.234 & 75 & $11.0-18.7$ & 1968Be5 \\
\hline & 4 & 12.33 & 5 & 3.04 & 18 & 0.414 & 31 & 15.85 & 6 & 4.60 & 29 & 0.631 & 46 & 1.045 & 55 & $10.8-18.7$ & $1964 \mathrm{Br} 1$ \\
\hline & $4 a$ & 12.58 & 7 & 3.53 & 21 & 0.749 & 44 & 16.14 & 11 & 1.81 & 19 & 0.362 & 32 & 1.111 & 55 & $10.8-18.8$ & 1962Bog \\
\hline & $4 \mathrm{~b}$ & 12.46 & 10 & 3.52 & 33 & 0.687 & 66 & 16.10 & 11 & 2.93 & 31 & 0.539 & 58 & 1.225 & 88 & $10.8-18.8$ & 1962Bog \\
\hline & $4 a$ & 12.43 & 7 & 3.12 & 23 & 0.530 & 48 & 16.42 & 11 & 4.70 & 48 & 0.850 & 90 & 1.380 & 102 & $11.0-18.0$ & $1958 \mathrm{Ful}$ \\
\hline & $4 \mathrm{~b}$ & 12.43 & 7 & 3.12 & 23 & 0.529 & 48 & 16.42 & 12 & 4.72 & 49 & 0.854 & 92 & 1.383 & 104 & $11.0-18.0$ & 1958Ful \\
\hline & 5 & 12.16 & 6 & 3.50 & 28 & 0.450 & 49 & 15.73 & 7 & 5.72 & 30 & 0.766 & 59 & 1.216 & 77 & $11.1-19.0$ & 2011Var \\
\hline${ }^{165} \mathrm{Ho}$ & 0 & 12.47 & 13 & 2.84 & 59 & 0.445 & 100 & 15.59 & 18 & 3.78 & 72 & 0.520 & 120 & 0.965 & 156 & $11.1-18.7$ & $1981 \mathrm{Gur}$ \\
\hline & 0 & 12.62 & 14 & 3.02 & 58 & 0.501 & 109 & 15.47 & 15 & 3.42 & 62 & 0.492 & 117 & 0.994 & 160 & $11.1-18.7$ & 1976Gu2 \\
\hline & 4 & 12.38 & 2 & 2.92 & 9 & 0.453 & 17 & 15.98 & 3 & 4.66 & 14 & 0.692 & 23 & 1.145 & 29 & $10.9-18.7$ & 1969Be8 \\
\hline & 4 & 12.11 & 4 & 2.81 & 19 & 0.489 & 38 & 15.78 & 5 & 4.83 & 24 & 0.856 & 50 & 1.345 & 63 & $11.0-18.7$ & 1968Be5 \\
\hline & 4 & 11.91 & 3 & 2.61 & 15 & 0.435 & 29 & 16.38 & 4 & 5.79 & 26 & 1.343 & 62 & 1.778 & 69 & $11.0-18.3$ & 1966Axe \\
\hline & $4 a$ & 12.41 & 5 & 2.99 & 14 & 0.421 & 30 & 16.51 & 5 & 6.20 & 31 & 1.153 & 63 & 1.574 & 69 & $10.8-18.8$ & 1976Gor \\
\hline & $4 \mathrm{~b}$ & 12.41 & 5 & 2.99 & 14 & 0.421 & 30 & 16.51 & 5 & 6.20 & 31 & 1.153 & 63 & 1.574 & 69 & $10.8-18.8$ & 1976Gor \\
\hline${ }^{168} \mathrm{Er}$ & 0 & 12.33 & 36 & 4.18 & 140 & 0.698 & 281 & 15.65 & 20 & 3.58 & 96 & 0.516 & 225 & 1.214 & 360 & $10.9-18.8$ & 1981Gur \\
\hline${ }^{174} \mathrm{Yb}$ & 0 & 12.73 & 25 & 3.95 & 69 & 0.909 & 211 & 15.80 & 18 & 3.18 & 81 & 0.496 & 186 & 1.405 & 281 & $10.9-18.7$ & 1981Gur \\
\hline${ }^{175} \mathrm{Lu}$ & 4 & 12.44 & 9 & 2.99 & 30 & 0.456 & 59 & 15.65 & 9 & 4.32 & 34 & 0.699 & 71 & 1.155 & 92 & $11.0-18.7$ & 1969Be6 \\
\hline${ }^{176} \mathrm{Hf}$ & 4 & 12.46 & 4 & 3.13 & 13 & 0.585 & 31 & 15.86 & 5 & 4.44 & 18 & 0.677 & 38 & 1.262 & 49 & $10.9-17.9$ & 1977Gor \\
\hline${ }^{178} \mathrm{Hf}$ & 0 & 12.59 & 22 & 4.95 & 68 & 1.126 & 190 & 15.65 & 16 & 3.00 & 61 & 0.401 & 128 & 1.527 & 229 & $10.8-18.6$ & 1981Gur \\
\hline & 0 & 12.40 & 18 & 3.95 & 37 & 0.879 & 123 & 15.56 & 16 & 3.47 & 57 & 0.556 & 124 & 1.435 & 175 & $8.1-18.6$ & $1976 \mathrm{Gu} 2$ \\
\hline & 4 & 12.57 & 5 & 3.26 & 17 & 0.643 & 39 & 15.91 & 5 & 3.71 & 19 & 0.564 & 40 & 1.207 & 56 & $11.0-17.9$ & 1977Gor \\
\hline & 4 & 12.68 & 18 & 2.78 & 56 & 0.248 & 127 & 15.14 & 29 & 6.51 & 40 & 1.077 & 167 & 1.325 & 209 & $11.0-18.8$ & 1976Gor \\
\hline${ }^{180} \mathrm{Hf}$ & 0 & 12.74 & 31 & 4.93 & 88 & 1.089 & 256 & 15.59 & 18 & 3.04 & 79 & 0.407 & 177 & 1.496 & 311 & $10.8-18.7$ & 1981Gur \\
\hline & 0 & 12.45 & 21 & 3.64 & 43 & 0.769 & 143 & 15.46 & 19 & 3.68 & 68 & 0.630 & 160 & 1.399 & 215 & $8.6-18.7$ & $1976 \mathrm{Gu} 2$ \\
\hline & 4 & 12.56 & 4 & 2.96 & 12 & 0.584 & 26 & 15.86 & 3 & 3.51 & 13 & 0.558 & 27 & 1.142 & 37 & $11.0-17.9$ & 1977Gor \\
\hline${ }^{181} \mathrm{Ta}$ & 0 & 12.36 & 39 & 3.41 & 110 & 0.638 & 331 & 15.26 & 40 & 4.71 & 111 & 0.774 & 356 & 1.412 & 486 & $10.8-18.6$ & 1981Gur \\
\hline & 0 & 12.36 & 39 & 3.41 & 110 & 0.638 & 331 & 15.26 & 40 & 4.71 & 111 & 0.774 & 356 & 1.412 & 486 & $10.8-18.6$ & $1976 \mathrm{Gu} 2$ \\
\hline & $3 a$ & 12.52 & 11 & 3.23 & 23 & 0.582 & 62 & 15.47 & 10 & 3.89 & 27 & 0.680 & 67 & 1.262 & 91 & $10.0-18.8$ & 2003Var \\
\hline & $3 \mathrm{~b}$ & 12.45 & 17 & 3.08 & 50 & 0.536 & 124 & 15.43 & 16 & 4.17 & 71 & 0.742 & 165 & 1.278 & 206 & $10.0-18.8$ & 2003Var \\
\hline & 4 & 12.65 & 2 & 2.91 & 5 & 0.558 & 12 & 15.61 & 2 & 3.53 & 7 & 0.646 & 15 & 1.203 & 19 & $8-18.8$ & 2001Bel \\
\hline & 4 & 12.43 & 6 & 2.90 & 22 & 0.518 & 50 & 15.42 & 6 & 4.03 & 21 & 0.743 & 56 & 1.261 & 75 & $11.0-18.7$ & 1968Be5 \\
\hline & 4 & 12.52 & 8 & 1.90 & 41 & 0.164 & 69 & 15.06 & 20 & 5.83 & 72 & 0.917 & 140 & 1.081 & 156 & $10.8-18.7$ & 1963Br1 \\
\hline & 4 & 13.23 & 14 & 5.09 & 40 & 1.283 & 106 & 15.74 & 3 & 1.47 & 22 & 0.257 & 48 & 1.540 & 116 & $11.0-18.5$ & 1962Bog \\
\hline & $4 a$ & 11.97 & 7 & 2.78 & 35 & 0.473 & 59 & 15.16 & 7 & 3.68 & 22 & 0.623 & 53 & 1.096 & 79 & $10.8-18.8$ & 1969Ish \\
\hline & $4 b$ & 12.03 & 8 & 2.74 & 39 & 0.493 & 67 & 15.17 & 7 & 3.11 & 28 & 0.557 & 62 & 1.050 & 91 & $10.8-18.8$ & 1969Ish \\
\hline & $4 a$ & 12.45 & 28 & 3.52 & 91 & 0.626 & 174 & 15.66 & 23 & 3.17 & 74 & 0.511 & 159 & 1.138 & 235 & $11.0-18.0$ & 1967Ant \\
\hline & $4 \mathrm{~b}$ & 12.36 & 39 & 3.34 & 145 & 0.569 & 288 & 15.60 & 37 & 3.67 & 162 & 0.591 & 316 & 1.160 & 427 & $11.0-18.0$ & 1967Ant \\
\hline & $4 a$ & 12.40 & 3 & 2.62 & 12 & 0.517 & 30 & 15.66 & 5 & 4.81 & 19 & 0.962 & 46 & 1.479 & 55 & $11.0-18.7$ & $1958 \mathrm{Ful}$ \\
\hline & $4 \mathrm{~b}$ & 12.41 & 3 & 2.67 & 14 & 0.529 & 33 & 15.67 & 5 & 4.77 & 21 & 0.948 & 49 & 1.478 & 59 & $11.0-18.7$ & 1958Ful \\
\hline & 5 & 12.68 & 34 & 3.74 & 111 & 0.711 & 285 & 15.56 & 25 & 3.95 & 79 & 0.522 & 245 & 1.233 & 376 & $11.5-19.0$ & 2012 Var \\
\hline${ }^{182} \mathrm{~W}$ & 0 & 13.08 & 34 & 7.29 & 100 & 1.655 & 268 & 15.20 & 21 & 1.53 & 123 & 0.091 & 90 & 1.746 & 283 & $11.0-18.8$ & 1981Gur \\
\hline & 0 & 12.00 & 27 & 3.13 & 59 & 0.555 & 196 & 14.96 & 32 & 5.06 & 108 & 0.839 & 267 & 1.394 & 331 & $8.5-18.8$ & 1976Gu2 \\
\hline & 4 & 12.80 & 5 & 3.17 & 12 & 0.629 & 35 & 15.71 & 5 & 4.14 & 14 & 0.704 & 39 & 1.333 & 52 & $10.8-18.6$ & 1978Go3 \\
\hline${ }^{184} \mathrm{~W}$ & 0 & 12.27 & 45 & 5.17 & 188 & 1.144 & 513 & 15.10 & 17 & 3.15 & 143 & 0.347 & 301 & 1.491 & 595 & $11.0-17.6$ & 1981Gur \\
\hline & 4 & 13.23 & 7 & 4.61 & 11 & 0.990 & 46 & 15.75 & 5 & 3.46 & 18 & 0.416 & 41 & 1.406 & 62 & $8.0-18.6$ & 1978Go3 \\
\hline & $4 a$ & 12.56 & 8 & 3.27 & 20 & 0.557 & 65 & 15.94 & 13 & 5.37 & 56 & 0.959 & 107 & 1.516 & 125 & $11.0-18.8$ & 1973Gor \\
\hline & $4 \mathrm{~b}$ & 12.62 & 9 & 3.43 & 22 & 0.625 & 80 & 15.92 & 20 & 4.48 & 91 & 0.810 & 173 & 1.435 & 191 & $11.0-18.8$ & 1973Gor \\
\hline${ }^{186} \mathrm{~W}$ & 0 & 13.01 & 48 & 6.25 & 93 & 1.400 & 392 & 14.81 & 30 & 2.84 & 186 & 0.205 & 255 & 1.605 & 468 & $10.9-18.7$ & 1981Gur \\
\hline & 4 & 12.73 & 7 & 3.06 & 16 & 0.557 & 51 & 15.34 & 6 & 4.33 & 17 & 0.785 & 58 & 1.342 & 77 & $11.0-17.8$ & 1978Go3 \\
\hline & 4 & 12.74 & 5 & 2.94 & 12 & 0.484 & 38 & 15.25 & 6 & 4.61 & 17 & 0.755 & 47 & 1.239 & 60 & $10.9-18.7$ & 1969Be8 \\
\hline & $4 \mathrm{a}$ & 12.87 & 13 & 3.82 & 31 & 0.734 & 112 & 15.92 & 22 & 4.96 & 66 & 0.658 & 133 & 1.392 & 173 & $11.0-18.8$ & 1973Gor \\
\hline & $4 \mathrm{~b}$ & 12.88 & 14 & 3.83 & 32 & 0.749 & 132 & 15.88 & 29 & 4.60 & 138 & 0.620 & 215 & 1.369 & 253 & $11.0-18.8$ & 1973Gor \\
\hline${ }^{185} \mathrm{Re}$ & $4 \mathrm{a}$ & 12.89 & 6 & 3.48 & 12 & 0.674 & 52 & 15.84 & 10 & 4.37 & 55 & 0.649 & 83 & 1.324 & 98 & $10.2-18.0$ & 1973Gor \\
\hline & $4 \mathrm{~b}$ & 12.62 & 4 & 2.00 & 19 & 0.213 & 37 & 15.63 & 36 & 9.15 & 57 & 1.727 & 142 & 1.940 & 147 & $10.2-18.0$ & 1973Gor \\
\hline${ }^{\text {nat }} \mathrm{Re}$ & 4 & 14.30 & 1 & 7.05 & 5 & 1.902 & 11 & & & & & & & 1.902 & 11 & $10.2-18.0$ & 1975Vey \\
\hline${ }^{186} \mathrm{Os}$ & 5 & 13.00 & 44 & 3.09 & 79 & 0.557 & 283 & 15.09 & 35 & 3.39 & 58 & 0.542 & 275 & 1.100 & 394 & $11.1-18.9$ & 2015Var \\
\hline & 7 & 13.27 & 13 & 3.65 & 26 & 0.756 & 95 & 15.39 & 8 & 2.80 & 31 & 0.394 & 83 & 1.150 & 126 & $11.1-18.7$ & 1979Be4 \\
\hline${ }^{188} \mathrm{Os}$ & 5 & 13.14 & 43 & 3.59 & 68 & 0.794 & 319 & 15.22 & 30 & 2.98 & 76 & 0.507 & 292 & 1.301 & 432 & $10.8-18.9$ & 2014Var \\
\hline & 9 & 13.12 & 7 & 3.61 & 12 & 0.739 & 59 & 15.17 & 6 & 3.55 & 19 & 0.597 & 61 & 1.336 & 85 & $10.8-18.7$ & 1979Be4 \\
\hline${ }^{189} \mathrm{Os}$ & 5 & 13.18 & 26 & 3.64 & 40 & 0.873 & 201 & 15.32 & 25 & 2.95 & 63 & 0.365 & 180 & 1.238 & 270 & $10.8-18.9$ & 2014Var \\
\hline & 9 & 12.93 & 7 & 3.39 & 12 & 0.657 & 53 & 14.86 & 5 & 3.19 & 16 & 0.582 & 55 & 1.239 & 76 & $10.8-18.7$ & 1979Be4 \\
\hline${ }^{190} \mathrm{Os}$ & 5 & 13.21 & 33 & 3.59 & 46 & 0.881 & 256 & 15.28 & 36 & 2.93 & 79 & 0.348 & 233 & 1.229 & 346 & $10.8-18.9$ & 2015Var \\
\hline & 9 & 13.04 & 10 & 3.62 & 14 & 0.694 & 85 & 14.75 & 8 & 3.51 & 20 & 0.549 & 85 & 1.243 & 120 & $10.8-18.7$ & 1979Be4 \\
\hline
\end{tabular}


Table 2 (continued)

\begin{tabular}{|c|c|c|c|c|c|c|c|c|c|c|c|c|c|c|c|c|c|}
\hline \multirow{2}{*}{$\begin{array}{c}\text { Nucl } \\
{ }^{192} \mathrm{Os}\end{array}$} & \multirow{2}{*}{$\begin{array}{l}\text { Id } \\
5\end{array}$} & \multicolumn{2}{|c|}{$\begin{array}{c}E_{r, 1} \\
(\mathrm{MeV})\end{array}$} & \multicolumn{2}{|c|}{$\begin{array}{c}\Gamma_{r, 1} \\
(\mathrm{MeV}) \\
\end{array}$} & \multicolumn{2}{|l|}{$s_{1}$} & \multicolumn{2}{|c|}{$\begin{array}{c}E_{r, 2} \\
(\mathrm{MeV}) \\
\end{array}$} & $\begin{array}{r}\Gamma_{r, 2} \\
(\mathrm{MeI} \\
\end{array}$ & & $s_{2}$ & & $s$ & & $\begin{array}{c}\varepsilon_{\min }-\varepsilon_{\max } \\
(\mathrm{MeV})\end{array}$ & Ref \\
\hline & & 13.08 & 68 & 3.61 & 104 & 0.752 & 558 & 14.98 & 56 & 3.68 & 110 & 0.523 & 534 & 1.275 & 772 & $10.8-18.9$ & 2015Var \\
\hline & 9 & 13.04 & 10 & 3.62 & 14 & 0.690 & 85 & 14.75 & 8 & 3.51 & 20 & 0.545 & 84 & 1.235 & 120 & $10.8-18.7$ & 1979Be4 \\
\hline${ }^{191} \mathrm{Ir}$ & 4 & 13.16 & 15 & 3.75 & 25 & 0.894 & 155 & 15.24 & 22 & 3.69 & 97 & 0.359 & 171 & 1.253 & 231 & $11.0-16.8$ & 1978Go1 \\
\hline & 4 & 12.92 & 7 & 2.16 & 39 & 0.278 & 91 & 16.40 & 46 & 10.48 & 111 & 2.304 & 292 & 2.582 & 305 & $11.1-16.9$ & 1978Go2 \\
\hline${ }^{193} \mathrm{Ir}$ & 4 & 12.85 & 8 & 1.64 & 37 & 0.149 & 64 & 14.18 & 16 & 5.94 & 37 & 1.322 & 65 & 1.471 & 91 & $11.0-16.8$ & 1978Go1 \\
\hline nat Ir & 4 & 13.81 & 1 & 4.97 & 4 & 1.386 & 8 & & & & & & & 1.386 & 8 & $10.2-18.0$ & 1975Vey \\
\hline${ }^{194} \mathrm{Pt}$ & 4 & 13.66 & 7 & 4.29 & 13 & 1.203 & 69 & 16.70 & 42 & 4.55 & 264 & 0.088 & 81 & 1.291 & 106 & $11.0-17.8$ & 1978Go1 \\
\hline & 13 & 13.39 & 7 & 3.73 & 16 & 0.953 & 70 & 16.58 & 9 & 4.69 & 55 & 0.603 & 97 & 1.557 & 119 & $11.2-18.0$ & 1978Go2 \\
\hline${ }^{195} \mathrm{Pt}$ & 4 & 13.28 & 12 & 3.71 & 22 & 0.964 & 126 & 15.44 & 24 & 3.56 & 96 & 0.273 & 132 & 1.237 & 182 & $11.0-17.8$ & 1978Go1 \\
\hline & 13 & 13.24 & 9 & 3.44 & 21 & 0.847 & 106 & 16.26 & 17 & 4.78 & 99 & 0.716 & 181 & 1.563 & 209 & $11.2-18.0$ & $1978 \mathrm{Go} 2$ \\
\hline${ }^{196} \mathrm{Pt}$ & 4 & 13.38 & 7 & 2.87 & 39 & 0.376 & 149 & 14.18 & 19 & 6.94 & 71 & 1.067 & 130 & 1.443 & 198 & $11.0-17.8$ & 1978Go1 \\
\hline${ }^{198} \mathrm{Pt}$ & 4 & 13.62 & 2 & 4.83 & 7 & 1.426 & 12 & & & & & & & 1.426 & 12 & $11.0-17.8$ & 1978Go1 \\
\hline${ }^{197} \mathrm{Au}$ & 0 & 13.72 & 7 & 5.43 & 30 & 1.570 & 69 & & & & & & & 1.570 & 69 & $11.1-17.0$ & 1981Gur \\
\hline & 0 & 13.68 & 7 & 5.45 & 23 & 1.580 & 54 & & & & & & & 1.580 & 54 & $8.2-17.0$ & 1976Gu2 \\
\hline & 4 & 13.81 & 2 & 4.79 & 8 & 1.410 & 19 & & & & & & & 1.410 & 19 & $11.0-16.8$ & 1970Ve1 \\
\hline & 4 & 13.88 & 3 & 4.09 & 9 & 1.231 & 22 & & & & & & & 1.231 & 22 & $11.1-16.8$ & $1962 \mathrm{Fu} 2$ \\
\hline & $4 \mathrm{a}$ & 13.28 & 3 & 4.44 & 14 & 1.346 & 29 & & & & & & & 1.346 & 29 & $11.1-17.0$ & 1973Sor \\
\hline & $4 \mathrm{~b}$ & 13.36 & 5 & 4.95 & 24 & 1.460 & 52 & & & & & & & 1.460 & 52 & $11.1-17.0$ & 1973Sor \\
\hline & $4 \mathrm{a}$ & 13.77 & 3 & 4.72 & 12 & 1.475 & 26 & & & & & & & 1.475 & 26 & $11.4-17.0$ & $1958 \mathrm{Ful}$ \\
\hline & $4 \mathrm{~b}$ & 13.78 & 3 & 4.76 & 12 & 1.485 & 28 & & & & & & & 1.485 & 28 & $11.4-17.0$ & $1958 \mathrm{Ful}$ \\
\hline & 8 & 13.86 & 3 & 4.94 & 15 & 1.354 & 34 & & & & & & & 1.354 & 34 & $12.1-16.9$ & 1987Ber \\
\hline & $8 \mathrm{a}$ & 13.66 & 6 & 4.21 & 22 & 1.272 & 38 & & & & & & & 1.272 & 38 & $11.0-16.8$ & 2010Var \\
\hline & $8 \mathrm{~b}$ & 13.70 & 14 & 4.36 & 46 & 1.295 & 104 & & & & & & & 1.295 & 104 & $11.0-16.8$ & 2010Var \\
\hline${ }^{203} \mathrm{Tl}$ & 4 & 14.06 & 8 & 3.95 & 21 & 0.926 & 39 & & & & & & & 0.926 & 39 & $9.0-17.9$ & 1970Ant \\
\hline${ }^{205} \mathrm{Tl}$ & 4 & 14.47 & 5 & 2.93 & 16 & 0.756 & 27 & & & & & & & 0.756 & 27 & $10.5-17.9$ & 1970Ant \\
\hline${ }^{206} \mathrm{~Pb}$ & 4 & 13.61 & 1 & 4.01 & 5 & 1.072 & 9 & & & & & & & 1.072 & 9 & $10.0-17.0$ & $1964 \mathrm{Ha} 2$ \\
\hline & $4 a$ & 13.57 & 2 & 5.44 & 6 & 1.271 & 14 & & & & & & & 1.271 & 14 & $10.1-16.9$ & 1973Sor \\
\hline & $4 \mathrm{~b}$ & 13.80 & 3 & 6.09 & 9 & 1.422 & 22 & & & & & & & 1.422 & 22 & $10.1-16.9$ & 1973Sor \\
\hline${ }^{207} \mathrm{~Pb}$ & 4 & 13.57 & 2 & 4.22 & 6 & 1.042 & 10 & & & & & & & 1.042 & 10 & $10.0-17.0$ & $1964 \mathrm{Ha} 2$ \\
\hline${ }^{208} \mathrm{~Pb}$ & $3 a$ & 13.34 & 3 & 3.64 & 8 & 1.270 & 15 & & & & & & & 1.270 & 15 & $10.9-18.8$ & 2003Var \\
\hline & $3 \mathrm{~b}$ & 13.43 & 7 & 3.83 & 18 & 1.301 & 44 & & & & & & & 1.301 & 44 & $10.9-18.8$ & 2003Var \\
\hline & 4 & 13.52 & 1 & 3.47 & 3 & 1.091 & 6 & & & & & & & 1.091 & 6 & $10.8-18.8$ & 1995Bel \\
\hline & 4 & 13.52 & 2 & 4.67 & 5 & 1.473 & 14 & & & & & & & 1.473 & 14 & $10.2-16.8$ & 1970Ve1 \\
\hline & 4 & 13.50 & 1 & 4.15 & 5 & 1.048 & 9 & & & & & & & 1.048 & 9 & $10.0-17.0$ & $1964 \mathrm{Ha} 2$ \\
\hline & $4 a$ & 13.21 & 1 & 4.37 & 4 & 1.127 & 10 & & & & & & & 1.127 & 10 & $10.9-18.6$ & 1973Sor \\
\hline & $4 \mathrm{~b}$ & 13.55 & 3 & 5.56 & 9 & 1.420 & 24 & & & & & & & 1.420 & 24 & $10.9-18.6$ & 1973Sor \\
\hline & 5 & 13.41 & 3 & 4.42 & 10 & 1.397 & 24 & & & & & & & 1.397 & 24 & $10.5-17.1$ & 2013Ish \\
\hline & 12 & 13.79 & 2 & 4.49 & 6 & 1.488 & 19 & & & & & & & 1.488 & 19 & $10.0-14.9$ & 1972You \\
\hline${ }^{\text {nat }} \mathrm{Pb}$ & 2 & 13.58 & 2 & 4.05 & 7 & 1.356 & 19 & & & & & & & 1.356 & 19 & $12.1-16.9$ & 1985Ahr \\
\hline & 8 & 13.64 & 2 & 3.74 & 9 & 1.209 & 24 & & & & & & & 1.209 & 24 & $12.1-16.9$ & 1987Ber \\
\hline${ }^{209} \mathrm{Bi}$ & 0 & 13.87 & 8 & 5.04 & 31 & 1.559 & 70 & & & & & & & 1.559 & 70 & $10.9-18.3$ & $1976 \mathrm{Gu} 2$ \\
\hline & 4 & 13.35 & 1 & 3.75 & 2 & 1.023 & 4 & & & & & & & 1.023 & 4 & $10.8-18.8$ & 1995Bel \\
\hline & 4 & 13.49 & 1 & 4.28 & 5 & 1.132 & 8 & & & & & & & 1.132 & 8 & $10.0-17.0$ & $1964 \mathrm{Ha} 2$ \\
\hline & $4 \mathrm{a}$ & 13.04 & 3 & 3.93 & 10 & 1.212 & 19 & & & & & & & 1.212 & 19 & $10.9-18.6$ & 1973Sor \\
\hline & $4 \mathrm{~b}$ & 13.19 & 5 & 4.70 & 19 & 1.405 & 43 & & & & & & & 1.405 & 43 & $10.9-18.6$ & 1973Sor \\
\hline & $4 \mathrm{a}$ & 12.89 & 9 & 3.28 & 16 & 1.204 & 33 & & & & & & & 1.204 & 33 & $11.0-18.5$ & 1967Ant \\
\hline & $4 \mathrm{~b}$ & 13.06 & 17 & 3.56 & 32 & 1.251 & 85 & & & & & & & 1.251 & 85 & $11.0-18.5$ & 1967Ant \\
\hline & 5 & 13.50 & 6 & 4.28 & 20 & 1.137 & 29 & & & & & & & 1.137 & 29 & $10.0-18.9$ & 2016Va2 \\
\hline & 12 & 13.66 & 2 & 4.17 & 5 & 1.387 & 13 & & & & & & & 1.387 & 13 & $10.0-14.8$ & 1972You \\
\hline${ }^{232} \mathrm{Th}$ & 0 & 11.10 & 32 & 3.51 & 137 & 0.477 & 249 & 13.97 & 20 & 4.22 & 72 & 0.671 & 226 & 1.148 & 336 & $10.2-18.3$ & 1976Gu1 \\
\hline & 4 & 11.45 & 17 & 4.30 & 70 & 0.849 & 184 & 14.26 & 6 & 3.77 & 40 & 0.620 & 139 & 1.469 & 231 & $10.4-18.3$ & $1980 \mathrm{Ca} 1$ \\
\hline & 4 & 12.61 & 67 & 7.84 & 152 & 1.429 & 434 & 14.30 & 16 & 1.90 & 153 & 0.114 & 146 & 1.543 & 458 & $9.2-16.3$ & 1973Ve1 \\
\hline & 5 & 11.35 & 24 & 3.97 & 74 & 0.581 & 160 & 14.17 & 14 & 3.98 & 59 & 0.542 & 144 & 1.123 & 215 & $10.0-18.1$ & 2007Var \\
\hline & 10 & 11.23 & 3 & 3.36 & 7 & 0.586 & 25 & 14.12 & 3 & 4.35 & 17 & 0.799 & 37 & 1.385 & 45 & $9.4-17.8$ & $1980 \mathrm{Ca} 1$ \\
\hline${ }^{233} \mathrm{U}$ & 4 & 11.01 & 6 & 2.23 & 17 & 0.220 & 31 & 13.68 & 7 & 5.38 & 16 & 1.022 & 44 & 1.243 & 54 & $9.4-17.8$ & 1986Be2 \\
\hline & 11 & 11.14 & 3 & 2.51 & 7 & 0.472 & 28 & 14.20 & 3 & 5.17 & 9 & 2.172 & 44 & 2.644 & 52 & $9.4-17.8$ & 1986Be2 \\
\hline${ }^{234} \mathrm{U}$ & 4 & 11.30 & 9 & 2.83 & 30 & 0.568 & 80 & 14.23 & 9 & 4.04 & 38 & 0.670 & 88 & 1.238 & 119 & $9.4-17.8$ & 1986Be2 \\
\hline & 11 & 11.18 & 6 & 2.60 & 21 & 0.713 & 74 & 14.39 & 4 & 4.20 & 22 & 1.575 & 92 & 2.288 & 118 & $9.4-17.8$ & 1986Be2 \\
\hline${ }^{235} \mathrm{U}$ & 0 & 11.11 & 29 & 4.52 & 83 & 0.730 & 186 & 13.92 & 15 & 3.88 & 67 & 0.489 & 153 & 1.219 & 240 & $9.5-18.4$ & 1976Gu1 \\
\hline & 4 & 11.07 & 5 & 2.79 & 15 & 0.494 & 35 & 14.24 & 5 & 4.38 & 22 & 0.853 & 50 & 1.347 & 61 & $9.4-18.3$ & $1980 \mathrm{Ca} 1$ \\
\hline${ }^{236} \mathrm{U}$ & 4 & 11.25 & 7 & 3.34 & 20 & 0.530 & 50 & 14.18 & 6 & 4.25 & 24 & 0.696 & 57 & 1.226 & 76 & $9.5-17.8$ & 1980Ca1 \\
\hline & 10 & 11.11 & 4 & 3.20 & 11 & 0.499 & 29 & 14.06 & 5 & 4.34 & 16 & 0.727 & 37 & 1.226 & 47 & $9.4-17.8$ & $1980 \mathrm{Ca} 1$ \\
\hline${ }^{238} \mathrm{U}$ & 0 & 11.24 & 18 & 3.54 & 54 & 0.533 & 104 & 14.45 & 15 & 4.26 & 55 & 0.604 & 111 & 1.137 & 152 & $9.2-18.8$ & 1976Gu1 \\
\hline & 4 & 11.04 & 5 & 3.62 & 13 & 0.667 & 35 & 14.18 & 4 & 4.34 & 18 & 0.747 & 40 & 1.414 & 53 & $9.0-18.3$ & 1980Ca1 \\
\hline & 4 & 11.10 & 5 & 3.20 & 15 & 0.503 & 33 & 14.20 & 5 & 4.15 & 20 & 0.647 & 40 & 1.150 & 52 & $9.1-17.8$ & 1973Ve1 \\
\hline & 5 & 11.10 & 10 & 3.28 & 24 & 0.469 & 53 & 14.19 & 10 & 4.33 & 35 & 0.623 & 64 & 1.093 & 83 & $9.2-18.2$ & 2007Var \\
\hline${ }^{n a t} \mathrm{U}$ & 2 & 10.86 & 2 & 2.83 & 14 & 0.464 & 29 & 13.96 & 3 & 4.69 & 11 & 0.801 & 31 & 1.264 & 42 & $10.2-17.9$ & 1985Ahr \\
\hline
\end{tabular}


Table 2 (continued)

\begin{tabular}{|c|c|c|c|c|c|c|c|c|c|c|c|c|c|c|c|c|c|}
\hline \multirow{2}{*}{$\begin{array}{l}\mathrm{Nucl} \\
{ }^{237} \mathrm{~Np}\end{array}$} & \multirow{2}{*}{$\begin{array}{l}\text { Id } \\
4\end{array}$} & \multicolumn{2}{|l|}{$\begin{array}{c}E_{r, 1} \\
(\mathrm{MeV})\end{array}$} & \multicolumn{2}{|c|}{$\begin{array}{c}\Gamma_{r, 1} \\
(\mathrm{MeV}) \\
\end{array}$} & \multicolumn{2}{|l|}{$s_{1}$} & \multicolumn{2}{|c|}{$\begin{array}{c}E_{r, 2} \\
(\mathrm{MeV}) \\
\end{array}$} & \multicolumn{2}{|c|}{$\begin{array}{c}\Gamma_{r, 2} \\
(\mathrm{MeV})\end{array}$} & \multicolumn{2}{|l|}{$s_{2}$} & \multicolumn{2}{|l|}{$s$} & \multirow{2}{*}{$\begin{array}{c}\begin{array}{c}\varepsilon_{\min }-\varepsilon_{\max } \\
(\mathrm{MeV})\end{array} \\
9.4-17.8\end{array}$} & \multirow{2}{*}{$\begin{array}{c}\text { Ref } \\
1986 \mathrm{Be} 2\end{array}$} \\
\hline & & 11.105 & 5 & 2.72 & 12 & 0.462 & 32 & 14.27 & 5 & 4.27 & 19 & 0.986 & 46 & 1.448 & 56 & & \\
\hline & 4 & 11.20 & 22 & 3.50 & 71 & 0.483 & 136 & 14.24 & 14 & 4.22 & 64 & 0.682 & 149 & 1.165 & 202 & $9.5-16.5$ & 1973Ve1 \\
\hline & 5 & $11.04 \quad 1$ & 13 & 2.87 & 32 & 0.376 & 64 & 14.20 & 13 & 4.30 & 47 & 0.758 & 90 & 1.133 & 110 & $9.3-17.7$ & 2007Var \\
\hline & 10 & $11.09 ?$ & 7 & 2.68 & 16 & 0.452 & 45 & 14.26 & 8 & 4.32 & 30 & 1.000 & 70 & 1.452 & 83 & $9.4-17.8$ & $1986 \mathrm{Be} 2$ \\
\hline & 10 & 11.213 & 37 & 3.59 & 98 & 0.494 & 209 & 14.32 & 26 & 4.46 & 125 & 0.710 & 265 & 1.204 & 337 & $9.2-16.6$ & 1973Ve1 \\
\hline \multirow[t]{3}{*}{${ }^{239} \mathrm{Pu}$} & 0 & $11.47 \quad 4$ & 46 & 4.50 & 108 & 0.626 & 260 & 14.37 & 26 & 4.76 & 98 & 0.609 & 253 & 1.234 & 362 & $9.3-18.7$ & $1976 \mathrm{Gu} 1$ \\
\hline & 5 & $11.76 \quad 4$ & 47 & 4.03 & 108 & 0.759 & 319 & 14.08 & 17 & 2.41 & 164 & 0.252 & 258 & 1.012 & 410 & $9.2-17.7$ & 2007Var \\
\hline & 10 & $11.41 \quad 1$ & 16 & 3.05 & 24 & 0.515 & 111 & 14.05 & 24 & 4.23 & 72 & 0.641 & 150 & 1.156 & 187 & $9.1-17.8$ & 1986Be2 \\
\hline
\end{tabular}


Table 3

References to experimental and evaluated cross section data taken from EXFOR.

\begin{tabular}{|c|c|c|c|c|}
\hline Nucl & Id & Reaction & Ref & EXFOR \\
\hline \multirow[t]{2}{*}{${ }^{6} \mathrm{Li}$} & 2 & $\gamma$, abs & 1986Var & M0140016 \\
\hline & 4 & $\gamma, \mathrm{sn}$ & 1965Be1 & L0008005 \\
\hline \multirow[t]{3}{*}{${ }^{7} \mathrm{Li}$} & 0 & $\gamma$, abs & 1985Ahr & M0188012 \\
\hline & 2 & $\gamma$, abs & 1986Var & M0140024 \\
\hline & 4 & $\gamma, \mathrm{sn}$ & 1973Bra & L0030005 \\
\hline${ }^{9} \mathrm{Be}$ & 0 & $\gamma$, abs & $1975 \mathrm{Ahr}$ & M0825004 \\
\hline${ }^{10} \mathrm{~B}$ & 4 & $\gamma, \mathrm{sn}$ & 1987Ahs & M0207002 \\
\hline \multirow[t]{4}{*}{${ }^{12} \mathrm{C}$} & 0 & $\gamma$, abs & 1969Bez & L0064002 \\
\hline & 1 & $\gamma$, abs & $1975 \mathrm{Ahr}$ & M0372004 \\
\hline & 3 & $\gamma$, abs & 2002Ish & M0648002 \\
\hline & 4 & $\gamma, \mathrm{sn}$ & 1963Bur & M0160002 \\
\hline${ }^{13} \mathrm{C}$ & 3 & $\gamma$, abs & $2002 \mathrm{Ish}$ & M0648003 \\
\hline${ }^{14} \mathrm{C}$ & 3 & $\gamma$, abs & 2002 Ish & M0648004 \\
\hline \multirow{4}{*}{${ }^{\text {nat }} \mathrm{C}$} & 0 & $\gamma$ abs & $1985 \mathrm{Ahr}$ & M0188014 \\
\hline & 0 & $\gamma$, abs & $1975 \mathrm{Ahr}$ & M0825006 \\
\hline & 0 & $\gamma$, abs & 1972Ahr & L0083005 \\
\hline & 1 & $\gamma$, abs & $1965 \mathrm{Wyc}$ & L0122003 \\
\hline \multirow[t]{2}{*}{${ }^{14} \mathrm{~N}$} & 0 & $\gamma$, abs & 1969Bez & L0064003 \\
\hline & 3 & $\gamma$, abs & 2002Ish & M0648005 \\
\hline${ }^{15} \mathrm{~N}$ & 3 & $\gamma$, abs & 2002 Ish & M0648006 \\
\hline \multirow[t]{6}{*}{${ }^{16} \mathrm{O}$} & 0 & $\gamma$, abs & $1975 \mathrm{Ahr}$ & M0372005 \\
\hline & 0 & $\gamma$, abs & $1972 \mathrm{Ahr}$ & L0083004 \\
\hline & 0 & $\gamma$, abs & 1969Bez & L0064004 \\
\hline & 0 & $\gamma$, abs & 1963Bur & L0127002 \\
\hline & 1 & $\gamma$, abs & $1965 \mathrm{Wyc}$ & L0122004 \\
\hline & 3 & $\gamma$, abs & 2002 Ish & M0648007 \\
\hline${ }^{17} \mathrm{O}$ & 3 & $\gamma$, abs & 2002Ish & M0648008 \\
\hline${ }^{18} \mathrm{O}$ & 3 & $\gamma$, abs & 2002Ish & M0648009 \\
\hline \multirow[t]{2}{*}{ nat $O$} & 0 & $\gamma$, abs & $1985 \mathrm{Ahr}$ & M0188015 \\
\hline & 0 & $\gamma$, abs & $1975 \mathrm{Ahr}$ & M0825008 \\
\hline${ }^{19} \mathrm{~F}$ & 3 & $\gamma$, abs & 2002Ish & M0648010 \\
\hline \multirow[t]{4}{*}{${ }^{23} \mathrm{Na}$} & 0 & $\gamma$, abs & 1981Ish & M0043025 \\
\hline & 1 & $\gamma$, abs & $1965 \mathrm{Wyc}$ & L0122005 \\
\hline & 3 & $\gamma$, abs & 2002Ish & M0648011 \\
\hline & 4 & $\gamma, \mathrm{sn}$ & $1971 \mathrm{Alv}$ & L0022008 \\
\hline \multirow[t]{3}{*}{${ }^{24} \mathrm{Mg}$} & 0 & $\gamma$, abs & 1966Dol & M0727003 \\
\hline & 3 & $\gamma$, abs & 2003Var & M0656003 \\
\hline & 3 & $\gamma$, abs & 2002Ish & M0648012 \\
\hline \multirow[t]{2}{*}{${ }^{25} \mathrm{Mg}$} & 3 & $\gamma$, abs & 2002Ish & M0648013 \\
\hline & 4 & $\gamma, \mathrm{sn}$ & 1971Alv & L0022009 \\
\hline \multirow[t]{3}{*}{${ }^{26} \mathrm{Mg}$} & 3 & $\gamma$, abs & 2003Var & M0656004 \\
\hline & 3 & $\gamma$, abs & 2002Ish & M0648014 \\
\hline & 4 & $\gamma, \mathrm{sn}$ & $1971 \mathrm{Fu} 2$ & L0026006 \\
\hline${ }^{\text {nat }} \mathrm{Mg}$ & 1 & $\gamma$, abs & $1965 \mathrm{Wyc}$ & L0122006 \\
\hline \multirow[t]{6}{*}{${ }^{27} \mathrm{Al}$} & 0 & $\gamma$, abs & $1985 \mathrm{Ahr}$ & M0188016 \\
\hline & 0 & $\gamma$, abs & $1975 \mathrm{Ahr}$ & M0825010 \\
\hline & 0 & $\gamma, \mathrm{abs}$ & 1972Ahr & L0083006 \\
\hline & 0 & $\gamma$, abs & $1975 \mathrm{Ahr}$ & M0372006 \\
\hline & 3 & $\gamma$, abs & 2002Ish & M0648015 \\
\hline & 4 & $\gamma, \mathrm{sn}$ & 1966Fu1 & L0010007 \\
\hline \multirow[t]{2}{*}{${ }^{28} \mathrm{Si}$} & 3 & $\gamma$, abs & 2003Var & M0656005 \\
\hline & 3 & $\gamma$, abs & 2002Ish & M0648016 \\
\hline${ }^{29} \mathrm{Si}$ & 3 & $\gamma$, abs & 2002Ish & M0648017 \\
\hline${ }^{30} \mathrm{Si}$ & 3 & $\gamma$, abs & 2002Ish & M0648018 \\
\hline${ }^{n a t} \mathrm{Si}$ & 0 & $\gamma$, abs & $1975 \mathrm{Ahr}$ & M0372007 \\
\hline${ }^{32} \mathrm{~S}$ & 0 & $\gamma$, abs & 1968Dol & M0824002 \\
\hline & 3 & $\gamma$, abs & 2002Ish & M0648019 \\
\hline${ }^{34} \mathrm{~S}$ & 0 & $\gamma$, abs & 1986Ass & M0510006 \\
\hline & 3 & $\gamma$, abs & 2003Var & M0656006 \\
\hline & 3 & $\gamma$, abs & 2002Ish & M0648020 \\
\hline${ }^{\text {nat }} \mathrm{S}$ & 0 & $\gamma, \mathrm{abs}$ & $1965 \mathrm{Wyc}$ & L0122009 \\
\hline${ }^{40} \mathrm{Ar}$ & 3 & $\gamma$, abs & 2002Ish & M0648021 \\
\hline nat $K$ & 13 & $\gamma, \mathrm{xn}$ & 1974Ve1 & L0039036 \\
\hline${ }^{40} \mathrm{Ca}$ & 0 & $\gamma$, abs & 1966Dol & M0727004 \\
\hline & 3 & $\gamma$, abs & 2003Ero & M0653002 \\
\hline${ }^{42} \mathrm{Ca}$ & 3 & $\gamma$, abs & 2003Ero & M0653003 \\
\hline${ }^{44} \mathrm{Ca}$ & 3 & $\gamma$, abs & 2003Ero & M0653004 \\
\hline
\end{tabular}


Table 3 (continued)

\begin{tabular}{|c|c|c|c|c|}
\hline Nucl & Id & Reaction & Ref & EXFOR \\
\hline \multirow[t]{2}{*}{${ }^{48} \mathrm{Ca}$} & 0 & $\gamma$, abs & 1987OKe & M0636010 \\
\hline & 3 & $\gamma$, abs & 2003Ero & M0653005 \\
\hline \multirow{5}{*}{${ }^{\text {nat }} \mathrm{Ca}$} & 0 & $\gamma$, abs & $1975 \mathrm{Ahr}$ & M0372008 \\
\hline & 0 & $\gamma$, abs & $1972 \mathrm{Ahr}$ & L0083003 \\
\hline & 0 & $\gamma$, abs & $1968 \mathrm{Bez}$ & M0840003 \\
\hline & 1 & $\gamma$, abs & $1965 \mathrm{Wyc}$ & L0122010 \\
\hline & 2 & $\gamma$, abs & 1985Ahr & M0188017 \\
\hline${ }^{46} \mathrm{Ti}$ & 3 & $\gamma$, abs & $2002 \mathrm{Ish}$ & M0648026 \\
\hline${ }^{48} \mathrm{Ti}$ & 3 & $\gamma$, abs & 2002Ish & M0648027 \\
\hline \multirow{3}{*}{${ }^{51} \mathrm{~V}$} & 4 & $\gamma, \mathrm{sn}$ & $1962 \mathrm{Fu} 1$ & L0001008 \\
\hline & 3 & $\gamma$, abs & 2003Var & M0656007 \\
\hline & 4 & $\gamma, \mathrm{sn}$ & 1969Gor & M0093005 \\
\hline \multirow[t]{2}{*}{${ }^{52} \mathrm{Cr}$} & 3 & $\gamma$, abs & 2002Ish & M0648028 \\
\hline & 4 & $\gamma, \mathrm{sn}$ & 1969Gor & M0093006 \\
\hline \multirow[t]{3}{*}{${ }^{55} \mathrm{Mn}$} & 4 & $\gamma, \mathrm{sn}$ & $1979 \mathrm{Al} 2$ & L0028011 \\
\hline & 4 & $\gamma, \mathrm{sn}$ & 1974Car & M0835002 \\
\hline & 4 & $\gamma, \mathrm{sn}$ & 1970Is1 & M0726003 \\
\hline${ }^{54} \mathrm{Fe}$ & 0 & $\gamma$, abs & 1978Nor & M0507004 \\
\hline \multirow[t]{3}{*}{${ }^{59} \mathrm{Co}$} & 4 & $\gamma, \mathrm{sn}$ & 1979Al2 & L0028012 \\
\hline & 4 & $\gamma, \mathrm{sn}$ & $1964 \mathrm{Baz}$ & M0106003 \\
\hline & 4 & $\gamma, \mathrm{sn}$ & 1969Gor & M0093007 \\
\hline \multirow{4}{*}{${ }^{58} \mathrm{Ni}$} & 3 & $\gamma$, abs & 2003Var & M0656008 \\
\hline & 3 & $\gamma$, abs & 2002Ish & M0648029 \\
\hline & 4 & $\gamma, \mathrm{sn}$ & $1974 \mathrm{Fu} 3$ & L0034002 \\
\hline & 4 & $\gamma, \mathrm{sn}$ & 1970Gor & M0597002 \\
\hline \multirow[t]{3}{*}{${ }^{60} \mathrm{Ni}$} & 3 & $\gamma$, abs & 2003Var & M0656002 \\
\hline & 4 & $\gamma, \mathrm{sn}$ & $1974 \mathrm{Fu} 3$ & L0034008 \\
\hline & 4 & $\gamma, \mathrm{sn}$ & 1970Gor & M0597003 \\
\hline \multirow[t]{6}{*}{${ }^{63} \mathrm{Cu}$} & 3 & $\gamma$, abs & 2003Var & M0656010 \\
\hline & 3 & $\gamma$, abs & 2002Ish & M0648031 \\
\hline & 4 & $\gamma, \mathrm{sn}$ & 1964Fu1 & L0006012 \\
\hline & 5 & $\gamma, \mathrm{sn}$ & 2016Va1 & M0920002 \\
\hline & 6 & $\gamma, \mathrm{sn}$ & $1968 \mathrm{Su} 1$ & L0013002,L0013003 \\
\hline & 3 & $\gamma$, abs & 2003Var & M0656011 \\
\hline \multirow[t]{3}{*}{${ }^{65} \mathrm{Cu}$} & 3 & $\gamma$, abs & 2002Ish & M0648032 \\
\hline & 4 & $\gamma, \mathrm{sn}$ & 1964Fu1 & L0006013 \\
\hline & 5 & $\gamma, \mathrm{sn}$ & 2016Va1 & M0920005 \\
\hline \multirow[t]{4}{*}{${ }^{\text {nat }} \mathrm{Cu}$} & 1 & $\gamma$, abs & $1965 \mathrm{Wyc}$ & L0122013 \\
\hline & 4 & $\gamma, \mathrm{sn}$ & 1964Ful & L0006011 \\
\hline & 4 & $\gamma, \mathrm{sn}$ & 1970Is2 & M0537005 \\
\hline & 4 & $\gamma, \mathrm{sn}$ & 1968Tom & L0108004 \\
\hline${ }^{64} \mathrm{Zn}$ & 8 & $\gamma, \mathrm{sn}$ & 1976Ca1 & L0043002,L0043003 \\
\hline${ }^{65} \mathrm{Zn}$ & 1 & $\gamma$, abs & 2003Rod & M0652007 \\
\hline \multirow[t]{2}{*}{${ }^{70} \mathrm{Ge}$} & 0 & $\gamma$, abs & $1975 \mathrm{Mcc}$ & M0496004 \\
\hline & 8 & $\gamma, \mathrm{sn}$ & $1976 \mathrm{Ca} 1$ & L0043008,L0043009 \\
\hline${ }^{72} \mathrm{Ge}$ & 0 & $\gamma$, abs & $1975 \mathrm{Mcc}$ & M0496010 \\
\hline & 8 & $\gamma, \mathrm{sn}$ & $1976 \mathrm{Ca} 1$ & L0043011,L0043012 \\
\hline${ }^{74} \mathrm{Ge}$ & 8 & $\gamma, \mathrm{sn}$ & 1976Ca1 & L0043014,L0043015 \\
\hline & 0 & $\gamma$, abs & $1975 \mathrm{Mcc}$ & M0496013 \\
\hline${ }^{76} \mathrm{Ge}$ & 8 & $\gamma, \mathrm{sn}$ & $1976 \mathrm{Ca} 1$ & L0043017,L0043018 \\
\hline & 0 & $\gamma$, abs & $1975 \mathrm{Mcc}$ & M0496007 \\
\hline${ }^{75} \mathrm{As}$ & 4 & $\gamma, \mathrm{sn}$ & 1969Be1 & L0014012 \\
\hline & 8 & $\gamma, \mathrm{sn}$ & 1976Ca1 & L0043020,L0043021 \\
\hline${ }^{76} \mathrm{Se}$ & 0 & $\gamma$, abs & 1978Gur & M0023002 \\
\hline & 8 & $\gamma, \mathrm{sn}$ & $1976 \mathrm{Ca} 1$ & L0043023,L0043024 \\
\hline${ }^{78} \mathrm{Se}$ & 8 & $\gamma$, sn & $1976 \mathrm{Ca} 1$ & L0043026,L0043027 \\
\hline${ }^{80} \mathrm{Se}$ & 5 & $\gamma, \mathrm{sn}$ & 2016Va1 & M0920008 \\
\hline & 8 & $\gamma, \mathrm{sn}$ & 1976Ca1 & L0043029,L0043030 \\
\hline${ }^{82} \mathrm{Se}$ & 0 & $\gamma$, abs & 1978Gur & M0023003 \\
\hline & 8 & $\gamma, \mathrm{sn}$ & 1976Ca1 & L0043032,L0043033 \\
\hline${ }^{\text {nat }} \mathrm{Rb}$ & 4 & $\gamma$, sn & 1971Lep & L0027017 \\
\hline${ }^{\text {nat }} \mathrm{Sr}$ & 4 & $\gamma$, sn & 1971Lep & L0027018 \\
\hline${ }^{89} \mathrm{Y}$ & 4 & $\gamma, \mathrm{sn}$ & 1971Le1 & L0027019 \\
\hline & 3 & $\gamma$, abs & 2003Var & M0656012 \\
\hline & 4 & $\gamma, \mathrm{sn}$ & 1999Bel & M0706002 \\
\hline & 4 & $\gamma, \mathrm{sn}$ & 1967Be2 & L0011018 \\
\hline & 12 & $\gamma, 1 \mathrm{n}$ & 1972 You & L0059002 \\
\hline${ }^{90} \mathrm{Zr}$ & 3 & $\gamma$, abs & 2003Var & M0656013 \\
\hline
\end{tabular}


Table 3 (continued)

\begin{tabular}{|c|c|c|c|c|}
\hline Nucl & Id & Reaction & Ref & EXFOR \\
\hline & 4 & $\gamma, \mathrm{sn}$ & 1972Ask & M0360003 \\
\hline & 4 & $\gamma, \mathrm{sn}$ & $1967 \mathrm{Be} 2$ & L0011019 \\
\hline & 8 & $\gamma, \mathrm{sn}$ & 1971Le1 & L0027012,L0027013 \\
\hline \multirow[t]{2}{*}{${ }^{91} \mathrm{Zr}$} & 4 & $\gamma, \mathrm{sn}$ & 1967Be2 & L0011020 \\
\hline & 5 & $\gamma, \mathrm{sn}$ & 2015Var & M0914002 \\
\hline${ }^{92} \mathrm{Zr}$ & 4 & $\gamma, \mathrm{sn}$ & $1967 \mathrm{Be} 2$ & L0011021 \\
\hline \multirow[t]{2}{*}{${ }^{94} \mathrm{Zr}$} & 4 & $\gamma, \mathrm{sn}$ & $1967 \mathrm{Be} 2$ & L0011022 \\
\hline & 5 & $\gamma, \mathrm{sn}$ & 2015Var & M0914005 \\
\hline${ }^{93} \mathrm{Nb}$ & 8 & $\gamma, \mathrm{sn}$ & 1971Le1 & L0027015,L0027016 \\
\hline \multirow[t]{3}{*}{${ }^{92} \mathrm{Mo}$} & 3 & $\gamma$, abs & 2003Var & M0656014 \\
\hline & 4 & $\gamma, \mathrm{sn}$ & 1974Be3 & L0032020 \\
\hline & 4 & $\gamma, \mathrm{sn}$ & $1970 \mathrm{Is} 3$ & M0719003 \\
\hline${ }^{94} \mathrm{Mo}$ & 4 & $\gamma, \mathrm{sn}$ & 1974Be3 & L0032021 \\
\hline${ }^{96} \mathrm{Mo}$ & 4 & $\gamma, \mathrm{sn}$ & $1974 \mathrm{Be} 3$ & L0032022 \\
\hline \multirow[t]{2}{*}{${ }^{98} \mathrm{Mo}$} & 4 & $\gamma, \mathrm{sn}$ & $1974 \mathrm{Be} 3$ & L0032023 \\
\hline & 4 & $\gamma, \mathrm{sn}$ & $1970 \mathrm{Is} 3$ & M0719006 \\
\hline${ }^{100} \mathrm{Mo}$ & 9 & $\gamma, \mathrm{sn}$ & 1974Be3 & L0032017,L0032018,L0032019 \\
\hline \multirow[t]{3}{*}{${ }^{103} \mathrm{Rh}$} & 3 & $\gamma$, abs & 2003Var & M0656015 \\
\hline & 4 & $\gamma, \mathrm{sn}$ & 1974Le1 & L0035041 \\
\hline & 4 & $\gamma, \mathrm{sn}$ & 1962Bog & M0769006 \\
\hline${ }^{108} \mathrm{Pd}$ & 0 & $\gamma$, abs & 1969Dea & M0839004 \\
\hline${ }^{\text {nat }} \mathrm{Pd}$ & 4 & $\gamma, \mathrm{sn}$ & 1971Lep & L0035042 \\
\hline \multirow[t]{3}{*}{${ }^{107} \mathrm{Ag}$} & 4 & $\gamma, \mathrm{sn}$ & 1969Ish & M0524002 \\
\hline & 4 & $\gamma, \mathrm{sn}$ & 1969Be1 & L0014013 \\
\hline & 4 & $\gamma, \mathrm{sn}$ & 1962Bog & M0769011 \\
\hline${ }^{109} \mathrm{Ag}$ & 4 & $\gamma, \mathrm{sn}$ & 1969Ish & M0524003 \\
\hline${ }^{\text {nat }} \mathrm{Ag}$ & 4 & $\gamma, \mathrm{sn}$ & 1971Lep & L0035043 \\
\hline${ }^{\text {nat }} \mathrm{Cd}$ & 4 & $\gamma, \mathrm{sn}$ & 1971Lep & L0035044 \\
\hline \multirow[t]{4}{*}{${ }^{115} \mathrm{In}$} & 4 & $\gamma, \mathrm{sn}$ & 1974Le1 & L0035045 \\
\hline & 4 & $\gamma, \mathrm{sn}$ & $1969 \mathrm{Fu} 1$ & L0017029 \\
\hline & 4 & $\gamma, \mathrm{sn}$ & 1962Bog & M0769003 \\
\hline & 5 & $\gamma, \mathrm{sn}$ & 2013Var & M0863005 \\
\hline \multirow[t]{2}{*}{${ }^{112} \mathrm{Sn}$} & 4 & $\gamma, \mathrm{sn}$ & 1974Sor & M0745005 \\
\hline & 5 & $\gamma, \mathrm{sn}$ & 2009Var & M0768038 \\
\hline \multirow[t]{2}{*}{${ }^{114} \mathrm{Sn}$} & 4 & $\gamma, \mathrm{sn}$ & 1975Sor & M0746008 \\
\hline & 5 & $\gamma, \mathrm{sn}$ & 2009Var & M0768039 \\
\hline \multirow[t]{4}{*}{${ }^{116} \mathrm{Sn}$} & 4 & $\gamma, \mathrm{sn}$ & 1974Le1 & L0035046 \\
\hline & 4 & $\gamma, \mathrm{sn}$ & $1969 \mathrm{Fu} 1$ & L0017030 \\
\hline & 4 & $\gamma, \mathrm{sn}$ & 1975Sor & M0746009 \\
\hline & 5 & $\gamma, \mathrm{sn}$ & 2009Var & M0768040 \\
\hline \multirow[t]{4}{*}{${ }^{117} \mathrm{Sn}$} & 4 & $\gamma, \mathrm{sn}$ & 1974Le1 & L0035047 \\
\hline & 4 & $\gamma, \mathrm{sn}$ & $1969 \mathrm{Fu} 1$ & L0017031 \\
\hline & 4 & $\gamma, \mathrm{sn}$ & 1975Sor & M0746010 \\
\hline & 5 & $\gamma, \mathrm{sn}$ & 2009Var & M0768041 \\
\hline \multirow[t]{4}{*}{${ }^{118} \mathrm{Sn}$} & 4 & $\gamma, \mathrm{sn}$ & 1974Le1 & L0035048 \\
\hline & 4 & $\gamma, \mathrm{sn}$ & $1969 \mathrm{Fu} 1$ & L0017032 \\
\hline & 4 & $\gamma, \mathrm{sn}$ & 1974Sor & M0745006 \\
\hline & 5 & $\gamma, \mathrm{sn}$ & 2009Var & M0768042 \\
\hline${ }^{119} \mathrm{Sn}$ & 4 & $\gamma, \mathrm{sn}$ & $1969 \mathrm{Fu} 1$ & L0017033 \\
\hline & 4 & $\gamma, \mathrm{sn}$ & 1975Sor & M0746011 \\
\hline & 5 & $\gamma, \mathrm{sn}$ & 2009Var & M0768043 \\
\hline${ }^{120} \mathrm{Sn}$ & 4 & $\gamma, \mathrm{sn}$ & 1974Le1 & L0035049 \\
\hline & 4 & $\gamma, \mathrm{sn}$ & $1969 \mathrm{Fu} 1$ & L0017034 \\
\hline & 4 & $\gamma, \mathrm{sn}$ & 1974Sor & M0745007 \\
\hline & 5 & $\gamma, \mathrm{sn}$ & 2009Var & M0768044 \\
\hline${ }^{122} \mathrm{Sn}$ & 4 & $\gamma, \mathrm{sn}$ & 1975Sor & M0746012 \\
\hline & 5 & $\gamma, \mathrm{sn}$ & 2009Var & M0768045 \\
\hline${ }^{124} \mathrm{Sn}$ & 3 & $\gamma$, abs & 2003Var & M0656016 \\
\hline & 4 & $\gamma, \mathrm{sn}$ & 1974Le1 & L0035050 \\
\hline & 4 & $\gamma, \mathrm{sn}$ & $1969 \mathrm{Fu} 1$ & L0017035 \\
\hline & 4 & $\gamma, \mathrm{sn}$ & 1975Sor & M0746013 \\
\hline & 5 & $\gamma, \mathrm{sn}$ & 2009Var & M0768046 \\
\hline${ }^{\text {nat }} \mathrm{Sb}$ & 4 & $\gamma, \mathrm{sn}$ & 1971Lep & L0035051 \\
\hline${ }^{124} \mathrm{Te}$ & 6 & $\gamma, \mathrm{sn}$ & 1976Le2 & L0042004,L0042002 \\
\hline${ }^{126} \mathrm{Te}$ & 6 & $\gamma, \mathrm{sn}$ & 1976Le2 & L0042007,L0042005 \\
\hline${ }^{128} \mathrm{Te}$ & 6 & $\gamma, \mathrm{sn}$ & 1976Le2 & L0042010,L0042008 \\
\hline${ }^{130} \mathrm{Te}$ & 6 & $\gamma, \mathrm{sn}$ & 1976Le2 & L0042013,L0042011 \\
\hline${ }^{127} \mathrm{I}$ & 4 & $\gamma, \mathrm{sn}$ & 1999Bel & M0706004 \\
\hline
\end{tabular}


Table 3 (continued)

\begin{tabular}{|c|c|c|c|c|}
\hline Nucl & Id & Reaction & Ref & EXFOR \\
\hline & 4 & $\gamma, \mathrm{sn}$ & 1989Ras & M0511002 \\
\hline & 4 & $\gamma, \mathrm{sn}$ & 1969Be6 & L0015022 \\
\hline & 4 & $\gamma, \mathrm{sn}$ & $1966 \mathrm{Br} 1$ & L0009009 \\
\hline & 5 & $\gamma, \mathrm{sn}$ & 2006Var & M0693003 \\
\hline & 8 & $\gamma, \mathrm{sn}$ & 1987Ber & L0057005,L0057006 \\
\hline \multirow{3}{*}{${ }^{133} \mathrm{Cs}$} & 4 & $\gamma, \mathrm{sn}$ & 1974Le1 & L0035053 \\
\hline & 4 & $\gamma, \mathrm{sn}$ & 1969Be1 & L0014014 \\
\hline & 5 & $\gamma, \mathrm{sn}$ & $2016 \mathrm{Va} 2$ & M0922002 \\
\hline \multirow[t]{2}{*}{${ }^{138} \mathrm{Ba}$} & 5 & $\gamma, \mathrm{sn}$ & 2016Va2 & M0922006 \\
\hline & 4 & $\gamma, \mathrm{sn}$ & $1970 \mathrm{Be} 8$ & L0019008 \\
\hline${ }^{\text {nat }} \mathrm{Ba}$ & 4 & $\gamma, \mathrm{sn}$ & 1971Be4 & L0024015 \\
\hline \multirow[t]{4}{*}{${ }^{139} \mathrm{La}$} & 4 & $\gamma, \mathrm{sn}$ & 1972De1 & M0398004 \\
\hline & 4 & $\gamma, \mathrm{sn}$ & 1971Be4 & L0024016 \\
\hline & 4 & $\gamma, \mathrm{sn}$ & 1968Be5 & L0012018 \\
\hline & 4 & $\gamma, \mathrm{sn}$ & 1964Ric & M0928003 \\
\hline${ }^{140} \mathrm{Ce}$ & 6 & $\gamma, \mathrm{sn}$ & 1976Le2 & L0042016,L0042014 \\
\hline${ }^{142} \mathrm{Ce}$ & 6 & $\gamma, \mathrm{sn}$ & 1976Le2 & L0042019,L0042017 \\
\hline \multirow[t]{7}{*}{${ }^{141} \mathrm{Pr}$} & 4 & $\gamma, \mathrm{xn}$ & 1987Ber & L0057015 \\
\hline & 4 & $\gamma, \mathrm{sn}$ & $1966 \mathrm{Br} 1$ & L0009010 \\
\hline & 4 & $\gamma, \mathrm{sn}$ & 1964Ric & M0928005 \\
\hline & 12 & $\gamma, 1 \mathrm{n}$ & 1972You & L0059003 \\
\hline & 12 & $\gamma, 1 \mathrm{n}$ & 1971Be4 & L0024011 \\
\hline & 12 & $\gamma, 1 \mathrm{n}$ & 1970Su1 & L0020002 \\
\hline & 13 & $\gamma, \mathrm{xn}$ & 1972De1 & M0398002 \\
\hline \multirow[t]{2}{*}{${ }^{142} \mathrm{Nd}$} & 3 & $\gamma$, abs & 2003Var & M0656017 \\
\hline & 4 & $\gamma, \mathrm{sn}$ & $1971 \mathrm{Ca} 1$ & L0025023 \\
\hline${ }^{143} \mathrm{Nd}$ & 4 & $\gamma, \mathrm{sn}$ & $1971 \mathrm{Ca} 1$ & L0025024 \\
\hline${ }^{144} \mathrm{Nd}$ & 4 & $\gamma, \mathrm{sn}$ & 1971Ca1 & L0025025 \\
\hline${ }^{145} \mathrm{Nd}$ & 4 & $\gamma, \mathrm{sn}$ & 1971Ca1 & L0025026 \\
\hline \multirow[t]{2}{*}{${ }^{146} \mathrm{Nd}$} & 4 & $\gamma, \mathrm{sn}$ & $1971 \mathrm{Ca} 1$ & L0025027 \\
\hline & 4 & $\gamma, \mathrm{sn}$ & 1971Vas & M0817003 \\
\hline \multirow[t]{2}{*}{${ }^{148} \mathrm{Nd}$} & 4 & $\gamma, \mathrm{sn}$ & 1971Ca1 & L0025028 \\
\hline & 4 & $\gamma, \mathrm{sn}$ & 1969Vas & M0816003 \\
\hline${ }^{150} \mathrm{Nd}$ & 4 & $\gamma$, sn & $1971 \mathrm{Ca} 1$ & L0025029 \\
\hline${ }^{144} \mathrm{Sm}$ & 4 & $\gamma, \mathrm{sn}$ & $1974 \mathrm{Ca} 5$ & L0033017 \\
\hline \multirow[t]{2}{*}{${ }^{148} \mathrm{Sm}$} & 4 & $\gamma, \mathrm{sn}$ & $1974 \mathrm{Ca} 5$ & L0033018 \\
\hline & 4 & $\gamma, \mathrm{sn}$ & 1971Vas & M0817005 \\
\hline \multirow[t]{2}{*}{${ }^{150} \mathrm{Sm}$} & 4 & $\gamma, \mathrm{sn}$ & $1974 \mathrm{Ca} 5$ & L0033019 \\
\hline & 4 & $\gamma, \mathrm{sn}$ & 1969Vas & M0816007 \\
\hline \multirow[t]{2}{*}{${ }^{152} \mathrm{Sm}$} & 4 & $\gamma, \mathrm{sn}$ & $1974 \mathrm{Ca} 5$ & L0033020 \\
\hline & 4 & $\gamma, \mathrm{sn}$ & 1969Vas & M0816009 \\
\hline \multirow[t]{3}{*}{${ }^{154} \mathrm{Sm}$} & 0 & $\gamma$, abs & $1981 \mathrm{Gur}$ & M0073002 \\
\hline & 4 & $\gamma, \mathrm{sn}$ & $1974 \mathrm{Ca} 5$ & L0033021 \\
\hline & 4 & $\gamma, \mathrm{sn}$ & 1969Vas & M0816011 \\
\hline${ }^{151} \mathrm{Eu}$ & 4 & $\gamma, \mathrm{sn}$ & 1971 Vas & M0817015 \\
\hline \multirow[t]{2}{*}{${ }^{153} \mathrm{Eu}$} & 4 & $\gamma, \mathrm{sn}$ & 1969Be8 & L0016018 \\
\hline & 4 & $\gamma, \mathrm{sn}$ & 1971Vas & M0817017 \\
\hline${ }^{152} \mathrm{Gd}$ & 4 & $\gamma, \mathrm{sn}$ & 1971Vas & M0817007 \\
\hline${ }^{154} \mathrm{Gd}$ & 4 & $\gamma, \mathrm{sn}$ & 1971Vas & M0817009 \\
\hline${ }^{156} \mathrm{Gd}$ & 0 & $\gamma$, abs & $1981 \mathrm{Gur}$ & M0073003 \\
\hline & 4 & $\gamma, \mathrm{sn}$ & 1971Vas & M0817011 \\
\hline${ }^{158} \mathrm{Gd}$ & 4 & $\gamma, \mathrm{sn}$ & 1971Vas & M0817013 \\
\hline${ }^{160} \mathrm{Gd}$ & 3 & $\gamma$, abs & 2003Var & M0656018 \\
\hline & 4 & $\gamma, \mathrm{sn}$ & 1969Be8 & L0016019 \\
\hline${ }^{159} \mathrm{~Tb}$ & 4 & $\gamma, \mathrm{sn}$ & 1976Gor & M0057002 \\
\hline & 4 & $\gamma, \mathrm{sn}$ & 1968Be5 & L0012019 \\
\hline & 4 & $\gamma, \mathrm{sn}$ & 1964Br1 & L0005006 \\
\hline & 4 & $\gamma, \mathrm{sn}$ & 1962Bog & M0769014 \\
\hline & 4 & $\gamma, \mathrm{sn}$ & $1958 \mathrm{Ful}$ & L0111002 \\
\hline & 5 & $\gamma, \mathrm{sn}$ & 2011Var & M0831005 \\
\hline${ }^{165} \mathrm{Ho}$ & 0 & $\gamma$, abs & $1981 \mathrm{Gur}$ & M0073004 \\
\hline & 0 & $\gamma$, abs & $1976 \mathrm{Gu} 2$ & M0056002 \\
\hline & 4 & $\gamma, \mathrm{sn}$ & 1969Be8 & L0016020 \\
\hline & 4 & $\gamma, \mathrm{sn}$ & 1968Be5 & L0012020 \\
\hline & 4 & $\gamma, \mathrm{sn}$ & 1966Axe & M0844003 \\
\hline & 4 & $\gamma, \mathrm{sn}$ & 1976Gor & M0057006 \\
\hline${ }^{168} \mathrm{Er}$ & 0 & $\gamma$, abs & 1981Gur & M0073005 \\
\hline${ }^{174} \mathrm{Yb}$ & 0 & $\gamma$, abs & $1981 \mathrm{Gur}$ & M0073006 \\
\hline
\end{tabular}


Table 3 (continued)

\begin{tabular}{|c|c|c|c|c|}
\hline Nucl & $\mathrm{Id}$ & Reaction & Ref & EXFOR \\
\hline${ }^{175} \mathrm{Lu}$ & 4 & $\gamma, \mathrm{sn}$ & 1969Be6 & L0015026 \\
\hline${ }^{176} \mathrm{Hf}$ & 4 & $\gamma, \mathrm{sn}$ & 1977Gor & M0007002 \\
\hline \multirow[t]{4}{*}{${ }^{178} \mathrm{Hf}$} & 0 & $\gamma$, abs & $1981 \mathrm{Gur}$ & M0073007 \\
\hline & 0 & $\gamma$, abs & $1976 \mathrm{Gu} 2$ & M0056003 \\
\hline & 4 & $\gamma, \mathrm{sn}$ & 1977Gor & M0007003 \\
\hline & 4 & $\gamma, \mathrm{sn}$ & 1976Gor & M0057012 \\
\hline \multirow[t]{3}{*}{${ }^{180} \mathrm{Hf}$} & 0 & $\gamma$, abs & $1981 \mathrm{Gur}$ & M0073008 \\
\hline & 0 & $\gamma$, abs & $1976 \mathrm{Gu} 2$ & M0056004 \\
\hline & 4 & $\gamma, \mathrm{sn}$ & 1977Gor & M0007004 \\
\hline \multirow[t]{11}{*}{${ }^{181} \mathrm{Ta}$} & 0 & $\gamma$, abs & $1981 \mathrm{Gur}$ & M0073009 \\
\hline & 0 & $\gamma$, abs & 1976Gu2 & M0056005 \\
\hline & 3 & $\gamma$, abs & 2003Var & M0656019 \\
\hline & 4 & $\gamma, \mathrm{sn}$ & 2001Bel & M0707003 \\
\hline & 4 & $\gamma, \mathrm{sn}$ & 1968Be5 & L0012021 \\
\hline & 4 & $\gamma, \mathrm{sn}$ & $1963 \mathrm{Br} 1$ & L0003005 \\
\hline & 4 & $\gamma$, sn & $1962 \mathrm{Bog}$ & M0769009 \\
\hline & 4 & $\gamma, \mathrm{sn}$ & 1969Ish & M0737003 \\
\hline & 4 & $\gamma, \mathrm{sn}$ & 1967Ant & M0539005 \\
\hline & 4 & $\gamma, \mathrm{sn}$ & $1958 \mathrm{Ful}$ & L0111003 \\
\hline & 5 & $\gamma, \mathrm{sn}$ & 2012Var & M0850006 \\
\hline \multirow[t]{3}{*}{${ }^{182} \mathrm{~W}$} & 0 & $\gamma$, abs & $1981 \mathrm{Gur}$ & M0073010 \\
\hline & 0 & $\gamma$, abs & 1976Gu2 & M0056006 \\
\hline & 4 & $\gamma, \mathrm{sn}$ & $1978 \mathrm{Go} 3$ & M0025002 \\
\hline \multirow[t]{3}{*}{${ }^{184} \mathrm{~W}$} & 0 & $\gamma$, abs & $1981 \mathrm{Gur}$ & M0073011 \\
\hline & 4 & $\gamma, \mathrm{sn}$ & 1978Go3 & M0025003 \\
\hline & 4 & $\gamma, \mathrm{sn}$ & 1973Gor & M0645007 \\
\hline \multirow[t]{4}{*}{${ }^{186} \mathrm{~W}$} & 0 & $\gamma$, abs & $1981 \mathrm{Gur}$ & M0073012 \\
\hline & 4 & $\gamma, \mathrm{sn}$ & 1978Go3 & M0025004 \\
\hline & 4 & $\gamma, \mathrm{sn}$ & 1969Be8 & L0016021 \\
\hline & 4 & $\gamma$, sn & 1973Gor & M0645008 \\
\hline${ }^{185} \mathrm{Re}$ & 4 & $\gamma, \mathrm{sn}$ & 1973Gor & M0645009 \\
\hline${ }^{\text {nat }} \operatorname{Re}$ & 4 & $\gamma, \mathrm{sn}$ & 1975 Vey & M0845013 \\
\hline \multirow[t]{2}{*}{${ }^{186} \mathrm{Os}$} & 5 & $\gamma, \mathrm{sn}$ & 2015Var & M0915002 \\
\hline & 7 & $\gamma, \mathrm{sn}$ & 1979Be4 & L0046004,L0046002 \\
\hline \multirow[t]{2}{*}{${ }^{188} \mathrm{Os}$} & 5 & $\gamma$, sn & 2014Var & M0916005 \\
\hline & 9 & $\gamma$, sn & 1979Be4 & L0046005,L0046006,L0046007 \\
\hline \multirow[t]{2}{*}{${ }^{189} \mathrm{Os}$} & 5 & $\gamma, \mathrm{sn}$ & 2014Var & M0916009 \\
\hline & 9 & $\gamma$, sn & 1979Be4 & L0046009,L0046010,L0046011 \\
\hline \multirow[t]{2}{*}{${ }^{190} \mathrm{Os}$} & 5 & $\gamma, \mathrm{sn}$ & 2015Var & M0915007 \\
\hline & 9 & $\gamma, \mathrm{sn}$ & 1979Be4 & L0046013,L0046014,L0046015 \\
\hline \multirow[t]{2}{*}{${ }^{192} \mathrm{Os}$} & 5 & $\gamma, \mathrm{sn}$ & 2015Var & M0915011 \\
\hline & 9 & $\gamma, \mathrm{sn}$ & 1979Be4 & L0046017,L0046018,L0046019 \\
\hline \multirow[t]{2}{*}{${ }^{191} \mathrm{Ir}$} & 4 & $\gamma, \mathrm{sn}$ & 1978Go1 & M0008002 \\
\hline & 4 & $\gamma, \mathrm{sn}$ & 1978Go2 & M0049002 \\
\hline${ }^{193} \mathrm{Ir}$ & 4 & $\gamma, \mathrm{sn}$ & $1978 \mathrm{Go} 1$ & M0008003 \\
\hline${ }^{\text {nat }}$ Ir & 4 & $\gamma, \mathrm{sn}$ & $1975 \mathrm{Vey}$ & M0845014 \\
\hline${ }^{194} \mathrm{Pt}$ & 4 & $\gamma, \mathrm{sn}$ & 1978Go1 & M0008004 \\
\hline & 13 & $\gamma, \mathrm{xn}$ & $1978 \mathrm{Go} 2$ & M0049006 \\
\hline${ }^{195} \mathrm{Pt}$ & 4 & $\gamma, \mathrm{sn}$ & 1978Go1 & M0008005 \\
\hline & 13 & $\gamma, \mathrm{xn}$ & 1978Go2 & M0049008 \\
\hline${ }^{196} \mathrm{Pt}$ & 4 & $\gamma, \mathrm{sn}$ & 1978Go1 & M0008006 \\
\hline${ }^{198} \mathrm{Pt}$ & 4 & $\gamma, \mathrm{sn}$ & 1978Go1 & M0008007 \\
\hline${ }^{197} \mathrm{Au}$ & 0 & $\gamma$, abs & $1981 \mathrm{Gur}$ & M0073013 \\
\hline & 0 & $\gamma$, abs & 1976Gu2 & M0056007 \\
\hline & 4 & $\gamma, \mathrm{sn}$ & 1970Ve1 & L0021010 \\
\hline & 4 & $\gamma$, sn & $1962 \mathrm{Fu} 2$ & L0002005 \\
\hline & 4 & $\gamma, \mathrm{sn}$ & 1973Sor & M0650003 \\
\hline & 4 & $\gamma, \mathrm{sn}$ & $1958 \mathrm{Ful}$ & L0111004 \\
\hline & 8 & $\gamma, \mathrm{sn}$ & 1987Ber & L0057009,L0057010 \\
\hline & 8 & $\gamma, \mathrm{sn}$ & 2010Var & M0798003 \\
\hline${ }^{203} \mathrm{Tl}$ & 4 & $\gamma, \mathrm{sn}$ & 1970Ant & L0109002 \\
\hline${ }^{205} \mathrm{Tl}$ & 4 & $\gamma, \mathrm{sn}$ & 1970Ant & L0109003 \\
\hline${ }^{206} \mathrm{~Pb}$ & 4 & $\gamma, \mathrm{sn}$ & $1964 \mathrm{Ha} 2$ & L0007014 \\
\hline & 4 & $\gamma, \mathrm{sn}$ & 1973Sor & L0107002 \\
\hline${ }^{207} \mathrm{~Pb}$ & 4 & $\gamma, \mathrm{sn}$ & $1964 \mathrm{Ha} 2$ & L0007015 \\
\hline${ }^{208} \mathrm{~Pb}$ & 3 & $\gamma$, abs & 2003Var & M0656020 \\
\hline & 4 & $\gamma, \mathrm{sn}$ & 1995Bel & M0702003 \\
\hline & 4 & $\gamma, \mathrm{sn}$ & 1970Ve1 & L0021011 \\
\hline
\end{tabular}


Table 3 (continued)

\begin{tabular}{|c|c|c|c|c|}
\hline Nucl & Id & Reaction & Ref & EXFOR \\
\hline & 4 & $\gamma, \mathrm{sn}$ & 1964На2 & L0007016 \\
\hline & 4 & $\gamma, \mathrm{sn}$ & 1973Sor & L0107004 \\
\hline & 5 & $\gamma, \mathrm{sn}$ & 2013Ish & M0864028 \\
\hline & 12 & $\gamma, 1 \mathrm{n}$ & 1972You & L0059004 \\
\hline \multirow[t]{2}{*}{${ }^{\text {nat }} \mathrm{Pb}$} & 2 & $\gamma$, abs & $1985 \mathrm{Ahr}$ & M0188018 \\
\hline & 8 & $\gamma, \mathrm{sn}$ & 1987Ber & L0057012,L0057013 \\
\hline \multirow[t]{7}{*}{${ }^{209} \mathrm{Bi}$} & 0 & $\gamma$, abs & $1976 \mathrm{Gu} 2$ & M0056008 \\
\hline & 4 & $\gamma, \mathrm{sn}$ & 1995Bel & M0702004 \\
\hline & 4 & $\gamma, \mathrm{sn}$ & 1964Ha2 & L0007017 \\
\hline & 4 & $\gamma, \mathrm{sn}$ & 1973Sor & M0650005 \\
\hline & 4 & $\gamma, \mathrm{sn}$ & 1967Ant & M0539008 \\
\hline & 5 & $\gamma, \mathrm{sn}$ & 2016Va2 & M0922010 \\
\hline & 12 & $\gamma, \mathrm{sn}$ & 1972 You & L0059005 \\
\hline \multirow[t]{5}{*}{${ }^{232} \mathrm{Th}$} & 0 & $\gamma$, abs & 1976Gu1 & M0090002 \\
\hline & 4 & $\gamma, \mathrm{sn}$ & $1980 \mathrm{Ca} 1$ & L0050030 \\
\hline & 4 & $\gamma, \mathrm{sn}$ & 1973Ve1 & L0031014 \\
\hline & 5 & $\gamma, \mathrm{sn}$ & 2007Var & M0722003 \\
\hline & 10 & $\gamma, \mathrm{sn}$ & 1980Ca1 & L0050002,L0050003,L0050004 \\
\hline \multirow[t]{2}{*}{${ }^{233} \mathrm{U}$} & 4 & $\gamma, \mathrm{sn}$ & $1986 \mathrm{Be} 2$ & L0058026 \\
\hline & 11 & $\gamma, \mathrm{sn}$ & $1986 \mathrm{Be} 2$ & L0058004,L0058003,L0058002 \\
\hline \multirow[t]{2}{*}{${ }^{234} \mathrm{U}$} & 4 & $\gamma$, sn & $1986 \mathrm{Be} 2$ & L0058027 \\
\hline & 11 & $\gamma, \mathrm{sn}$ & $1986 \mathrm{Be} 2$ & L0058007,L0058006,L0058005 \\
\hline \multirow[t]{2}{*}{${ }^{235} \mathrm{U}$} & 0 & $\gamma$, abs & 1976Gu1 & M0090003 \\
\hline & 4 & $\gamma, \mathrm{sn}$ & $1980 \mathrm{Ca} 1$ & L0050032 \\
\hline \multirow[t]{2}{*}{${ }^{236} \mathrm{U}$} & 4 & $\gamma, \mathrm{sn}$ & $1980 \mathrm{Ca} 1$ & L0050033 \\
\hline & 10 & $\gamma, \mathrm{sn}$ & $1980 \mathrm{Ca} 1$ & L0050010,L0050011,L0050012 \\
\hline \multirow[t]{4}{*}{${ }^{238} \mathrm{U}$} & 0 & $\gamma$, abs & 1976Gu1 & M0090004 \\
\hline & 4 & $\gamma, \mathrm{sn}$ & $1980 \mathrm{Ca} 1$ & L0050031 \\
\hline & 4 & $\gamma, \mathrm{sn}$ & $1973 \mathrm{Ve} 1$ & L0031015 \\
\hline & 5 & $\gamma, \mathrm{sn}$ & 2007Var & M0722008 \\
\hline${ }^{n a t} \mathrm{U}$ & 2 & $\gamma$, abs & $1985 \mathrm{Ahr}$ & M0188019 \\
\hline \multirow[t]{5}{*}{${ }^{237} \mathrm{~Np}$} & 4 & $\gamma$, sn & $1986 \mathrm{Be} 2$ & L0058033 \\
\hline & 4 & $\gamma, \mathrm{sn}$ & 1973Ve1 & L0031017 \\
\hline & 5 & $\gamma, \mathrm{sn}$ & 2007Var & M0722013 \\
\hline & 10 & $\gamma, \mathrm{sn}$ & $1986 \mathrm{Be} 2$ & L0058008,L0058009,L0058010 \\
\hline & 10 & $\gamma, \mathrm{sn}$ & 1973Ve1 & L0031007,L0031008,L0031009g \\
\hline \multirow[t]{3}{*}{${ }^{239} \mathrm{Pu}$} & 0 & $\gamma$, abs & $1976 \mathrm{Gu} 1$ & M0090005 \\
\hline & 5 & $\gamma, \mathrm{sn}$ & 2007Var & M0722018 \\
\hline & 10 & $\gamma, \mathrm{sn}$ & $1986 \mathrm{Be} 2$ & L0058012,L0058013,L0058014 \\
\hline
\end{tabular}


Graphs 1 - 162

${ }^{6} \mathrm{Li}(\gamma, \mathrm{abs})$

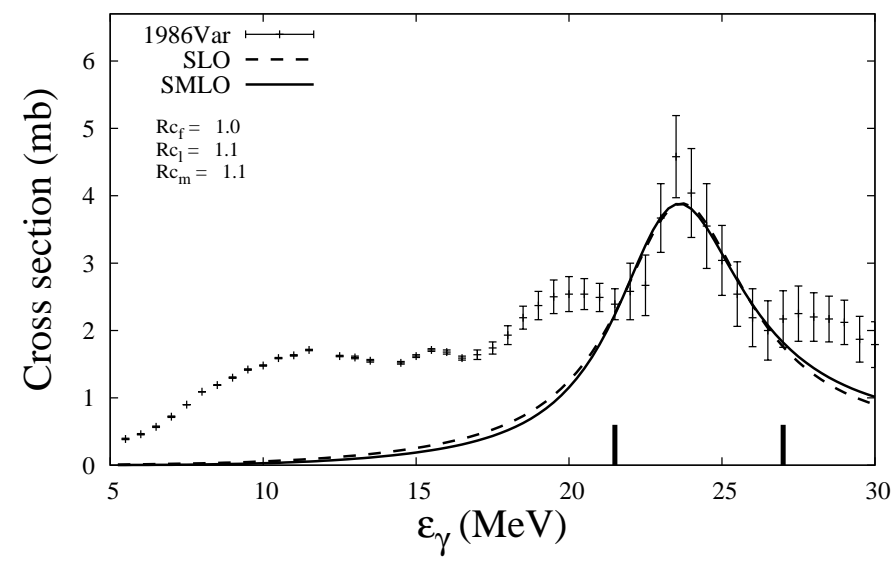

${ }^{9} \mathrm{Be}(\gamma, \mathrm{abs})$

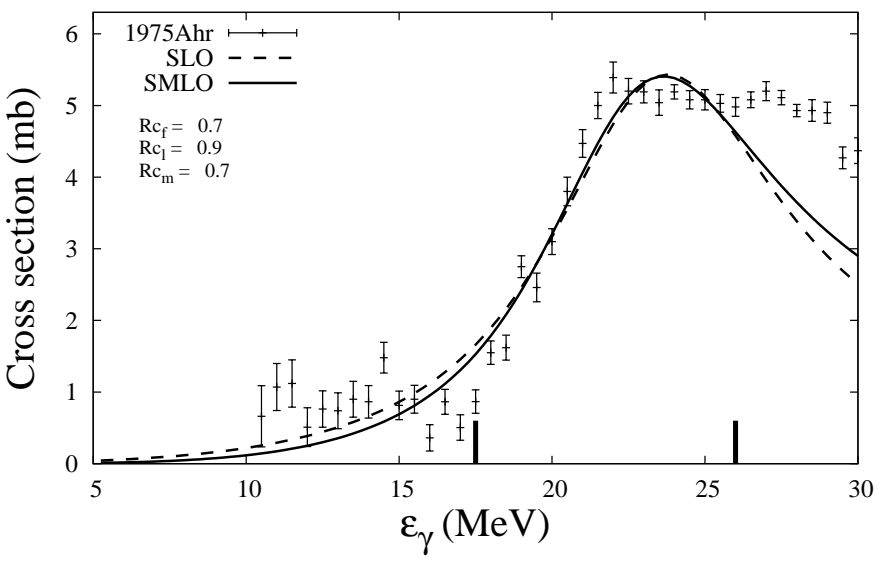

${ }^{\text {nat }} \mathrm{C}(\gamma, \mathrm{abs})$

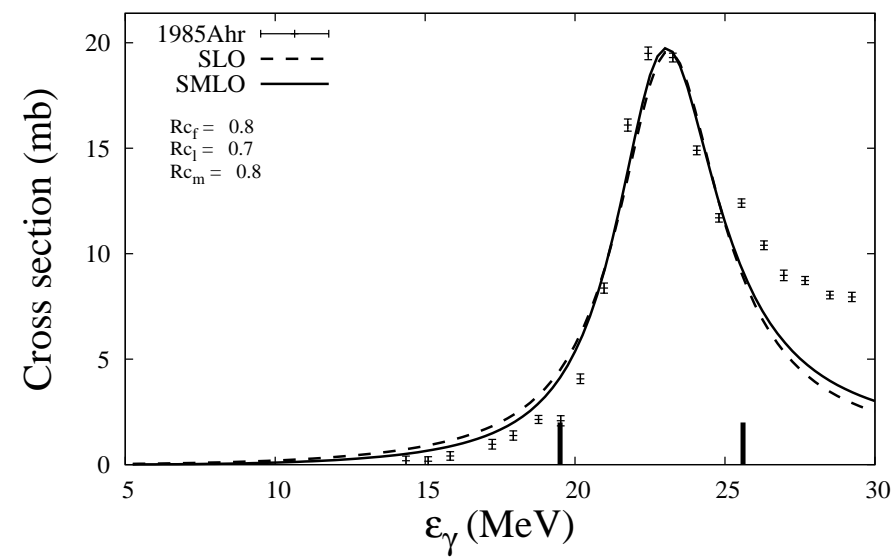

${ }^{7} \operatorname{Li}(\gamma, \mathrm{abs})$

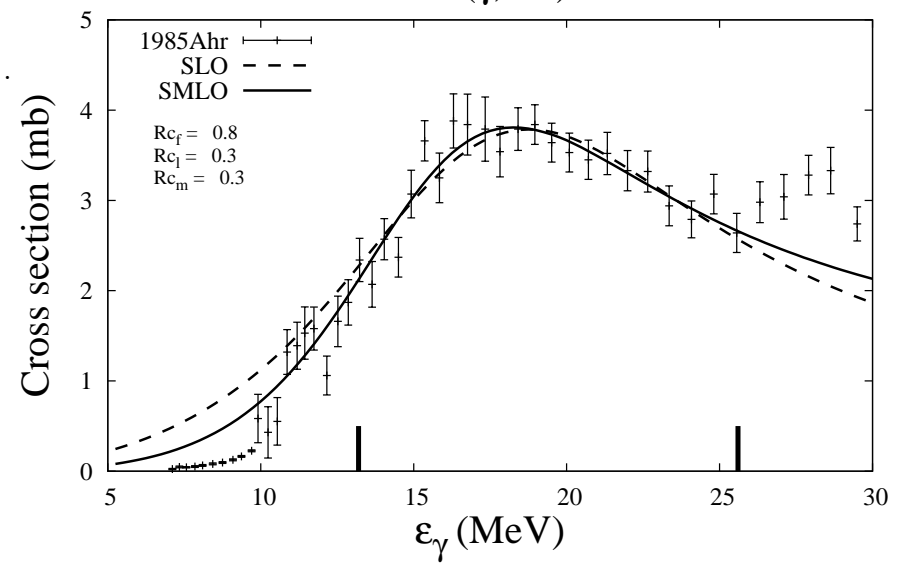

${ }^{10} \mathrm{~B}(\gamma, \mathrm{sn})$

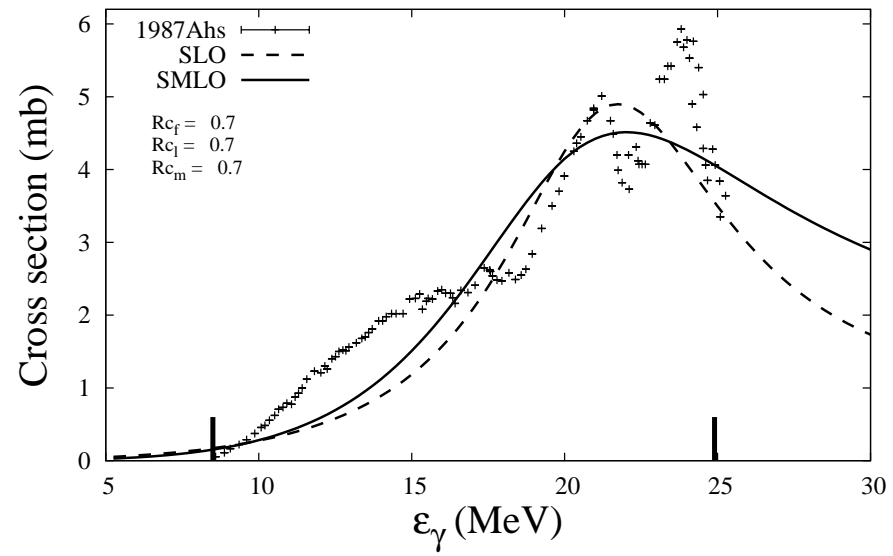

${ }^{12} \mathrm{C}(\gamma, \mathrm{abs})$

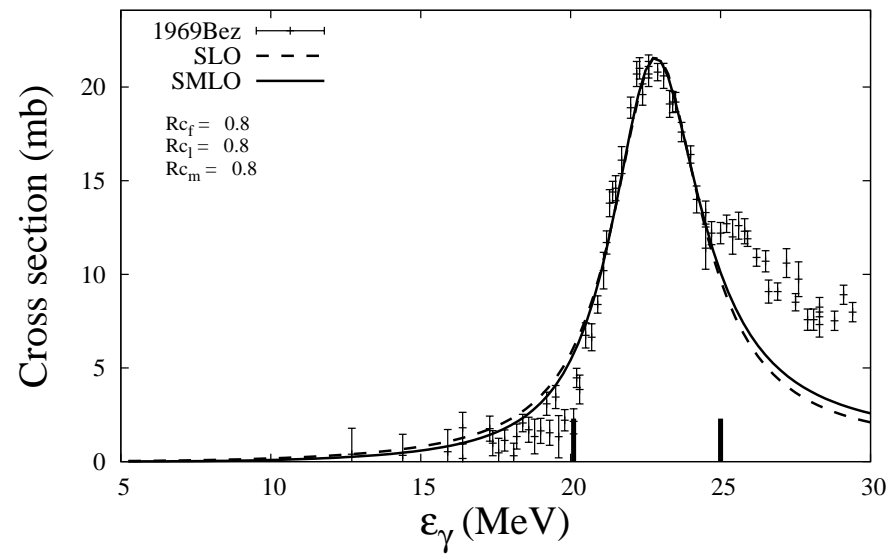



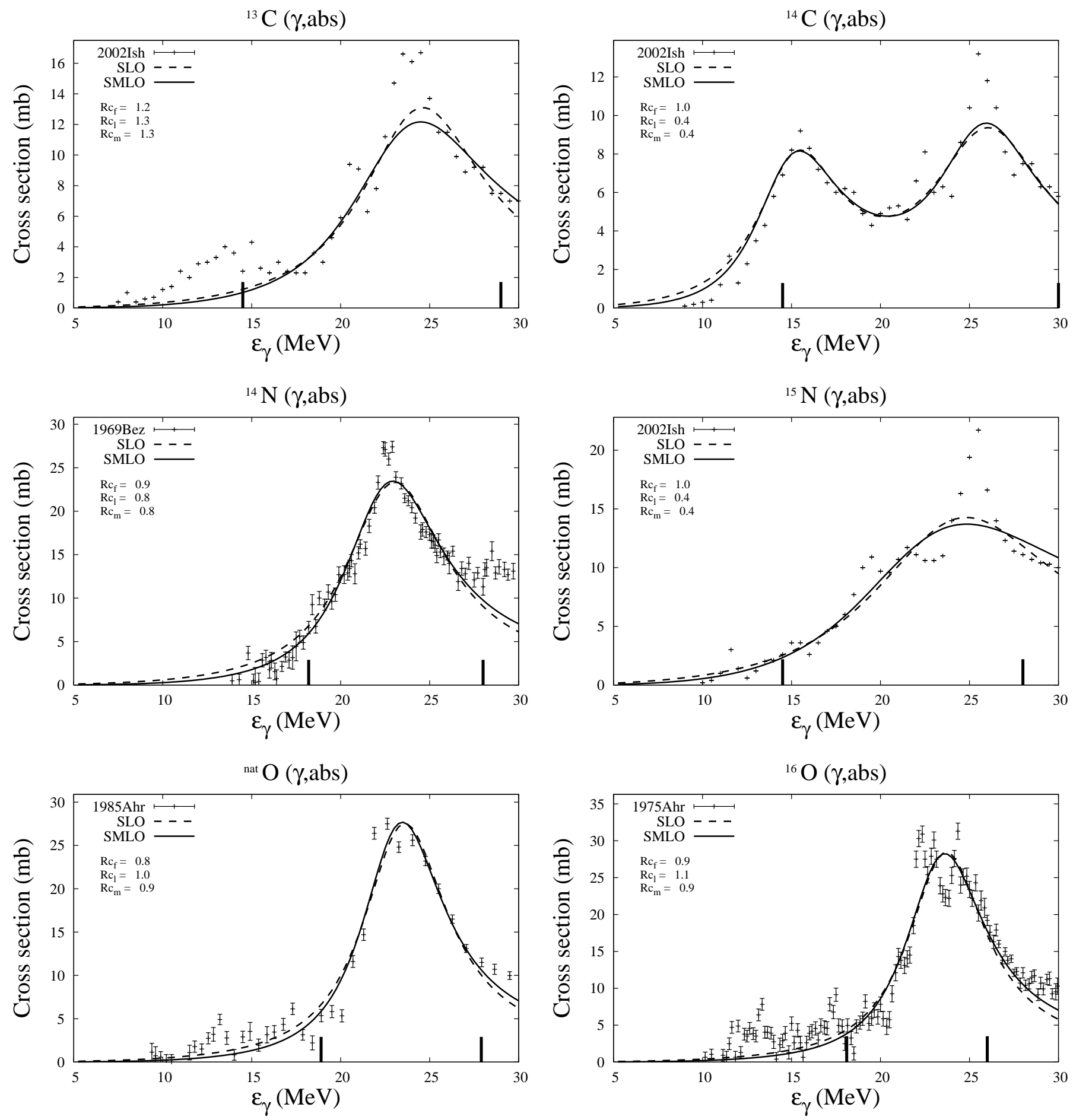

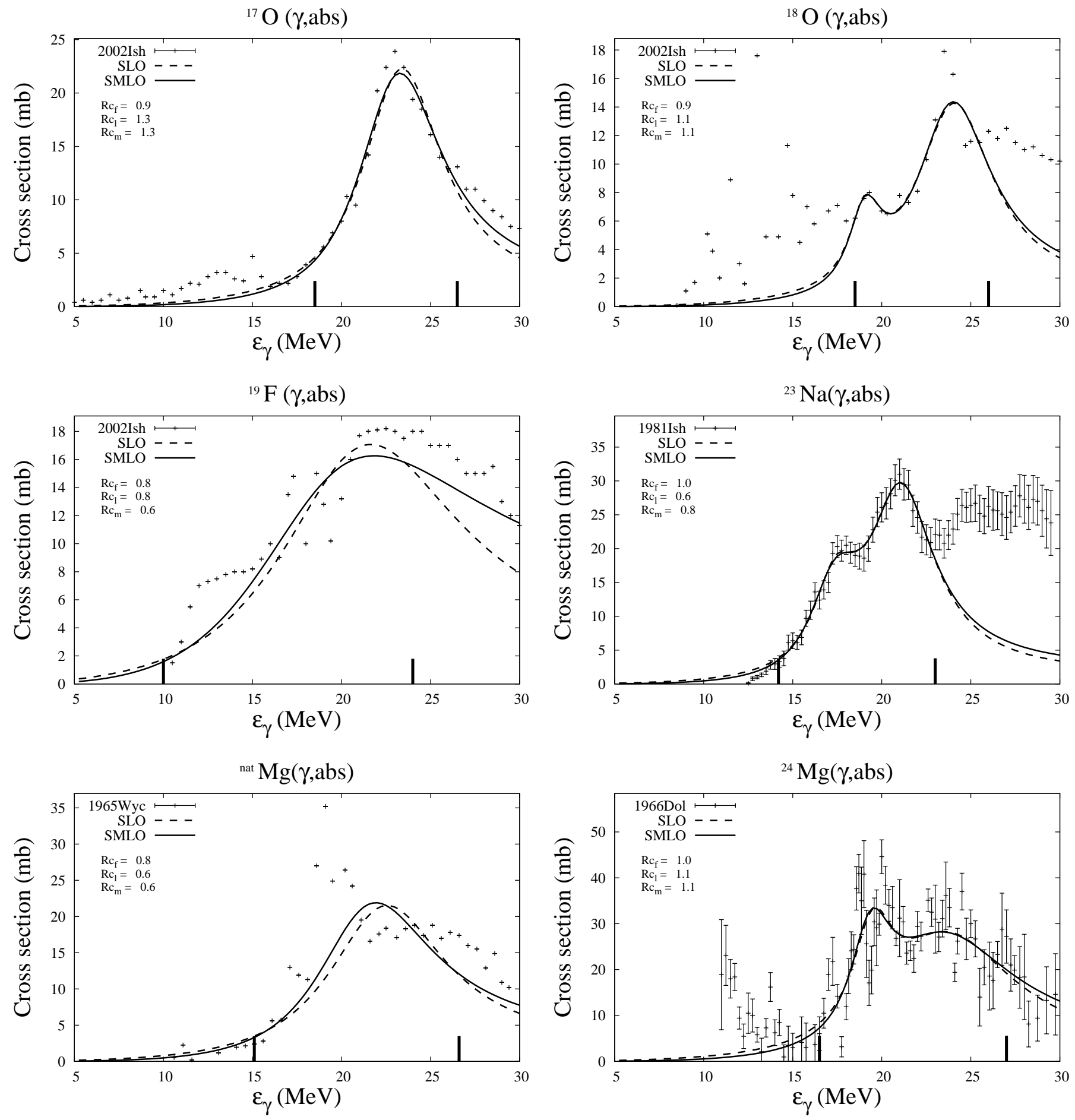

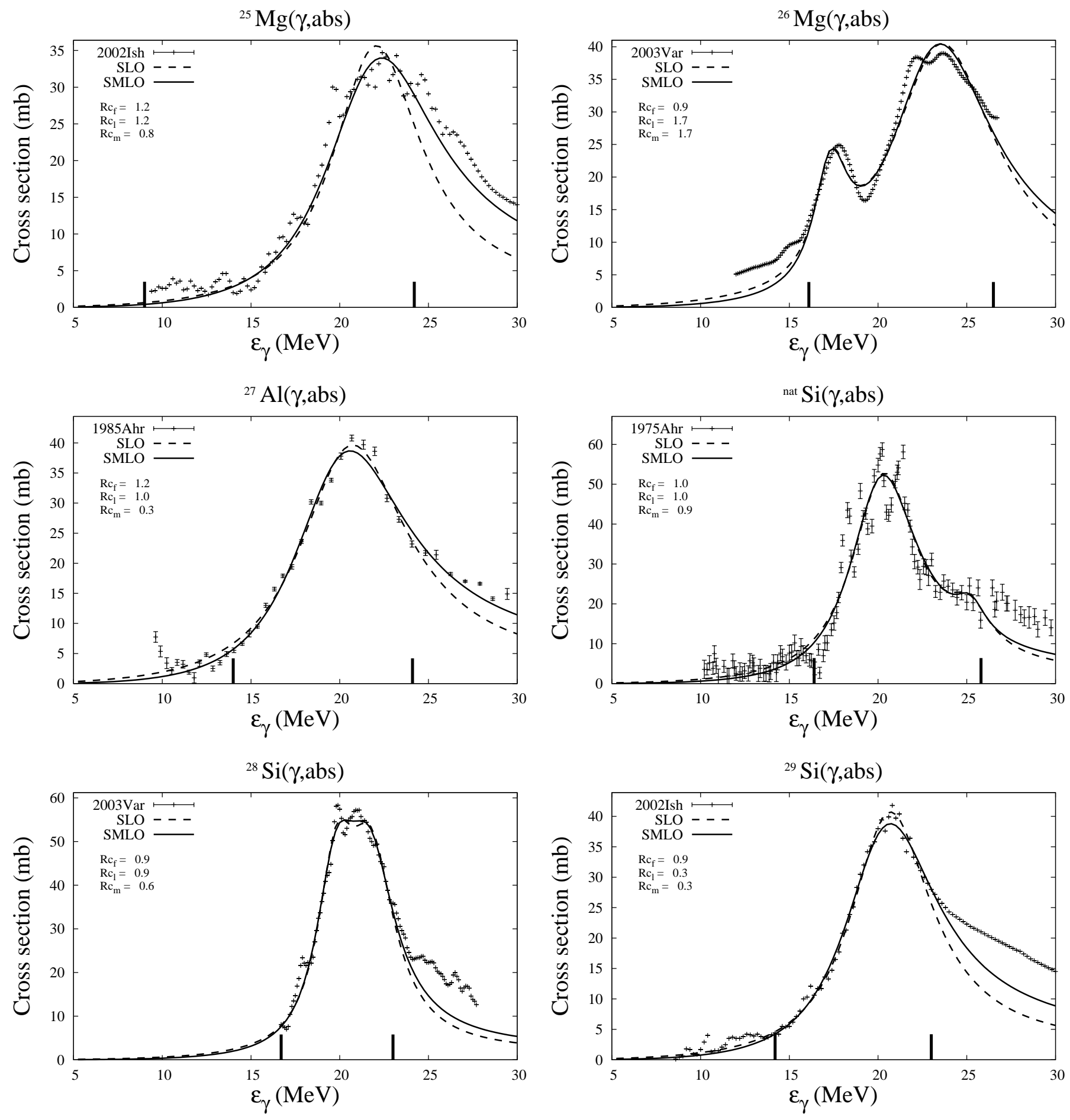

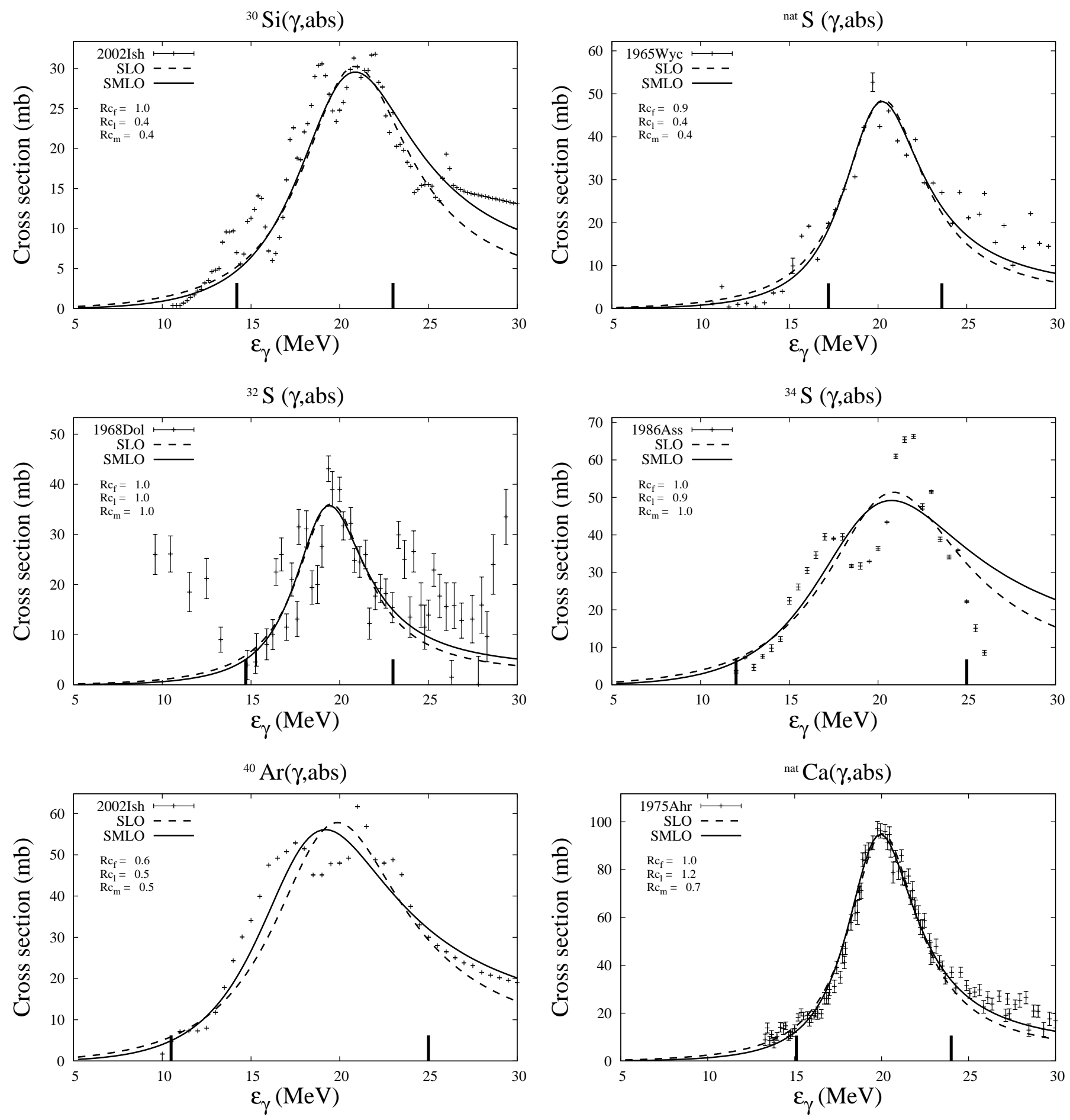

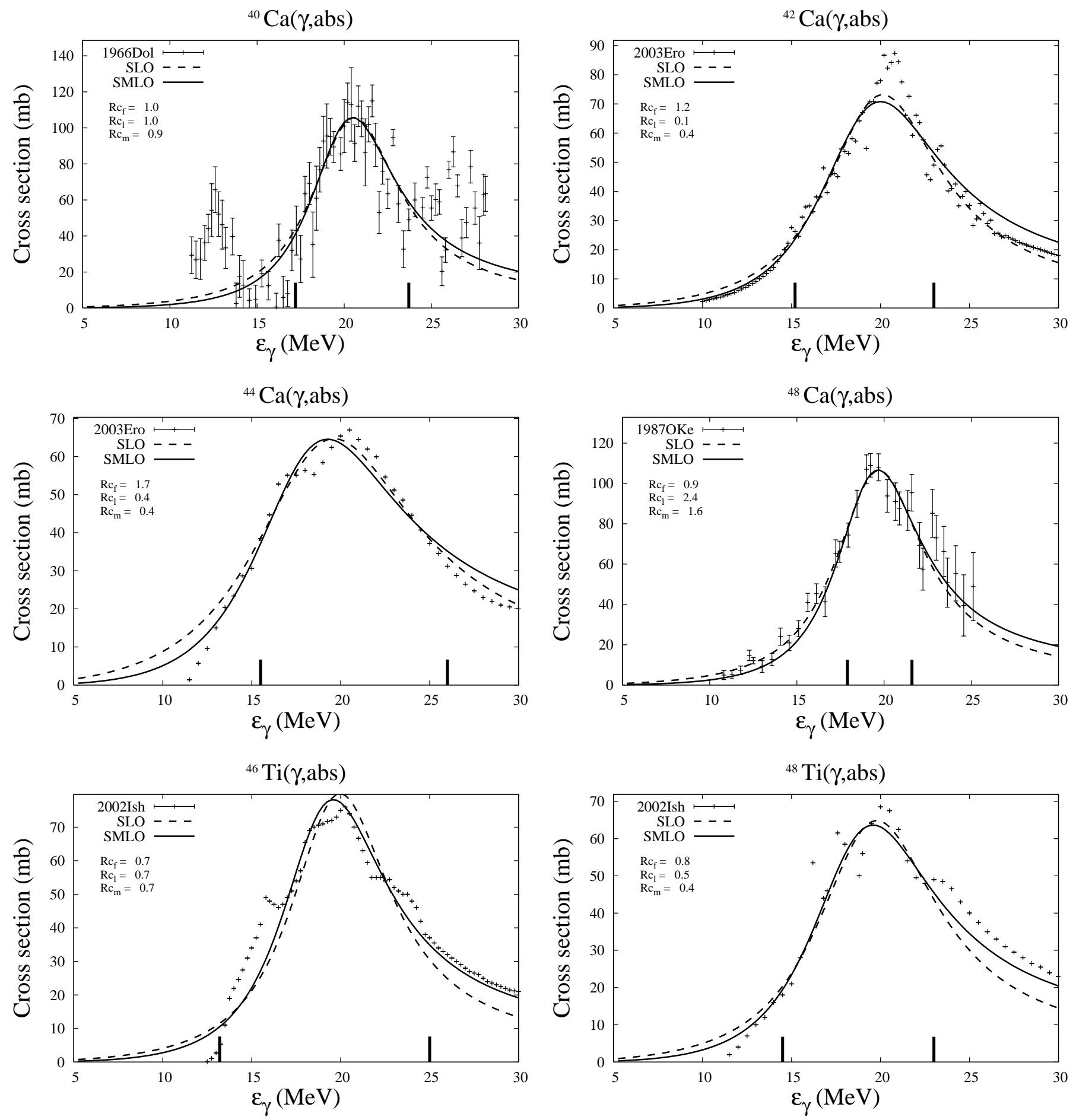

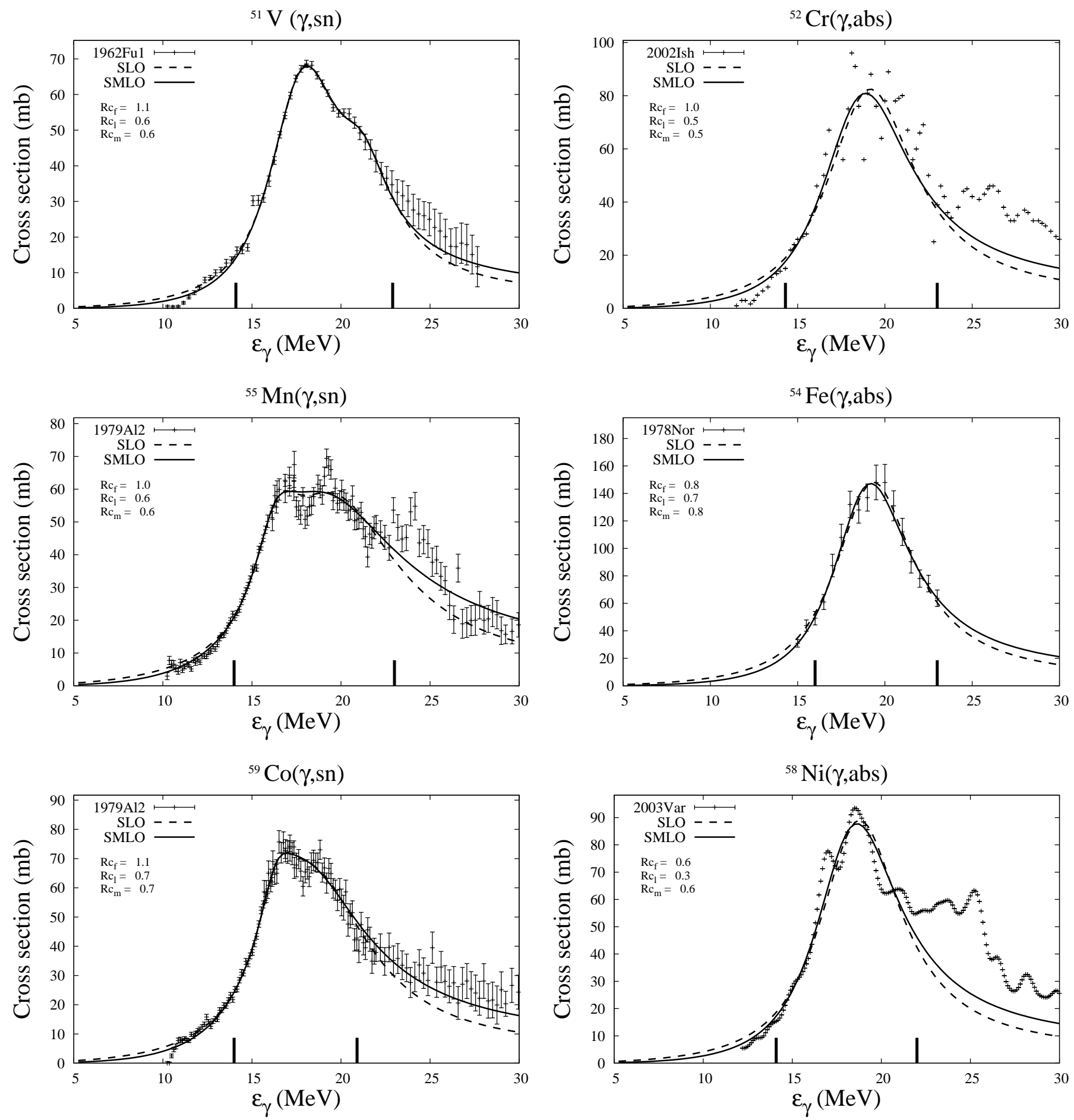

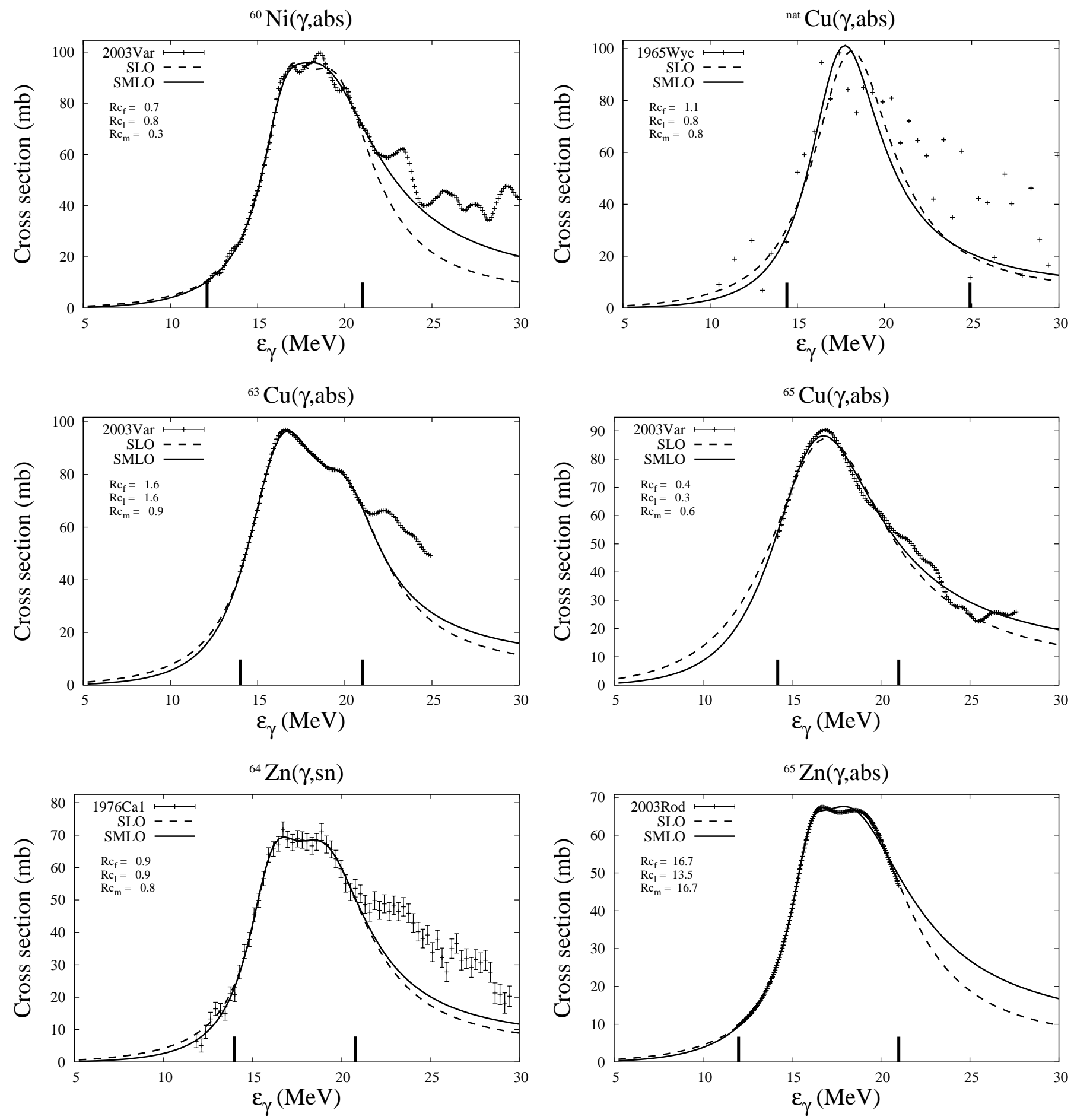

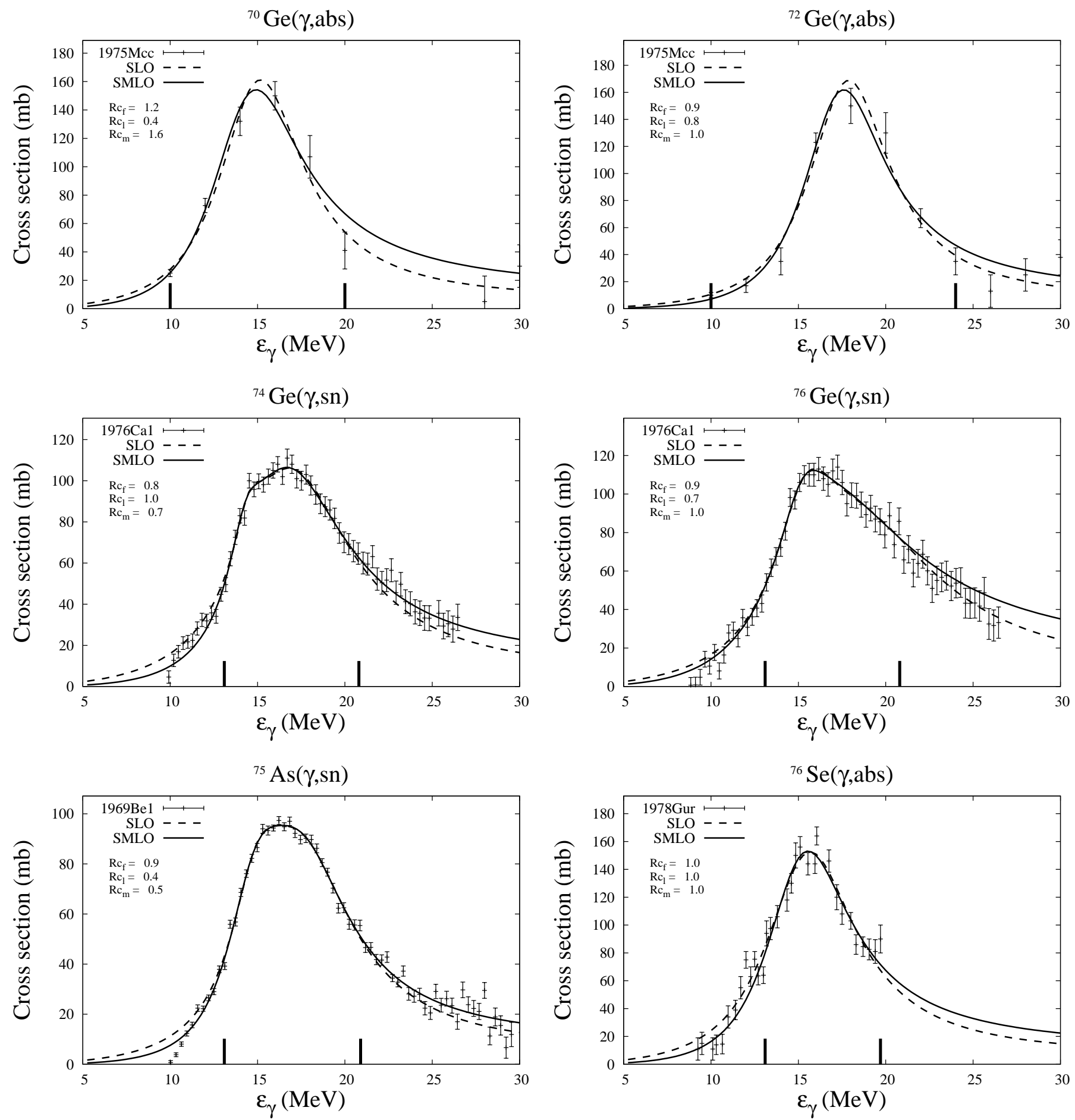

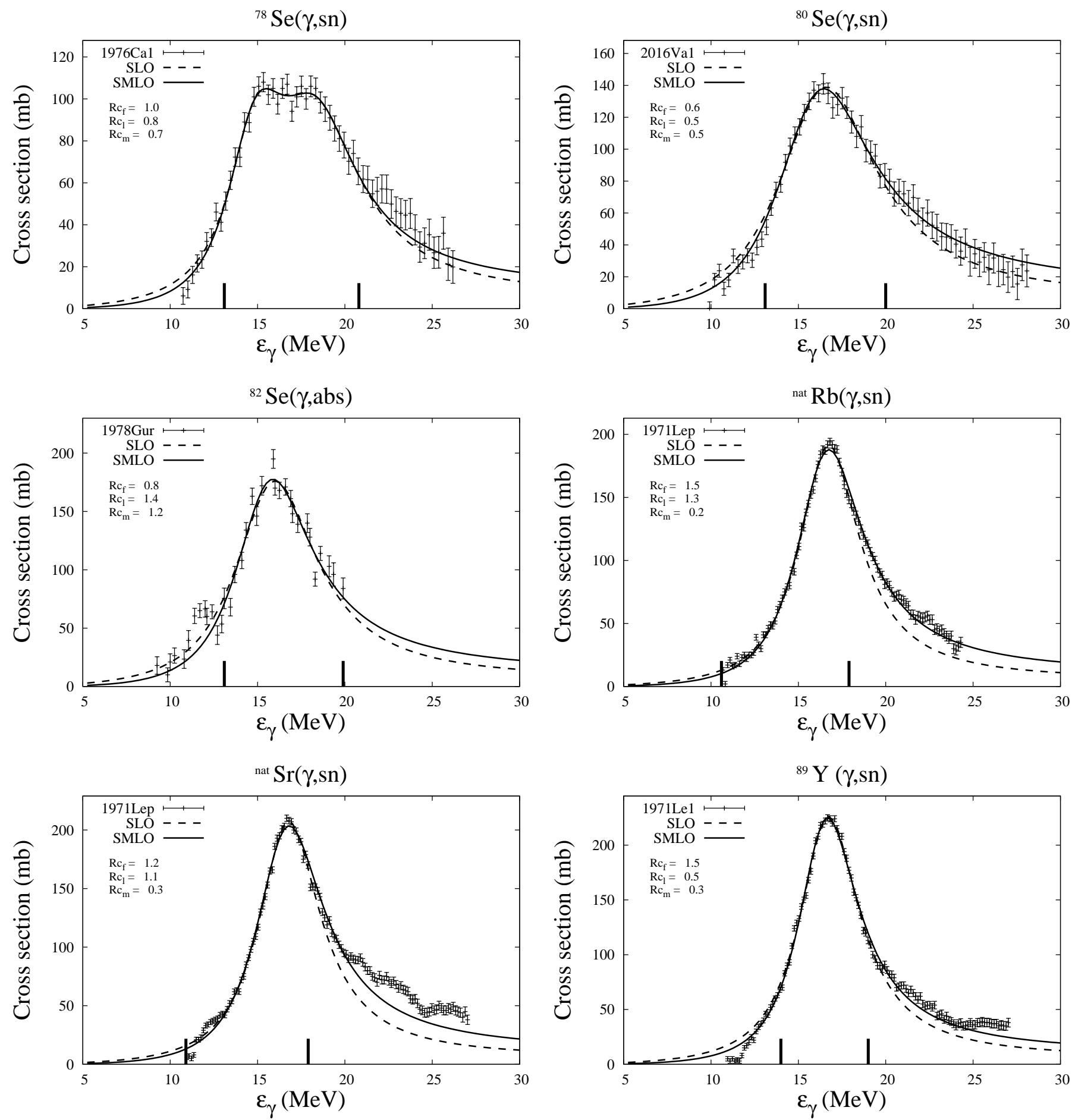

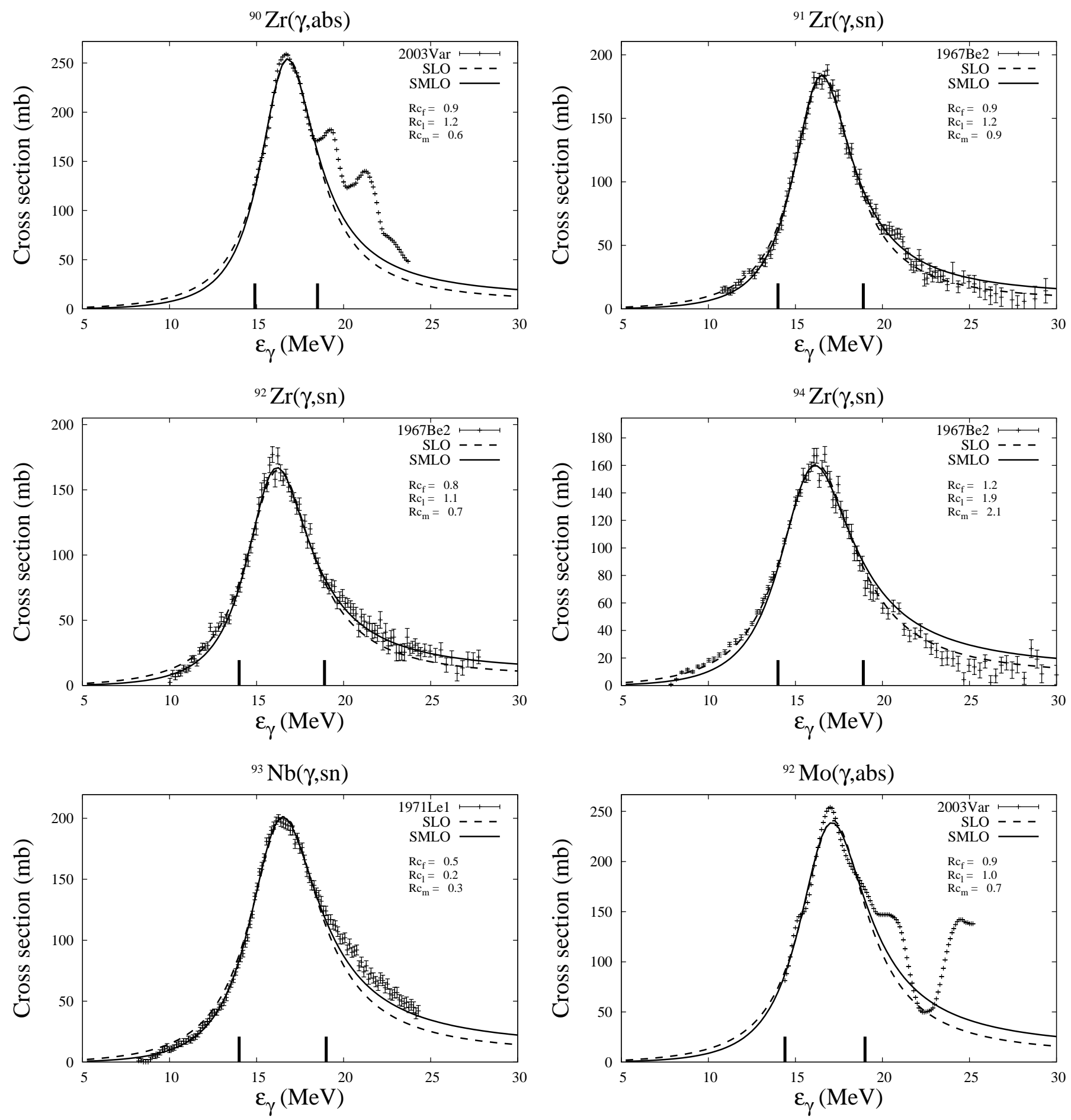

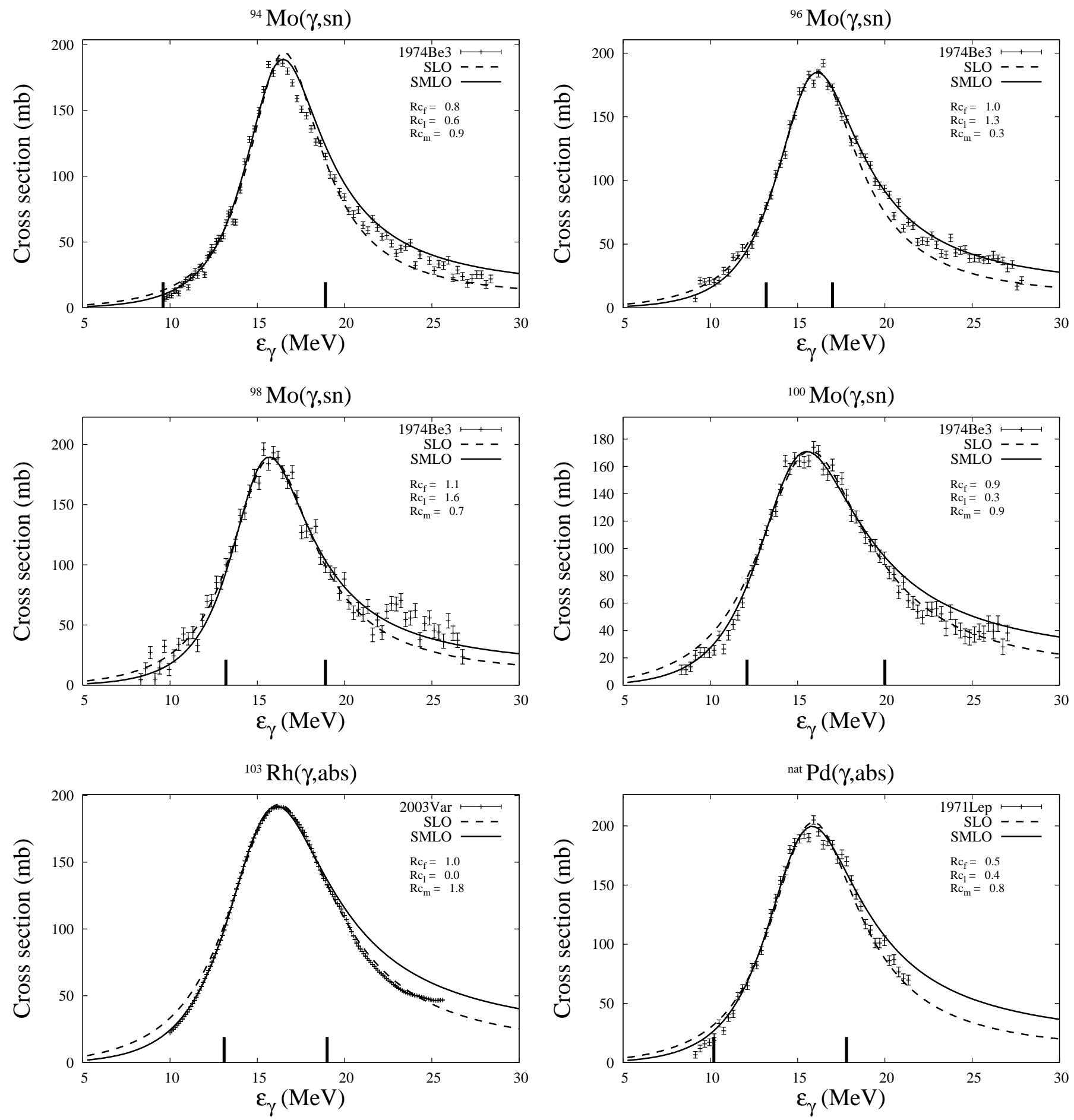

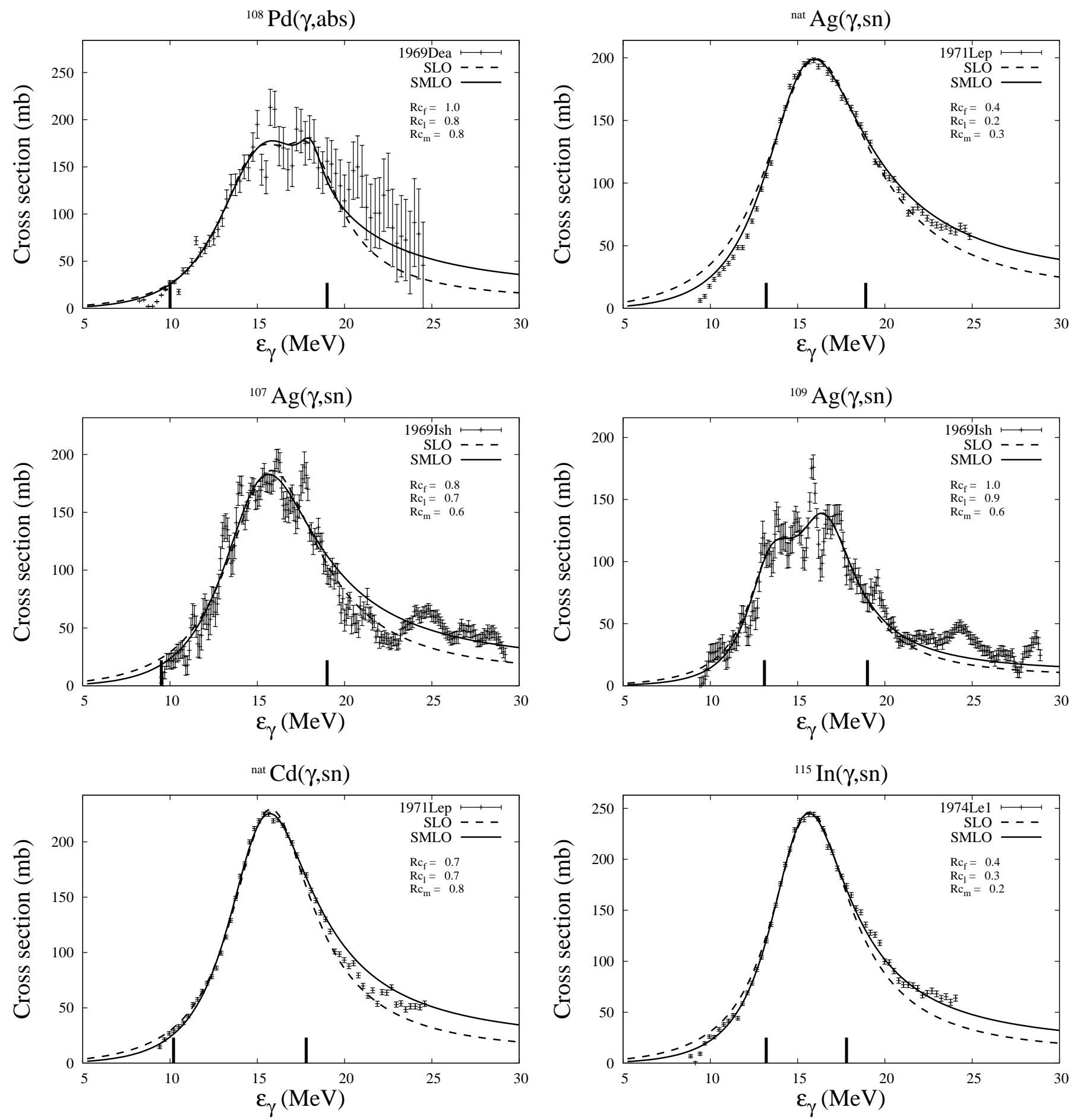

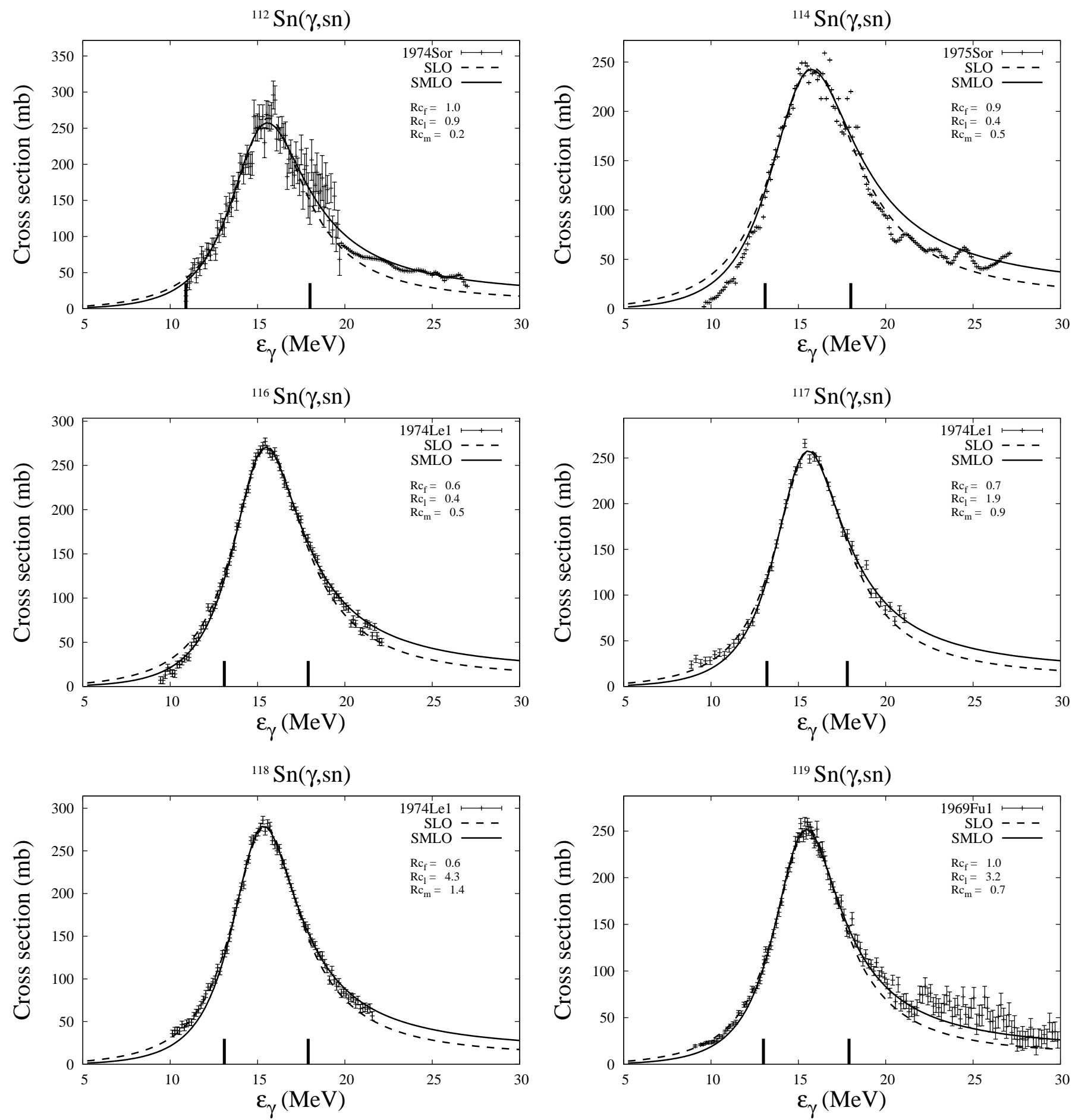

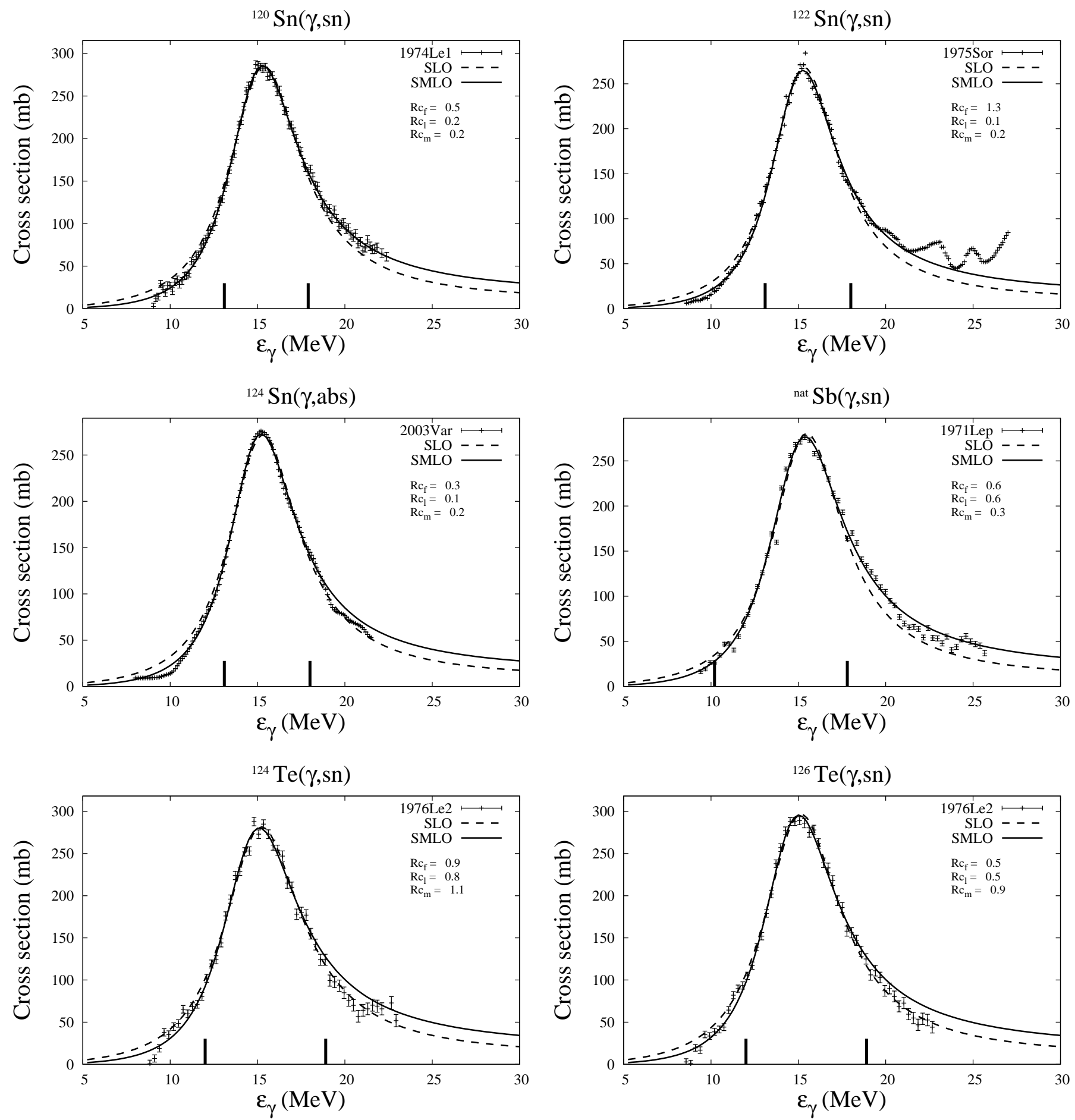

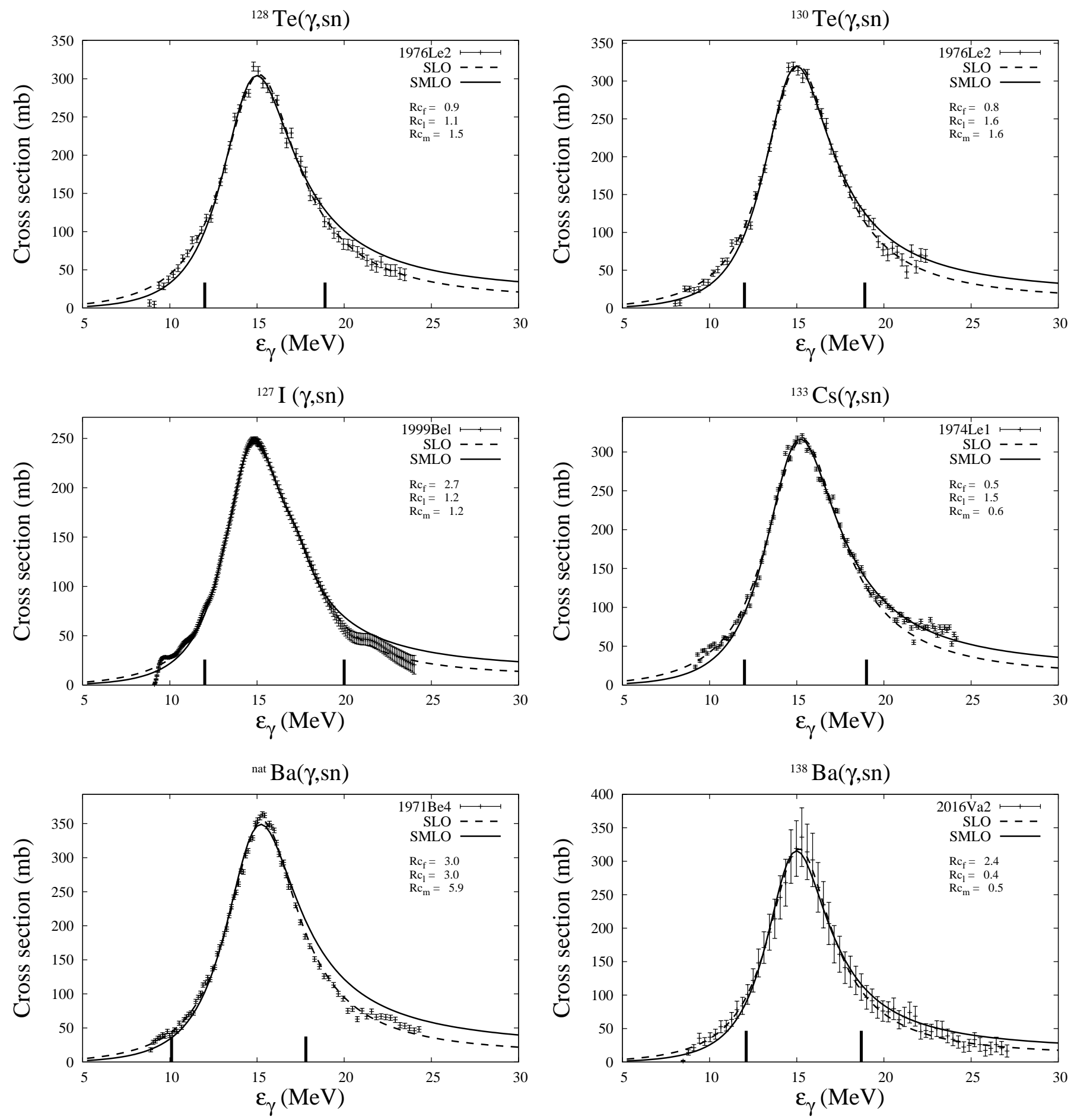

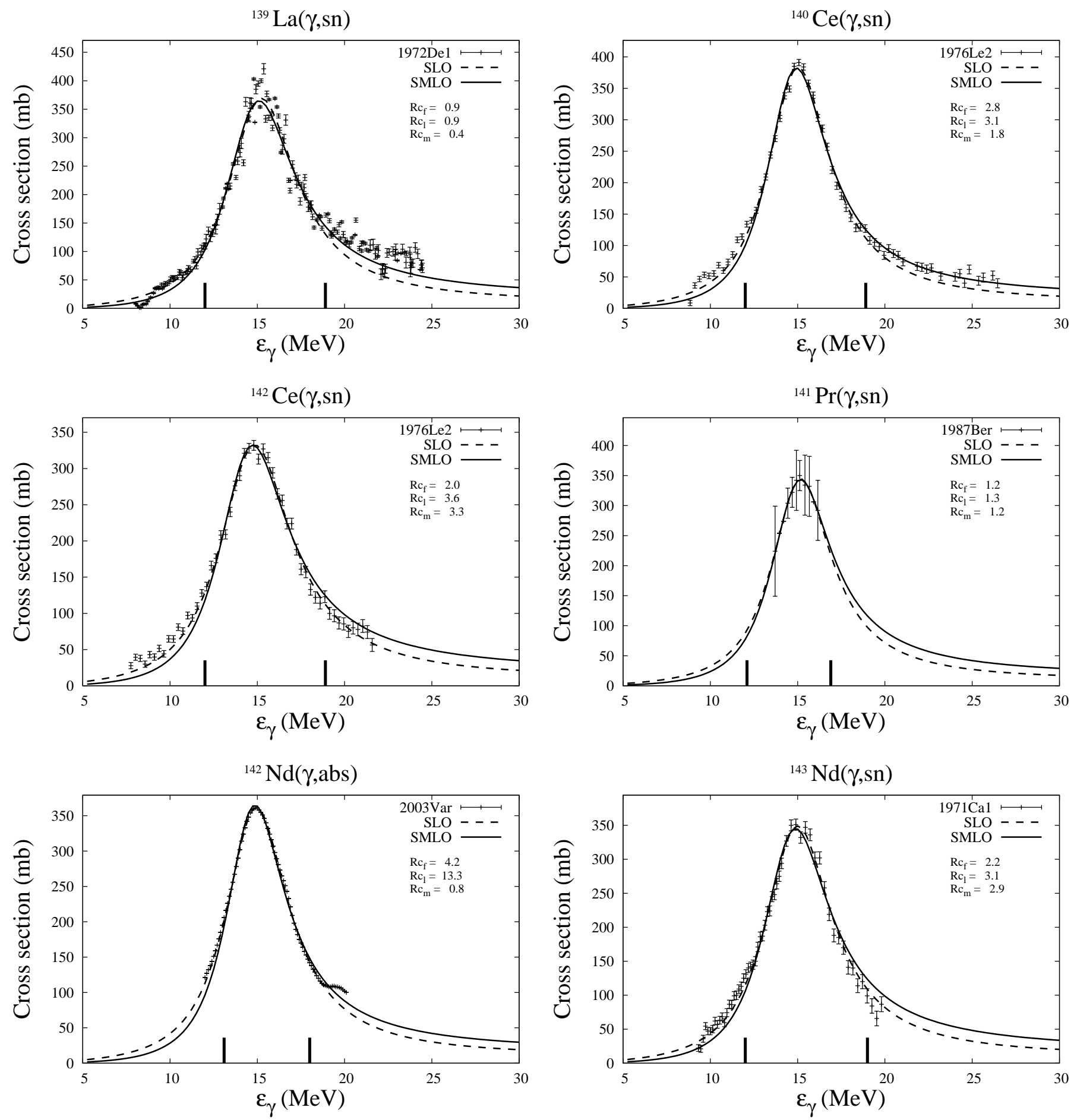

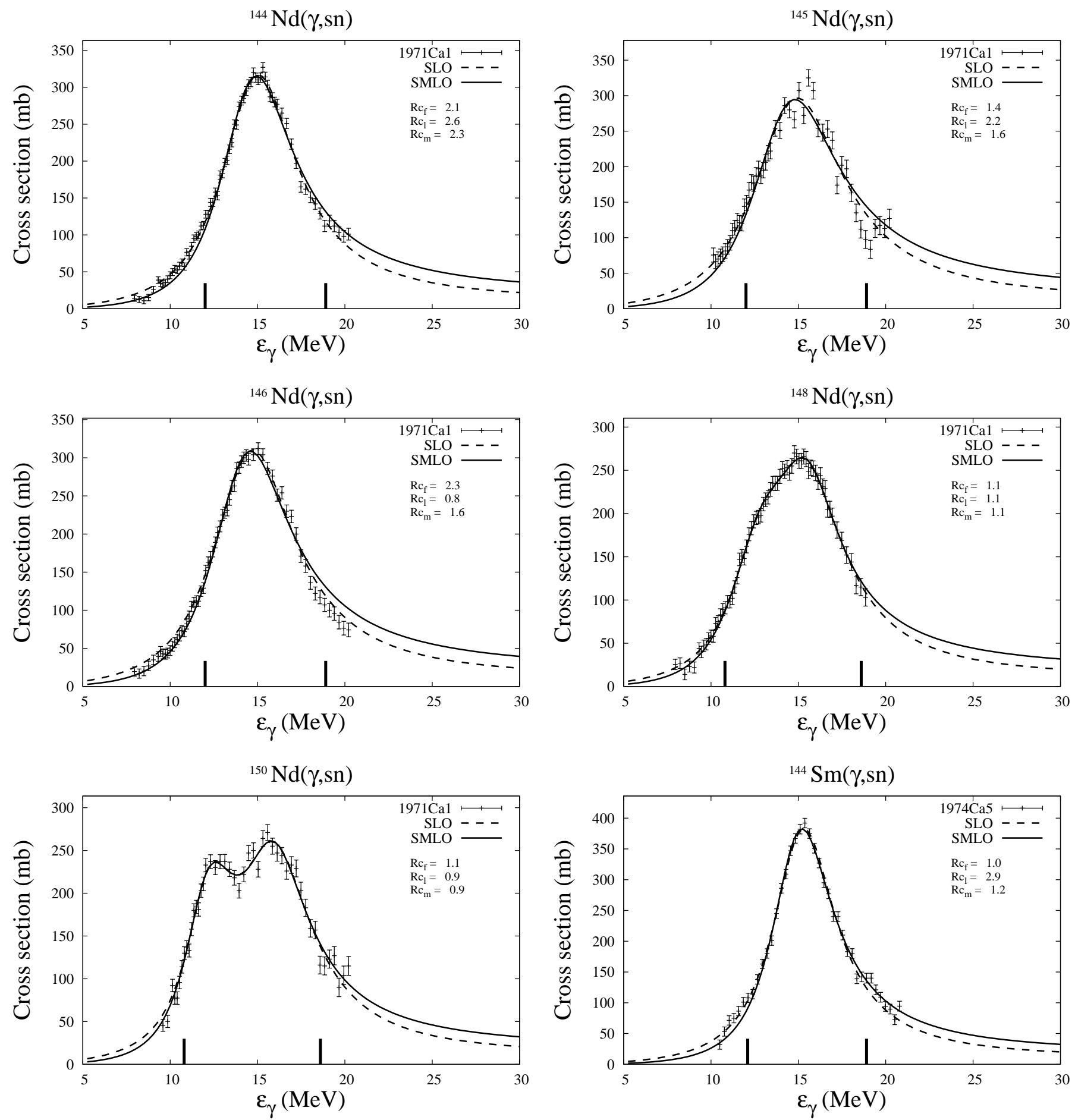

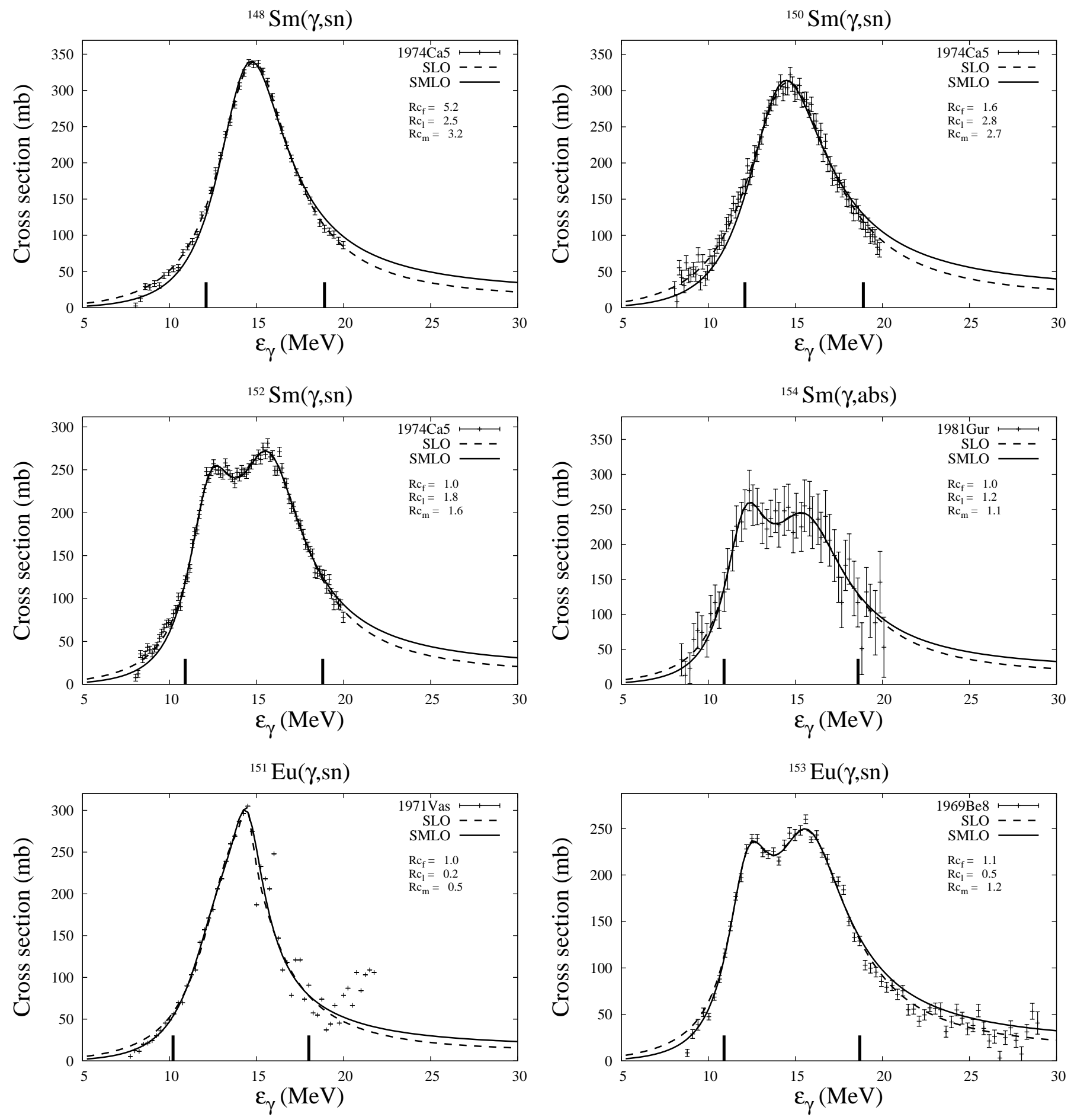

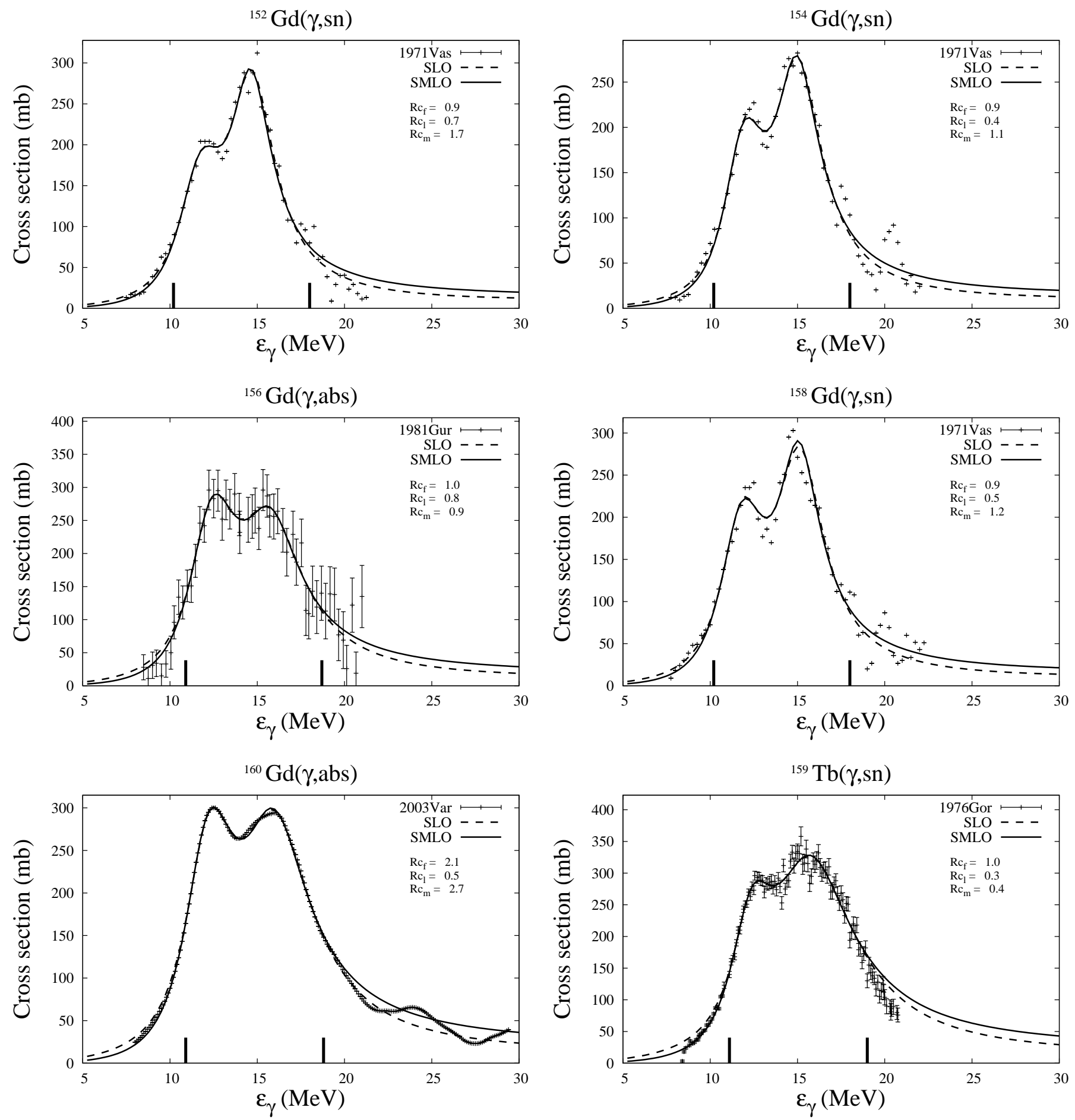

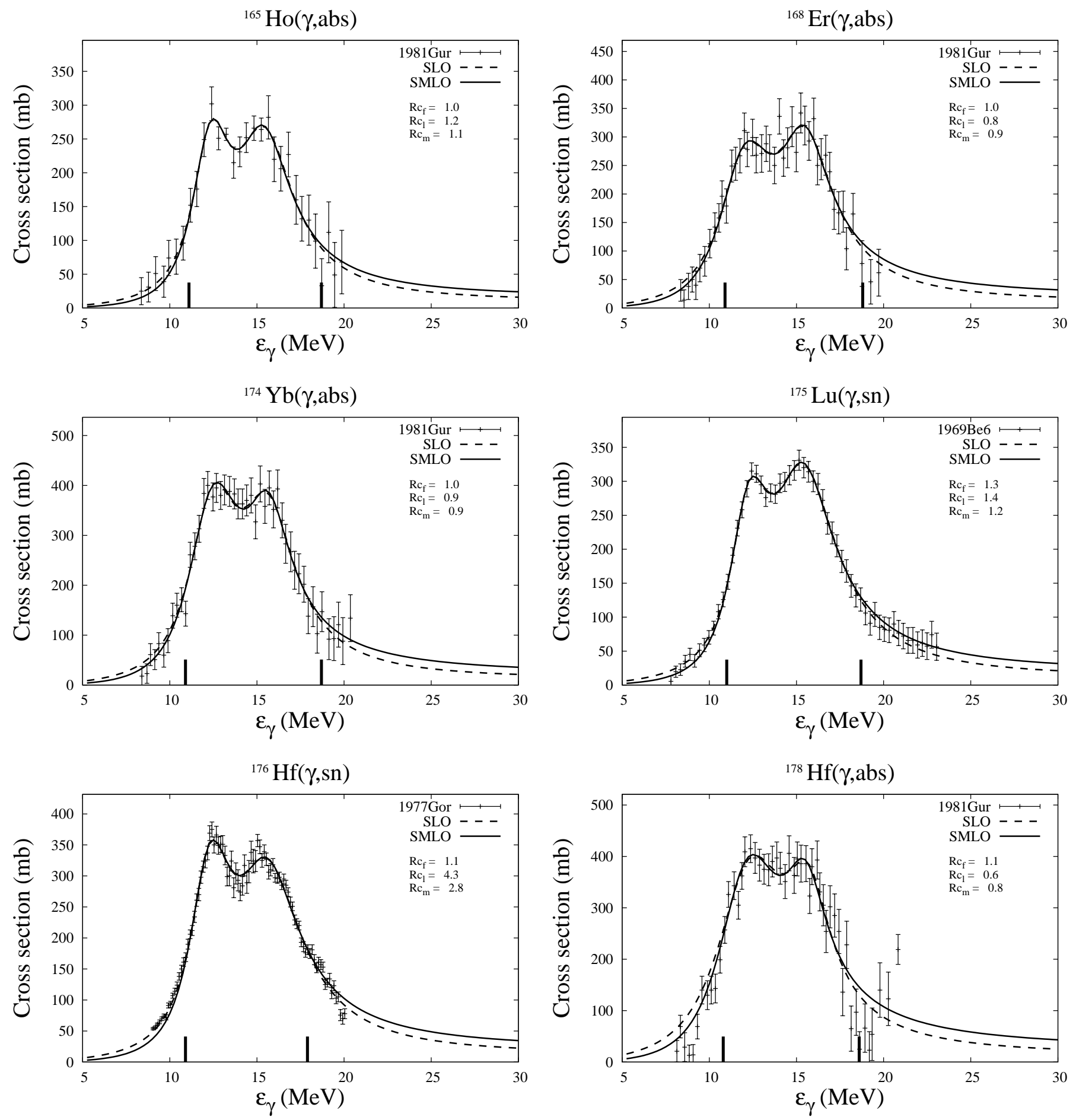

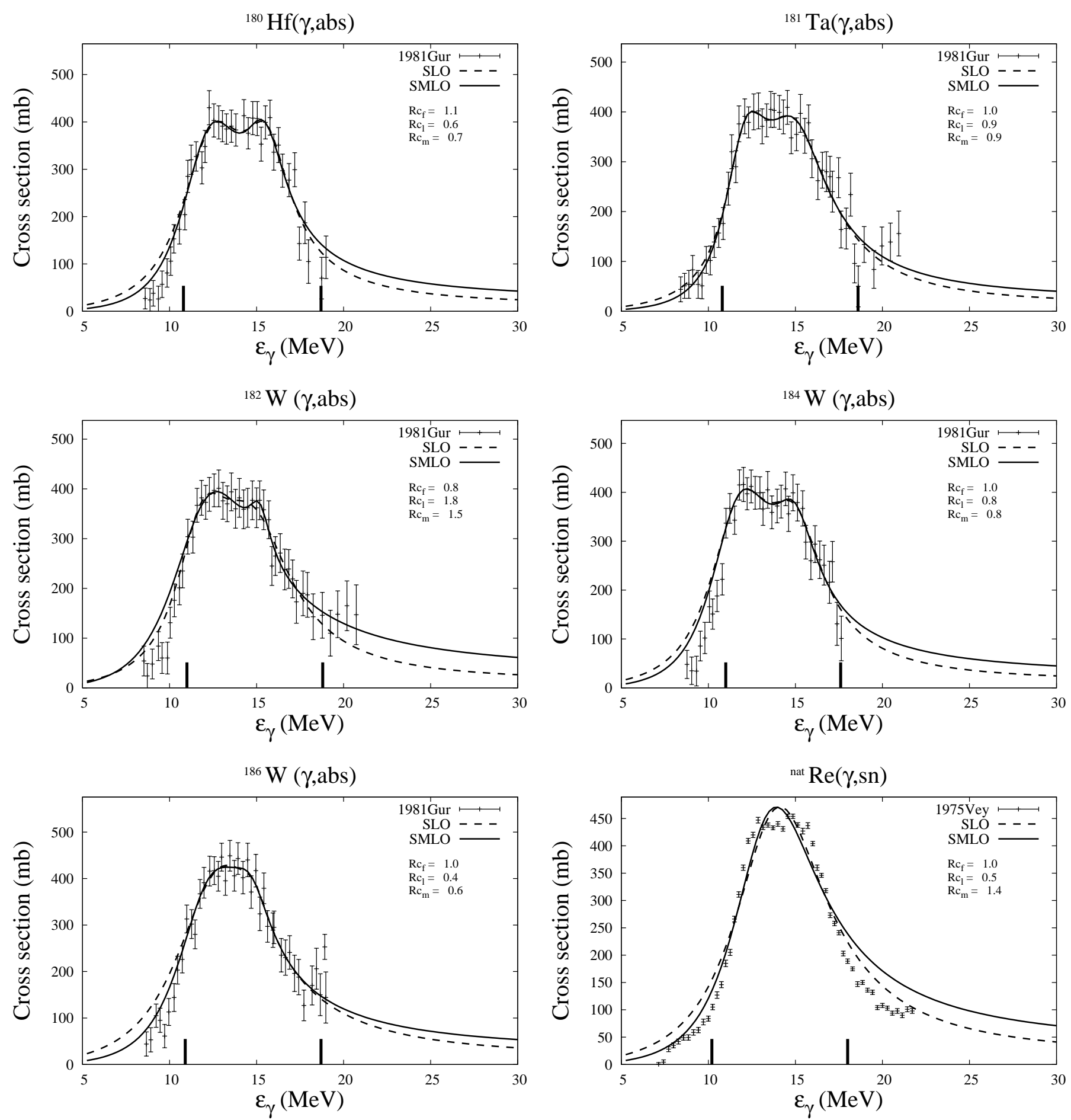

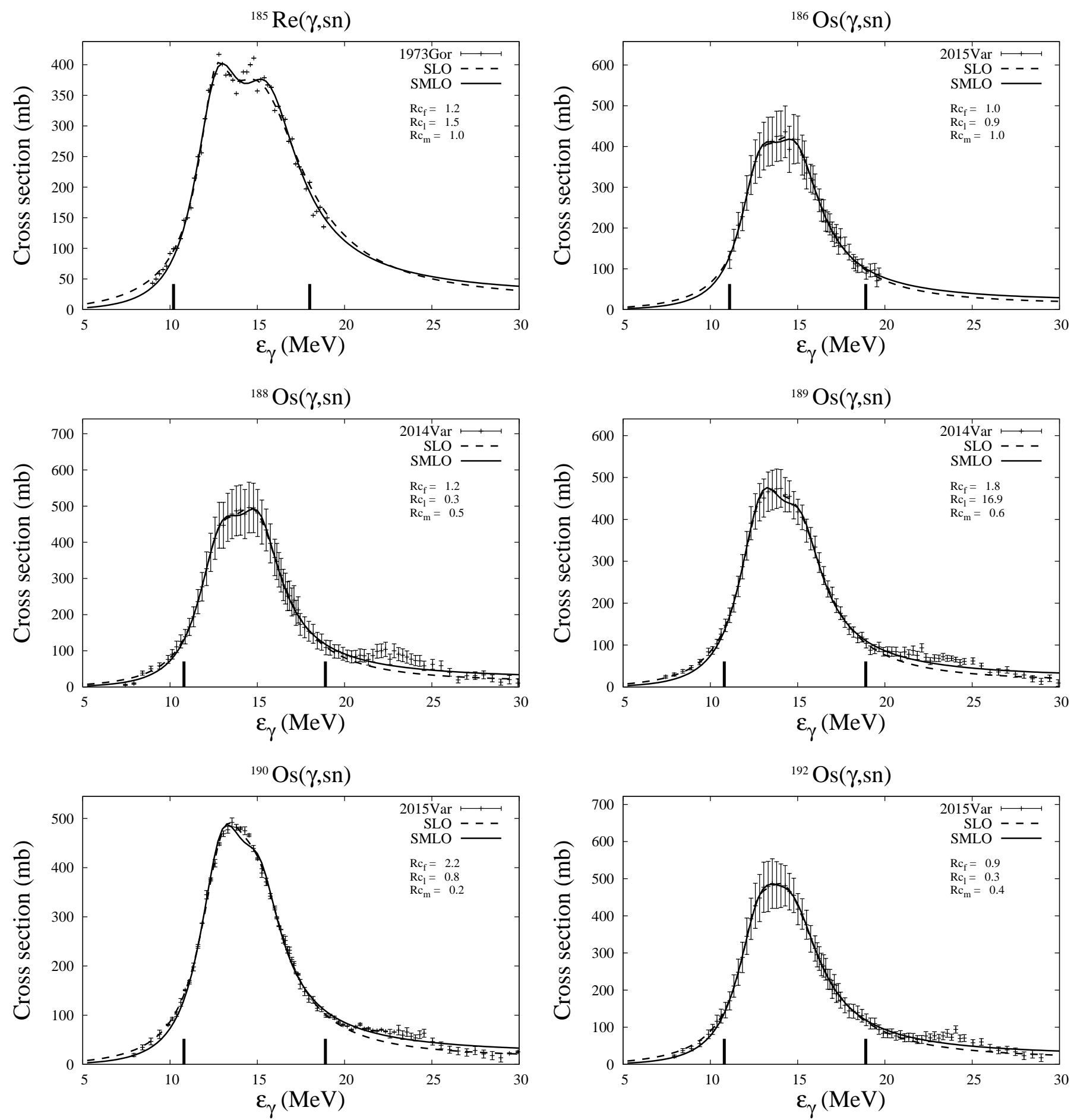

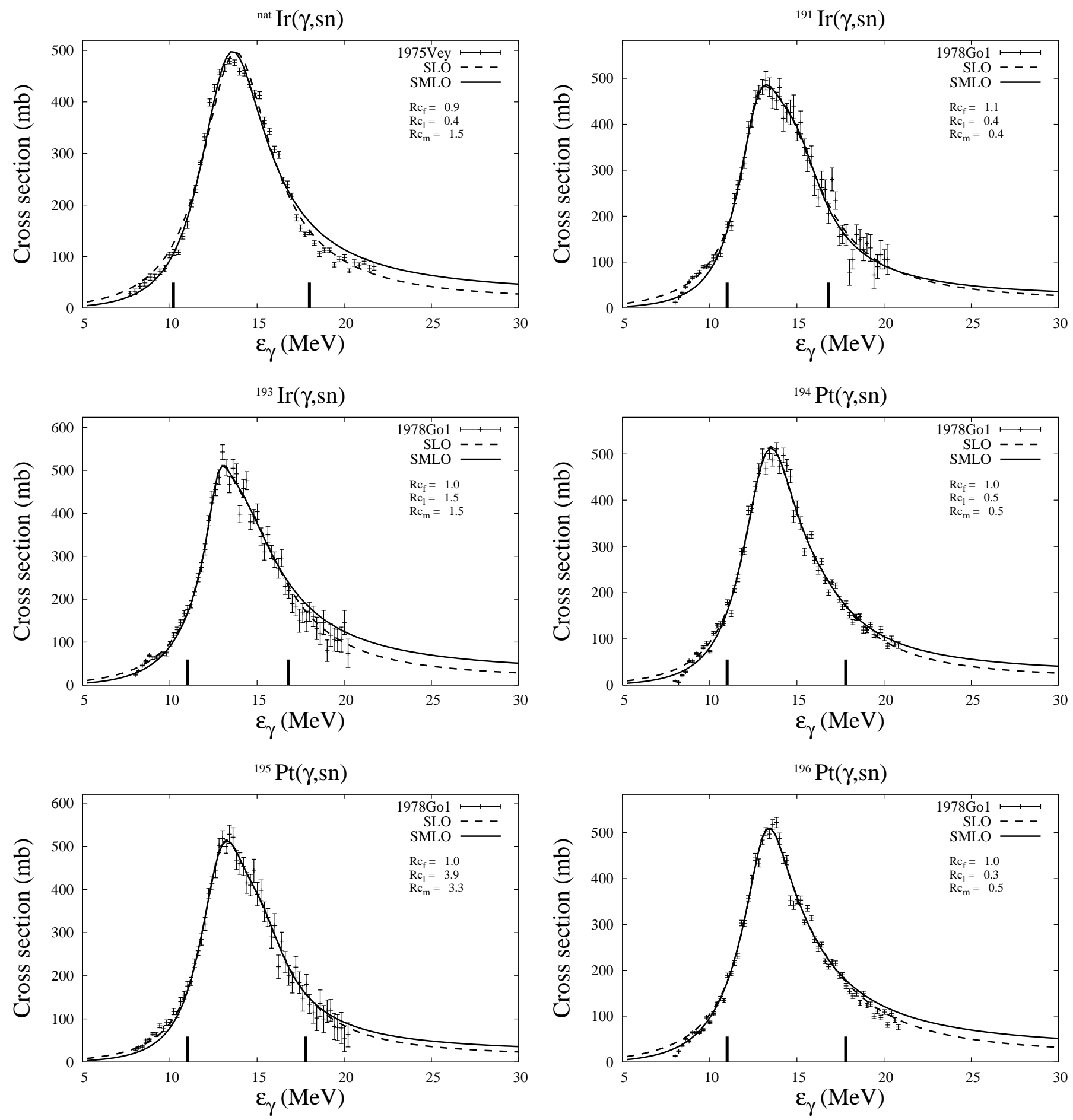

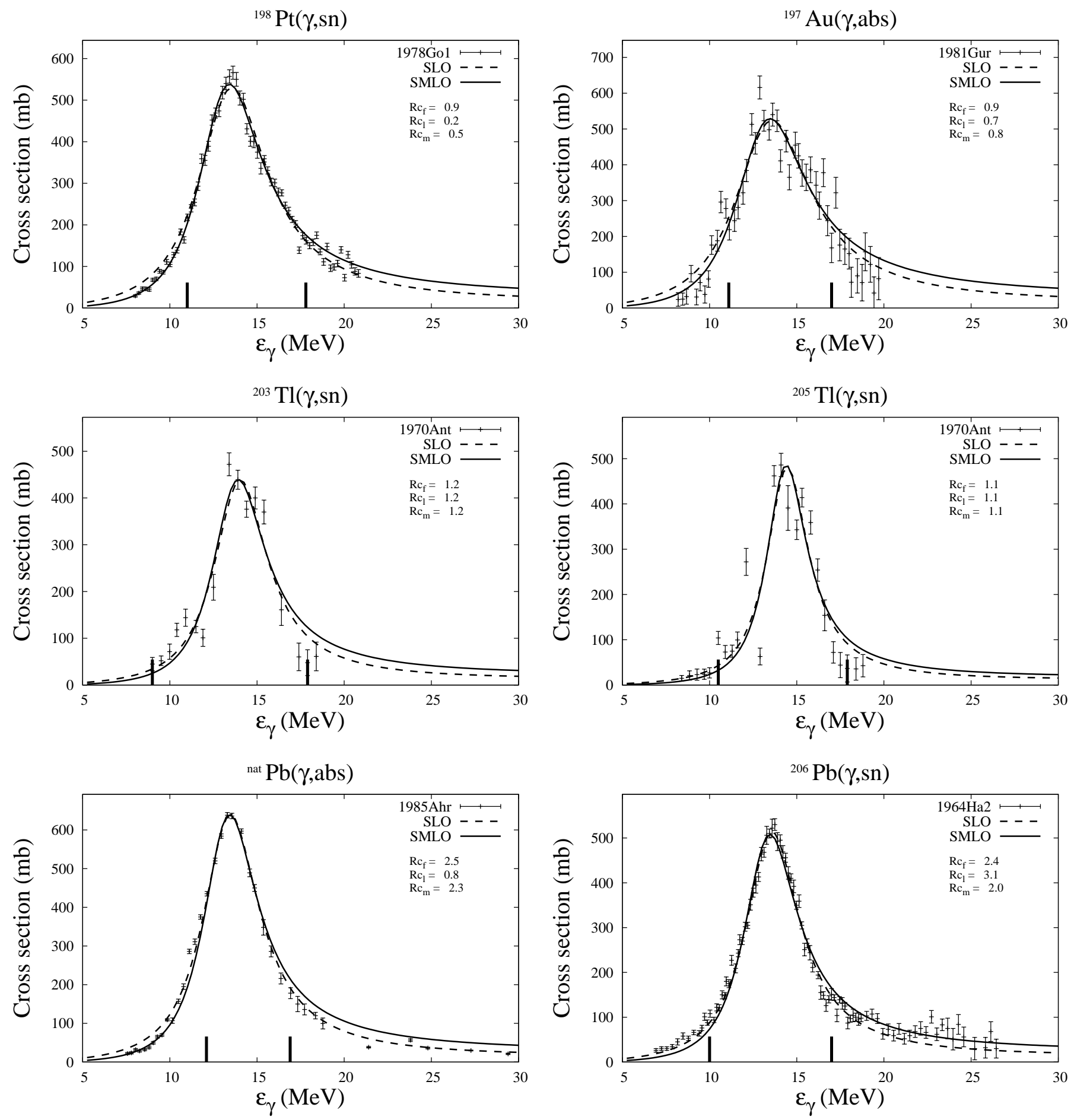

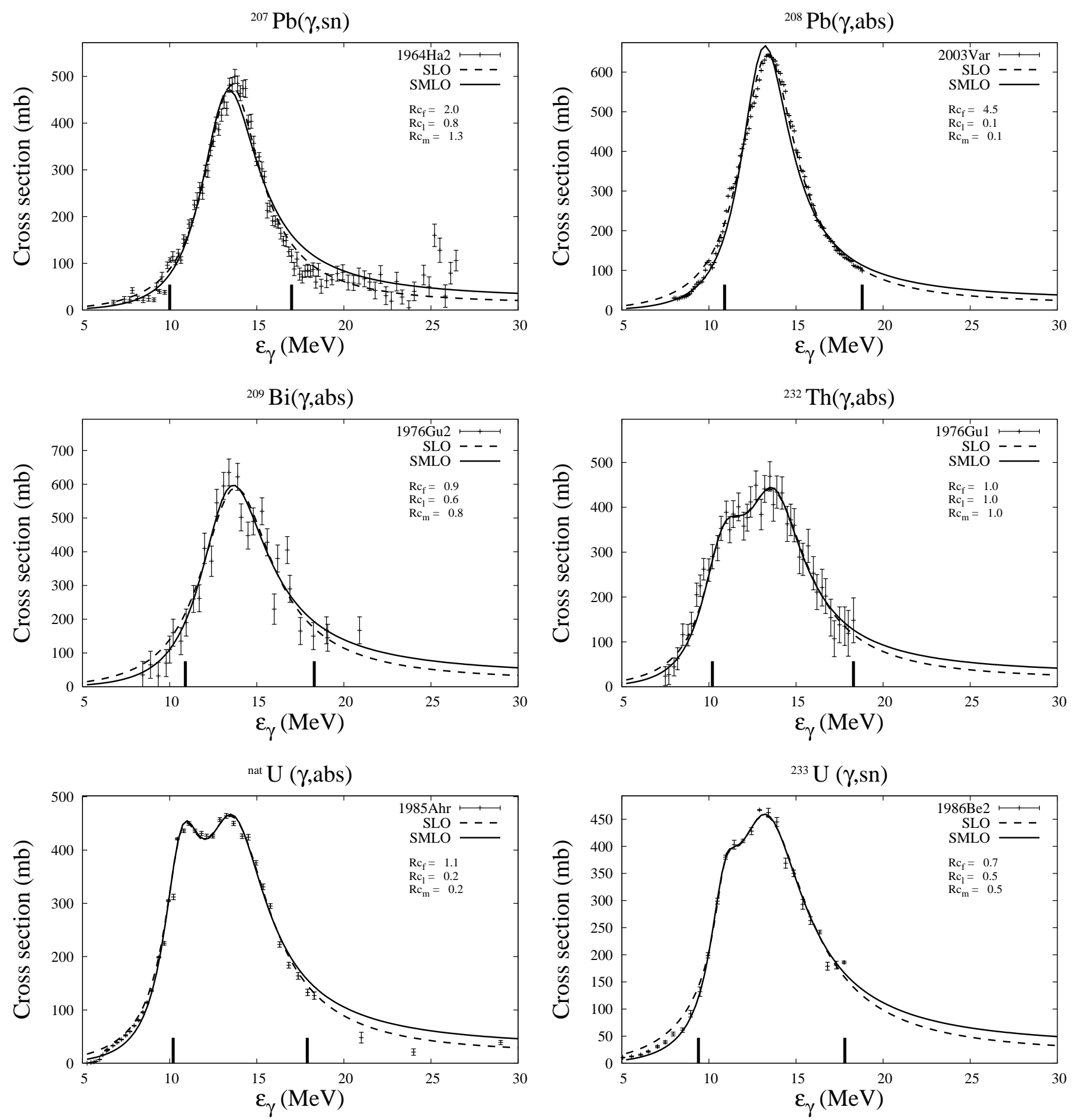

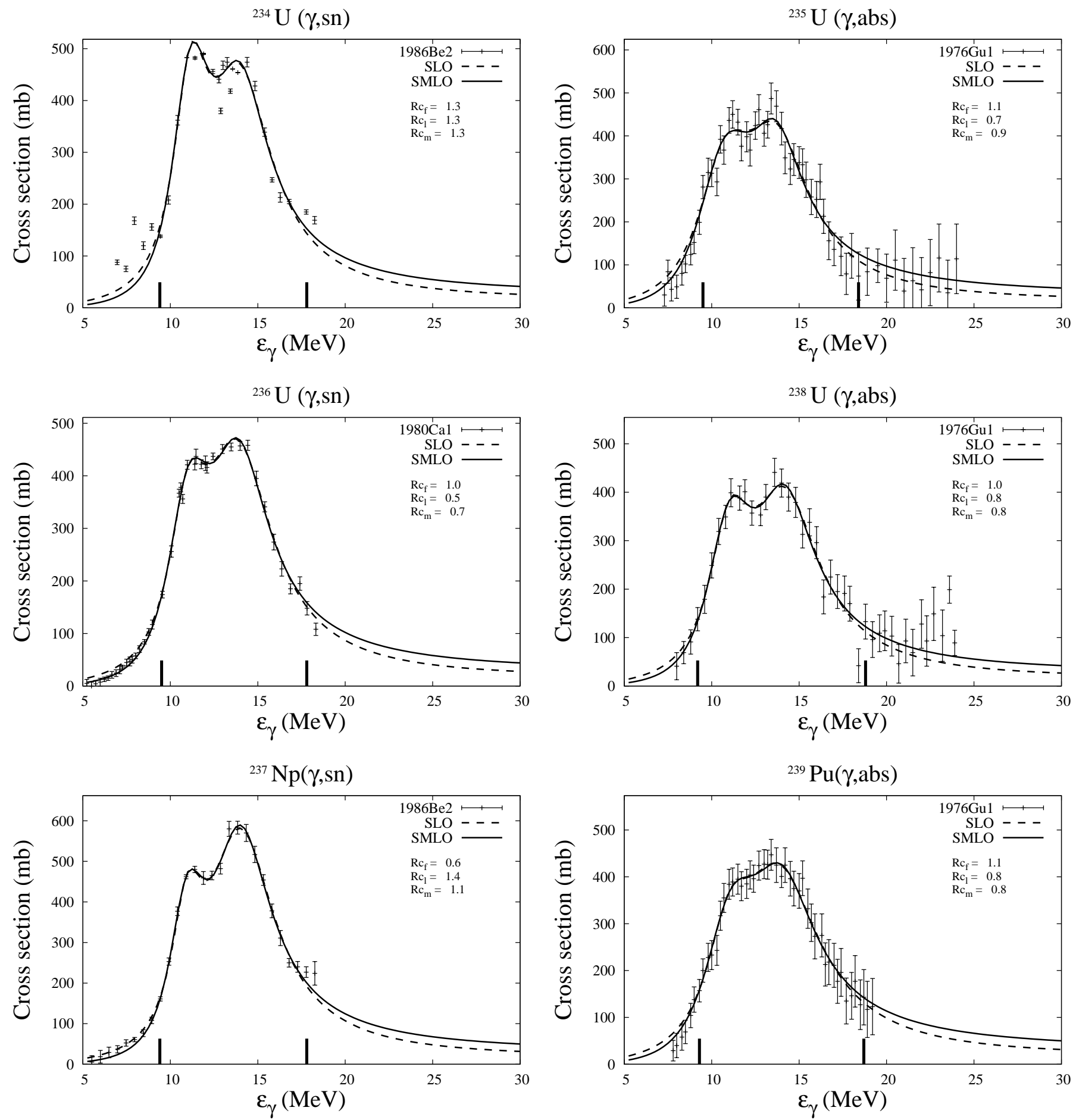